Eckart Bauer

Konzeptionelle

Grundfragen eines

Kinderleistungsausgleichs

im Rahmen einer

umlagefinanzierten

zwangsweisen

Rentenversicherung 


\section{Konzeptionelle Grundfragen eines Kinderleistungsausgleichs im Rahmen einer umlagefinanzierten zwangsweisen Rentenversicherung}

Die Arbeit untersucht die Bedeutung von Kindern für eine umlagefinanzierte Rentenversicherung. Nach einer Thematisierung grundsätzlicher Fragestellungen wird für verschiedene "Idealtypen“ von Umlageverfahren der jeweilige „materielle“ Wert von Kindern verdeutlicht. Anschließend erfolgt eine Simulation der distributiven Wirkungen einer Berücksichtigung „generativer Leistungen“. Die gewonnenen Erkenntnisse werden dann zu einer Beurteilung der Berücksichtigung der Kindererziehung im gegenwärtigen deutschen Rentenrecht herangezogen.

Eckart Bauer studierte von 1989 bis 1995 Volkswirtschaftslehre an der Universität zu Kiel. Von 1995 bis 2000 arbeitete er als wissenschaftlicher Mitarbeiter am Institut für Finanzwissenschaft der Universität der Bundeswehr Hamburg. Promotion 2000. 
Konzeptionelle Grundfragen

eines Kinderleistungsausgleichs im Rahmen

einer umlagefinanzierten zwangsweisen Rentenversicherung 


\section{SCHRIFTEN ZUR \\ WIRTSCHAFTSTHEORIE UND WIRTSCHAFTSPOLITIK}

Herausgegeben von

Rolf Hasse, Jörn Kruse, Wolf Schäfer, Thomas Straubhaar und Klaus W. Zimmermann

\section{Band 19}

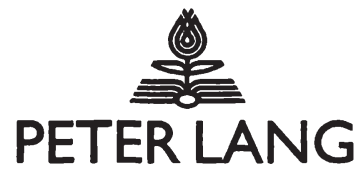

Frankfurt am Main - Berlin - Bern - Bruxelles - New York - Oxford - Wien 


\section{Eckart Bauer}

\section{Konzeptionelle Grundfragen eines Kinderleistungsausgleichs im Rahmen einer umlagefinanzierten zwangsweisen Rentenversicherung}

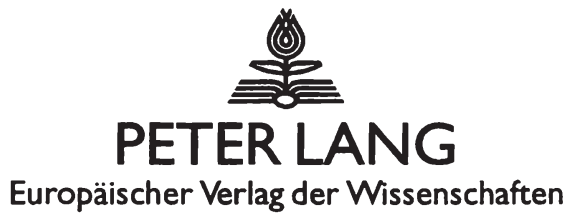


Die Deutsche Bibliothek - CIP-Einheitsaufnahme

Bauer, Eckart:

Konzeptionelle Grundfragen eines Kinderleistungsausgleichs im Rahmen einer umlagefinanzierten zwangsweisen

Rentenversicherung / Eckart Bauer. - Frankfurt am Main ; Berlin ;

Bern ; Bruxelles ; New York ; Oxford ; Wien : Lang, 2000

(Schriften zur Wirtschaftstheorie und Wirtschaftspolitik ;

Bd. 19)

Zugl.: Hamburg, Univ. der Bundeswehr, Diss., 2000

ISBN3-631-36800-3

Open Access: The online version of this publication is published on www.peterlang.com and www.econstor.eu under the international Creative Commons License CC-BY 4.0. Learn more on how you can use and share this work: http://creativecommons. org/licenses/by/4.0.

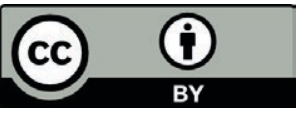

This book is available Open Access thanks to the kind support of ZBW - Leibniz-Informationszentrum Wirtschaft.

Gedruckt mit Unterstützung der Universität der Bundeswehr Hamburg.

Gedruckt auf alterungsbeständigem, säurefreiem Papier.

D705

ISSN 1433-1519

ISBN3-631-36800-3

ISBN 978-3-631-74995-1 (eBook)

(C) Peter Lang GmbH

Europäischer Verlag der Wissenschaften

Frankfurt am Main 2000

Alle Rechte vorbehalten.

Das Werk einschließlich aller seiner Teile ist urheberrechtlich geschützt. Jede Verwertung außerhalb der engen Grenzen des

Urheberrechtsgesetzes ist ohne Zustimmung des Verlages unzulässig und strafbar. Das gilt insbesondere für

Vervielfältigungen, Übersetzungen, Mikroverfilmungen und die Einspeicherung und Verarbeitung in elektronischen Systemen.

Printed in Germany 124567 


\section{Inhaltsübersicht}

1.

2.

2.1 .

2.2 .

2.3.

2.4 .

2.5.

2.6.

3.

3.1.

3.2.

3.3.

4.

4.1.

4.2 .

4.3 .

5.

5.1 .

5.2.

6.

6.1 .

6.2. Kann es eine politische Mehrheit für eine kinderfreundliche Reform der Rentenversicherung geben?.

7.

Einführung.

Normative und grundlegende institutionelle

Ausgestaltungselemente.

Zwangsweise vs. freiwillige Alterssicherung..................................23

Allgemeine Gründe für einen Kinderleistungsausgleich ................30

Institutionelle Möglichkeiten einer Berücksichtigung von

,Kindererziehungsleistungen'.

Charakteristika einer Rentenversicherung

Auswirkungen einer Rentenversicherung auf die Entwicklung

der Geburtenzahlen

Rentenversicherung im Kapitaldeckungsverfahren und

Kinderberücksichtigung

Kinderberücksichtigung bei einer im Umlageverfahren

organisierten Rentenversicherung

Grundlegende konzeptionelle Versionen einer

umlagefinanzierten Rentenversicherung...

Versionen mit Fixierung von Beiträgen oder Renten

(Versionen 1 und 2).

Versionen mit relativen Absicherungs- oder Belastungsvorgaben 78

Die distributiven Konsequenzen einer (partiellen)

Internalisierung der externen Effekte des Kinderhabens im

Rahmen einer umlagefinanzierten Zwangsrentenversicherung ... 127

Annahmen, Anforderungen und Ausgestaltungselemente.

Der Analyserahmen.

Verteilungsanalyse

Kinderberücksichtigung im Rahmen einer zwangsweisen umlagefinanzierten Rentenversicherung - ein Vorschlag und die deutsche Realität .

Der Vorschlag BORCHERTs zur Kinderberücksichtigung in der

Rentenversicherung.

Die gegenwärtige Regelung im deutschen Rentenrecht in kritischer Sicht

Schlussbetrachtung.

Wie wahrscheinlich sind Änderungen des generativen

Verhaltens bei verstärkter Kinderberücksichtigung im Rahmen der Rentenversicherung?

Anhänge

23

.39

.201 
7.1. Anhang 1: Formale Darstellung der Kinderberücksichtigung während der Beitragszeit bei einer Rentenversicherung nach Version 2

7.2. $\quad$ Anhang 2: Umformung von Version 1 zu Version 2 unter einfachen Bedingungen .................................................... 223

7.3. Anhang 3: Zur Berechnung der bedingten Erwartungswerte ...... 225

7.4. Anhang 4: Abschätzung der impliziten Kosten der Kinderberücksichtigung in Form einer beitragsfreien Anrechnung von Kindererziehungszeiten im Rahmen der GRV ............................ 227

7.5. Anhang 5: Relevante Entscheidungen des

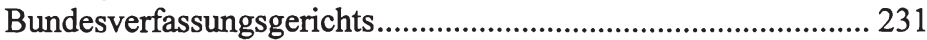

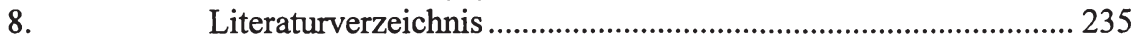




\section{Inhaltsverzeichnis}

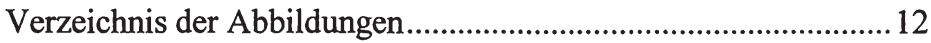

Verzeichnis der Tabellen.............................................................12

Verzeichnis der verwendeten Abkürzungen ..................................13

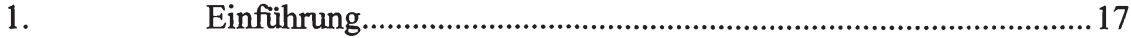

2. Normative und grundlegende institutionelle

2.1. Zwangsweise vs. freiwillige Alterssicherung...............................23

2.1.1. (Mögliche) Unterschiede in den distributiven Wirkungen freiwilliger und zwangsweiser Alterssicherungssysteme...............23

2.1.2. Rentenversicherung und, Altersarmut' ......................................27

2.2. Allgemeine Gründe für einen Kinderleistungsausgleich ...............30

2.2.1. Input- und outputorientierte Begründungsansätze .........................30

2.2.2. Exkurs: Zur Ökonomisierung.................................................... 33

2.3. Institutionelle Möglichkeiten einer Berücksichtigung von ,Kindererziehungsleistungen' ...............................................................

2.3.1. Kinderleistungsausgleich ausschließlich im Rahmen der

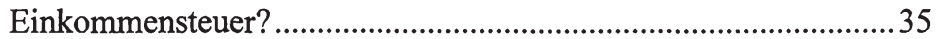

2.3.2. Beitragsäquivalenz als Maßstab ..............................................36

2.3.3. Ausgestaltung einer Kinderberücksichtigung bei Existenz

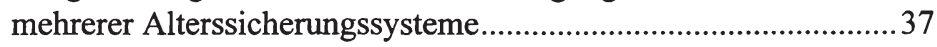

2.4. Charakteristika einer Rentenversicherung ……................................39

2.4.1. Die zwei Grundaufgaben einer Rentenversicherung.......................40

2.4.2. Kinderberücksichtigung in der Rentenversicherung - eine ,versicherungsfremde Leistung'?

2.5. Auswirkungen einer Rentenversicherung auf die Entwicklung

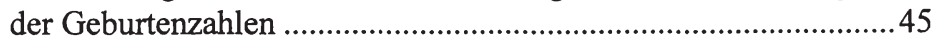

2.5.1. Existenzeffekte eines Rentenversicherungssystems.....................45

2.5.2. Ausgestaltungseffekte der Rentenversicherung ...............................46

2.5.3. Empirische Befunde zu den generativen Wirkungen von Rentenversicherungen

2.6. Rentenversicherung im Kapitaldeckungsverfahren und Kinderberücksichtigung ...

3. Kinderberücksichtigung bei einer im Umlageverfahren organisierten Rentenversicherung .................................................5

3.1. Grundlegende konzeptionelle Versionen einer umlagefinanzierten Rentenversicherung.

3.1.1. Die Grundgleichung des Umlageverfahrens einer

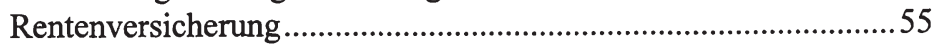

3.1.2. Vier Versionen eines Umlageverfahrens im Überblick ..................56

3.2. Versionen mit Fixierung von Beiträgen oder Renten 
(Versionen 1 und 2).....

3.2.1. Version 1 eines Umlageverfahrens unter statischen

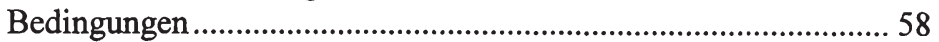

3.2.1.1. Vorbemerkungen ...................................................................... 58

3.2.1.2. Zur Bedeutung von Kindern für das durchschnittliche

Rentenniveau im Zeitablauf bei einer umlagefinanzierten

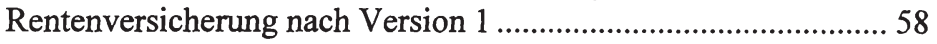

3.2.1.3. Möglichkeiten der Kinderberücksichtigung ................................. 61

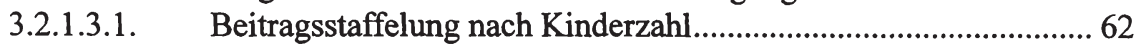

3.2.1.3.1.1. Eigenschaften einer Beitragsstaffelung nach Kinderzahl ............... 62

3.2.1.3.1.2. Formale Darstellung der Kinderberücksichtigung und einige

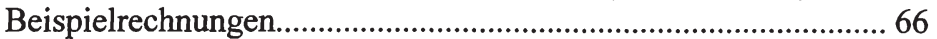

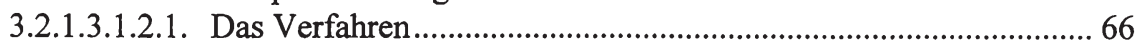

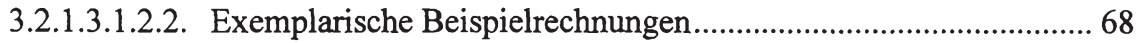

3.2.1.3.2. Rentenstaffelung nach Kinderzahl.................................................. 69

3.2.2. Version 2 eines Umlageverfahrens unter statischen Bedingungen 70

3.2.2.1. Beziehungen zwischen Version 1 und Version 2 .......................... 70

3.2.2.2. Kinderberücksichtigung in Version 2 ......................................... 71

3.2.2.3. Wirkungen von Änderungen in der Wachstumsrate der Stärke der Aktivgeneration in den Versionen 1 und 2............. 71

3.2.2.3.1. Darstellung und Vergleich der Belastungswirkungen ................... 72

3.2.2.3.2. Abwägung der Vorteilhaftigkeit einer Ausgestaltung nach

Version 1 und Version 2 im Falle einer Änderung der

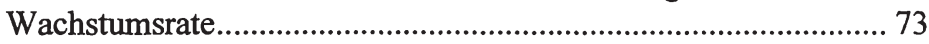

3.2.2.3.3. Der Aussagegehalt der Versionen 1 und 2 kritisch hinterfragt...... 75

3.2.2.3.4. Vergleich der beiden Ansätze zur Kinderberücksichtigung .......... 76

3.3. Versionen mit relativen Absicherungs- oder Belastungsvorgaben 78

3.3.1. Gründe für eine Analyse der Versionen 3 und 4 .......................... 78

3.3.1.1. Teilhabe der Rentner an Produktivitätsfortschritten........................ 78

3.3.1.2. Induzieren in einem Umlageverfahren Beitragszahlungen

Rentenansprüche? ......................................................................... 79

3.3.1.2.1. Betrachtung eines ,naturwüchsigen' Umlageverfahrens ................ 80

3.3.1.2.2. Betrachtung eines familienübergreifenden kollektiven

3.3.1.3. Abgabewiderstände' gegen Rentenversicherungsbeiträge? ......... 84

3.3.1.4. Probleme der ,Renditemessung ' für ein Umlageverfahren ........... 88

3.3.1.5. Besonderheiten der individuellen Rentensicherung durch

Beitragsleistungen bei einem Beitragssatz-Umlageverfahren ........ 91

3.3.1.5.1. Höhere Renten der Eltern infolge höherer Beitragszahlungen ihrer Kinder................................................................................. 92

3.3.1.5.2. Höhere eigene Renten infolge höherer eigener Beitragszahlungen93

3.3.1.5.2.1. Anreizwirkungen ............................................................................ 93

3.3.1.5.2.2. Exkurs: Unterschiede und Gemeinsamkeiten von Änderungen 
des Beitragssatzes und der Bemessungsgrundlage.

3.3.1.5.2.3. Exkurs: Ausweitung des Kreises der Versicherungspflichtigen als Lösung von Finanzproblemen der GRV?

3.3.2. Version 3 eines Umlageverfahrens in differenzierter Sicht ..........100

3.3.2.1. Darstellung einer umlagefinanzierten Rentenversicherung

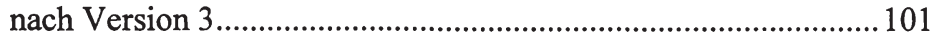

3.3.2.2. Das Kindeseinkommen ......................................................... 101

3.3.2.3. Zur Bedeutung und Berücksichtigung von Kindern bei einer umlagefinanzierten Rentenversicherung nach Version 3.............102

3.3.2.3.1. Externe Effekte von Kindern........................................................ 102

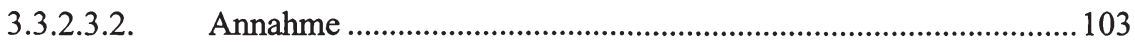

3.3.2.3.3. Substitution von eigenen Beitragszahlungen durch

Beitragszahlungen der Kinder.

3.3.2.3.4. ,Einkommen der Kinder' statt ,Kinderzahl' als Indikator ,elterlicher Leistungen' - Vergleich mit Version 1 ...................... 104

3.3.2.3.5. Exkurs: Zum Begriff , Kinderqualität' ........................................105

3.3.2.3.6. Probleme der Kinderberücksichtigung........................................... 107

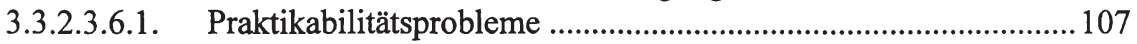

3.3.2.3.6.2. Kinderberücksichtigung erst in der Rentenphase? ...................... 108

3.3.2.3.6.3. ,Elternrenten' auch für Schwiegerkinder? ................................... 109

3.3.3. Version 4 eines Umlageverfahrens in differenzierter Sicht .........110

3.3.3.1. Mögliche Indikatoren des Lebensstandards .................................111

3.3.3.2. Determinanten des Beitragssatzes zur Rentenversicherung bei einkommensbezogener Absicherung des Lebensstandards...........118

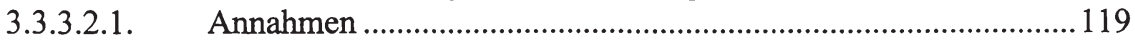

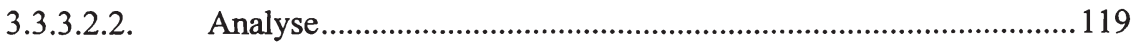

3.3.3.2.3. Erweiterungen ......................................................................... 123

3.3.3.3. Zur Bedeutung und Berücksichtigung von Kindern bei einer umlagefinanzierten Rentenversicherung nach Version 4............. 123

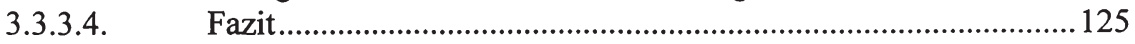

4. Die distributiven Konsequenzen einer (partiellen)

Internalisierung der externen Effekte des Kinderhabens im

Rahmen einer umlagefinanzierten Zwangsrentenversicherung ... 127

4.1. Annahmen, Anforderungen und Ausgestaltungselemente ............127

4.2. Der Analyserahmen...................................................................... 129

4.2.1. Die Aufteilung der Rente in einen kinder- und einen

beitragsbezogenen Teil im Konkreten..........................................130

4.2.2. Versicherung der Eltern gegen geringe Einkommen

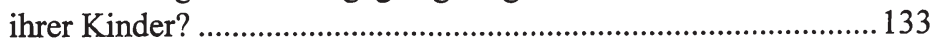

4.2.3. Zu weiteren Eigenschaften .......................................................... 134

4.3. Verteilungsanalyse ................................................................... 137

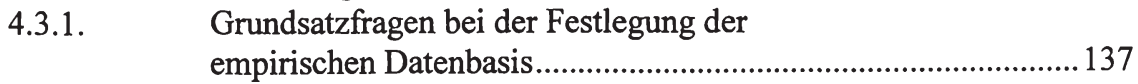


4.3.1.1. Basisdaten................................................................................. 137

4.3.1.2. Test auf Kalibrierung (des beitragsbezogenen Teils) ................... 138

4.3.1.3. Der ,Korrekturfaktor' und Probleme der Schätzung .................... 139

4.3.2. Simulation der rentenmäßigen Bedeutung von Kindern .............. 140

4.3.2.1. Die absolute Höhe der unterschiedlichen Rentenkomponenten .. 141

4.3.2.2. Substitution von Beitragszahlungen durch

,generative Leistungen ' ............................................................... 145

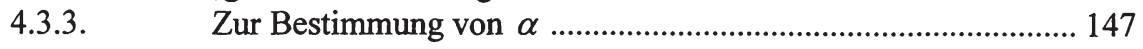

4.3.3.1. Hilfe durch Intuition? ............................................................ 147

4.3.3.2. Ansatzpunkte einer systemimmanenten Abschätzung.................. 147

4.3.3.2.1. Zum (richtigen) Diskontierungsfaktor in einem

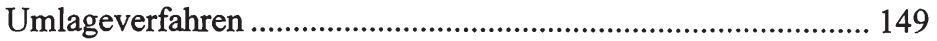

4.3.3.2.1.1. Diskontierung mit dem Kapitalmarktzinssatz ............................. 149

4.3.3.2.1.2. Diskontierung mit der Rate der , biologischen Verzinsung' ........ 150

4.3.3.2.1.3. Diskontierung mit der Zuwachsrate des rentenversicherungspflichtigen Einkommens.............................. 150

4.3.3.2.1.4. Diskontierung mit der Zuwachsrate des Beitragsaufkommens ... 151

4.3.3.2.1.5. Vergleich der Diskontfaktoren ..................................................... 151

4.3.3.2.2. Abschätzung von $\alpha$ für verschiedene Diskontierungsfaktoren.. 154

4.3.3.2.2.1. Abschätzung von $\alpha$ bei Diskontierung mit dem Kapitalmarktzinssatz ................................................................. 154

4.3.3.2.2.2. Abschätzung von $\alpha$ bei Diskontierung mit der Rate der ,biologischen Verzinsung'.......................................................... 155

4.3.3.2.2.3. Abschätzung von $\alpha$ bei Diskontierung mit der Zuwachsrate des rentenversicherungspflichtigen Einkommens ........................ 155

4.3.3.2.2.4. Abschätzung von $\alpha$ bei Diskontierung mit der Zuwachsrate des Beitragsaufkommens ............................................................ 155

4.3.3.2.2.5. Hinweis auf besondere Werte von $\alpha$..................................... 156

4.3.3.2.2.6. Abschätzung auf Basis der deutschen Rentenversicherung......... 157

4.3.3.3. Folgerung.................................................................................. 158

4.3.4. Mögliche Erweiterungen sowie Praktikabilitätsdimensionen .... 160

5. Kinderberücksichtigung im Rahmen einer zwangsweisen umlagefinanzierten Rentenversicherung - ein Vorschlag und die deutsche Realität....................................................................... 165

5.1. Der Vorschlag BORCHERTs zur Kinderberücksichtigung in der Rentenversicherung ................................................................... 165

5.1.1. Darstellung des Vorschlags .................................................... 165

5.1.2. Kritische Betrachtung des Vorschlags........................................... 166

5.1.3. Vergleich mit der vorliegenden Untersuchung............................ 170

5.2. Die gegenwärtige Regelung im deutschen Rentenrecht in kritischer Sicht.................................................................... 172

5.2.1. Die derzeitige Kinderberücksichtigung bei der Altersrente ........ 172

5.2.1.1. Darstellung der Kinderberücksichtigung bei der Altersrente ...... 172 
5.2.1.2. Bewertung der Ausgestaltung …..............................................175

5.2.2. Weitere Elemente der Kinderberücksichtigung im deutschen

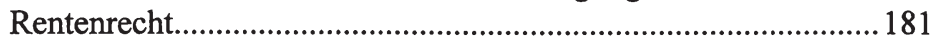

5.2.2.1. ,Aktive' Kinderberücksichtigung..................................................181

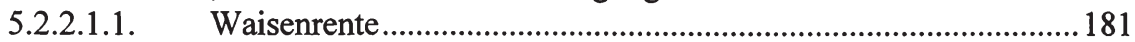

5.2.2.1.2. Witwen-, Witwer- und Erziehungsrente..........................................183

5.2.2.1.3. Sonderformen der Kinderberücksichtigung ..................................184

5.2.2.2. ,Passive' Kinderberücksichtigung.................................................185

5.2.2.3. Allgemeine Bewertung.............................................................187

5.2.3. Berücksichtigung verlängerter Rentenbezugsdauer ......................187

5.2.3.1. Änderung der Abgrenzung der Rentenberechtigten....................... 188

5.2.3.2. Rentenkürzung durch einen, demographischen Faktor ${ }^{6}$................190

5.2.3.3. Vergleich der unterschiedlichen Ansätze ....................................192

5.2.4. Exkurs: Nähe von ,Frauenförderung' zu ,Kinderförderung'? ......193

5.2.5. Zum Ausmaß der Kinderberücksichtigung

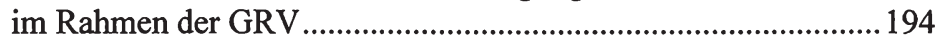

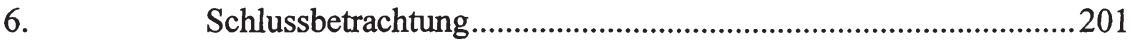

6.1. Wie wahrscheinlich sind Änderungen des generativen

Verhaltens bei verstärkter Kinderberücksichtigung im Rahmen der Rentenversicherung? ............................................201

6.2. Kann es eine politische Mehrheit für eine kinderfreundliche Reform der Rentenversicherung geben? ...................................216

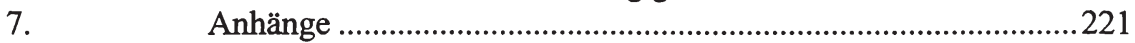

7.1. Anhang 1: Formale Darstellung der Kinderberücksichtigung während der Beitragszeit bei einer Rentenversicherung nach

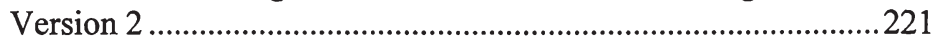

7.2. $\quad$ Anhang 2: Umformung von Version $1 \mathrm{zu}$ Version 2 unter einfachen Bedingungen.......................................................223

7.3. Anhang 3: Zur Berechnung der bedingten Erwartungswerte.......225

7.4. Anhang 4: Abschätzung der impliziten Kosten der Kinderberücksichtigung in Form einer beitragsfreien Anrechnung von Kindererziehungszeiten im Rahmen der GRV .......................227

7.5. Anhang 5: Relevante Entscheidungen des Bundesverfassungsgerichts .....................................................231

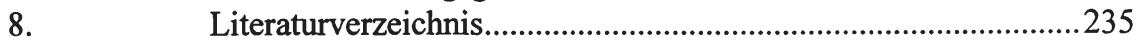




\section{Verzeichnis der Abbildungen}

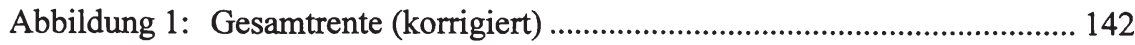

Abbildung 2: Kinderbezogene Rente (korrigiert) ............................................... 143

Abbildung 3: Rentenhöhe (korrigiert) in Abhängigkeit von der Beitrags position des Rentners und der kumulierten Beitragsposition

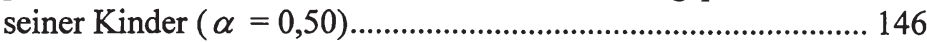

Abbildung 4: Grenzrate der Transformation zwischen Beitragsposition des Rentners und der kumulierten Beitragsposition seiner Kinder in Abhängigkeit von $\alpha$ bei Ausschöpfungskorrektur

\section{Verzeichnis der Tabellen}

Tabelle 1: Rentenwerte eines Rentners mit durchschnittlicher Beitragsposition in Abhängigkeit von $\alpha$..................................... 144

Tabelle 2: Abschätzung des ,richtigen' Grades der Beitragsorientierung für verschiedene Diskontierungsmethoden

Tabelle 3: Berechnungen Gallons zu den Rentenwirkungen des BORCHERT-Vorschlags

Tabelle 4: Auswirkungen der Anerkennung der Kindererziehung im Rentenrecht in den alten Bundesländern seit 1986

Tabelle 5: Berechnung der Wahrscheinlichkeiten möglicher ,Überlebenskombinationen' der Elternteile

Tabelle 6: Abschätzungen zum, Wert' der kinderbezogenen Rentenzusagen nach dem gegenwärtigen deutschen Rentenrecht

Tabelle 7: Durch Beitragszahlungen in den Jahren 1992 bis 1996 erworbene jährliche Rentenansprüche 


\section{Verzeichnis der verwendeten Abkürzungen}
Allgemein
BSP Bruttosozialprodukt
BVerfGE Entscheidungen des Bundesverfassungsgerichts
BZ Beitragszahlung(en)
CDU Christlich Demokratische Union Deutschlands
CSU Christlich-Soziale Union in Bayern
DGB Deutscher Gewerkschaftsbund
DM Deutsche Mark
EStG Einkommensteuergesetz
FAZ
FDP
GE
GKV
GPV
GRV
Frankfurter Allgemeine Zeitung für Deutschland, Frankfurt / Main
Kap.
Freie Demokratische Partei
KDV Kapitaldeckungsverfahren
KF Korrekturfaktor
Mrd. Milliarden
MuSchG Mutterschutzgesetz
RV Rentenversicherung
SGB Sozialgesetzbuch
SPD Sozialdemokratische Partei Deutschlands
Stat. Statistisches
Tab. Tabelle
UV Umlageverfahren
v.H. von Hundert
VDR Verband Deutscher Rentenversicherungsträger
Vol. Volume

\section{Formal}

$B_{t} \quad$ Beitragsaufkommen der Rentenversicherung in Periode $t$

$B R_{t}^{j} \quad$ beitragsbezogene Rente des Rentners $j$ in der Periode $t$

$\operatorname{COV}(X, Y)$ Kovarianz von $X$ und $Y$

$D_{t, t+1} \quad$ Diskontierungsfaktor zur Berechnung des Barwerts einer Zahlung in der Periode $t+1$ in der Periode $t$ 
$E[X] \quad$ Erwartungswert von $X$

$G R_{t}^{j} \quad$ Gesamtrente des Rentners $j$ in der Periode $t$

$\operatorname{GRi}(\alpha) \quad$ Gesamtrente bei einer kumulierten Beitragsposition der Kinder von $i$ und einem Anteil ,beitragsbezogener Renten' an der Rentensumme von $\alpha \cdot 100 \%$

$\widetilde{K} \widetilde{R}_{t}^{j} \quad$ unkorrigierte ,kinderbezogene' Rente des Rentners $j$ in der Periode $t$

$K R_{t}^{j} \quad$ korrigierte ,kinderbezogene' Rente des Rentners $j$ in der Periode $t$

$K R i(\alpha) \quad$ kinderbezogene Rente bei einer kumulierten Beitragsposition der Kinder von $i$ und einem Anteil ,beitragsbezogener Renten' an der Rentensumme von $\alpha \cdot 100 \%$

$N_{t} \quad$ Zahl der Beitragszahler Periode $t$

$N_{t-1} \quad$ Zahl der Rentner in Periode $t$

$N_{t, n} \quad$ Zahl der Beitragszahler in Periode $t$ mit $n\left(n \in N^{0}\right)$ Kindern

$N^{0} \quad$ Menge der natürlichen Zahlen und 0

$U \quad$ Nutzen

$\operatorname{VAR}(X) \quad$ Varianz von $X$

$W_{t} \quad$ Produktionswert in der Periode $t$

$X, Y \quad$ Zufallsvariablen

$b$

$b^{n}$

$b_{t}^{n}$

$b_{t}$

$b_{t}^{i}$

$g_{t}$

$i_{t, t+1}$

$p_{i}$

$r$

14 intertemporal konstanter Rentenversicherungsbeitrag

Rentenversicherungsbeitrag eines Beitragszahlers mit $n\left(n \in N^{0}\right)$ Kindern

Rentenversicherungsbeitrag eines Beitragszahlers mit $n\left(n \in N^{0}\right)$ Kindern in Periode $t$

durchschnittlicher Rentenversicherungsbeitrag eines Beitragszahlers in Periode $t$

Rentenversicherungsbeitrag des Beitragszahlers $i$ in Periode $t$

Wachstumsrate des Umfangs der Aktivgeneration von Periode $t-1$

bis Periode $t\left(\equiv \frac{N_{t-1}}{N_{t}}-1\right)$

Kapitalmarktzinssatz für eine Geldanlage von Periode $t$ bis Periode $t+1$

Preis einer Einheit des Gutes $i$

Rentenzahlung an einen Rentner 
$r_{t}^{j}$

$r_{t}$

$r_{t}$

$t$

$x_{i}$

$y_{t}^{r v, i}$

$y^{r, i}$

$y_{t}$

$y_{t}$

$\alpha$

$\beta$

$\phi$

$\widetilde{\phi}$

$\sigma$

$\mu_{X} \quad$ Mittelwert von $X$

Rentenzahlung an Rentner $j$ in Periode $t$

Rentenzahlung an Rentner $\imath$

durchschnittliche Rentenzahlung an einen Rentner in Periode $t$

Zeitindex

Menge des Gutes $X_{i}$

rentenversicherungspflichtiges Einkommen des Beitragszahlers $i$ in Periode $t$

rentenversicherungspflichtiges Einkommen der Person $i$

durchschnittliches Einkommen eines Beitragszahlers Periode $t$

durchschnittliches rentenversicherungspflichtiges Einkommen eines Beitragszahlers in Periode $t$

Anteil der Rentensumme, der ,beitragsbezogen“ aufgeteilt wird Beitragssatz zur Rentenversicherung

Verhältnis der Durchschnittsrente eines Rentners zum durchschnittlichen Bruttoarbeitseinkommen eines Beitragszahlers (,Bruttoabsicherungssatz')

$\widetilde{\phi} \quad$ Verhältnis der Durchschnittsrente eines Rentners zum durchschnittlichen Nettoarbeitseinkommen eines Beitragszahlers (,Nettoabsicherungssatz')

Grenzrate der Transformation 
Eckart Bauer - 978-3-631-74995-1

Downloaded from PubFactory at 01/11/2019 09:28:58AM

via free access 


\section{Einführung}

Die Zahl der Kinder pro Mitglied einer Generation liegt in Deutschland seit Mitte der 20'er Jahre dieses Jahrhunderts unterhalb des Wertes, bei dem eine nachfolgende Generation vergleichbarer Kopfzahl in das fortpflanzungsfähige Alter kommt. Eine solche nicht-bestandserhaltende Nettoreproduktionsrate existiert nicht nur in Deutschland, sondern in fast allen westeuropäischen Staaten sowie Japan. Zwischen den einzelnen Staaten gibt es hierbei allerdings erhebliche Unterschiede im Ausmaß der Unterschreitung der bestandserhaltenden Geburtenzahl. ${ }^{1}$ Die gesamtdeutsche Nettoreproduktionsrate betrug im Jahre 1996 ca. 0,629 (für Westdeutschland lag sie bei 0,667 , für Ostdeutschland bei 0,454$),{ }^{2}$ d.h. die Nachfolgegeneration wird beim Erreichen des fortpflanzungsfähigen Alters - so sich im generativen Verhalten kurzfristig keine deutlichen Änderungen ergeben, was unterstellt werden kann - nur einen Umfang von ca. 63 v.H. ihrer Elterngeneration haben.

Dass die Bevölkerung Deutschlands Geburtenzahlen realisiert, die eine (deutliche) Abnahme des Generationenumfangs zur Folge haben, ist in historischer Betrachtung bemerkenswert. Anders als in früheren Zeiten hat die geringe Zahl an (das Erwachsenenalter erreichenden) Kindern pro Mitglied der fortpflanzungsfähigen Generation ihre Ursache nicht in Epidemien, Katastrophen, Kriegen etc., sondern primär in einer Abnahme der durchschnittlichen Zahl der Kinder pro Erwachsenem. Dieser Rückgang ist derart ausgeprägt, dass er die Steigerung der, Überlebenswahrscheinlichkeit' Lebendgeborener (infolge verbesserter medizinischer und hygienischer Lebensbedingungen) mehr als kompensiert.

1 Vgl. hierzu DORBRITZ (1998); vgl. auch SCHWARZ (1998).

2 Vgl. Statistisches Bundesamt (1998), Fachserie 1, Reihe 1, S. 99, Tab. 9. Werden nur ,deutsche Staatsangehörige' betrachtet, so liegt der aktuelle Wert für Gesamtdeutschland bei 0,610, für das frühere Bundesgebiet bei 0,647 und fur das Beitrittsgebiet bei 0,453 (vgl. ebd., S. 99, Tab. 9). Zu den Werten für die neuen Bundesländer ist anzumerken, dass infolge des geringen Alters der Frauen bei der Geburt der Kinder in der ehemaligen DDR ein gewisser ,Verschiebeeffekt' (Hinauszögerung der Geburt auf einen späteren Zeitpunkt, ohne dass dabei die am Ende der fruchtbaren Phase realisierte Kinderzahl verändert wird) potenziell bedeutsam erscheint, und somit eine Annäherung der ostdeutschen Zahlen an die westdeutschen als möglich gelten kann. Ein Indiz hierfür mag die erhebliche Volatilităt der Nettoreproduktionsrate für die neuen Bundesländer sein: Von 1990 bis 1991 sank der Wert von 0,728 auf 0,472, erreichte 1993/94 einen Tiefpunkt mit 0,370, um von 1995 auf 1996 von 0,401 auf 0,454 zu steigen (Zur Auswirkung von ,Timing-Änderungen' vgl. DINKEL (1984b), S. 44 - 49.). Ebenso mag die Verbesserung der wirtschaftlichen Situation in den neuen Bundesländern - zusammen mit einem dadurch induzierten Rückgang der Verunsicherung der Bevölkerung - zu einer Steigerung der Nettoreproduktionsrate fuhren. 
Eine Abschwächung des Bevölkerungswachstums bis hin zu einer Verringerung der Generationenstärke hat für viele Teile des Wirtschaftslebens nicht unerhebliche Folgen - von möglicherweise negativen Auswirkungen der ,Alterung' einer Gesellschaft auf die ,Dynamik' und die ,Risikobereitschaft' ganz zu schweigen. Betroffen sind vor allem auch die Systeme der Sozialen Sicherung (Renten-, Kranken-, Pflege-, Arbeitslosen- und Unfallversicherung), wobei die Intensität der Auswirkung demographischer Verschiebungen nicht nur von der Funktion des jeweiligen Systems, sondern auch von seiner konkreten Ausgestaltung abhängt.

Die besondere Aufmerksamkeit, die bei der Diskussion der Konsequenzen des ,demographischen Wandels' für die Gesellschaft im Allgemeinen und die Systeme der Sozialen Sicherung im Besonderen (auch) in Deutschland der Gesetzlichen Rentenversicherung (GRV) gewidmet wird, hat sicher viele Gründe. Zu nennen wäre hierbei die Tatsache, dass die große Mehrheit der in Deutschland abhängig Beschäftigten in der GRV versichert ist. Ferner ist die Bedeutung dieser Mitgliedschaft sowohl in der Zeit der Erwerbstätigkeit als auch in der Rentenphase besonders ausgeprägt: Die Pflichtbeiträge (zumal bei kumulierter Betrachtung der sog. Arbeitnehmer- und Arbeitgeberanteile) stellen bedeutende - wenn nicht die bedeutendsten - Abzugsbeträge von der Bruttolohnsumme vieler Beschäftigter dar, so dass mögliche demographisch begründete Änderungen während der Aktivzeit finanziell besonders einschneidend sind. Noch viel größer ist die Bedeutung der GRV allerdings auf Seiten der Rentner, ist doch für einen Großteil dieser Gruppe die Rentenzahlung der bei weitem bedeutendsten Einkommensbestandteil.

Zusätzlich den gerade genannten Problembereichen, die aus den hohen Transfersummen der Rentenversicherung resultieren, sprechen aber auch systematische Gründe dafür, der GRV besondere Aufmerksamkeit bei der Betrachtung der ökonomischen Wirkungen einer, Alterung' der Gesellschaft zu widmen. So ist die GRV durch die Änderung der Altersstruktur der Bevölkerung auf zwei verschiedenen Seiten betroffen: Zum einen sinkt die Stärke der Erwerbsgeneration schon in recht naher Zukunft, was per se die Möglichkeit einer Verschlechterung der Bedingungen zur Aufbringung der Finanzmittel für die gestiegene Zahl von Rentenbeziehern an Bedeutung gewinnen lässt. Zum anderen verändert die kontinuierliche Zunahme der durchschnittlichen Rentenbezugsdauer das Zahlenverhältnis zwischen Mitgliedern der Aktivgeneration und Mitgliedern der Rentnergeneration zusätzlich zugunsten der Rentner.

Im Zentrum der vorliegenden Arbeit steht aber nicht die Frage, ob und wie die Rentenversicherung die absehbaren demographischen Belastungen ,überstehen' kann. Hierzu gibt es viele Prognosen, insbesondere über die Entwicklung der Beitragssätze bei unveränderter Ausgestaltung der Gesetzlichen Rentenversicherung. Die Ergebnisse dieser Untersuchungen - extrem verkürzt dargestellt - zei- 
gen, dass der Beitragssatz zur GRV in den nächsten Jahr(zehnt)en deutlich steigen wird. Das exakte Ausmaß der Steigerung lässt sich nur schwer prognostizieren dafür werden sowohl das Beitragsaufkommen der GRV als auch die ,Rentenverpflichtungen' von zu vielen Einflussfaktoren bestimmt, insbesondere von der Erwerbstätigenquote (resp. dem Anteil der Pflichtversicherten an allen Mitgliedern der Aktivgeneration), dem durchschnittlichen rentenversicherungspflichtigen Einkommen der Aktiven, der Arbeitslosenquote, der durchschnittlichen Rentenbezugsdauer etc. ${ }^{3}$ Ein entscheidender Faktor ist hingegen für den prognostizierten Zeitraum ausgesprochen genau abzuschätzen: die Entwicklung des Umfangs der Rentner- und der Aktivgeneration (bei gegebener ,Altersabgrenzung' dieser Gruppen). Die wesentlichen demographischen Entscheidungen, die diese Entwicklung in den nächsten Jahrzehnten prägen, sind gefallen, die meisten der späteren Beitragszahler sowie (fast) ${ }^{4}$ alle Rentner für den Prognosezeitraum sind bereits geboren. Eine Unbestimmtheit kann sich allerdings aus Wanderungsbewegungen ergeben. Ansonsten stimmen die Prognosen über die Stärke der jeweiligen Generationen qualitativ überein: Die Gesellschaft ,altert', das zahlenmäßige Verhältnis zwischen Mitgliedern der Aktivgeneration und der Rentnergeneration verschiebt sich eindeutig zugunsten der letzteren.

Nimmt die Zahl der (potenziellen) Beitragszahler zur GRV so drastisch, wie zu erwarten steht, ab, so bedeutet dies (c.p.) eine Erosion der Einkommenssumme, aus der - u.a. - die Rentenzahlungen bestritten werden müssen. Dies wiederum hat zur Folge, dass entweder die Höhe einer durchschnittlichen Rente (im Vergleich zum durchschnittlichen Bruttoeinkommen der Aktiven) abgesenkt, oder aber die durchschnittliche Beitragszahlung gesteigert werden muss. ${ }^{5}$ Die gerade geschilderte Problematik wird allerdings durch Produktivitätssteigerungen der Beschäftigten, also durch eine Steigerung des (realen) Pro-Kopf-Einkommens, so-

3 Zur Relativierung der Bedeutung verschiedener Einflussfaktoren für die prognostizierten Änderungen des Beitragssatzes zur Rentenversicherung vgl. SINN und THUM (1999). Sie zeigen für verschiedene Prognosen zukünftiger Beitragssätze zur in Deutschland bestehenden Gesetzlichen Rentenversicherung auf, dass der größte Teil der Prognoseunterschiede durch Differenzen in den erwarteten Belastungen der Aktiven durch Beitragsverpflichtungen zur Gesetzlichen Kranken- und Pflegeversicherung sowie Arbeitslosenversicherung erklärt werden kann, während „Variationen in den Detailannahmen zur Entwicklung der Bevölkerung, der Erwerbsquote, der Stillen Reserve und der Frühverrentung .. generell keine besonders starken Hebel [sind], um die Prognosen des Beitragssatzes zu beeinflussen“ (ebd., S. 14).

4 Die Einschränkung gilt wegen möglicher ,Frührentner' sowie dem Bezug von Witwen- und Waisen- sowie Erziehungsrenten; bei Betrachtung des kumulierten Rentenvolumens sind diese Posten aber zu vernachlässigende Größen.

5 Möglich ist (natürlich) auch eine Kombination der beiden geschilderten Ansätze. 
wie durch eine Ausweitung des Anteils der Rentenversicherungspflichtigen an den Mitgliedern der Aktivgeneration abgemildert.

Die Auswirkungen einer Verringerung der durchschnittlichen Kinderzahl pro Mitglied der Aktivgeneration mögen per se noch keinen Grund für eine besondere Berücksichtigung des Kinderhabens im Rahmen der Rentenversicherung liefern. Immerhin kann die Generation in toto dann in ihrer Altersphase entsprechend an der (u.U.) vergleichsweise geringeren Lohneinkommenssumme und einem entsprechend geringeren Beitragsaufkommen zur GRV beteiligt werden, also die finanziellen Konsequenzen der Entscheidung zugunsten vergleichsweise geringerer Ausgaben für die Erziehung einer nachfolgenden Generation dann im Alter realisieren. Diese Argumentation zugunsten einer ,Nicht-Beachtung' generativer Entscheidungen ist aber nicht mehr tragfähig, wenn es Unterschiede zwischen den Investitionen der einzelnen Mitglieder der Aktivgeneration in das Humankapital der nachfolgenden Generation gibt. Ohne eine Berücksichtigung dieser unterschiedlichen Verhaltensweisen bestehen erhebliche intragenerationale Umverteilungswirkungen infolge (sich intragenerational nicht ausgleichender), externer Effekte' des Kinderhabens: Während die Kosten des Kinderhabens primär von den Eltern getragen werden, profitieren auch z.B. Leute ohne Kinder im Alter von den Zahlungen der Kinder anderer Leute.

Die allokative Bedeutung des gerade Gesagten wäre gering, gäbe es nicht Reaktionen von Menschen auf (ökonomische) Umgebungsbedingungen. Von solchen ist jedoch auszugehen: Ökonomen sehen in ihrer Weltsicht in Menschen (auch) Humankapital, und somit werden von ihnen Zeugung, ,Aufzucht' und Erziehung bzw. Ausbildung von Kindern grundsätzlich (auch) als investive Akte modelliert. Diese Sichtweise widerspricht zwar traditionellen moralischen Positionen, die das Kinderhaben und die Kindererziehung als eigenwertig - und nicht ökonomischer Betrachtung zugänglich - ansehen. ${ }^{6}$ Doch die schon charakterisierte Entwicklung der Geburtenzahlen ist ein deutlicher Hinweis darauf, dass die idealistisch-moralische Sichtweise des Kinderhabens die Realität nicht erklärt (während auf ökonomischen Anreizkonstellationen basierende Ansätze eher zu einer Erklärung in der Lage sind). Die moralische Sichtweise mag für Viele weiterhin ein Ideal sein charakteristisches Merkmal von Idealen ist jedoch, dass die Realität ihnen nicht entspricht.

Vor diesem Hintergrund sollen in der vorliegenden Abhandlung einmal die Konsequenzen einer radikal-ökonomischen Sichtweise des Menschen als Humankapital mit Blick auf ein ,soziales', also durch weitgehende individuelle Zwangsmit-

6 Konrad Adenauer wird das Diktum zugeschrieben, Kinder hätten die Leute immer. 
gliedschaft gekennzeichnetes und im Umlageverfahren (UV) organisiertes Alterssicherungssystem verdeutlicht werden. Schwerpunkt hierbei ist die Frage, wie ein Rentenversicherungssystem in einer solchen Perspektive konsequenterweise zu entfalten wäre. Es besteht nicht unbedingt die Absicht zur Entwicklung einer politischen Empfehlung zur Umgestaltung des in Deutschland bestehenden Rentenversicherungssystems, auch wenn an mehreren Stellen konkrete praktische Implementierungsfragen in die Erörterung einbezogen werden. Auf der Basis der vorliegenden Arbeit (allein) politische Gestaltungsempfehlungen auszusprechen wäre voreilig, da eine Reihe von Fragen in ihr nicht thematisiert werden. So wird in ihr nicht diskutiert, inwieweit eine konsequente Umsetzung der - in einem engen Sinne - ökonomischen Sichtweise möglicherweise zu einer Verdrängung auf intrinsischer Motivation basierender Handlungen führt. ${ }^{7}$ Gerade beim Thema ,Familie' - und wegen einer hohen Bedeutung von Emotionen für den familiären $\mathrm{Zu}$ sammenhalt - kann eine besondere Bedeutung der Crowding-out-Problematik nicht ausgeschlossen werden. Allerdings ist ebenfalls zu beachten, dass die sich bei einer konsequenten ökonomischen Entfaltung der Humankapitalperspektive für die Einzelnen ergebenden Be- und Entlastungen dem recht nahe kommen, was sich unter weitgehend staatsfreien (,natürlichen') Bedingungen in der Menschheitsgeschichte für Kinder, Eltern und Großeltern an intergenerativen Belastungswirkungen ergeben hat. Vor diesem Hintergrund sollten die in dieser Arbeit entwickelten - durch eine Humankapitalperspektive nahegelegten - Reformüberlegungen nicht als bloße Gedankenspielerei abgetan werden.

Der Verweis auf eine familiäre Altersabsicherung verdeutlicht, dass zu Zahlungen in die GRV fähige und gewillte Kinder notwendige Voraussetzung für die Sicherung der Rente sind. Beeinflussen die individuellen Erziehungsleistungen der Eltern die Fähigkeit der Rentenversicherung zur Zahlung von Renten, so legt es die ökonomische Sichtweise nahe, solche Sachverhalte (auch) im Rahmen der Rentenversicherung zu berücksichtigen, wenn es deutliche Unterschiede in der Kinderzahl und dem Einkommenserzielungspotenzial der Kinder gibt. Außerdem hat sich die Ursache unterschiedlicher individueller Investitionen in das Humankapital der nachfolgenden Generation verändert: Konnte Kinderlosigkeit früher in vielen Fällen auf biologische Ursachen einerseits und ,Schicksalsschläge' andererseits zurückgeführt werden, war also nur in vergleichsweise geringem Umfang Folge einer , bewussten' Entscheidung gegen Kinder, so kann dies heute sicher so nicht mehr gesagt werden: Es ist vielmehr davon auszugehen, dass eine geringe individuelle Kinderzahl häufig nicht den Wünschen der (potenziellen) Eltern widerspricht, sondern vielmehr als explizit oder implizit akzeptierte Folge einer individuell bevorzugten Lebensgestaltung, in der Kindern eine vergleichsweise geringe Bedeutung zukommt, anzusehen ist. Ist Kinderlosigkeit aber in wesentlichen

7 Vgl. FREY (1997) und FREY et al. (1996). 
Teilen weniger ,Schicksal' als ,bewusste Entscheidung', so mag die Bedeutung einer Berücksichtigung des Kinderhabens bei der Festlegung der Rentenhöhe zunehmen, liegt doch bei individueller Kinderarmut zunehmend kein versicherbares Risiko mehr vor, sondern das gewünschte Ergebnis einer Wahlentscheidung (Endogenität der niedrigen Nettoreproduktionsrate). ${ }^{8}$

Aus der hier vorgestellten Perspektive ergibt sich die grundlegende Struktur der vorliegenden Arbeit: In Kapitel 2 werden Gründe für einen ,Kinderleistungsausgleich' insbesondere auch im Rahmen der Gesetzlichen Rentenversicherung vorgestellt. Dies erfordert zuerst eine kurze Beschäftigung mit den Aufgaben der GRV. Auch wenn im weiteren Verlauf der Abhandlung auf eine im Umlageverfahren organisierte Rentenversicherung (RV) Bezug genommen wird, ist gleichwohl doch auch die Frage zu behandeln, ob auch im Falle eines im Kapitaldekkungsverfahren (KDV) ausgestalteten Rentenversicherungssystems u.U. eine explizite Kinderberücksichtigung angemessen sein könnte. In Kapitel 3 werden unterschiedliche Möglichkeiten der Kinderberücksichtigung im Rahmen einer im Umlageverfahren ausgestalteten Rentenversicherung thematisiert. Kapitel 4 verdeutlicht die distributiven Implikationen einer stärkeren Internalisierung der externen Erträge erfolgreicher Kindererziehung im Rahmen einer umlagefinanzierten Rentenversicherung. In Kapitel 5 werden dann ein Ausgestaltungsvorschlag, der in den letzten Jahren gerade auf Seiten organisierter ,Elternvertreter' eine nicht unerhebliche Popularität gewonnen hat, nämlich der Ansatz von ERNSTJÜRGEN BORCHERT, sowie die Kinderberücksichtigung im gegenwärtigen deutschen Rentenrecht vorgestellt und analysiert. Am Ende steht eine Schlussbetrachtung, in der gefragt wird, ob von einer verstärkten Berücksichtigung von Kindern im Rahmen der Rentenversicherung wesentliche Auswirkungen auf das generative Verhalten ausgehen dürften, und wie die Chancen einer ,kinderfreundlichen' Rentenreform im politischen Prozess zu beurteilen sind (Kapitel 6).

8 Während im früheren Bundesgebiet 1955 12,4 v.H. der ehelich lebendgeborenen Kinder in der bestehenden Ehe die vierten, fünften etc. Kinder waren, beträgt der entsprechende Wert für 1996 5,0 v.H. (vgl. STAT. BUNDESAMT (1998), Fachserie 1, Reihe 1, S. 107, Tab. 9.9 - eigene Berechnungen). Ca. 20 v.H. der 1960 geborenen Frauen bleiben kinderlos (der Wert für den Geburtsjahrgang 1940 betrug 10 v.H.) (vgl. STAT. BUNDESAMT (1995), S. 120). 


\section{Normative und grundlegende institutionelle Ausgestal- tungselemente}

Im folgenden Abschnitt (2.1) wird zuerst auf Unterschiede zwischen einer freiwilligen und einer zwangsweisen Alterssicherung eingegangen. Anschließend (2.2) werden - in aller Kürze - Gründe für einen ,Kinderleistungsausgleich' erläutert, wobei dafür eine Abgrenzung dessen, was im Rahmen der vorliegenden Arbeit als ,Kinderleistungsausgleich' bezeichnet wird, erfolgt. Dann (2.3) wird thematisiert, warum eine solche Berücksichtigung elterlicher Erziehungsleistungen auch innerhalb des Systems der Gesetzlichen Rentenversicherung geboten ist, und nicht nur z.B. im Rahmen der Einkommensbesteuerung vorgenommen werden soll. Nachfolgend (2.4) werden mögliche Charakteristika und Aufgaben einer Rentenversicherung skizziert, die für die weitere Untersuchung Bedeutung haben. Im Anschluss daran werden mögliche Wirkungen einer Rentenversicherung auf die Geburtenhäufigkeit dargestellt (2.5). Das Kapitel wird abgeschlossen durch eine summarische Thematisierung der Bedeutung von Kindern im Rahmen einer im Kapitaldeckungsverfahren ausgestalteten Rentenversicherung (2.6).

\subsection{Zwangsweise vs. freiwillige Alterssicherung}

Losgelöst von der konkreten Ausgestaltung eines Rentenversicherungssystems ist zwischen freiwilliger und zwangsweiser Mitgliedschaft zu unterscheiden. Im Folgenden wird zuerst auf die (möglichen) distributiven Unterschiede der beiden gerade genannten Ausgestaltungsmöglichkeiten eingegangen. Anschließend wird ein häufig genannter Grund für eine pflichtweise Alterssicherung genauer betrachtet: eine hiermit erfolgende Verhinderung von ,Altersarmut' sich freiwillig nur unzureichend absichernder Individuen.

\subsection{1. (Mögliche) Unterschiede in den distributiven Wirkungen freiwilliger und zwangsweiser Alterssicherungssysteme}

Der unterschiedliche ,Zwangscharakter' einer Rentenversicherung ist vor allem bei der Betrachtung der distributiven Wirkungen der Ausgestaltung des Alterssicherungssystems bedeutsam. Bei freiwilliger Mitgliedschaft können bestehende distributive Wirkungen einer Versicherung, wie sie sich (u.a.) aus der konkreten Ausgestaltung der Berücksichtigung des ,Kinderhabens' im Rahmen der RV ergeben, vom Versicherten in seiner Entscheidung über einen ,Beitritt' berücksichtigt werden (was per se zwar nicht die Bereitstellung eines allokativ effizienten Angebots sicherstellt, bei jederzeit möglichem Marktzugang für neue Anbieter 
bzw. Angebote aber für einen Wettbewerb sorgen kann, der die Angebotspalette näher an das Ideal allokativer Effizienz führt). Gruppen, die sich als ,distributive Verlierer' der konkreten Ausgestaltung identifizieren lassen, haben bei Freiwilligkeit der Versicherung Anreiz zum Nicht-Beitritt in eine bestehende Versicherung (resp. zur Neugründung einer Versicherung). Bei Zwangsmitgliedschaft in einem in seinen wesentlichen Parametern gesetzlich vorgegebenen Alterssicherungssystem besteht eine solche Möglichkeit nicht. Ein voting with the feet ist dann nicht (bzw. nur unter Inkaufnahme sehr hoher Wanderungskosten) möglich.

$\mathrm{Da}$ in einen freiheitlichen Rahmen die Anwendung von Zwang gerechtfertigt werden muss, ${ }^{10}$ stellt sich die Frage nach möglichen Begründungsansätzen einer zwangsweisen Alterssicherung. Eine mögliche Rechtfertigung könnte sein, dass nur so für alle Interessierten ein akzeptabler Lebensstandard im Alter gesichert werden kann - insbesondere auch für Kinderlose. Eltern können sich innerhalb der Familie (teilweise) absichern - zumal bei justiziabler Pflicht zur Unterstützung bedürftiger Familienangehöriger. Eine entsprechende Möglichkeit haben Kinderlose nicht (oder nur in wesentlich eingeschränkterem Maße). Es ist jedoch zu beachten, dass aus der Abwesenheit eines Zwangssystems der Alterssicherung nicht folgt, dass nicht auch Kinderlose die Möglichkeit einer freiwilligen Absicherung für ihr Alter haben. Zwar können sie regelmäßig nicht damit rechnen, infolge von Mitgefühl (von Familienmitgliedern oder Fremden) im Alter versorgt zu werden, doch haben sie die Möglichkeit, während ihrer Aktivzeit ,Tauschmittel' für ihre Rentenphase zurücklegen, die sie dann im Alter gegen Güter des täglichen Bedarfs eintauschen. Insoweit reicht das Ziel, allen Interessierten die Möglichkeit einer Absicherung für das Alter zu geben, nicht zur Begründung eines Versicherungszwangs aus.

Dieser muss folglich mit spezifischen Nachteilen einer freiwilligen Alterssicherung begründet werden. Ein solcher ist eine eventuell unzureichende freiwillige Absicherung für das Alter (sei es in Form einer zu geringen Kinderzahl, sei es in Form einer zu niedrigen Kapitalakkumulation). Bei bestehender Sozialhilfe und bei in anderen Bereichen des gesellschaftlichen Lebens ausgeübtem Zwang (insbesondere bei zwangsweiser Eintreibung von Steuern) besteht dann die Gefahr einer Ausbeutung der Gesellschaft durch unterlassene Altersvorsorge: Rechnen die Individuen damit, dass die Gesellschaft Altersarmut auch dann nicht akzep-

9 Von den spezifischen Problemen des allokativen Versagens von Versicherungsmärkten infolge asymmetrischer Informationen und daraus resultierender adverser Selektion und moral hazard werde dabei abgesehen.

10 Allgemein zur Frage einer Rechtfertigung des mit sozialen Sicherungssystemen verbundenen Zwangs s.a. HACKMANN (1990). 
tiert, wenn sie in Kauf genommene Folge individueller Entscheidungen (über die Bildung von Human- und Sachkapital während der Aktivzeit) ist, so verringert dies die Hemmung Kinderloser, das Risiko einzugehen, infolge einer zu gering bemessenen Altersvorsorge von der Allgemeinheit, d.h. insbesondere von den dann Aktiven, die wiederum die Kinder anderer Mitglieder der Altengeneration sind, durch Sozialtransfers ausgehalten werden zu müssen. Für die Betroffenen existiert mithin im Effekt eine Form einer umlagefinanzierten Alterssicherung, nur gegenüber einer bewussten Ausgestaltung als Rentenversicherung mit Kinderberücksichtigung dadurch modifiziert, dass sich die Kinderlosen nicht an den Kosten der Heranziehung der nachfolgenden Generation (im Rahmen der Alterssicherung) beteiligen. ${ }^{11}$

Die vorangegangene Argumentation bezieht sich auf den Fall einer bewussten Unterabsicherung für das Alter. Aber auch Leute, die zwar Kinder haben, die aber zur Unterstützung ihrer Eltern in deren Altersphase nicht in der Lage sind (z.B. infolge geringen verfügbaren Einkommens, oder auch Tod der Kinder), oder aber deren Kapital infolge unglücklicher Umstände entwertet wird, mögen im Alter zu einer eigenständigen Sicherung eines minimalen Lebensstandards nicht in der Lage sein, sondern der Unterstützung durch die Gemeinschaft bedürfen. Insbesondere ist zu beachten, dass Kleinfamilien zur intrafamiliären Absicherung gegen erhebliche Einkommens- und Vermögensschwankungen, extreme Lang- bzw. Kurzlebigkeit etc. de facto nicht in der Lage sind. Berücksichtigt man dann auch noch die erheblichen Transaktionskosten eines einzelfall-basierten Absicherungssystems für bedürftige Alte, so mag dies zur Begründung einer institutionalisierten Alterssicherung dienen. ${ }^{12}$ Die vorgestellte Argumentation ist allerdings sicher nicht in der Lage, eine zwangsweise Absicherung für das Alter zu rechtfertigen, die über die Gewährleistung eines sozio-kulturellen Existenzminimums im Alter, jenseits dessen kein gemeinschaftlicher Unterstützungsbedarf mehr gesehen wird, hinausgeht. ${ }^{13}$

11 Zur Frage, inwieweit ein Verzicht auf Sozialhilfeanspruch bei gleichzeitiger Freistellung von der Sozialversicherungspflicht ein akzeptabler Weg sein könnte, vgl. HACKMANN (1990).

12 Entsprechendes gilt auch für den Fall, dass myopische Individuen die Bedeutung eines Einkommenstransfers in das Alter systematisch unterschätzen, um dann später zu ,Versorgungsfällen' zu werden. Die zwangsweise Absicherung hat für diese Individuen den Vorteil, sie zu einem vorausschauendem Verhalten zu bewegen. Voraussetzung für eine Vorteilhaftigkeit ist jedoch (zumindest), dass ,der Staat' die wahren Präferenzen der Versicherten besser kennt als diese selbst.

13 Außerdem kann argumentiert werden, dass ein wahrhaft liberales System auch die Entscheidung für Altersarmut toleriert, und nicht versucht, einen derartigen Lebensstil zu verhindern 
Abschließend sei auf einen weiteren Grund hingewiesen, der eine zwangsweise Altersabsicherung begründen mag: Es ist nicht sicher, dass eine private Rentenversicherung (Markt für Leibrenten) zustande kommt. Eine mögliche Ursache für ein ,Marktversagen' kann asymmetrische Information sein: ${ }^{14}$ Die Versicherungsnehmer sind im Durchschnitt besser als die potenziellen Versicherer über ihr jeweiliges Risiko informiert. $\mathrm{Zu}$ einem gegebenem Tarif werden also besonders schlechte Risiken (dies sind für einen Anbieter von Leibrenten Leute mit überdurchschnittlicher Rentenbezugsdauer, also - vereinfacht gesprochen - ,Langlebige') verstärkt Versicherungsschutz nachfragen. Dies führt dazu, dass Versicherungen eine vergleichsweise hohe Versicherungsprämie verlangen, so dass der Anteil der ,schlechten Risiken' an den Versicherten weiter zunimmt - es kommt zu adverser Selektion. ${ }^{15}$ Konsequenz eines solchen Prozesses kann es sein, dass es marktlich nicht möglich ist, ,guten Risiken' ein Versicherungsangebot zu unterbreiten, ohne Verluste in Kauf zu nehmen, da ein solches Angebot immer auch von schlechten Risiken genutzt würde, und somit dem Versicherer Verluste brächte - und dies, obwohl das Versicherungsangebot, würde es nur von den guten Risiken nachgefragt, für den Anbieter verlustfrei wäre. ${ }^{16}$ Somit kann möglicherweise nur ein Angebot unterbreitet werden, das fast ausschließlich von schlechten Risiken erstanden wird, da es eine vergleichsweise geringe Rentenhöhe für gegebene Beitragszahlungen vorsieht. Viele ,gute Risiken' entscheiden sich dann mithin für einen Verzicht auf Versicherungsschutz.

In einer solchen Situation ist es nicht auszuschließen, dass ein staatlicher Versicherungszwang sowohl den guten als auch den schlechten Risiken Vorteile bietet. Infolge des Versicherungszwangs besteht nicht mehr die Möglichkeit adverser Selektion. Die Versicherungspolice wird von guten und schlechten Risiken gleichermaßen gekauft; deren Anteile an den Versicherungsnehmern (resp. an der Versicherungssumme) unterscheiden sich nicht von ihren Anteilen an der Bevölkerung, die dem Versicherungszwang unterliegt. Einige ,gute Risiken' stellen sich dann möglicherweise nutzenmäßig besser, da sie eine Versicherungspolice zu günstigeren Konditionen als denen für ,schlechte Risiken' auf einem ,freien Markt' erwer-

(sei es durch Zwang zur Altersabsicherung in der Jugend, sei es durch zwangsweises Eintreiben von Mitteln zur Bekämpfung von Altersarmut bei anderen Mitgliedern der Gesellschaft).

14 Im Unterschied zu den vorherrschenden Auffassungen wird in HACKMANN (1990) jedoch bestritten, dass eine allokative Rechtfertigung auf diese Weise überzeugend gelingt.

15 Für das Folgende vgl. ROTHSCHILD und STIGLITZ (1976) sowie ECKSTEIN et al. (1985). Zur Bewertung vgl. auch HACKMANN (1990).

16 Formaler: Beschrieben ist die Nicht-Existenz eines trennenden Gleichgewichts auf dem Versicherungsmarkt für Leibrenten. 
ben; die ,schlechten Risiken' verbessern sich dadurch, dass die gemischte Police ihnen bessere Konditionen als der auf ,schlechte Risiken' abzielende marktliche Versicherungsvertrag bietet, da innerhalb der Versicherung eine Quersubventionierung schlechter durch gute Risiken erfolgt.

Bei unterschiedlicher Risikoaversion ansonsten innerhalb der jeweiligen Risikokategorie übereinstimmender Individuen bedeutet der Versicherungszwang z.B., dass die besonders risikoaversen guten Risiken sowie die schlechten Risiken tendenziell profitieren, während weniger risikoaverse gute Risiken zum Kauf eines Vertrages gezwungen werden, den sie freiwillig nicht erworben hätten - und damit die schlechten Risiken subventionieren. Die Rentenversicherung hat dann (u.a.) einer Ex-ante-Umverteilung zu Lasten der (wenig risikoaversen) guten Risiken zur Folge. Einige der bekanntesten Formen einer solchen Ex-ante-Umverteilung sind die Nicht-Berücksichtigung des Geschlechts und des Familienstandes, aber auch unterschiedlichen Gesundheitsbedingungen der Versicherten bei der Festlegung von Beitragshöhe und der Höhe der periodischen Rentenzahlung: Hier liegt eine Ex-ante-Umverteilung zugunsten, langlebiger' Versicherter vor (also z.B. zugunsten von weiblichen Versicherten), sowie zugunsten von Versicherten mit wirtschaftlich abhängigen Angehörigen, wenn diese nach dem Tod des Versicherten weiterhin eine Rente erhalten. ${ }^{17}$

\subsubsection{Rentenversicherung und ,Altersarmut'}

Eine schon implizit erwähnte Begründung einer Rentenversicherungspflicht ist das Argument einer Vermeidung von ,Altersarmut'. Änderungen in der Ausgestaltung der RV - wie eben eine zunehmende Berücksichtigung der Kindererziehung im Rahmen des Rentenversicherungssystems - haben (c.p.) regelmäßig eine Änderung der Rentenzahlungen oder aber Verschiebungen innerhalb der Gruppe der Rentenbezieher zur Folge: Was den einen (Kinderreichen) begünstigt, verringert die Rente eines anderen (Kinderarmen).

Für die vorliegende Arbeit wird davon ausgegangen, dass es nicht Ziel der GRV ist, Altersarmut von Versicherten (bzw. ihrer direkten Angehörigen) auch in Extremfällen zu vermeiden. Begründung für diese Annahme, die eine Senkung auch geringer Renten bei einer Umgestaltung des Systems (durch veränderte Berück-

$17 \mathrm{Zu}$ beachten ist, dass zumindest das Geschlecht des bzw. der Versicherten ohne jeglichen Kontrollaufwand festzustellen ist, so dass sich eine fehlende Differenzierung von Beitrag bzw. monatlicher Rente nicht mit der Vermeidung hoher Kontroll- und Verwaltungskosten resp. asymmetrischen Informationen begründen lässt. 
sichtigung von Kindern) zulässt, ist, dass weiterhin eine allgemeine - aus dem Steueraufkommen finanzierte - Sozialhilfe existiert, die einen minimalen Lebensstandard aller Mitglieder der Gesellschaft, also insbesondere auch der Rentner mit geringer Rente bei gleichzeitig geringem anderweitigen Einkommen und Vermögen, garantiert. Eine RV ist nicht per se in der Lage, auch im Falle sehr geringer Beitragszahlungen eine ,Mindestrente' zu zahlen. In der Ausgestaltung der GRV dominiert das Kausal- gegenüber dem Finalprinzip. Rentenzahlungen werden also - im wesentlichen - ohne Bedürftigkeitsprüfung vorgenommen. Für die Sozialhilfe ist hingegen eine Finalorientierung charakteristisch: Ziel ist die Sicherung eines minimalen Lebensstandards, unabhängig von den Gründen für das Nicht-Erreichen einer eigenständigen Sicherung dieses Lebensstandards. Vor der Hilfegewährung findet eine Überprüfung der Bedürftigkeit statt, die auch Nicht-Erwerbseinkommen und die Vermögensposition des Antragstellers (sowie enger Verwandter) einbezieht. Wäre die (generelle) Sicherung eines Sozialhilfeminimums Aufgabe der RV, so hätte dies entweder eine erhebliche Ausweitung der Kontrolle der Versicherten durch die Versicherung (Bedürftigkeitsprüfung, Verhinderung des Missbrauchs), oder einen drastischen Anstieg der Versicherungsansprüche mit Gefahr der Kollabierung des Systems (falls jeder, der einmal im Leben in die GRV eingezahlt hat, dafür im Alter Anspruch auf eine Rente mindestens in Höhe der Sozialhilfe hat) zur Folge. Ferner lässt sich argumentieren, dass nicht-ausreichendes Einkommen die Solidarität der Gesamtgesellschaft erfordert, und nicht nur die des Zwangskollektivs der Rentenversicherten. Letzteres wird umso bedeutender, je weiter die Bemessungsgrundlage der Rentenversicherungsabgabe von der (denen) des Steuersystems divergiert und je weniger sie durch staatliche Zuschüsse aus dem Steueraufkommen finanziert wird. Ist die Bemessungsgrundlage der GRV geringer als die des Einkommensteuersystems, so führt eine Übertragung allgemeiner Aufgaben (wie eben der Vermeidung von Altersarmut auch in besonderen Fällen) auf die GRV zu Belastungswirkungen, die nicht der Leistungsfähigkeit der jeweils Belasteten entsprechen. ${ }^{18}$

Als weiterer Grund für die Finanzierung von (faktischen) Sozialhilfeleistungen (auch) für Mitglieder der Rentenversicherung über Steuermittel und nicht über die Rentenversicherungsabgabe kann auf die Unterschiede in den Belastungskonzeptionen der beiden Systeme verwiesen werden: Bei Sozialabgaben erfolgt regelmä-

18 Eine geringere Bemessungsgrundlage der GRV reicht bei strikter Betrachtung nicht aus, Belastungsunterschiede in Abhängigkeit von der ,Zuordnung' der zur Leistung der ,sozialen Ausgaben' verpflichteten Stelle festzumachen: Bei interindividuell übereinstimmendem Verhältnis der beiden (potenziellen) Bemessungsgrundlagen hat die Wahl der Bemessungsgrundlage keinen Einfluss auf die ,Lastverteilung'. Aber gerade dieses ubereinstimmende Verhältnis ist bei Existenz von Beitragsbemessungsgrenzen, Existenzminima, Pauschalbeiträgen, einer Beziehung der Sozialversicherungsbeiträge (fast) nur auf Erwerbseinkommen etc. in Verbindung mit einer begrenzten Sozialversicherungspflicht nicht gegeben. 
Big eine proportionale Belastung des der Abgabe zu unterwerfenden Einkommens, das wiederum im Normalfall nur einen Teil der Gesamtheit der Einkommen einer Person ausmacht. Die Aufbringung von Steuern soll hingegen die (steuerliche) Leistungsfähigkeit der Individuen berücksichtigen: Die Abgrenzung des (einkommen)steuerpflichtigen Einkommens ist (normalerweise) weiter als die des rentenversicherungspflichtigen Einkommens, und die Belastung des steuerpflichtigen Einkommens erfolgt für die Einkommensteuer regelmäßig über einen progressiven Tarif. ${ }^{19}$ Dies hat zur Folge, dass bei einer Tragung der Aufwendungen zur Vermeidung von Altersarmut ausschließlich durch die Beitragszahler der Rentenversicherung eine weit höhere Belastung der Bezieher kleiner und mittlerer Einkommen erfolgt als bei einer Aufbringung über die Einkommensteuer, und zwar aus zwei Gründen: Zum einen ist gerade bei Beziehern kleiner und mittlerer Einkommen mit einem hohen Anteil von rentenversicherungspflichtigem Einkommen am Gesamteinkommen auszugehen, zum anderen ist die durchschnittliche marginale Belastung infolge der (arbeits-)einkommensproportionalen Beitragsbemessung höher als bei einer Aufbringung über die höhere Einkommen (marginal) stärker belastende Einkommensteuer.

Ein letzter Grund für eine Finanzierung allgemeiner sozialer Sicherungsausgaben aus dem Steueraufkommen liegt in der formalen Unterscheidung zwischen Steuern und Abgaben i.e.S.: Während Steuern zu entrichten sind, ohne dass dafür ein Anspruch auf eine unmittelbare Gegenleistung besteht, haben Abgaben (i.e.S.) eine Nähe zu dem Erwerb einer unmittelbaren Gegenleistung. Ein Problem bei dieser Trennung ist es allerdings, dass bei genauerer Überprüfung in der Praxis der heutigen deutschen Politik in vielen Fällen eine Vermischung von Abgaben (i.e.S.) und Steuern vorliegt: Als exemplarisches Beispiel hierfür sei auf gesetzlichen Zwang zum Kauf einer Rentenversicherungspolice zu einem Einheitspreis durch gute und schlechte Risiken verwiesen. Hätten die guten Risiken diese Police wegen fehlender individueller Beitragsäquivalenz freiwillig nicht erworben, so hat die Police dann wohl (zumindest) partiellen Steuercharakter. Die Abgrenzung wird jedoch noch komplizierter, wenn aufgrund eines Marktversagens ein privates Angebot für eine beitragsäquivalente Versicherung der ,guten Risiken' nicht zustande käme. Dann fehlt nämlich der Referenzpunkt für eine marktliche Bewertung des ,Abgabenteils' der Zwangsversicherung. Ferner stellt sich die Frage, ob nicht zumindest für risikoaverse , gute Risiken' auch ein ,Zuschlag' dafür berechnet werden darf, dass erst durch ein staatliches Eingreifen in Form einer Zwangsversicherung eine - für die Risikoaversen preiswerte - Versicherungsmöglichkeit geschaffen wird (vgl. 2.1.1).

19 Allerdings werden bei der Bemessung der Einkommensteuerschuld die Kosten der Einkommenserzielung steuermindernd berücksichtigt. 
Trotz der gerade genannten Abgrenzungsprobleme ist nicht zu erkennen, warum die Vermeidung von Altersarmut Nicht-Rentenversicherter von der Gesamtheit der Steuerzahler finanziert werden soll, die Vermeidung von Altersarmut Rentenversicherter aber ausschließlich vom (Zwangs-)Kollektiv der Rentenversicherten.

\subsection{Allgemeine Gründe für einen Kinderleistungsausgleich}

Nachfolgend werden zuerst zwei aus unterschiedlichen Perspektiven resultierende Ansätze für die Begründung einer Kinderberücksichtigung vorgestellt. $\mathrm{Da}$ beide Ansätze eine ökonomische Prägung haben, wird am Anschluss an ihre Präsentation auf die Frage eingegangen, inwieweit eine primär ökonomisch orientierte Betrachtung der Wirkungen des, Kinderhabens' überhaupt (moralisch) angemessen ist.

\subsubsection{Input- und outputorientierte Begründungsansätze}

Die Berücksichtigung von Kindern seitens der staatlichen Politik kann verschiedenen Zielen dienen, und hat dementsprechend ihren Niederschlag in unterschiedlichen Systemen resp. Ausgestaltungsformen zu finden: Zuerst zu nennen ist die Berücksichtigung der Tatsache, dass Kinder Kosten verursachen. Bei gegebenem Bruttoeinkommen sinkt die wirtschaftliche Leistungsfähigkeit von Steuerpflichtigen (u.a.) mit der Zahl der mit diesem Einkommen zu versorgenden wirtschaftlichen Abhängigen - also insbesondere auch (und gerade) mit der Zahl der Kinder. Aufgabe der (einkommen-)steuerlichen Kinderberücksichtigung ist die angemessene Berücksichtigung dieser unterschiedlichen wirtschaftlicher Leistungsfähigkeit in Form einer Anpassung der steuerlichen Belastung. Wer von seinem Einkommen Kinder zu unterhalten hat, der soll bei gegebenem zu versteuernden Einkommen (c.p.) weniger Einkommensteuer abführen müssen als jemand ohne solche Unterhaltsverpflichtung. Insbesondere ist Einkommen, das zur Sicherung des Existenzminimums wirtschaftlich Abhängiger benötigt wird, (einkommen-)steuerfrei zu stellen. ${ }^{20}$

Die einkommensteuerliche Freistellung des (kindlichen) Existenzminimums ist aber nicht ausreichend, entlastet sie doch nur Eltern(teile), die bei entsprechender Verringerung der steuerlichen Belastung zu einer Tragung (zumindest) des kind-

20 Das Gesagte gilt bei umfassendem Einkommensverständnis (in Anlehnung an des Reinvermögenszugangskonzept). Für eine Argumentation, dass eine einkommensteuerliche Freistellung des Existenzminimums für den Fall eines nicht-umfassenden Einkommensbegriffs möglicherweise nur eingeschränkt geboten ist, vgl. HACKMANN (1994). 
lichen Existenzminimums in der Lage sind. Ist letzteres nicht der Fall, so bedarf es weiterer staatlicher Mittel zur Sicherung der menschenwürdigen Existenz der Familienmitglieder - insbesondere in Form von ungebundenen oder gebundenen Transfers sowie ,Naturalleistungen'.

Die gerade vorgestellten Anforderungen an eine staatliche Kinderberücksichtigung sind ,inputorientiert' und beziehen sich somit auf die (minimalen resp. von der Gesellschaft als unabdingbar erachteten) Kosten der Kindererziehung. Sosehr die Gemeinschaft aus moralischen Gründen Interesse an der ,Inputseite' der Kindererziehung haben muss, sowenig kann sie ausschließlich diese betrachten. Immerhin ,investiert' sie selber in die Kinder (so z.B. durch Ausgaben, die (auch) den Kindern zugute kommen (Schul- und Ausbildungssystem, Bau/ Unterhalt von Spielplätzen etc.)), begibt sich folglich anderer Ausgabemöglichkeiten staatlicher Mittel (oder einer Senkung der Steuersätze). Außerdem können viele staatliche Ausgaben (u.a.) Folge nicht ausreichenden elterlichen Erfolgs bei der Kindererziehung sein (man denke nur an (Jugend-)Strafanstalten, dauerhafte Abhängigkeit von staatlichen Unterstützungsleistungen etc.). Das Steuer- und Abgabensystem selber knüpft regelmäßig am wirtschaftlichen Erfolg der Betroffenen an. Soweit der Erfolg elterlicher Erziehung also zu erhöhten zu versteuernden bzw. sozialversicherungspflichtigen Einkünften führt, partizipiert die Gesellschaft an den Ergebnissen (auch) elterlicher Handlungen. Konsequenz hiervon ist - in Abhängigkeit von der konkreten Ausgestaltung der jeweiligen Systeme - eine teilweise gesellschaftliche Aneignung der pekuniären Erträge individueller (elterlicher Vor-)Leistungen. ${ }^{21}$ Kommt es aber zu einer (nennenswerten) Sozialisierung der Erträge bei weithin bestehender individueller Tragung der Kosten (insbesondere auch der Opportunitätskosten in Form z.B. aufgrund von Kindererziehung entgangener Arbeitseinkommen), so mindert dies aus individueller (elterlicher) Sicht den Anreiz zu einer aus gesellschaftlicher Sicht effizienten Intensität der Kindererziehung $-^{22}$ es unterbleiben gesellschaftlich wünschenswerte (,rentable') Investitionen in Kinder, da sie sich individuell nicht ,rechnen' ${ }^{23,24}$

21 Die literarischen Hinweise hierzu sind Legion. Beispielhaft seien an dieser Stelle BALL (1984), BECKER und BARRO (1988), BECKER und MURPHY (1988), BERTHOLD (1992), BORCHERT (1993), BORCHERT (1994), HÖHN (1986), LAPAN und ENDERS (1990), LÜDEKE (1995), SCHMIDT et al. (1985), SCHMIDT-KALER (1978), SUHR (1990) und WEBER (1987) benannt.

22 Zur Wirkung der Einführung einer umlagefinanzierten Rentenversicherung, wenn zuvor die Alterssicherung nur durch ,Unterstützungsleistungen' der Kinder an ihre alten Eltern gewährleistet wurde, und ferner Kinder von den Eltern nicht nur als ,Investitionsgut' (investment good), sondern auch als ,Konsumgut' (,consumtion good' / Nutzenstifter) angesehen werden, vgl. HACKMANN (1990, S. 130ff.) und FELDERER (1992, insbes. S. $317 \mathrm{ff}$.). Der Eltern durch das Kinderhaben entstehende Nutzen verhindert hierbei einen vollständigen Verzicht auf Kinder. Durch die Einfuhrung des Umlageverfahrens kann die individuell ,optimale' Kinderzahl sin- 
Außerdem ist zu beachten, dass das Steuersystem nur an den ,Ist'-Größen anknüpft - nicht-monetäre Leistungen der Eltern zugunsten ihrer Kinder werden folglich steuerlich nicht (bzw. nur indirekt in Form einer unterlassenen Besteuerung ,entgangener Einkünfte') berücksichtigt. Wenn aber zwei verschiedene Arten der Kindererziehung, von denen die eine auf einem höheren Einsatz finanzieller Mittel beruht, die andere aber mehr auf dem Einsatz von Opportunitätskosten (z.B. eben durch Betreuung des Kindes durch die Mutter mit damit einhergehendem Verdienstausfall sowie ,Freizeitverzicht'), einen gleich hohen (gesellschaftlichen) Ertrag erbringen, so ist aus outputorientierter Sicht grundsätzlich eine unterschiedliche Förderung dieser beiden Methoden nicht zu rechtfertigen - sie verletzt das Ziel einer ,Neutralität'. ${ }^{25}$

Für die gerade angesprochenen Probleme kann ein Kinderleistungsausgleich ein Lösungsansatz sein: eine Beteiligung der Eltern an den gesellschaftlichen Erträgen der privat erbrachten Erziehungsleistungen. Ein solcher Kinderleistungsausgleich unterscheidet sich von den anderen Kinderberücksichtigungsmaßnahmen

ken. Ein theoretischer Nachweis, dass dies der Fall ist, ist ohne weiteres wohl nicht möglich; eine solche Beziehung, die sehr einleuchtend klingt, wird eher postuliert (vgl. z.B. HÖHN (1989), S. 217), oder aber unter restriktiven Annahmen über die Nutzenfunktion der Eltern, die Eigenschaften von Kindern etc. gewonnen - mit entsprechend geringer Verbindlichkeit der Aussage für eine Übertragung auf die ,reale Welt'. Und Verweise auf den in der Praxis zu beobachtenden Rückgang der Fertilität bei Ausbau des Rentenversicherungssystems lassen sich nur schwerlich als ,Nachweis' nutzen: Zum einen wäre erst einmal zu prüfen, ob die Verursachung nicht genau in die entgegengesetzte Richtung geht (mit sinkender durchschnittlicher Kinderzahl steigt die Bedeutung institutionalisierter Absicherung!), zum anderen gibt es eine Vielzahl weiterer Faktoren, die ebenfalls auf die durchschnittliche Kinderzahl Einfluss genommen haben dürften (Verstädterung, Verbesserung der Bildung (insbesondere der Frauen), verbesserte Möglichkeiten zur Beschränkung der Kinderzahl, verringerter Druck der Umwelt hin zu (mehr) Kindern, Rückgang religiöser Bindungen, Zunahme der Erwerbstätigkeit auf Seiten der Frauen etc.) und die ihrerseits zum Teil wiederum vom veränderten generativen Verhalten beeinflusst sein dürften. Vgl. hierzu auch die Diskussion in Abschnitt 2.5.

Vgl. BALL (1984).

24 Für eine Untersuchung des, Wertes' von Kindern in einem Land mit einem nur gering ausgeprägten sozialen Alterssicherungssystem sei auf die (Süd-)Korea-Studie AHNs (1995) verwiesen. Auf Grund der besonderen kulturellen Gegebenheiten, aber auch wegen des fließenden Übergangs der Argumentation von pekuniären hin zu nicht-pekuniären Beweggründen für das Kinderhaben sind die dortigen Ergebnisse allerdings mit Vorsicht zu interpretieren. Zur Bedeutung von Kindern als Möglichkeit intertemporalen Einkommenstransfers vgl. auch APPELBAUM und KATZ (1991).

,Neutralität' in allen Entscheidungsbereichen kann allerdings nicht alleiniges Ziel staatlichen Handelns sein: Insbesondere ist hier an Verbote ,intolerabler' Erziehungsmethoden zu denken - auch wenn diese ,erfolgreich' sind. Aufgabe der vorliegenden Arbeit kann und soll nicht eine exakte Abgrenzung solcher ,Erziehungsmethoden' sein. 
dadurch, dass er erfolgs- bzw. outputorientiert - und nicht erfolgsunabhängig und inputorientiert - ist, und, trotz seines Namens, die Eltern - und nicht das Kind als Bezugsperson nimmt.

\subsubsection{Exkurs: Zur Ökonomisierung}

Spätestens bei der Thematisierung der ,Leistungen' von Eltern mag sich eine Unterscheidung des in der Arbeit verfolgten ,ökonomischen' Zugangs von einem ,gefühlsmäßigen' ergeben. Die Konzentration auf die ökonomischen Aspekte bedeutet nicht, dass Entscheidungen über Kinderzahl, Ausgaben pro Kind, Gesamtausgaben, aber auch staatliche Aktivitäten zugunsten von Kindern bzw. deren Eltern etc. ausschließlich ökonomischer Rationalität unterliegen. Der ökonomische Datenkranz bildet jedoch einen bedeutenden Einflussfaktor für alle diese Entscheidungen. Wenn also in der vorliegenden Arbeit von der (individuellen oder gesellschaftlichen) ,Rentabilität' des ,Kinderhabens' gesprochen wird, so besagt das nicht, dass die individuellen Entscheidungen der Eltern ausschließlich im Sinne einer strengen ,betriebswirtschaftlichen Gewinnmaximierung' fallen. Doch auch bei Berücksichtigung der nicht-materiellen Erträge des Kinderhabens auf Seiten der Eltern werden die Kosten des Kinderhabens Einfluss auf die Entscheidungen haben. Nur bei reiner Reduktion des Menschen auf Instinkte - also unter Leugnung jeglichen Abwägens von Vor- und Nachteilen einer Handlung im Zuge eines Entscheidungsprozesses - blieben ökonomische Aspekte weitestgehend ohne Erklärungsgehalt für individuelle Entscheidungen (auch und gerade in Bezug auf das Kinderhaben). ${ }^{26}$ Wenn auch eine Erfassung aller externen Effekte des Kinderhabens und der konkreten Kindeserziehung nicht möglich ist, so wäre es doch die falsche Schlussfolgerung, auf eine Untersuchung der ökonomischen Konsequenzen zu verzichten. Dass es hierbei Raum für weitere (wissenschaftliche) Zugriffsmöglichkeiten auf ein so privates Thema wie die Familienplanung gibt, die abweichende Schwerpunkte setzen, spricht nicht gegen eine Thematisierung mit ökonomischem Schwerpunkt. Abschließend sei angemerkt, dass sich auch (und gerade) hinter Hinweisen, eine Ökonomisierung dieses Themas sei zu vermeiden, im Einzelfall eine Verfolgung ökonomischer Zielsetzungen verbergen mag. Eine Untersuchung der ökonomisch relevanten Zusammenhänge mag hierbei Hinweise darauf geben, wer von einer Tabuisierung ihrer Betrachtung möglicherweise profitiert. Ferner ist zwischen der Gewinnung von Kenntnissen über grundlegende wirtschaftliche Zusammenhänge bezüglich des (materiellen) Wertes des Kinderhabens für eine Gesellschaft und den daraus zu ziehenden Schlussfolgerungen zu unterscheiden: Auch - und gerade - in Kenntnis der involvierten $\mathrm{Zu}$ sammenhänge lässt sich eine Politik betreiben, die nicht ausschließlich ökono-

26 Wobei auch dann ökonomische Faktoren in der Form von ,Restriktionen' Bedeutung hätten! 
mischen Optimalitätskalkülen Rechnung trägt - dann aber in (besserem) Wissen um die Kosten einer solchen Politik.

\subsection{Institutionelle Möglichkeiten einer Berücksichtigung von ,Kindererziehungsleistungen'}

Da alle Kinder - mehr oder minder intensiv, erfolgreich etc. - erzogen werden, und da sich wesentliche Teile des Sozialisationserfolges in ihrer späteren pekuniären Renumeration manifestieren, auf die wiederum grundsätzlich sowohl die Besteuerung als auch die Systeme der Sozialen Sicherung ,zugreifen', bleibt zu klären, inwieweit eine gesonderte Berücksichtigung von Kindererziehungsleistungen außerhalb des Steuersystems angebracht ist, also z.B. in den verschiedenen Sozialversicherungssystemen erfolgen soll. ${ }^{27}$

27 Ohne dies im weiteren Verlauf der Arbeit zu thematisieren, wird die Systematik einer Anerkennung von Erziehungsleistungen ausschließlich auf elterliche Leistungen bezogen. Aus der unmittelbaren Betrachtung ausgeschlossen werden folglich Erziehungsleistungen durch Institutionen bzw. die sie repräsentierenden Personen (Kindergärtner, Lehrer, Aufsichtspersonal in Strafanstalten etc.), aber insbesondere auch die durch die ,Umgebung' (Freunde, Bekannte, Nachbarn etc.) erfolgenden Prägungen (Für die Bedeutung von , homogenen Nachbarschaften' für die Bildung etc. vgl. KREMER (1997).). Die Begrundung für diesen Ausschluss ist eher formaler als grundsätzlicher Natur: Wer eine Zurechnung von Erziehungsleistungen will, der muss diejenigen kennen, die diese Leistungen erbringen (Identifikationskriterium). Bei informellen Beziehungen ist dies regelmäßig spăter nicht mehr der Fall, so dass eine nachträgliche gesellschaftiche Anerkennung nicht praktikabel ist. Ferner ist in homogenen Gruppen grundsätzlich von einer gegenseitigen Erziehung auszugehen, so dass - cum grano salis - ein Ausgleich der Leistungen unterstellt werden kann (was einen Verzicht auf eine Berücksichtigung distributiv rechtfertigt, allokativ aber - wegen der Bereitstellung eines ,öffentlichen Gutes' nicht zu einer effizienten Bereitstellung führt). Schlussendlich ist zu betonen, dass die Eltern einen weitgehenden Einfluss auf das ihr Kind prägende Milieu haben (von der Einflussnahme auf einzelne Freundschaften bis hin zur Wohnort- und Schulwahl mit einer damit einhergehenden Vorauswahl, aber auch durch die Beeinflussung der kindlichen Präferenzbildung), so dass eine (indirekte) Zuordnung der ,Sozialisationswirkungen' zu den Eltern von daher auch methodisch vertretbar erscheint (vgl. HAVEMAN und WOLFE (1995), S. 1832). Bei denjenigen hingegen, die sich beruflich um die Erziehung ,Fremder' kümmern (eben z.B. Kindergärtner, Lehrer etc.), erfolgt die gesellschaftliche Anerkennung ihrer Leistungen grundsätzlich in Form der unmittelbaren Bezahlung für die erbrachte Tătigkeit. Die Tatsache, dass das Entlohnungs- und Anreizsystem in den ,Sozialisationsberufen' nicht unbedingt so ausgestaltet ist, dass es zu einer Belohnung außergewöhnlich erfolgreicher Erziehungsleistungen der Beschăftigten kommt, ändert an dieser grundsătzlichen Feststellung nichts. 


\subsubsection{Kinderleistungsausgleich ausschließlich im Rahmen der Einkommen- steuer?}

Der wohl augenfälligste Grund für eine gesonderte Berücksichtigung des Kinderleistungsausgleichs innerhalb der verschiedenen Systeme der Sozialen Sicherung ist die Tatsache, dass die den verschiedenen Systemen unterworfenen Wirtschaftssubjekte nicht übereinstimmen. Die Zahl der (potenziell) Einkommensteuerpflichtigen übersteigt die der in den anderen Systemen vertretenen Bürger deutlich. Ferner wird die Teilnahme am Einkommensteuersystem allen Bürgern unvermeidlich aufgezwungen. Die Systeme der Sozialen Sicherung kennen hingegen - unbeschadet einer recht weitgehenden Pflichtmitgliedschaft - allesamt Möglichkeiten, sich legal der Versicherungspflicht zu entziehen, aber auch die Möglichkeit einer freiwilligen Mitgliedschaft. Ferner unterscheidet sich die individuelle Höhe des der Einkommensteuer zu unterwerfenden Einkommens regelmäßig (aber nicht interindividuell gleichmäßig) von der Höhe des sozialversicherungspflichtigen Einkommens, und außerdem gibt es Unterschiede in der Belastungskonzeption ((direkt) progressiv vs. proportional zur Bemessungsgrundlage etc.).

Für eine gesonderte Kinderberücksichtigung spricht auch, dass eine solche Organisation (möglicherweise) die Einsicht stärkt, welche Bedeutung das Kinderhaben im allgemeinen und der jeweilige Erfolg elterlicher Erziehungsleistungen im besonderen für die einzelnen Systeme haben. Hierbei mag es zwischen den einzelnen Systemen Unterschiede geben, die sich dann jeweils auch in unterschiedlicher ,Kinderberücksichtigung' manifestieren sollten.

Die gerade vorgestellte Argumentationsleitlinie zugunsten einer Unterteilung der Kinderberücksichtigung auf einzelne Träger wird in ihrer grundsätzlichen Struktur nicht von der Tatsache berührt, dass wohl beim größten Teil der Bevölkerung das der Einkommensteuer unterworfene mit dem sozialversicherungspflichtigen Einkommen recht eng (positiv) korreliert ist. $\mathrm{Da}$, wo verschiedene - unabhängige Ziele verfolgt werden, bedarf es zu ihrer Erreichung einer entsprechenden Zahl unabhängiger Instrumente. Und die Zielsetzung des Kinderleistungsausgleichs unterscheidet sich - wie schon dargestellt (vgl. 2.2.1) - fundamental von denen des Steuer- und des Sozialhilfesystems.

Ein weiterer Hinweis auf die Sinnhaftigkeit eines Kinderleistungsausgleichs in den Systemen der Sozialen Sicherung ist, dass es qualitative Unterschiede zwischen Steuer- und Beitragszahlungen gibt (wobei letzteres nur für den Fall gilt, dass die Beitragszahlungen nicht falsch tituliert sind). Während mit höheren Beitragszahlungen zur GRV auch ein Anspruch auf eine höhere monatliche Rente 
verbunden ist, gilt Vergleichbares bei der Einkommensteuerzahlung nicht. ${ }^{28}$ Wenn Kinder nun eine gesonderte Bedeutung für die Sicherungssysteme haben, wäre eine reine Berücksichtigung ihrer Bedeutung hierfür im Rahmen des (Einkommen-)Steuerrechts mithin systematisch falsch. ${ }^{29}$

\subsubsection{Beitragsäquivalenz als Maßstab}

Wie schon erwähnt, ist es nicht sinnvoll, die unterschiedlichen Systeme der Sozialen Sicherung ,pauschal' zu betrachten. Vielmehr ist eine Untersuchung der Sinnhaftigkeit, der ,richtigen' Ausgestaltung und der Praktikabilität eines Kinderleistungsausgleichs für jedes der Systeme einzeln vorzunehmen. Hierbei ergibt sich das grundsätzliche Problem, dass für jedes der Systeme eine Nulllinie der ,Kinderneutralität' gesucht werden muss, um Förderungen von Kindern (also Leistungen der jeweiligen Institution zugunsten von Kindern in deren Jugendphase, die dann u.U. mit eventuellen ,Kinderleistungszahlungen' an die Eltern in späterer Zeit verrechnet werden müssen) ausfindig machen zu können.

In den folgenden Ausführungen wird die ,Beitragsäquivalenz ${ }^{30}$ als Maßstab einer ,kinderneutralen' Ausgestaltung genommen, d.h. Abweichungen der Kinderberücksichtigung von dem sich unter Beitragsäquivalenz ergebenden Niveau werden als ,Kinderförderung' (bzw. ,fehlende Berücksichtigung rentenmäßiger externer Effekte des Kinderhabens') interpretiert. Zu den Problemen eines solchen Ansatzes vgl. Abschnitt 2.1 .

28 Auch bei der Gesetzlichen Krankenversicherung (GKV) sind mit höheren Beitragszahlungen (fast) keine höheren Leistungsansprüche verbunden. Allerdings erfolgt bei der GKV eine (weitgehend) beitragsfreie Mitversicherung der Kinder Versicherter, so dass hier in nicht unbedeutendem Maße eine Mittelverausgabung zugunsten von Kindern erfolgt.

Für die Forderung, den ,Familienlastenausgleich' derart auszubauen, dass sich eine gesonderte Berucksichtigung des Kinderhabens bei der Altersrente erübrigt, vgl. Gutachten des Wissenschaftlichen Beirats beim Bundesministerium der Finanzen vom 01. Juni 1971, wiedergegeben in: WISSENSCHAFTLICHER BEIRAT ... (1974).

Beitragsäquivalenz wird hier verstanden als Übereinstimmung der Versicherungsprämie mit dem Erwartungswert der Versicherungsleistungen. Aufgrund des Erkenntnisinteresses ist hierbei auf die einzelnen versicherten Individuen Bezug zu nehmen - eine ,Globaläquivalenz' für die Gesamtheit der Mitglieder einer Alterskohorte (oder gar eine rein technische Übereinstimmung des Barwerts der erwarteten Einnahmen und Ausgaben) ist zur Aufdeckung intragenerationaler Umverteilungsprozesse nicht tauglich. (Für eine Diskussion der ,Beitragsäquivalenz' vgl. WAGNER (1984), insbes. S. 55f., 62f. und 81ff.) 


\subsubsection{Ausgestaltung einer Kiŕderberücksichtigung bei Existenz mehrerer Alterssicherungssysteme}

Unabhängig vom schon thematisierten (vgl. 2.1) Zwangscharakter einer Rentenversicherung besteht die Möglichkeit einer parallelen Existenz mehrerer eigenständiger Alterssicherungssysteme. Der einzelne Versicherungspflichtige kann hierbei entweder ein unmittelbares Wahlrecht zwischen den einzelnen Versicherungen haben, oder aber - z.B. infolge seiner Berufswahl - zur Mitgliedschaft in einem dieser Alterssicherungssysteme gezwungen sein. Zur Konkretisierung sei für Deutschland an die Alterssicherung für Beamte erinnert, aber auch an die Möglichkeit z.B. von Ordensgemeinschaften, eine eigenständige Absicherung ihrer Mitglieder zu betreiben.

Die Frage, ob es nur eine generelle Pflichtmitgliedschaft in einem Alterssicherungssystem gibt, oder aber den Zwang zur Mitgliedschaft in einem konkreten System, ist von grundsätzlicher Bedeutung für die möglichen distributiven Wirkungen der Gesamtheit der Alterssicherungssysteme: Bei Versicherungszwang, aber Wahlfreiheit zwischen verschiedenen Versicherungsgesellschaften (die Freiheit in der vertraglichen Gestaltung wesentlicher Versicherungselemente haben) besteht für die Versicherungspflichtigen die Möglichkeit, für sie besonders unattraktive Angebote zu meiden, was die Fähigkeit der Versicherungen, erkennbare Umverteilungen zwischen einzelnen Versichertengruppen vorzunehmen, mindert. Für die weitere Argumentation bezüglich der Kinderberücksichtigung bei mehreren getrennten Alterssicherungssystemen ist die Unterscheidung zwischen der Pflichtmitgliedschaft in einem bestimmten System oder aber allgemeiner Versicherungspflicht aber nicht weiter erheblich: Hier sind die Kinder für das konkrete Alterssicherungssystem bedeutsam, dessen Mitglied sie - warum auch immer werden. Insoweit ist es angebracht, dass die Kinderberücksichtigung nicht von dem Rentenversicherungsträger geleistet wird, dessen Mitglied die Eltern sind, sondern von dem, dessen Mitglied das Kind in seiner Aktivphase wird.

Die daraus entstehenden verwaltungsmäßigen Komplikationen können eine Zusammenarbeit unterschiedlicher Rentenversicherungen bei der Auszahlung eventueller ,Renten für Kindererziehung' nahelegen, bei der zwar die Rente für jeden Versicherten nur von seinem jeweiligen Versicherer ausgezahlt wird, dieser aber die kinderbezogenen Rententeile von dem Versicherer, dessen Mitglied das jeweilige Kind ist, einzieht. Bei sehr großer Ähnlichkeit der Ausgestaltung der einzelnen Rentenversicherungen sowie der Struktur ihrer Versicherten kann es ferner aus praktischen Gründen naheliegen, je nach Ausgestaltung der Kinderberücksichtigung eine ,Eigenständigkeit' der Kinderberücksichtigung zu implementieren, bei der jede Versicherung für alle Kinder eines Versicherten, die Mitglieder einer der Rentenversicherungen des Verbundes werden, die Finanzierung der kin- 
derbezogenen Rente übernimmt: Der Grund hierfür liegt dann aber nicht in der systematischen Angemessenheit eines solchen Verfahrens, sondern in den günstigen Verwaltungskosten in Verbindung mit der geringen ,Ungenauigkeit' aufgrund der genannten Konstellationen.

In der bisherigen Argumentation wurde unterstellt, Kinder leisteten im Rahmen einer Beitragszahlung innerhalb eines Rentenversicherungssystems einen unmittelbaren Beitrag zur Sicherung der Rentenzahlung an die in dieser Versicherung abgesicherten Mitglieder der Altengeneration. Implizit wurde damit auf die Organisation des Systems im Rahmen einer umlagefinanzierten Ausgestaltung abgezielt: Die Beiträge werden sofort zur Zahlung von Renten herangezogen (zu den Kennzeichen eines Umlageverfahrens vgl. näherhin Kap. 3, insbes. 3.1.1). Zielt die grundsätzliche Ausgestaltung des Rentenversicherungssystems hingegen auf eine eigenständige Absicherung der Mitglieder jeder Generation für das Alter durch Aufbau eines Kapitalstocks, aus dessen Veräußerung die Versicherten ihren Alterskonsum bestreiten, ab, so ist eine derartige Argumentation nicht mehr statthaft: Nach dem eigenen Anspruch der Versicherung bedarf es zur Aufrechterhaltung des Versicherungsschutzes keiner Beiträge der nachfolgenden Generation (für eine Problematisierung dieses Ansatzes vgl. 2.6). Insoweit erscheint es für eine derartig ausgestaltete Rentenversicherung eher angemessen, auf Zahlung kinderbezogener Renten an die Eltern der Mitglieder zu verzichten.

Eine besondere Bedeutung erhält das gerade Dargestellte dadurch, dass in Deutschland nicht nur viele berufsständische Absicherungssysteme auf Basis des Kapitaldeckungsverfahrens existieren, sondern auch eine gesonderte Alterssicherung der Beamten. Diese basiert weder auf einem expliziten Umlageverfahren innerhalb der Gruppe der Beamten, noch wird ein Kapitalstock aufgebaut, aus dem die Pensionsansprüche pensionierter Beamter bestritten werden. Vielmehr erwirbt jeder Beamte im Rahmen seiner Tätigkeit einen Pensionsanspruch - der möglicherweise zu Lasten einer niedrigeren Besoldung in der Aktivzeit geht. Die Tatsache einer eigenständigen Absicherung jeder einzelnen Beamtengeneration entspricht eher einem Kapitaldeckungsverfahren, der fehlende Kapitalstock lässt eher auf ein Umlageverfahren schließen.

Das gerade Gesagte legt nahe, dass für Kinder, die Beamte werden, kein systematischer Grund besteht, ihren Eltern einen kinderbezogenen Rentenanspruch zu gewähren. Ob Fürsorgeerwägungen, oder auch die Vermeidung einer adversen Selektion (Attrahierung nicht am Wohlergehen der Eltern Interessierter) staatliche (Renten-)Zahlungen an die Eltern von Beamten nahelegen, ist hier nicht weiter zu diskutieren. 
Für die weiteren Untersuchungen wird aus Gründen der Übersichtlichkeit und analytischen Bequemlichkeit eine weitgehende Zwangsmitgliedschaft in einem einheitlichen Rentenversicherungssystem unterstellt. In ihrer Grundtendenz entspricht diese Annahme der in Deutschland - aber auch vielen anderen Industrieländern - bestehenden Ausgestaltung. ${ }^{31}$ Entsprechend werden Kinder, die nicht Mitglieder dieser einheitlichen Rentenversicherung werden, in der weiteren Analyse nicht weiter beachtet; insbesondere begründen sie für ihre Eltern keine kinderbezogenen Renten.

\subsection{Charakteristika einer Rentenversicherung}

Schwierigkeiten bei der Analyse der Bedeutung von Kindern für die GRV ergeben sich aus dem Zusammentreffen vieler verschiedener Umverteilungs- und ,Kinderberücksichtigungsmechanismen', die sowohl auf der Ebene der Eltern als auch ihrer Kinder innerhalb deren späteren Erwerbslebens (und Rentnerdaseins) anzutreffen sind, und die noch dazu durch die intergenerationale Interdependenz in ihrer Unübersichtlichkeit weiter verstärkt werden. ${ }^{32}$ Es ist zu betonen, dass gerade für die GRV bei allen potenziellen Umverteilungen besonders zwischen in-

31 Zwar ist es in Deutschland möglich, sich der zwangsweisen Alterssicherung durch ,Selbstständigkeit' zu entziehen, doch sind die damit verbundenen (nicht-pekuniären) Kosten häufig prohibitiv hoch.

32 Zur Verdeutlichung der Probleme werde hier kurz auf ein Zitat Paul DEMENYs (1986, S. 447) zurückgegriffen (Hervorhebungen im Original): „Präferenzen zu Gunsten von Kindern und zu Lasten von Steaks bedeuten an sich kein Bevölkerungsproblem. Ein Bevölkerungsproblem besteht, wenn meine Präferenz für Kinder Ihren Zugang zu Steaks mindert, oder um ein vielleicht passenderes Beispiel ... zu wählen, es besteht ein Bevölkerungsproblem, wenn Ihre Präferenzen für keine Kinder meinen Anspruch auf ein gesichertes Pensionsrecht gefährden. Mit anderen Worten, wir haben ein Bevölkerungsproblem, wenn mit demographischem Verhalten externe Effekte verbunden sind." Zumindest das erstgenannte Beispiel ist fragwlirdig: Es wird hier ein pekuniärer externer Effekt beschrieben - eine erhöhte Nachfrage nach Steaks erhöht (c.p.) deren Preis, erschwert also den Erwerb. In der Diktion DEMENYs wäre es wohl angemessen, eine Präferenzänderung hin von der ,Kindererziehung' hin zum ,Steakessen' mit einhergehender Verteuerung von Steaks ebenfalls als ,Bevölkerungsproblem' zu bezeichnen (Allgemeiner kann gesagt werden, dass jede Nachfrage von Menschen kommt, und die so induzierte Preiserhöhung gegenuber dem Fall ohne diese Nachfrage Ausdruck eines ,Bevölkerungsproblems' ist - allerdings sind dieselben Leute, die als Nachfrager so kritisch gesehen werden können, als Produzenten an der Minderung von Knappheit beteiligt.). Und auch das zweite Beispiel ist so nicht ganz unproblematisch: Wird z.B. das gegenwärtig in Deutschland bestehende Rentenversicherungssystem betrachtet, so , leidet' der Einzelne potenziell unter der Kinderlosigkeit Dritter - dies gilt aber auch fur Leute, die selber kinderlos sind, so dass ein $A n$ spruch darauf, dass andere Leute Kinder haben, die dann die Altersversorgung der Kinderlosen sichern, zumindest fragwürdig erscheint. 
tra- und intergenerationalen Be- bzw. Entlastungswirkungen einzelner Maßnahmen $\mathrm{zu}$ unterscheiden ist. ${ }^{33,34}$

Bevor auf die Einzelheiten der Kinderberücksichtigung eingegangen wird, werden verschiedene allgemeine Aspekte der Ausgestaltung eines Rentensystems kurz thematisiert. Diese vorangehenden Ausführungen sollen der Verdeutlichung der Zielrichtung der vorliegenden Arbeit dienen, und durch Benennung weiterer Aspekte, die in der Arbeit nicht näher behandelt werden, verbleibende ,Ausbaumöglichkeiten' andeuten.

\subsubsection{Die zwei Grundaufgaben einer Rentenversicherung}

Die bestehenden Rentenversicherungssysteme zeichnen sich durch zwei voneinander grundsätzlich - bei allen Problemen, die im Detail auftreten - zu trennende Teilbereiche aus: Einerseits dient die Rentenversicherung als Mechanismus des Kaufkrafttransfers von der Zeit der Erwerbstätigkeit in die Altersphase, andererseits der (finanziellen) Versorgung der Hinterbliebenen im Falle des Todes des Versicherten und der Absicherung im Falle einer Berufs- bzw. Erwerbsunfähig-

33 Vgl. BREYeR (1990), S. 129 sowie FEUChT (1995), S. 227.

34 Gerade dieser Aspekt wird von vielen Autoren vernachlässigt bzw. nicht einmal erwähnt. Typischer Ausdruck einer solchen Vereinfachung ist z.B. die Ablehnung einer Kinderberücksichtigung in Form einer mit der Zahl der Kinder (c.p.) steigenden Rentenhöhe mit dem Argument, dann müssten gerade die Kinder dafür, dass ihre Eltern Kinder hatten, (c.p.) (wegen der gestiegenen Rentenansprüche bei rentensteigender Kinderberücksichtigung) höhere Rentenbeiträge entrichten, was doch nicht richtig sei (Für eine solche Argumentation vgl. DINKEL (1981), FRITZSCHE (1986), GAILLINGER (1994) sowie PRINZ und KLANBERG (1985); als beispielhaftes Zitat: „Wenn die Bundesregierung .. ,anrechnet' ..., dann bedeutet das im Klartext am Beispiel des Babyjahres, daß den Kindern der bedachten Eltern diese Last noch zusätzlich auferlegt wird!" (BORCHERT (1993), S. 168). Im Rahmen dieser Argumentation wird eine Umverteilung der (kurzfristig bei konstantem Beitragssatz gegebenen) Rentensumme von Rentenbeziehern, die keine Kinder erzogen haben, hin zu denjenigen, die Kinder erzogen haben, ausgeschlossen. Bei einer derartigen Ausgestaltung wären jedoch die einzahlenden Kinder nicht geschädigt, sondern tendenziell bevorzugt: Das ihnen abgenommene Geld käme zu größeren Teilen ihren eigenen Eltern zugute. Inwieweit das Geld z.B. in Form höherer Erbschaften oder sonstiger familiärer Einkommens- bzw. Vermögensübertragungen auch den Kindern zufließt, hängt u.a. vom Ausmaß des innerfamiliären Altruismus, aber auch von der Bedeutung möglicher finanzieller Restriktionen (Fähigkeit zur Anpassung des Konsumplans an einen als ,ideal' empfundenen) während der Aktivzeit der Eltern ab (vgl. dazu BECKER (1974) mit dem ,rotten kid theorem'; ALTONJI et.al. (1997), BECKER und MURPHY (1988), BERNHEIM (1991), BERNHEIM und STARK (1988), BERNHEIM et al. (1985), BRUCE und WALDMAN (1990), COX und JAKUBSON (1995), HESS und ORPHANIDES (1996), LAITNER und JUSTER (1996), PEROZEK (1998), STRAWCZYNSKI (1994), WILHELM (1996).). 
keit. ${ }^{35}$ Der erstgenannte Teil kann - cum grano salis - als ,Sparfunktion' angesehen werden, obwohl sich auch hier infolge der unterschiedlichen Restlebensdauer der Versicherten nach Erreichen des normalen Renteneintrittsalters Unterschiede in der Höhe der kumulierten Rentenzahlungen ergeben. Da eine Zusicherung einer regelmäßigen Rentenzahlung für die Zeit nach Erreichen einer vorgegebenen $\mathrm{Al}$ tersgrenze gegeben wird, kann somit eine Versicherung für den Fall überdurchschnittlicher Langlebigkeit unterstellt werden.

Während für diesen ersten Teilbereich der Familienstand des Versicherten per se unbedeutend ist, ${ }^{36}$ kann dies für den zweiten Bereich nicht gesagt werden. Hier tritt der Versicherungscharakter deutlicher hervor, und der Wert der Versicherung ist (u.a.) abhängig vom Familienstand: Nur da, wo es vom Versicherten finanziell abhängige Personen gibt, bestehen über den Tod des Versicherten hinaus Zahlungsverpflichtungen der RV..$^{37,38}$

Der Schwerpunkt der folgenden Analysen liegt in der ,Sparfunktion' der Rentenversicherung. Als Grund hierfür sei auf die finanziell weit höhere Bedeutung dieses Teils verwiesen werden, die noch dazu tendenziell - infolge einer zunehmenden eigenständigen Sicherung der Hinterbliebenen in Form eigener Rentenansprüche oder Erwerbseinkommen - zunimmt.

\subsubsection{Kinderberücksichtigung in der Rentenversicherung - eine ,versicherungsfremde Leistung'?}

In den letzten Jahren sind in Zusammenhang mit der Gesetzlichen Rentenversicherung ,versicherungsfremde Leistungen' zunehmend in die Diskussion geraten. Häufig erfolgt bei der Abgrenzung dessen, was ,versicherungsfremde Leistungen'

${ }^{35}$ Eine exakte Trennung der beiden Teilbereiche ist realiter problematisch ist - so im Falle einer Erwerbsunfähigkeit kurz vor Erreichen des Rentenalters.

36 Wenn - wie üblich - die Höhe der monatlichen Rentenzahlung nicht vom Familienstand des Empfängers abhängt.

37 Auch hier tritt die ,Interdependenz' der beiden Teile der Rentenversicherung deutlich hervor: Die finanzielle Absicherung von Angehörigen eines verstorbenen Beziehers einer Regelaltersrente fällt in der hier vorgenommenen Unterteilung in den zweiten Teil - die Nähe zur Regelaltersrente ist aber unubersehbar.

38 Inwieweit bei der Absicherung des finanziellen Risikos von Berufs- resp. Erwerbsunfähigkeit der Familienstand bedeutsam ist hängt davon ab, ob er die Höhe der jeweiligen Zahlungen der Versicherung beeinflusst. 
darstellt, eine Orientierung an den im Sozialgesetzbuch (SGB) festgelegten Leistungen, für die die Rentenversicherung einen Bundeszuschuss erhält: So werden Aufwendungen nach dem Fremdrentenrecht eindeutig zu den versicherungsfremden Leistungen gerechnet (SGB VI $\S 291$ b), wie auch verschiedene, einigungsbedingte' Zahlungen (SGB VI § 291c).

Eine besondere Bedeutung erhalten eventuelle versicherungsfremde Leistungen im Rahmen der Rentenversicherung durch die weitgehende Pflichtmitgliedschaft, die eine Nicht-Mitgliedschaft in vielen Fällen mit prohibitiv hohen Vermeidungskosten belegt. Der Versicherungspflichtige hat mithin keine (nennenswerte) Möglichkeit, sich der Finanzierung, versicherungsfremder Leistungen' zu entziehen. Noch dazu erfolgt diese Finanzierung in einer Art und Weise, die zu einer vergleichsweise höheren Belastung niedriger Einkommen führt, als sie im Falle einer Aufbringung der Mittel über einer Einkommensteuer anfiele (vgl. 2.3).

Zur Abgrenzung dessen, was ,versicherungsfremde Leistungen' sind, wird ein Referenzmaßstab benötigt, der angibt, was die ,normalen Leistungen' der GRV darstellt. Eine mögliche Abschätzung der Summe versicherungsfremder Leistungen der Rentenversicherung ergibt sich durch die Addition der positiven Differenzen zwischen den Beitragszahlungen der Versicherten und dem Erwartungswert der Versicherungsleistungen an diesen Personenkreis. ${ }^{39}$ Hierbei erfolgt keine exakte Festlegung dessen, was ,versicherungsfremde Leistungen' darstellt, sondern nur eine Bewertung des ,Gesamtangebots' der Versicherung für den einzelnen Versicherten.

Die vorgestellte Leitlinie ist problembehaftet: Insbesondere ist auf den schon thematisierten (vgl. 2.1.1) Fall eines fehlenden marktlichen Referenzmaßstabs bei Marktversagen hinzuweisen, der eine Abschätzung der jeweiligen Höhe versicherungsfremder Leistungen wesentlich erschwert. ${ }^{40}$ Außerdem ist z.B. das deutsche Rentenversicherungssystem durch eine Vielzahl von Ex-ante-Umverteilungen zwischen verschiedenen Personengruppen gekennzeichnet (z.B. infolge der NichtBerücksichtigung von Informationen über das Geschlecht der Versicherten, ihren Beruf und ihren Gesundheitszustand), so dass Versicherte mit unterschiedlichem Erwartungswert der Rentenbezugszeit für einen übereinstimmenden monatlichen Rentenanspruch einen gleich hohen Beitrag zahlen müssen, obwohl sie unter-

39 Bei genauerer Betrachtung wären die ,normalen`Verwaltungskosten als ,nicht-versicherungsfremd' zu berlucksichtigen.

40 Ferner sind real zu beobachtende Versicherungsmärkte kaum durch ,vollständige Konkurrenz' gekennzeichnet, so dass durch ihre Beobachtung kein Aufschluss auf die exakte aktuarisch faire Versicherungsprämie gewonnen werden kann. 
schiedliche Erwartungswerte der (abdiskontierten) Summe der Rentenzahlungen haben. ${ }^{41}$ Mithin ist auch hier die Angabe eines ,Referenzmaßstabs', von dem Abweichungen als ,versicherungsfremde Leistungen' charakterisiert werden können, nur schwerlich möglich. ${ }^{42}$

Trotz der gerade genannten Probleme erscheint der Vergleich der Beiträge mit denjenigen, die die Versicherten auf einem Versicherungsmarkt für eine entsprechend ausgestaltete Versicherung zu zahlen hätte, als sinnvoller Maßstab zur Erkennung (des Wertes), versicherungsfremder Leistungen', ließe sich doch unter Konkurrenzbedingungen eine nennenswerte Quersubventionierung schlechter Risiken kaum durchhalten: Diejenigen, die zu den Netto-Zahlern der Umverteilungsmaßnahmen gehörten, könnten in eine Versicherung ohne solche Umverteilung wechseln, so dass - unter einfachen Bedingungen - eine Versicherung mit Ex-ante-Umverteilung nicht überlebensfähig wäre. ${ }^{43}$

Ein besonderes Problem der Heranziehung der Konditionen entsprechender privatwirtschaftlich angebotener Rentenversicherungen als Referenzmaßstab zur Feststellung, versicherungsfremder Leistungen' einer im Umlageverfahren organisierten Rentenversicherung ist das unterschiedliche Organisationsprinzip: Private Rentenversicherungen sind regelmäßig durch das Kapitaldeckungsverfahren gekennzeichnet, die in Deutschland existierende Gesetzliche Rentenversicherung jedoch durch ein Umlageverfahren. Wie noch zu thematisieren (vgl. 2.6), ist eine gesonderte Kinderberücksichtigung (im Altersrententeil) bei dem Ideal einer eigenständigen Absicherung aller Mitglieder einer Generation, wie es dem KDV zugrunde liegt, nicht geboten. Sie würde unter Konkurrenzbedingungen auch nicht angeboten, wäre sie doch nur für Versicherte mit Kindern (bzw. überdurchschnittlicher Kinderzahl) interessant, während sie von kinderlosen (und kinder-

41 Zur ,Umverteilung im Rahmen der Gesetzlichen Rentenversicherung' vgl. WAGNER (1984), S. 166 - 188. Dort auch insbesondere Hinweise zur Unterscheidung inter- und intragenerationaler Umverteilung.

42 Theoretisch ist es möglich, dass alle Versicherten einerseits Begünstigte und andererseits Finanzierer versicherungsfremder Leistungen im Rahmen der Rentenversicherung sind, und sich diese beiden Elemente in ihrer finanziellen Be- resp. Entlastung gerade ausgleichen. Im Rahmen der obigen Abgrenzung werden dann keine ,versicherungsfremden Leistungen' ausgewiesen.

43 Auf Basis dieser Angrenzung wäre eine sich nicht in unterschiedlicher Beitragshöhe manifestierende Hinterbliebenenabsicherung als versicherungsfremde Leistung anzusehen, ebenso verschiedene ,Sonderleistungen' der Rentenversicherung (Rente nach Mindesteinkommen, Anrechnungszeiten etc.). Für eine entsprechende Einschätzung vgl. BERTHOLD und SCHMID (1997); vgl. auch WERDING (1998), S. 205ff. 
armen) Versicherten gemieden würde. Unter analytisch bequemen Bedingungen schlössen sich Versicherte mit gleicher Kinderzahl etc. in einer Versicherung zusammen, so dass die gesonderte Kinderberücksichtigung keine Änderung der Höhe der Rente gegenüber dem Fall einer reinen Beitragsorientierung zur Folge hätte.

Eine im Umlageverfahren organisierte Rentenversicherung bestreitet die Rentenzahlungen an die Altengeneration unmittelbar aus den Beitragsleistungen der Mitglieder der Aktivgeneration. Wegen der daraus resultierenden konstitutiven Bedeutung der Kindererziehung erscheint hier eine gesonderte Berücksichtigung des Kinderhabens bei der Altersrente als ,nicht-versicherungsfremde Leistung' angemessen. Eine entsprechende Orientierung findet sich in der ,Rentenentscheidung' des Bundesverfassungsgerichts vom 07.07.1992, in der betont wird, die Kindererziehung stelle einen eigenständigen Beitrag zum Erhalt der Rentenversicherung dar und sei somit bei der Rentengewährung zu berücksichtigen. ${ }^{44}$ Der VERBAND DEUTSCHER RENTENVERSICHERUNGSTRÄGER (VDR) hingegen sieht in Rentenzahlungen wegen erfolgender Kinderberücksichtigung eine versicherungsfremde Leistung, für die er einen gesonderten Zuschuss aus dem Staatshaushalt fordert. ${ }^{45,46}$ Im Jahre 1999 wurde der Bundeszuschuss für die Kinderberücksichtigung in der GRV entscheidend ausgeweitet - vgl. die Darstellung in Abschnitt 5.2 -, so dass inzwischen von einer politisch weitgehend akzeptierten (faktischen) Einordnung der rentensteigernden Berücksichtigung von Kindern als ,versicherungsfremder Leistung' auszugehen ist. ${ }^{47,48}$

$44 \mathrm{Vgl}$. Abschnitt 5.2 sowie den zugehörigen Anhang 5 (7.5).

45 Der VDR gibt für 1995 den Wert der ,versicherungsfremden Leistungen' mit ca. DM 102 Mrd. (ca. 34\% des gesamten Rentenvolumens) an, von denen ca. DM 42,7 Mrd. nicht durch den Bundeszuschuss gedeckt gewesen seien (vgl. VERBAND DEUTSCHER RENTENVERSICHERUNGSTRÄGER (1997), S. 9). Die Summe der kinderbezogenen versicherungsfremden Leistungen für 1995 (Gesamtdeutschland) beträgt ca. DM 6,4 Mrd., und macht damit ca. 6,3 v.H. der versicherungsfremden Leistungen in der Abgrenzung des VDR aus (vgl. ebd., S. 10 - eigene Berechnung).

Wobei insgesamt zu beachten ist, dass der VDR einen Anreiz hat, den Begriff der ,versicherungsfremden Leistungen' eher großzügig zu fassen, um staatliche Zuschüsse zu rechtfertigen (und somit Finanzierungslasten auf die Gemeinschaft der Steuerzahler zu ,verschieben').

Die entsprechende Einordnung der Berücksichtigung der Kindererziehung bietet eine Begründung für einen umfangreichen staatlichen Zuschuss zur GRV, der wiederum bei der Verwirklichung des Ziels einer Stabilisierung des Beitragssatzes zur GRV bei gleichzeitig ungekürzten Renten hilft. Jedenfalls wird in der Darstellung des Problems, das einen ,Entwurf eines Gesetzes zu Korrekturen in der Sozialversicherung und zur Sicherung der Arbeitnehmerrechte' der Fraktionen SPD und BÜNDNIS 90 / DIE GRÜNEN (DEUTSCHER BUNDESTAG Drucksache 


\subsection{Auswirkungen einer Rentenversicherung auf die Entwicklung der Geburtenzahlen}

Eine bedeutende Frage ist, ob von der Existenz und der konkreten Ausgestaltung einer Rentenversicherung Wirkungen auf das generative Verhalten ausgehen. Im Folgenden sollen einige Indizien für eine solche Beeinflussung des generativen Verhaltens angesprochen werden. Hierbei wird zuerst zwischen Existenz- und Ausgestaltungseffekten unterschieden, um dann auf Hinweise, die auf eine Beeinflussung des generativen Verhaltens durch eine Rentenversicherung hindeuten, einzugehen.

\subsubsection{Existenzeffekte eines Rentenversicherungssystems}

Ein wesentlicher potenzieller Grund für Auswirkungen eines Rentenversicherungssystems auf das generative Verhalten liegt in seiner Existenz begründet: Erst das Vorhandensein eines solchen Alterssicherungssystems (oder aber eines funktionierenden Kapitalmarkts) ermöglicht es dem Einzelnen, Kaufkraft (in nennenswertem Umfang) quasi ,eigenständig' von seiner Aktivphase in seine Altersphase zu transferieren (und eine entsprechende Absicherung für den Fall vorzeitiger Erwerbsunfähigkeit zu erhalten). Somit verringert die Existenz einer Rentenversicherung die individuelle Abhängigkeit von eigenen Kindern erheblich: Wer kinderlos ist, der ist im Alter nicht auf das Wohlwollen anderer Leute angewiesen, sondern hat einen (vergleichsweise sicheren) Anspruch auf Alterseinkünfte. ${ }^{49}$

14 / 45 vom 17.11.1998) begründet, auf die Notwendigkeit einer Absenkung der hohen Lohnnebenkosten hingewiesen, nicht aber auf die ,systematische Angemessenheit' eines solchen Bundeszuschusses. Die Bundesfinanzierung der Kindererziehungszeiten in der GRV mag mithin Mittel zu einem übergeordneten Zweck, nämlich der Gewährung einer Finanzhilfe zur Stabilisierung des Beitragssatzes, sein.

48 Für Einordnungen der Ausgaben der GRV zur finanziellen Anerkennung der Kindererziehung als ,versicherungsfremd' vgl. BERTHOLD und THODE (1996), HOFMANN (1996), SCHMÄHL (1997), S. 423. SCHLENGER (1998, S. 26 - 29) hingegen tendiert dazu, Leistungen der Rentenversicherung für die Kindererziehung - infolge der Organisation im Umlageverfahren - als versicherungskonform einzustufen

49 Vgl. Cigno (1991), S. 143 - 153. RoSATI (1996) präsentiert ein Modell, in dem Eltern mehr als ein Kind haben, gerade um das Risiko zu verringern, dass ein Kind als späterer ,Unterstützer' der dann alten Eltern ausfällt - entweder, weil es sich dem sozialen Druck entzieht, oder aber, was ROSATI als wichtiger ansieht, weil es infolge geringen Einkommens zu einer solchen Unterstützung nicht in der Lage ist. In diesem Fall bricht bei einem Kind die Altersversorgung völlig zusammen. Bei einer derartigen portfoliotheoretischen Begrundung für das Kinderhaben im Allgemeinen und die Bestimmung der individuell ,optimalen Kinderzahl' im Besonderen kann es nicht verwundern, dass die Existenz einer zwangsweisen Alterssicherung, die einen 
Fraglos führt die Existenz einer Rentenversicherung, die eine anonyme intertemporale Kaufkraftübertragung - zu nicht prohibitiv hohen Kosten - ermöglicht, zu einer Verringerung der Alterssicherungsbedeutung eigener Kinder. Es ist von daher zu vermuten, dass die Existenz einer Rentenversicherung mit einer im Durchschnitt niedrigeren Kinderzahl der Versicherten einhergeht. Hierbei ist allerdings zu beachten, dass eine Interdependenz zwischen der durchschnittlichen Kinderzahl (sowie der Variation dieser Zahl zwischen den Mitgliedern der Aktivgeneration) einerseits und der Existenz einer Rentenversicherung andererseits besteht: Wegen der geschilderten Probleme einer intertemporalen Kaufkraftübertragung für Kinderlose ist davon auszugehen, dass der gesellschaftliche resp. politische ,Druck' hin zur Einführung einer Rentenversicherung mit abnehmender durchschnittlicher Kinderzahl steigt. Aber nicht nur individuelle Kinderlosigkeit gefährdet bei individueller Alterssicherung den Lebensstandard im Alter: Entsprechendes gilt auch für eine vergleichsweise niedrige Kinderzahl, die es den Kindern in ihrer Aktivphase u.U. erheblich erschwert, ihre (Schwieger-)Eltern in deren Altersphase finanziell zu unterstützen. Mit abnehmender durchschnittlicher Kinderzahl nimmt die Wahrscheinlichkeit zu, dass die Kinder zu einer Versorgung ihrer Eltern nicht in der Lage sind - insbesondere auch bei besonderer Langlebigkeit der Eltern oder niedrigem Einkommen der Kinder resp. frühem Tod. ${ }^{50}$

\subsubsection{Ausgestaltungseffekte der Rentenversicherung}

In der gerade erfolgten Betrachtung wurde auf den Umstand Bezug genommen, dass erst die Existenz eines Rentenversicherungssystems die Notwendigkeit, eine größere Zahl eigener Kinder zu haben, um im Alter eine ausreichende Versorgung gewährleistet zu wissen, entfallen lässt, und somit aus Sicht des Einzelnen zu einem partiellen (materiellen) Bedeutungsverlust eigener Investitionen in das $\mathrm{Hu}-$ mankapital führt. ${ }^{51}$ Wie stark dieser Effekt ist, hängt u.a. von der konkreten Aus-

Teil der individuellen Absicherung auf das Kollektiv aller Mitglieder der Nachfolgegeneration überträgt, und somit die Bedeutung eigener Kinder als Garanten einer sicheren Einkommensversorgung im Alter verringert, in ROSATIs Modell ab einer ausreichend hohen Risikoaversion der Eltern zu einer Verringerung der durchschnittlichen Kinderzahl fuhrt (vgl. ebd., S. 291).

Eine geringe durchschnittliche Kinderzahl verringert allerdings auch die Sicherheit (und das Vertrauen in die ,Zukunftsfâhigkeit') des Alterssicherungssystems.

51 Das Wissen um die Bedeutung einer nachfolgenden Generation kann in einer rein innerfamiliär (oder in Kleingruppen) organisierten Alterssicherung nicht verloren gehen (vgl. FEUCHT (1995), S. 225): Wer die Alten nicht pflegt, gibt seinen Kindern ein schlechtes Vorbild - was passiert, wenn sich diese spăter ebenso verhalten werden? Außerdem fällt eine völlige Vernachlässigung der Eltern - noch dazu, wenn diese sich in ,Sichtweite' befinden, und es eine gesellschaftliche Erwartungshaltung hin in Richtung Altenbetreuung gibt - moralisch schwer. 
gestaltung der Rentenversicherung ab: Je leichter es ist, das Alterseinkommen ohne eigene Kinder zu sichern, desto geringer ist der (diesbezügliche) materielle Anreiz zum Kinderhaben. Somit ist bedeutsam, inwieweit innerhalb des Rentenversicherungssystems ein Rentenanspruch auch durch ,generative Leistungen' erlangt werden kann, und wie ,schwer' eine Substitution von ,Beitragszahlungen' durch ,generative Leistungen' zur Erlangung eines gegebenen Rentenniveaus ist.

\subsubsection{Empirische Befunde zu den generativen Wirkungen von Rentenversicherungen}

Für Deutschland lässt sich eine Parallelität von Einführung und sukzessivem Ausbau eines Rentenversicherungssystems einerseits und einem Rückgang der durchschnittlichen Kinderzahl feststellen. Damit ist aber kein Nachweis verbunden, dass die Existenz (und Ausgestaltung) der Rentenversicherung ursächlich hierfür ist. Insbesondere gab es zur selben Zeit eine Vielzahl weiterer Entwicklungen, die schon für sich genommen einen Rückgang der Geburtenrate erwarten lassen (Industrialisierung, Verstädterung etc.), ${ }^{52}$ die aber durch Existenz und Ausgestaltung

Wer aber keine Kinder hat, der kann nicht sicher sein, selber im Alter versorgt zu werden. Für das Umlageverfahren folgert FEUCHT aus der traditionellen innerfamiliären Alterssicherung: „Entsprechend ... kann argumentiert werden, daß außerhalb des Erwerbstätigenalters i.d.R. zwei inaktive Lebensphasen bestehen, für die im Sinne einer intertemporalen Äquivalenz von der davon profitierenden Person in der Erwerbsphase Mittel erwirtschaftet werden müssen. Wird auf Kinder verzichtet, so erfolgt die Einsparung von Aufwendungen für eine inaktive Lebensphase. Nach dieser Argumentation ist der Beitrag an die umlagefinanzierte Alterssicherung durch Kinderlose allein nicht ausreichend" (ebd., S. 225). Die Betonung der Bedeutung der Kindererziehung als Beitrag zur Sicherung der Rentenversicherung ist allerdings wesentlich älter: vgl. SCHREIBER (1955), sowie auch NELL-BREUNINGs (1981) Würdigung des Ansatzes SCHREIBERs, aber auch der Probleme der deutschen Rentenversicherung, die die kinderbezogene Seite des SCHREIBER-Ansatzes nicht berucksichtige. (Zu SCHREIBER ist allerdings anzumerken, dass er die Zahlungen an Kinder - bzw. stellvertretend an ihre Eltern - eher als Maßnahme der intertemporalen Kaufkraftumschichtung ansah (bzw. zur Verhinderung von Armut von Eltern), denn als Methode zur Beeinflussung generativer Verhaltensweisen. In einem gewissen Sinne stand er damit dem schon erwăhnten Diktum ADENAUERs, Kinder hätten die Leute immer, recht nahe: Er wollte dabei aber ,Armut‘ von Familien verhindern.)

52 Zur Tatsache, dass die Kinderzahl auf dem Lande über der in Städten liegt, vgl. (für Österreich) HOLZER und MÜNZ (1996), S. 77, Tab. 4 und für Deutschland KEMPER (1982), S. 231, Tab. 3. KLEIN und LENGSFELD (1985) können mit ,sozialstrukturellen Ursachen', d.h. durch eine Änderung der Beschäftigungs- und Sozialstruktur hin zu Berufen, die eher durch eine geringere Kinderzahl gekennzeichnet sind, ca. ein Viertel des Geburtenrückgangs von 1972 bis 1982 unter der Annahme unveränderter Alters- und Ehedauerstruktur erklären. Hierbei ist insbesondere der Bedeutungsverlust der Landwirtschaft zu nennen: In einer Untersuchung für die in den Jahren 1961 - 1965 geschlossenen Ehen erhält SCHWARZ (1977, S. 10) nur für Landwirte und mit leichten Einschränkungen - für gut verdienende Arbeiter als Haushaltsvorstand eine Kin- 
der Rentenversicherung gefördert wurden. Ferner wurde die Ausgestaltung der Rentenversicherung seit ihrer Einführung häufig modifiziert (wenn auch an einer weitgehenden ,Beitragsorientierung' festgehalten wurde), was den Nachweis konkreter Ausgestaltungseffekte weiter erschwert.

Eine Studie, die für Deutschland untersucht, welchen Anteil des Rückgangs der Geburtenrate durch Existenz und Ausgestaltung der Rentenversicherung erklärt werden kann, ist dem Verfasser nicht bekannt. Die dargestellten Probleme, die konkrete Ausgestaltung der Rentenversicherung in Variablen abzubilden, sowie die Endogenität der Ausgestaltung, d.h. ihre Abhängigkeit von den sich ändernden Umgebungsparametern, ${ }^{53}$ haben zur Folge, dass eine entsprechende Untersuchung innerhalb der vorliegenden Arbeit nicht geleistet werden kann.

Für eine Gruppe ausgewählter ,reicher' und ,armer' Länder kommen EHRLICH und ZHONG (1998) zu dem Ergebnis, dass der Anteil der Rentenversicherungseinkommen am Bruttosozialprodukt (BSP) eines Landes einen die durchschnittliche Zahl der Kinder der Versicherten verringernden Einfluss hat, wobei der Effekt in der Gruppe der ,armen' Länder deutlich ausgeprägter ist. ${ }^{54}$ Parallel dazu konstatieren sie, dass ein höherer Anteil der Rentenversicherungseinkommen am BSP die Heiratswahrscheinlichkeit verringert und die Scheidungswahrscheinlichkeit erhöht - beides Sachverhalte, die zur Erklärung eines Rückgangs der Geburtenrate

derzahl, die mit einem stabilen Generationenumfang vereinbar ist. Für alle anderen Gruppen wird die bestandserhaltende Kinderzahl - z.T. erheblich - unterschritten. Für eine - das erste Ergebnis weitgehend bestätigende - Anschlussuntersuchung vgl. SCHWARZ (1982). Vgl. auch SCHWARZ (1992a), S. 147, Tab. 4.

EHRLICH und ZHONG (1998) weisen auf die Endogenität des Anteils der Rentenversicherungseinkommen am Bruttosozialprodukt bei einer Schätzung der Auswirkung eines (umlagefinanzierten und ,sicheren') Rentenversicherungssystems auf die Fertilität hin. Eine entsprechende Endogenität der Ausgestaltung erscheint dementsprechend wahrscheinlich (Sie ist zumindest theoretisch begründbar.).

Die Autoren geben keine Ursache für den (betragsmäßig) geringeren Einfluss des Rentenversicherungssystems in den ,reicheren' Ländern an. Da sie aber nur Daten aus den Jahren 1960 bis 1989 nutzen (EHRLICH und ZHONG (1998), S. 152) erscheint es plausibel, dass in den ,reicheren' Ländern zumindest die Existenzeffekte einer Rentenversicherung auf die Kinderzahl in allen Jahren enthalten sind, also keinen Beitrag zur Erklärung eines Rückgangs der Geburtenziffer leisten kőnnen, wăhrend in den ärmeren Lăndern noch Nachwirkungen der Einführung eines (bedeutsamen und verlässlichen) institutionalisierten Alterssicherungssystems enthalten sind: Die Kinderzahl dort mag also auch sinken, weil im Zeitablauf das Vertrauen der Bevölkerung in die Rentenzusage zunimmt - die Regression erfasst aber nur die erfolgten Zahlungen, nicht die (Änderung der) erwarteten Zahlungen. 
beitragen können, aber partiell auch Folge einer niedrigeren durchschnittlichen Kinderzahl sein mögen. ${ }^{55}$

\subsection{Rentenversicherung im Kapitaldeckungsverfahren und Kinderberücksichtigung}

In der gegenwärtigen Diskussion um eine Krise des (deutschen) Rentenversicherungssystems wird regelmäßig das Kapitaldeckungsverfahren (KDV) als Referenzsystem genannt: Hier sei die ,Rendite' der Beitragszahlungen von demographischen Änderungen weitgehend unabhängig. Diese Meinung legt nahe, das Kapitel mit einer kurzen Darstellung des KDV und seiner ,Reagibilität' auf demographische Änderungen zu beschließen. ${ }^{56}$

55 Die Existenz einer Alterssicherung jenseits eigener Kinder verringert die Bedeutung der nicht marktbezogenen Institution ,Ehe' (vgl. BECKER (1981), S. 329ff. und BERTHOLD (1992), S. 524).

56 Eine andere Bedeutung des Kapitaldeckungsverfahrens als Referenzmaßstab gibt FRITZSCHE (1986): Dieser fordert, innerhalb der umlagefinanzierten Rentenversicherung ,, sollten ... nur die Vorteile ausgeglichen werden, die den Kinderlosen direkt durch das Umlageverfahren entstehen. Sie sollten demnach nach Einbeziehung des versicherungsinternen Ausgleichs nicht schlechter gestellt sein, als wenn sie aus der Rentenversicherung ausgeschlossen wären, und sich über ein Kapitaldeckungsverfahren absichern müßten. Da im Umlageverfahren unter der Annahme konstanter Beitragssătze die geleisteten Beiträge mit der Wachstumsrate des Sozialprodukts verzinst werden, besteht offenkundig ein aus dem Umlageverfahren resultierender Vorteil, wenn die Wachstumsrate den Marktzins übersteigt" (ebd., S. 203; zur involvierten Verzinsungslogik vgl. 4.3.3.2.1). Die Berücksichtigung von Kindern läuft bei FRITZSCHE für die gegenwärtigen Umgebungskonstellationen wohl darauf hinaus, dass Kinderlose im Rahmen des Umlageverfahrens einen Ausgleich für die recht geringe Verzinsung erhalten. In FRITZSCHEs Argumentation ist, ohne dass er es erwähnt, das Problem bedeutsam, welche implizite Sicht der Absicherung furr das Alter vorherrscht. Im Umlageverfahren wird in der Erwerbsphase die Erziehung durch die Eltern(generation) mit Beitragsleistungen abgegolten, und durch die Aufzucht einer nachfolgenden Generation die Basis für den eigenen Empfang von Transfers durch die Kinder(generation) während deren Altersphase gelegt. Beim Kapitaldekkungsverfahren hingegen wird eine eigenständige Absicherung für das Alter postuliert - folglich wird die Kindergeneration (im Idealfall) nicht belastet, es entsteht keine ,Dankesschuld' (vgl. FEUCHT (1995)). Die Kinderlosen leben in FRITZSCHEs Ansatz in einer finanziell für sie idealen Welt, können sie doch aus dem Umlageverfahren de facto gerade dann ausscheiden, wenn sie die Lasten für ihre Elterngeneration zu tragen hätten - ohne dass es wegen dieses Ausscheidens zu einem für das KDV typischen Verzicht auf einen Transfer von der Aktivgeneration an die Eltern(generation) kommt. Daraus folgt unter den gegenwärtigen Bedingungen eine Prämierung von Kinderlosigkeit. FRITZSCHEs Ansatz mag bei Berücksichtigung der eher überdurchschnittlichen Rendite des in Deutschland praktizierten Umlageverfahrens in der Vergangenheit (vgl. GLISMANN und HORN (1995a, 1995b, 1995c) etwas verständlicher werden, 
Grundlegendes Prinzip des KDV ist der Aufbau eines Kapitalstocks durch die Aktivgeneration und dessen sukzessive Auflösung im Alter, d.h. bei einem zur Deckung des als adäquat angesehenen Lebensstandards nicht ausreichenden Arbeitseinkommen. Es gibt im KDV (idealtypisch) keine intergenerationale Umverteilung - jede Generation ,verspeist' im Alter, was sie in ihrer Aktivphase erworben hat. Da dies wörtlich genommen nicht möglich ist, sondern sich auf den Wert des Vermögens bezieht, ergeben sich aber durchaus intergenerationale Verflechtungen: Um es zu ermöglichen, aus der Auflösung des Kapitalstocks heraus den Lebensunterhalt zu bestreiten, muss es für die angebotenen Kapitalgüter Käufer geben. Hierfür kommen (in der üblichen ökonomischen Weltsicht) nur Leute in Frage, die Kaufkraft in eine spätere Periode transferieren wollen, also primär Mitglieder einer nachfolgenden Generation. Dadurch, dass auch diese Generation ihre eigene Alterssicherung mittels eines Kapitalgüterkaufs in ihrer Aktivphase sichern will, ist Nachfrage nach dem Angebot der ,Alten' gesichert.

Der beschriebene Aktivtausch ginge idealerweise freiwillig vonstatten, würde also von beiden Seiten - sowohl von verkaufenden Alten als auch von kaufenden Jungen - als vorteilhaft angesehen. An dem gerade beschriebenen Mechanismus ändert sich auch dann nichts, wenn eine individuelle Alterssicherung durch Ankauf von Aktiva ersetzt wird durch eine Rentenzwangsversicherung nach dem Kapitaldeckungsverfahren. ${ }^{57} \mathrm{Da}$ zu jedem Augenblick der Barwert der Rentenansprüche definitionsgemäß gerade dem Wert des akkumulierten Vermögens entspricht, könnte man geneigt sein, daraus auf eine Unabhängigkeit einer derart ausgestalteten RV von den generativen Entscheidungen der Mitglieder der Aktivgeneration zu schließen. Diese Schlussfolgerung ist aber zumindest in zweierlei Hinsicht problematisch, wobei der erste Kritikpunkt der mit Abstand bedeutendere ist:

Wie schon betont, kann die Nachfrage nach den Kapitalgütern der Mitglieder der Altengeneration im wesentlichen nur von Mitgliedern einer nachfolgenden Gene-

doch systematische Probleme bleiben auch dann. (Nebenbei sei angemerkt, dass FRITZSCHE übersieht, dass zwischen der Verzinsung bei individueller Anlage am Kapitalmarkt einerseits und der bei kollektiver ,Flucht' aus dem Umlageverfahren zu erzielenden Rendite (infolge eines steigendem Kapitalangebots) eine systematische Beziehung zumindest nicht à priori ausgeschlossen werden kann. Es dürfe wesentlich attraktiver sein, als Einzelner aus einer umlagefinanzierten Rentenversicherung auszuscheiden und sich privat einer nach dem KDV organisierten Rentenversicherung anzuschließen, als Mitglied eines allgemeinen kapitalgedeckten Rentenversicherungssystems zu sein.)

Wobei als Folge des Zwangscharakters eine Abweichung der institutionell erzwungenen Höhe der Altersvorsorge von dem individuell gewünschten Volumen möglich ist. 
ration ausgehen. ${ }^{58}$ Zur ,Implosion' einer RV - sowohl bei einer Ausgestaltung im Umlage- als auch im Kapitaldeckungsverfahren - genügt, dass die Individuen um die Endlichkeit der Funktionsfähigkeit des Systems wissen (oder zu wissen meinen). Gibt es eine letzte Generation, so kann diese durch Kapitalakkumulation keine Kaufkraft in ihre Altersphase übertragen - ein wesentliches Motiv, Kapitalvermögen in der Aktivphase aufzukaufen, entfällt, und somit auch die ,Verkaufschancen' der vorhergehenden Generation. Durch rückwärtige Induktion wäre dann zu erklären, dass das System selbst für die erste Generation nicht mehr funktioniert. ${ }^{59}$

Auch die Existenz einer nachfolgenden Generation reicht also nicht aus: Die Kaufkraftübertragung von der Aktiv- in die Rentenphase ist in bedeutendem Maße vom Wiederverkaufswert der Aktiva abhängig. Da sich der Preis der Kapitalgüter auf einem Markt bildet, auf dem - vereinfacht gesagt - die Alten anbieten und die Jungen nachfragen, ist - bei konstantem Wohlstand und unverändertem Ausmaß der Gegenwartsvorliebe - davon auszugehen, dass Änderungen in der Generationenstärke zu Änderungen im Verhältnis von Kapitalgüterangebot und Kapitalgüternachfrage führen. Ein sinkender Generationenumfang bedeutet tendenziell, dass die kumulierte Nachfrage der Jungen nach Kapitalgütern sinkt. Auf einem stabilen Markt führt aber ein Überschussangebot zu einer Preissenkung - der Kaufkrafttransfer in die Altersphase verteuert sich, d.h. für ein gegebenes Kaufkraftvolumen im Alter muss in der Jugend mehr gespart werden. Insoweit besteht also

58 Was auch intuitiv einleuchtet: (Konsum-)Kaufkraft bedeutet in letzter Konsequenz Anspruch auf einen Teil der Produktion Dritter. Gibt es keine Aktivgeneration mehr, kommt also die Produktion zum Erliegen, so kann Geld keine Kaufkraft mehr vermitteln. Man muss dann selber das produzieren, was man konsumieren möchte. Dieser elementare Sachverhalt ist nicht von der gewählten Form der Alterssicherung (UV oder KDV, freiwillig oder zwangsweise, privat oder institutionalisiert) abhängig.

59 Selbstverständlich liegen in der Realität weniger eindeutige Indizien vor: So ,nur' ein abnehmender Umfang der Aktivgeneration, womit das ,Ende' der Funktionsfähigkeit des Rentenversicherungssystems nicht deutlich absehbar ist, dazu noch die Möglichkeit eines Handels mit dem Ausland, steigender Wohlstand, der (c.p.) eine individuell höhere Nachfrage nach Alterssicherung bewirkt etc. Ferner ist eine staatliche Rentenversicherung im KDV weiterhin mit allen Zwangsmitteln zur Beitragseintreibung ausgestattet - auf diese Art und Weise lässt sie sich durchaus auch bei abnehmendem Bevölkerungsumfang resp. geringer erwarteter Rendite der Beitragszahlungen eine Zeit lang weiterbetreiben. Werden die ,Renditeaussichten' aber allzu schlecht, so dürfte der Widerstand gegen die zwangsweise Einbeziehung in das (Rentenversicherungs-)System sukzessive zunehmen und die administrativen Kosten deutlich steigen (sowie die Suche nach ,Schlupflöchern' und die ,Flucht' aus dem System intensiviert werden). 
durchaus eine Sensitivität einer kapitalgedeckten RV gegenüber Fertilitätsänderungen..$^{60,61}$

Der zweite, eher praktische Ansatzpunkt der Berücksichtigung einer Generationenabfolge liegt in Änderungen der Restlebenserwartung bei Renteneintritt begründet. ${ }^{62}$ Steigt diese über den bei der ,Lebenseinkommensplanung' prognostizierten Wert hinaus, so reicht der Wert der zu veräußernden Aktiva nicht mehr zur Aufrechterhaltung des geplanten Alterslebensstandards aus - in Zwangssystemen ist in diesem Fall eine (zumindest) teilweise Überwälzung der entstehenden finanziellen Lasten auf die nachfolgende Generation zu erwarten. Sie setzt aber voraus, dass es eine weitere Generation gibt, die zur Tragung fähig ist. ${ }^{63}$

60 In einer Gesellschaft, bei der der Lebensstandard eines repräsentativen Mitglieds einer Generation intertemporal steigt, kommt es zu einer Abschwăchung des gerade erlăuterten Prozesses: Zwar steht weiterhin den Verkäufern eine zahlenmäßig geringere Nachfolgegeneration gegenüber, aber das einzelne Mitglied dieser Generation hat (u.U.) zur Sicherung seines Lebensstandards im Alter eine erhöhte Nachfrage nach Anlagegütern, so dass die Gesamtnachfrage nach dem ,Angebot' der Alten nicht sinken muss. Relativ zu einem größeren Umfang der Nachfragegeneration bleibt es aber bei einer Preissenkungstendenz. Diese kann nur dadurch aufgehoben werden, dass der Wohlstand - und dementsprechend die Nachfrage nach Anlagegütern der Aktivgeneration gerade deswegen so hoch ist, weil die Generation weniger Mitglieder hat und die Nachfrage nach Gütern zum Zwecke der Alterssicherung wohlstandselastisch ist. Solange ein sinkender Generationenumfang also einen steigenden Wohlstand eines typischen Mitglieds der Generation fördert, ist der Gesamteffekt auf die Nachfrage nicht ohne weiteres festzumachen.

${ }^{61}$ Außerdem ist bei einem Vergleich mit einer umlagefinanzierten RV der (einmalige) Niveaueffekt der jeweiligen Ausgestaltungsform auf den Kapitalstock einer Volkswirtschaft mit seinen Wirkungen auf das (reale) Zinsniveau zu beachten (wobei das Zinsniveau wiederum den Wert des Kapitalstocks beeinflusst). GROHMANN (1986) schätzt für Mitte der 80'er Jahre den zur Sicherung der bestehenden Rentenansprüche im Rahmen des Umlageverfahrens notwendigen Kapitalstock für Westdeutschland auf ca. DM 5 Billionen - und damit auf eine Summe, der (fast) dem damaligen Wert des reproduzierbaren Sachvermögens zu Wiederbeschaffungspreisen entspricht. Er errechnet mithin die Notwendigkeit, bei inländischer Geldanlage den Kapitalstock de facto zu verdoppeln! (Der WISSENSCHAFTLICHE BEIRAT BEIM BUNDESMINISTERIUM FÜR WIRTSCHAFT (1998) schätzt den aktuellen kumulierten Wert der Rentenansprüche im Rahmen der GRV auf ca. DM 10 Mrd.)

Vgl. EITENMÜLLER (1998), S. 3.

Weitere Extremfälle, deren praktische Bedeutung für das Festhalten Deutschlands an einer im UV organisierten RV vermutlich kaum zu uberschätzen ist, stellen Kriege, Naturkatastrophen etc. dar: Wird der Kapitalstock der Alten weitestgehend entwertet (oder gar physisch vernichtet), ist eine Sicherung des ,Überlebens' der Alten nicht ohne intergenerative Solidarität möglich. Diese kann sich dann als Zwangssolidarität im Rahmen einer umlagefinanzierten Rentenversicherung oder aber in Sozialhilfetransfers manifestieren. 
Aus dem gerade Gesagten sollte jedoch nicht geschlossen werden, dass eine im Kapitaldeckungsverfahren organisierte RV nicht besser als eine im Umlageverfahren organisierte dazu geeignet sein kann, unter den realen Bedingungen Änderungen im generativen Verhalten zu ,bewältigen'. Insbesondere die internationale Handelbarkeit von Kapitalvermögen erlaubt es einem ,kleinen' Land, die Altersversorgung seiner Bevölkerung von deren eigenen Fertilität zumindest teilweise abzukoppeln. Eine dauerhafte Lösung stellt die Einbeziehung ausländischer Staaten in die Anlagestrategie aber nur dann dar, wenn diese nicht ebenfalls deutliche Rückgänge in der Rendite (z.B. infolge vergleichbarer demographischer Änderungen) realisieren. ${ }^{64}$ Außerdem ist zu beachten, dass ausländisches Interesse an Anlagen in einem , aussterbenden Land' nur in beschränktem Umfang (d.h. nur zu günstigen Preisen) vorhanden sein dürfte. ${ }^{65}$ Die Rentensicherheit deutscher Beitragszahler primär durch Anlage von Geldern im Ausland zu sichern, erscheint aber auch nicht unproblematisch: Es existieren dann Wechselkursrisiken, aber auch die Gefahr der Enteignung ausländischer Anteilseigner bzw. generelle Verstaatlichung, sowie das ,politische' Problem, dass mit den Geldern der deutschen Aktivgeneration die Arbeitsplätze ihrer ausländischen Konkurrenten kapitalintensiv und wettbewerbsfähig ausgestattet werden (was heimische Arbeitsplätze weniger sicher macht - und damit teilweise das Einkommen gefährdet, aus dem die Alterssicherung bestritten werden soll) ${ }^{66}$ Ferner verbessert die Strategie einer höheren Pro-Kopf-Kapitalausstattung der Aktivgeneration im KDV die Möglichkeit eines zeitweisen ,Lebens von der Substanz', einer partiellen Umstellung der Produktion von Kapital- hin zu Konsumgütern, also einen zeitweisen Verzicht auf Reinvestitionen. ${ }^{67,68}$

64 Vgl. WiSSENSCHAFTLICHER BEIRAT BEIM BUNDESMINISTERIUM FÜR WIRTSCHAFT (1998), S. 12. Dieser stellt aber zur gegenwärtigen Situation am internationalen Kapitalmarkt fest: „Ein weltweiter Mangel an attraktiven Investitionsstandorten, die einen Schutz vor einer Entwertung des Kapitals durch schrumpfende Bevölkerung bieten, ist auf absehbare Zeit wahrlich nicht zu fürchten" (ebd., S. 12). Auch die Risiken einer Auslandsanlage sieht er als durch Risikostreuung beherrschbar an (vgl. ebd., S. 12), konstatiert also keine nennenswerte positive Korrelation der Anlagerisiken in verschiedenen Ländern mit ,nicht-schrumpfender Bevölkerung'.

65 Allgemeiner lässt sich festhalten, dass ein Bevölkerungsrückgang „die Verwertungsbedingungen eines gegebenen Kapitalstocks verschlechter[t]“ (WISSENSCHAFTLICHER BEIRAT ... (1998), S. 12), die Anlage mithin an Attraktivität einbüßt.

66 Es besteht allerdings die Möglichkeit, dass der Kapitalexport bei der Beschleunigung des Strukturwandels hin zu einer Dienstleistungsgesellschaft (Altenpfleger, Gerontologen, etc.) hilft - und somit aus der Sicht der Alten nützlich ist.

67 Vgl. NEUMANN (1986), S. 45ff. 
Auch wenn - wie gerade erläutert - eine im KDV ausgestaltete RV nicht ohne nachfolgende Generation, also ohne die Bereitschaft von Mitgliedern der Gesellschaft, Nachwuchs heranzuziehen, existieren kann, so ist doch die unmittelbare Bedeutung von Kindern gegenüber dem UV geringer. Die Notwendigkeit einer besonderen - über die reine Hinterbliebenenversorgung hinausgehenden - Kinderberücksichtigung ist deshalb im Rahmen einer im UV organisierten RV systembedingt größer. Insbesondere ist $\mathrm{zu}$ bedenken, dass bei einer im KDV organisierten Rentenversicherung eine in ihrem Umfang hinter der Elterngeneration zurückbleibende Aktivgeneration (c.p.) von den infolge der veränderten demographischen Situation verringerten Preisen der Anlagegüter ,profitiert' (was ihre Ausbeutung durch die Alten aber insoweit nicht ausschließt, als die Aktiven in einem institutionalisierten und mit Zwang bewehrtem Alterssicherungssystem die Jungen zu einer über deren individuellen Präferenzen - auch und gerade im Hinblick auf die von ihnen erwartete Rendite der Beitragszahlungen zur GRV - hinausgehenden Nachfrage nach Anlagegütern verpflichten können). Ein vergleichbarer partieller Rückkopplungsmechanismus existiert in einer im Umlageverfahren organisierten RV nicht - was eine Kinderberücksichtigung innerhalb dieses Rentenversicherungssystems dringlicher macht.

68 Die Ausführungen SCHREIBERs (1955, S. 89) hierzu (,Theoretisch vorstellbar wäre eine Auflösung von Volksvermögen in der Form der Unterlassung von Ersatzinvestitionen für sich abnutzendes Realkapital. Von dieser Möglichkeit Gebrauch machen hieße: die Wirtschaftsgesellschaft auf eine primitivere Stufe des Lebensstandards zurulckwerfen - eine praktisch unmögliche Forderung.") erscheinen für den Fall einer abnehmenden Bevölkerung nicht uberzeugend: Es bedarf dann eines geringeren Kapitalstocks, um eine vergleichbare Kapitalausstattung pro Beschäftigtem zu erhalten, so dass ein teilweiser Verzicht auf Reinvestitionen nicht zu einer Senkung des Lebensstandards fuhren muss. 


\section{Kinderberücksichtigung bei einer im Umlageverfahren organisierten Rentenversicherung}

Im folgenden Kapitel wird die Bedeutung einer nachfolgenden Generation in einem im Umlageverfahren (UV) ausgestalteten Rentensystem untersucht. Hierzu werden verschiedene ,reine' Formen des Umlageverfahrens vorgestellt. Wiewohl diese Versionen sicher nicht annähernd die komplexe Ausgestaltung des (gegenwärtigen) deutschen Rentenversicherungssystems wiedergeben, sind sie doch in der Lage, grundsätzliche Einsichten zu vermitteln, die über ihren stark eingeschränkten Annahmekreis hinausreichen.

\subsection{Grundlegende konzeptionelle Versionen einer umlagefinanzierten Rentenversicherung}

Nachfolgend werden unterschiedliche Ausgestaltungsmöglichkeiten eines Umlageverfahrens dargestellt. Auf diese unterschiedlichen Versionen wird im weiteren Verlauf der Arbeit noch häufig Bezug genommen.

\subsubsection{Die Grundgleichung des Umlageverfahrens einer Rentenversicherung}

Zur Herleitung der möglichen Ausgestaltungsformen eines im Umlageverfahren organisierten Rentenversicherungssystems wird zuerst die ,charakteristische Gleichung' dieses Alterssicherungssystems betrachtet. Sie lautet:

$\sum_{i=1}^{N_{t}} b_{t}^{i}=\sum_{j=1}^{N_{t-1}} r_{t}^{j}$

\section{Gleichung 3-1}

(mit $N_{t}$ : Zahl der Beitragszahler in Periode $t ; i(j)$ : Index der Beitragszahler (Rentenbezieher); $b_{t}^{i}$ : Rentenversicherungsbeitrag des in Periode $t$ Beitragszahlenden $i ; N_{t-1}$ : Zahl der Rentner in Periode $t$ (Zahl der Beitragszahler in $t-1$ ); $r_{t}^{j}$ : Rentenzahlung an $j$ in Periode $t$ ). 
Diese ,charakteristische Gleichung' bringt das definitorische Kennzeichen eines Umlageverfahrens zum Ausdruck, nach dem die Summe der Beitragseinnahmen in jeder Periode der Summe der Rentenzahlungen entsprechen muss. ${ }^{69}$

\subsubsection{Vier Versionen eines Umlageverfahrens im Überblick}

Bei gegebener Zahl von Beitragszahlern und Rentnern bieten sich vier verschiedene Ausgestaltungsformen an, die sich durch unterschiedliche Festlegung der relevanten Rentenversicherungsparameter unterscheiden. Diese sollen nun kurz charakterisiert werden - eine ausführlichere Analyse der einzelnen Versionen, bei der dann auch auf die Bedeutung von Kindern und deren mögliche Berücksichtigung innerhalb der einzelnen Ausgestaltungen eingegangen wird, erfolgt später (vgl. 3.2 und 3.3).

Version 1: $\quad$ Exogene Festlegung des Beitragsniveaus in der Form einer Fixierung der durchschnittlichen Absolutbeiträge der Mitglieder der Aktivgeneration.

Durch diese Festlegung ist die Beitragssumme vorgegeben, und somit auch die Summe der Rentenzahlungen an die Mitglieder der Rentnergeneration.

Version 2: $\quad$ Exogene Festlegung des Rentenniveaus in der Form einer Fixierung der durchschnittlichen absoluten Rentenhöhe der Mitglieder der Rentnergeneration.

Durch diese Festlegung ist die Beitragssumme vorgegeben, die von den Mitgliedern der Aktivgeneration aufgebracht werden muss. Stimmen ferner die Charakteristika der Mitglieder der jeweiligen Generation überein, so sind auch die jeweiligen absoluten Rentenbeiträge gegeben.

Die beiden gerade vorgestellten Möglichkeiten der Festlegung rentenrelevanter Parameter implizieren in einer dynamischen Umgebung eine erhebliche Veränderung des relativen Lebensstandards der jeweiligen Generation. Die hierfür ursächliche Dynamik kann Folge einer Änderung des Verhältnisses der Stärke der Rentnergeneration im Vergleich zur Aktivgeneration sein, aber auch die Folge

69 Die Gleichung muss - natürlich - nur dann immer erfullt sein, wenn keine Reserven existieren, keine Verschuldung möglich ist, keine Verwaltungskosten vorliegen, die Synchronisation der Zahlungen gesichert ist, die gesamten Rentenzahlungen aus dem Beitragsaufkommen finanziert werden (keine Zuschüsse bzw. Abfuhrungen) etc. Diese Annahmen haben aber für die hier zu betrachtenden grundlegenden Zusammenhänge keine Bedeutung. 
einer im Durchschnitt veränderten Produktivität der Mitglieder der Aktivgeneration. Ohne regelmäßige ,Nachsteuerung' der Beitrags- bzw. Rentenhöhe besteht folglich die Möglichkeit einer beitragsmäßigen Überforderung der Mitglieder der Aktivgeneration (so z.B. bei abnehmendem Generationenumfang und konstanter durchschnittlicher Produktivität der Beschäftigten, sowie vorgegebener Rentenhöhe) oder einer Nicht-Partizipation der Mitglieder der Rentnergeneration an einem steigenden Lebensstandard der Aktivgeneration (so z.B. bei steigender durchschnittlicher Produktivität der Aktiven, konstantem Generationenumfang und konstanter Beitragszahlung zur RV). Die beiden nachfolgenden Ausgestaltungsformen verhindern die eine bzw. andere Möglichkeit; sie haben dafür aber wie sich im weiteren Verlauf der Arbeit noch zeigen wird - andere Nachteile.

Version 3: $\quad$ Exogene Festlegung eines intertemporal konstanten Beitragssatzes auf das (im Zeitablauf übereinstimmend abgegrenzte) rentenversicherungspflichtige Einkommen der Mitglieder der Aktivgeneration.

Hierdurch ist das Beitragsaufkommen (indirekt) vorgegeben, und bei Übereinstimmung der Charakteristika der Mitglieder einer Generation sind es auch deren absolute Rentenbeiträge bzw. -zahlungen.

Version 4: $\quad$ Exogene Festlegung des relativen Lebensstandards eines repräsentativen Mitglieds der Rentnergeneration verglichen mit einem repräsentativen Mitglied der Aktivgeneration.

Bei gegebenen Mitgliederzahlen der jeweiligen Generationen und gegebenem Einkommen der Mitglieder der Aktivgeneration ist die an die Rentner zu übertragende Beitragssumme festgelegt, und somit auch der Beitragssatz für die Mitglieder der Aktivgeneration.

\subsection{Versionen mit Fixierung von Beiträgen oder Renten (Versionen 1 und 2)}

Nachdem im vorangegangenen Abschnitt vier verschiedene Grundtypen einer im Umlageverfahren organisierten Rentenversicherung vorgestellt worden sind, sollen im Folgenden die beiden zuerst charakterisierten Ansätze (Versionen 1 und 2) genauer dargestellt und hinsichtlich der Bedeutung von Kindern und der Möglichkeit ihrer Berücksichtigung untersucht werden. Die beiden Ansätze sind durch die exogene Festlegung von Absolutbeträgen (sei es auf Beitrags-, sei es auf Rentenseite) gekennzeichnet. Ausgangspunkt für die Darstellung ist in beiden Fällen die charakteristische Gleichung eines Umlageverfahrens. 


\subsubsection{Version 1 eines Umlageverfahrens unter statischen Bedingungen}

Im Folgenden wird Version 1 eines Umlageverfahrens, also die exogene Festlegung eines intertemporal konstanten durchschnittlichen (Absolut-)Beitrags zur Rentenversicherung, betrachtet.

\subsubsection{Vorbemerkungen}

Um die wesentlichen Zusammenhänge deutlich zu machen, werden eine Reihe vereinfachender Annahmen eingeführt. So leben alle Versicherten zwei ,versicherungsrelevante' Perioden lang: In der ersten Periode sind sie erwerbstätig und ziehen Kinder heran (wobei vorauszusetzen ist, dass es eine nachfolgende Generation gibt, also nicht alle Aktiven eine Kinderzahl von 0 realisieren), in der zweiten Periode sind sie Rentner. Die Lebensdauer aller stimmt überein und ist ihnen mit Sicherheit bekannt. Es besteht in allen relevanten Perioden Zwang, der RV beizutreten. Am Anfang der Aktivperiode muss jeder Aktive einmalig einen Beitrag in die RV einzahlen. Im Durchschnitt aller Beitragszahler beträgt dieser Beitrag $b_{t}$. Die nachfolgende Generation der Aktiven zahlt im Durchschnitt einen Beitrag in derselben Höhe. Im Unterschied zu den durchschnittlichen stimmen die individuellen Beitragszahlungen aber nicht unbedingt überein. Die zuvor dargestellte Stromgrößengleichung einer im UV organisierten RV (vgl. Gleichung 3-1) vereinfacht sich unter den getroffenen Annahmen zu:

$N_{t} \cdot b_{t}=N_{t-1} \cdot r_{t}$

Gleichung 3-2

(mit $r_{t}$ : durchschnittliche Rentenzahlung an einen Rentner in Periode $t$ ).

\subsubsection{Zur Bedeutung von Kindern für das durchschnittliche Rentenniveau im Zeitablauf bei einer umlagefinanzierten Rentenversicherung nach Version 1}

Bei der Version 1 gibt es in der eben dargestellten Form keinen intertemporalen Zusammenhang zwischen den Beitragszahlungen der Erwerbstätigen einerseits und den von ihnen in ihrem Alter empfangenen Rentenzahlungen andererseits. Der hier vorgestellte Grundtyp kann - was allerdings auch z.B. für das in Deutschland real existierende Umlageverfahren gilt - in der Realität nur existieren, wenn die gegenwärtigen Beitragszahler darauf vertrauen, dass die Rentenversicherung auch in ihrer Altersphase fortgeführt wird und sie mit ihren heutigen Zahlungen ein ,Anrecht' auf spätere Rentenzahlungen in ,angemessener' Höhe erwerben. 
Durch eine Umformung von Gleichung 3-2 erhält man:

$$
\frac{r_{t}}{b_{t}}=\frac{N_{t}}{N_{t-1}}=1+g_{t}
$$

Gleichung 3-3

(wobei $g_{t}$ die Wachstumsrate des Umfangs der Aktivgeneration von Periode $t-1$ bis Periode $t$ darstellt) ${ }^{70}$.

Dieser Beziehung lassen sich zwei Grundsachverhalte entnehmen: ${ }^{71}$

- Die Verzinsung in einer im Umlageverfahren gestalteten Rentenversicherung bei intertemporaler Konstanz der Beitragszahlung entspricht gerade der Wachstumsrate der Zahl der in der RV versicherten Erwerbstätigen. ${ }^{72}$

- Bei exogenem $g_{t}$ gibt es für die ,Steuerung' der Rentenversicherung genau einen Freiheitsgrad. Der ,Planer' kann entweder die Beitragshöhe, oder aber die Rentenhöhe frei variieren - nicht aber beide Parameter. ${ }^{73}$

Die Version 1 verdeutlicht die positiven externen Effekte des Kinderhabens im Rahmen einer im Umlageverfahren ausgestalteten RV in pointierter Weise. Formt man die obige Gleichung um, so erhält man:

$$
r_{t}=\frac{N_{t}}{N_{t-1}} \cdot b_{t}
$$

\section{Gleichung 3-4}

Differenziert man bei intragenerational konstanter positiver Beitragsleistung die Rentenhöhe nach der Zahl der Beitragszahler, ergibt sich: $\frac{\partial r_{t}}{\partial N_{t}}=\frac{1}{N_{t-1}} \cdot b_{t}>0$.

70 Die ausgewiesenen Wachstumsrate ist also - unter realitătsnahen Bedingungen - nicht mit der Wachstumsrate der Bevölkerung zu verwechseln, in die Änderungen im Umfang der Rentnerpopulation (z.B. infolge von Änderungen in der durchschnittlichen Restlebensdauer nach Renteneintritt) mit eingehen.

71 Wobei beide Implikationen, auch wenn sie Unterschiedliches betonen, nicht voneinander zu trennen sind.

Vgl. SAMUELSON (1958), insbes. S. $471 \mathrm{f}$.

73 Im Falle der exogenen Festlegung einer absoluten Rentenhöhe befindet man sich im als Version 2 charakterisierten Regime. 
Das Hinzukommen eines weiteren Beitragszahlers in der nächsten Generation erhöht die Rente jedes einzelnen Rentners (die Beitragszahlung dieses weiteren Beitragszahlers in Höhe von $b_{t}$ wird auf die $N_{t-1}$ Rentenberechtigten aufgeteilt). Während $N_{t-1}-1$ Rentner einen Netto-Vorteil von $\frac{b_{t}}{N_{t-1}}$ durch einen weiteren Beitragszahler haben, verbleiben bei einem der Rentner die - außerhalb der RV angesiedelten - Netto-Kosten der Kindererziehung, so dass sein (finanzieller) Netto-Vorteil eines weiteren Kindes regelmäßig negativ ist. Die von der Gesellschaft realisierten positiven (pekuniären) externen Effekte innerhalb des UV betragen (unter Verzicht auf Abdiskontierung) $\frac{N_{t-1}-1}{N_{t-1}} \cdot b_{t}\left(\approx b_{t}\right.$ für $\left.N_{t-1}>>\right)$. Während in kleinen Gruppen die unmittelbare Bedeutung jedes weiteren Beitragszahlers deutlich hervortritt, wird dieser Effekt in großen Gruppen zunehmend schwächer, ${ }^{74}$ und somit kann die Illusion entstehen, die Rendite einer umlagefinanzierten RV sei unabhängig von der ,biologischen Regeneration' der Aktivbevölkerung. Die finanziellen Anreizkonstellationen sind regelmäßig derart, dass wegen fehlender Internalisierung der positiven rentenmäßigen externen Effekte eines weiteren Kindes kein Anreiz zur Ausweitung der Kinderzahl gesetzt wird, obwohl ohne ein Umlageverfahren - bei einer entsprechenden Unterhaltsverpflichtung der Kinder - ein solcher Anreiz bestünde und gesellschaftlich (ökonomisch) möglicherweise wünschenswert wäre. $(\mathrm{Ob}$ das Bestehen eines Anreizes zum Kinderhaben im Rahmen der GRV tatsächlich im finanziellen Interesse der Rentnergeneration ist, hängt jedoch von der Reagibilität des Kinderhabens auf finanzielle Anreize $\mathrm{ab}^{75}$ )

Es ist allerdings zu beachten, dass durch die Einzahlungen in das UV - wenn nicht systemimmanent, so doch in jeder praktizierten Ausgestaltung - ein Anspruch auf den Erhalt von Rentenzahlungen im Alter begründet wird (ohne dass hier näher auf deren Höhe eingegangen werden soll). Wer Kinder hat, die in die RV einzahlen, der zieht eben auch spätere Rentenbezieher heran. Während der Wert der Kinder für die Altersgenossen ihrer Eltern positiv ist, gilt dies für Ange-

$74 \frac{\partial^{2} r_{t}}{\partial N_{t} \partial N_{t-1}}=-\frac{1}{\left(N_{t-1}\right)^{2}} \cdot b_{t}<0$ - dies ändert aber nichts daran, dass die ,Rentnergeneration' in toto pro zusätzlichem Beitragszahler (Kind) einen ,Netto-Rentengewinn' von $b_{t}$ realisiert.

Insbesondere sind die Kosten einer Kinderberücksichtigung mit gesteigerten Beitragseinnahmen infolge höherer Kinderzahlen zu vergleichen. (In einem erweiterten Modellrahmen ist auch die Reagibilität der Summe der rentenversicherungspflichtigen Einkommen auf eventuelle Änderungen der Fertilität zu beachten.) 
hörige der eigenen Generation der Kinder nicht unbedingt: Ist die Anzahl der Kinder unterdurchschnittlich, partizipieren sie aber durchschnittlich an den umgelegten Beträgen, so führen die Kinder zu einer Senkung der Rendite der Umlageversicherung für die Mitglieder der eigenen Generation. Hieraus ergibt sich das Problem, eine Abschätzung für den ,kumulierten rentenmäßigen Wert' der nachfolgenden Generationen zu finden, um die ,Schädigung' der RV (bzw. der in ihr Versicherten) durch ,fehlende Beitragszahler' angeben zu können. Dabei ist zu berücksichtigen, dass fehlende Beitragszahler eine Generation später auch keine Renten beziehen.

Zur weiteren Behandlung erfolgt die Annahme, alle nachfolgenden Generationen eines ,fehlenden Beitragszahlers' hätten sich - generativ - wie die anderen Versicherten ihrer Generation verhalten.

Als Begründung hierfür kann darauf verwiesen werden, dass keine anderen Informationen vorliegen. Somit ist nach dem ,Prinzip des unzureichenden Grundes' eine Abweichung vom Durchschnitt nicht zu unterstellen. Zum anderen lässt sich bei unterschiedlichem ,rentenmäßigem Wert' einzelner Kinder der ,Wert' fehlender Kinder nie berechnen, da ein unendlicher Regress vorliegt. ${ }^{76}$ Vor diesem Hintergrund wird für die ,fehlenden' Kinder ein jeweils durchschnittliches generatives Verhalten unterstellt. ${ }^{77}$

Unter der getroffenen Annahme folgt, dass die ,fehlenden' Kinder (verglichen mit anderen Kindern ihrer Generation) für die RV weder einen besonderen, Wert' darstellen, noch besondere ,Belastungen' induzieren. Ihr Hinzukommen bzw. Fernbleiben ändert an den Pro-Kopf-Werten der nachfolgenden Generationen nichts. Der gesamte Netto-Effekt eines ,fehlenden Kindes' manifestiert sich in seiner fehlenden Beitragszahlung zu Beginn der Aktivperiode - und ist somit problemlos abschätzbar.

\subsubsection{Möglichkeiten der Kinderberücksichtigung}

Im Folgenden werden - für das oben beschriebene Grundmodell - zwei unterschiedliche Möglichkeiten untersucht, wie sich die individuellen finanziellen Anreize zum Kinderhaben besser mit den ,gesellschaftlichen Vorteilen' des Kinder-

76 Wenn die RV unendlich besteht, so fehlen abzählbar unendlich viele Kinder infolge eines einzelnen in der ersten Periode ,fehlenden' Kindes - und für alle diese Kinder müsste ein ,rentenmäßiger Wert' berechnet werden.

77 Für eine vergleichbare Argumentation - basierend auf einer formalen Abschätzung - vgl. SINN (1997), S. 11 - 14. 
habens (für die RV) in Übereinstimmung bringen lassen: Zum einen eine Beitragsstaffelung nach der Kinderzahl (bei gegebener Rentenhöhe), zum anderen eine Abhängigkeit der späteren Rentenhöhe von der Kinderzahl (bei gegebenem Beitrag in der Aktivzeit).

In beiden Fällen wird jeweils ein einzelner Erwachsener mit seinen Kindern betrachtet. Eine gesonderte Berücksichtigung der Tatsache, dass jedes Kind zwei Elternteile hat, und dementsprechend die Relaxation der Annahme einer ganzzahligen Kinderzahl pro Versicherten, ist möglich, führt aber nicht zu neuen Erkenntnissen, die die dann notwendigerweise kompliziertere Darstellung rechtfertigen können.

\subsection{Beitragsstaffelung nach Kinderzahl}

Im Folgenden soll zuerst der Ansatz einer Beitragsstaffelung nach der Kinderzahl vorgestellt und seine möglichen Implikationen skizziert werden, um dann zu einer formalen Darstellung des Ansatzes überzugehen. Letztere wird ergänzt um einige Beispielrechnungen.

\subsection{Eigenschaften einer Beitragsstaffelung nach Kinderzahl}

Eine Beitragsstaffelung nach Kinderzahl liegt vor, wenn die vom Versicherten zur Begründung eines gegebenen Altersrentenanspruchs zu leistende Beitragszahlung mit zunehmender Kinderzahl abnimmt. Aus Vereinfachungsgründen wird angenommen, dass durch diese Beitragsstaffelung der durchschnittliche Beitrag eines Mitglieds der Aktivgeneration nicht verändert wird, so dass die zur Verteilung an die Rentnergeneration zur Verfügung stehende Beitragssumme unverändert bleibt. Vielleicht lässt sich diese Annahme auch damit begründen, dass - wie bereits aufgezeigt (vgl. 3.2.1.2) - bei gegebenem Beitrag Kinder nicht-reziproke positive externe Effekte (im Rahmen der GRV) induzieren. Diese positiven externen Effekte kommen aber (ausschließlich) den Mitgliedern der gegenwärtigen Aktivgeneration in ihrer Rentenphase zugute, so dass von daher die Konstanz der Rentenzahlungen an die gegenwärtige Rentnerschaft angemessen ist.

Eine solche Regelung ändert (selbstverständlich) nichts daran, dass für die Existenz eines umlagefinanzierten Rentenversicherungssystems die Aufzucht einer nachfolgenden Generation, die ihrerseits durch ihre Beiträge die Renten der dann Alten finanziert (und gleichzeitig eine weitere nachfolgende Generation aufzieht), notwendig ist. Sie erlaubt aber - von der Idee her - einen Ausgleich der individuellen finanziellen Vorteile der Kinderlosigkeit. Bei der Festlegung des Ausmaßes des Ausgleichs gibt es allerdings Gründe, die gegen einen vollständigen Aus- 
gleich sprechen: So hat die Existenz einer institutionalisierten Rentenversicherung im Umlageverfahren auch für Eltern gewisse Vorteile. Insbesondere wird von der Familie der Druck genommen, intrafamiliär um Transfers von den Kindern an die Eltern in deren Altersphase zu verhandeln; die Alten haben eine Rechtsposition gegenüber Mitgliedern der nachfolgenden Generation, benötigen also kein ,Pfand' (wie beim Tausch ,Bauernhof' gegen ,Pflegezusage' durch den ältesten Sohn); außerdem entfallen mit intrafamiliären Verhandlungen möglicherweise verbundene atmosphärische Belastungen. Es kommt mithin zu einer - allokativ positiv zu wertenden - Verringerung von Transaktionskosten infolge einer normierten Ausgestaltung. Ferner erfolgt eine Absicherung der Eltern für den Fall erheblich unterdurchschnittlichen Einkommens der Kinder, also geringerer Möglichkeit dieser, die Eltern zu unterstützen. ${ }^{78}$ Die institutionalisierte Organisation der Absicherung hat somit auch für Leute mit überdurchschnittlicher Kinderzahl einen gewissen Servicecharakter, und außerdem Elemente einer Versicherung. Insoweit erscheint es angemessen, Teile der mit der Einführung einer institutionalisierten Alterssicherung einhergehenden Belastungen Kinderhabender als ,Preis' für verschiedene Leistungen der RV anzusehen; der Umstand, dass die teilweise Sozialisierung der Erträge des Kinderhabens Anreize zugunsten einer verringerten Investition der Eltern in Humankapital (quantitativ und qualitativ) setzt, reicht per se also nicht aus, die Existenz einer im Umlageverfahren ausgestalteten Rentenversicherung allokativ (unter Berücksichtigung von Transaktionskosten) abzulehnen, ${ }^{79}$ oder einen vollständigen Ausgleich der kinderbezogenen externen Effekte (ohne Berücksichtigung der auch intern anfallenden Vorteile) zu fordern.

Aus der Ablehnung der Notwendigkeit eines vollständigen Ausgleichs der externen Effekte des Kinderhabens im Rahmen einer umlagefinanzierten RV folgt aber nicht die entgegengesetzte Extremposition eines Verzichts auf jedwede Kinderberücksichtigung, wie er von HASE vertreten wird: Dieser kritisiert, durch die Forderung nach Berücksichtigung der Kindererziehung werde „der heute bereits in vielen Bereichen virulente Gegensatz zwischen Familien und Singles, Kindererziehenden und Kinderlosen in ein seit seiner Entstehung überaus breit angelegtes ... Sicherungssystem hineingetragen, das diesen Gegensatz bisher überaus erfolgreich ,neutralisieren' konnte ${ }^{680}$. Die institutionalisierten Systeme der Alterssicherung hätten doch gerade die Absicherung unabhängig von der eigenen Kinderzahl zum Ziel. ${ }^{81}$ HASE unterscheidet bei seiner Argumentation nicht zwischen ei-

78 Entsprechendes gilt auch furr den Fall, dass die Kinder früher als ihre Eltern sterben.

79

80

81

Vgl. HASE (1992), S. 527.
Vgl. SINN (1998).

HASE (1992), S. 526. 
ner (unumstritten sinnvollen) Altersabsicherung auch Kinderloser einerseits, und den Konditionen einer solchen Absicherung andererseits. Außerdem kann eine Ausgestaltungsform, die sich in der Vergangenheit bewährt hat, trotzdem unter veränderten Umgebungsbedingungen ihre Attraktivität verlieren. Eine Polarisierung der Lebensformen einer Gesellschaft hin zu einer verstärkten Dichotomie zwischen ,Kinderhabenden' und (bewusst) ,Kinderlosen', wie sie für Deutschland in den letzten 30 bis 40 Jahren sicher konstatiert werden kann, steigert vielmehr die Dringlichkeit einer Kinderberücksichtigung im Rahmen der umlagefinanzierten RV. Verschlechtern sich des Weiteren auch noch andere Umgebungsbedingungen der Rentenversicherung (die sich z.B. in einer abnehmenden Wachstumsrate des rentenversicherungspflichtigen Einkommens manifestieren), so wird eine Ausschöpfung von möglichen Reserven der RV, eben z.B. durch die Steigerung der Effizienz, bedeutender. Außerdem steigt die Sinnhaftigkeit, aus distributiven Gesichtspunkten eventuelle systemische Benachteiligungen Kinderhabender abzubauen.

Bei gegebener Beitragssumme ermöglicht eine Beitragsstaffelung nach der Kinderzahl eine Senkung der Beiträge der Eltern, ohne dabei die Absicherung der Rentner einzuschränken. ${ }^{82}$ Unterstellt man, dass die ,Nachfrage' nach Kindern im mittleren Einkommensbereich - (leicht) positiv einkommensabhängig ist, ${ }^{83}$ so wirkt der Übergang zu einer solchen kinderabhängigen Beitragsstaffelung (c.p.) (geringfügig) geburtenfördernd. Die Bedeutung dieses Effektes hängt entscheidend von der Einkommenselastizität der (komplettierten) Kinderzahl ab. ${ }^{84}$ Ökonometrische Schätzungen dieses Wertes sind mit erheblicher Vorsicht zu interpretieren, besteht doch in den Daten regelmäßig eine positive Korrelation der Einkommenshöhe mit anderen - häufig nicht gesondert erfassten - Variablen, die ebenfalls Einfluss auf die realisierte Kinderzahl haben (dürften). So könnte die Einkommenshöhe eine Proxy-Variable für das Wissen um Verhütungstechniken sein; ferner ist das Einkommen regelmäßig positiv mit der Höhe der Opportunitätskosten einer Unterbrechung der Erwerbstätigkeit zugunsten der Kindererzie-

${ }^{82}$ Für vergleichbare Vorschläge (bei einem Beitragssatz, der die jeweiligen Beitragszahlungen bestimmt) vgl. DINKEL (1981), S. 139. Er möchte - ausgehend von einem Arbeitnehmer-Beitragsanteil von $9 \%$ - für Kinderlose einen Zuschlag von ca. 3\%, für Versicherte mit nur einem Kind einen Zuschlag von 1,5\% einführen. Zur (angeblichen) Notwendigkeit, eine eventuelle Beitragssatzstaffelung auf den Arbeitnehmeranteil zu beschränken, vgl. auch SCHMIDT und GASCH (1993), S. 272.

Vgl. BEN-PoRATH (1974), HASHIMOTO (1974), MACUNOVICH (1996), MichaEL (1974). Vgl. auch BECKER (1960), BECKER und LEWIS (1974), NERLOVE et al. (1987), S. 55 - 58.

Die ,komplettierte Kinderzahl` gibt die Zahl der Kinder nach Abschluss der Familiengründungsphase an. 
hung korreliert. Außerdem steigt die Höhe des Arbeitseinkommens tendenziell mit der Berufserfahrung - und ist somit eher höher, wenn eine vergleichsweise kurze Unterbrechung der Erwerbstätigkeit zugunsten von Kindererziehung vorliegt. Die gerade erwähnten Variablen dürften (c.p.), werden sie nicht gesondert erfasst, zu einem zu niedrigen Ausweis der (reinen) Einkommensabhängigkeit der komplettierten Kinderzahl führen.

Aber auch jenseits des reinen Einkommenseffekts einer Entlastung der Eltern bei einer rentenmäßigen Kinderberücksichtigung in Form verringerter Beitragszahlungen sind weitere Effekte möglich: Insbesondere steigt das Einkommen der Eltern während der Erziehungsphase - mit entsprechend verbesserten Möglichkeiten der intertemporalen Allokation der Finanzmittel (und auch des Lebensstandards). Damit wird die gesellschaftliche Wahrnehmung von Kindern als ,Belastung' für den (materiellen) Lebensstandard verringert. Die finanzielle Entlastung der Eltern wird ferner zu einer Zeit spürbar, in der noch die Möglichkeit einer Entscheidung zugunsten weiterer Kinder besteht.

Sollte eine Kinderberücksichtigung in Form einer Beitragsstaffelung (c.p.) zu einer Erhöhung der Kinderzahl gegenüber dem Fall einer kinderzahlunabhängigen Ausgestaltung führen, so fiele es den Kindern dann - infolge größerer Zahl der Köpfe, auf die die ,Rentnerlast' zu verteilen ist ${ }^{85}$ leichter, auch die Kinderlosen der Vorperiode in ihrem Alter mitzuversorgen. ${ }^{86}$

Eine weitere potenzielle Wirkung geringerer Beiträge ,Kinderreicher' wäre eine Erhöhung der Ersparnis auf Seiten der Eltern - und eine Steigerung der Erbschaften für die Kinder, die damit wiederum in die Lage versetzt würden, die ,Altenlast' leichter zu tragen. Auf jeden Fall trüge eine solche Regelung aber zu einer Schärfung des Bewusstseins um die Bedeutung von Kindern für die Sicherung der Tragfähigkeit einer umlagefinanzierten RV und zu einem Ausgleich externer Effekte bei - das Rentenversicherungssystem käme dem (Teil-)Ziel allokativer Effizienz näher.

${ }^{85}$ Es ist zu beachten, dass im Rahmen der gerade untersuchten Ausgestaltung der Rentenversicherung nur die Wirkung auf die Kinderzahl bedeutsam ist - eine bessere Ausbildung der Kinder etc. (,Kinderqualität') steigert infolge der pauschalierten und als intertemporal konstant unterstellten Beitragszahlung die Möglichkeit der Aufbringung von Finanzmitteln zum Unterhalt der Altengeneration nicht! Für ,realitätsnähere' Ausgestaltungsformen (wie z.B. einer einkommensabhängigen Beitragszahlung) gilt diese Aussage allerdings nicht mehr.

86 Außerdem gåbe es dann ein Bewusstsein, dass die Kinderlosen in ihrer Aktivzeit in Form erhöhter Beiträge die Eltern entlastet und somit indirekt auch die Lebensverhältnisse der Beitragszahler in ihrer Jugend verbessert haben. 


\subsection{Formale Darstellung der Kinderberücksichtigung und einige Beispielrechnungen}

Im Folgenden soll das beschriebene Verfahren einer Kinderberücksichtigung in der Beitragsphase formal dargestellt werden, um anschließend die (distributiven) Wirkungen anhand einiger (rudimentärer) Beispielrechnungen besser verdeutlichen zu können.

\subsection{1. Das Verfahren}

Der ,rentenmäßige' externe Effekt eines weiteren - Beiträge zahlenden - Kindes ${ }^{87}$ lässt sich bei einem Rentenbeitrag von $b_{t+1}$ berechnen als

$$
\underbrace{\frac{N_{t}-1}{N_{t}}}_{I} \cdot \underbrace{b_{t+1}}_{I I}
$$

Gleichung 3-5

wobei der mit $I$ unterschriebene Teil den Anteil der Beitragszahlungen des Kindes angibt, der nicht seinen Eltern zugute kommt, während der mit II unterschriebene Teil der Beitragszahlung des Kindes entspricht.

Da dieser Wert erst in der Zukunft anfällt, muss er abdiskontiert werden. Eine Thematisierung der Wahl des ,richtigen' Diskontierungsfaktors erfolgt in Abschnitt 4.3.3.2.1. Die dortigen Ausführungen lassen an dieser Stelle eine Abdiskontierung mit der ,biologischen Verzinsungsrate' der Beitragszahlungen zur GRV angemessen erscheinen, da hier eine (bezüglich der Pro-Kopf-Größen) statische Umgebung betrachtet wird. Der so bestimmte Diskontierungsfaktor $\left(D_{t, t+1}\right)$ beträgt

$\frac{N_{t}}{N_{t+1}}$

Aus den getroffenen Annahmen lässt sich der Barwert der externen Effekte eines Kindes für die RV berechnen als

$$
\frac{N_{t}-1}{N_{t}} \cdot b_{t+1} \cdot \frac{N_{t}}{N_{t+1}}=\frac{N_{t}-1}{N_{t+1}} \cdot b_{t+1} .
$$

\section{Gleichung 3-6}

87 Ist im weiteren von ,Kindern“ die Rede, so sind damit nur die künftigen Beitragszahler zur RV gemeint. 
Es stellt sich als nächstes die Frage, ob alle Kinder rentenmäßig in gleicher Höhe berücksichtigt werden sollten. Für eine derartige Ausgestaltung spricht, dass die Kinder in ihrer Aktivphase in der vorliegenden Ausgestaltung auch einen übereinstimmenden Rentenbeitrag leisten. Gegen eine von der ,Ordnungszahl' des jeweiligen Kindes unabhängige Kinderberücksichtigung lässt sich vorbringen, dass der Wert gewisser Versicherungsleistungen der Rentenversicherung und insbesondere die schon geschilderten individuellen Vorteile aus der Existenz der RV, nicht von der jeweiligen Kinderzahl abhängen. Auch Anreizgesichtspunkte mögen für eine differenzierende Kinderberücksichtigung sprechen. Für das weitere wird trotzdem von einer einheitlichen Kinderberücksichtigung ausgegangen, zumal sich eine Gewichtung der Kinder nach Ordnungszahl in der folgenden Abschätzung grundsätzlich implementieren lässt, ohne am Grundmuster der Ausgestaltung wesentliches zu ändern. Sie führt aber zu deutlich aufwendigerer Notation, ohne fundamental neue Erkenntnisse zu vermitteln. Ferner wird - insbesondere aus Gründen der darstellerischen Klarheit, und trotz der schon genannten Gründe für einen nur partiellen Ausgleich - von einem vollständigen Ausgleich der ,externen Effekte“ des Kinderhabens ausgegangen.

Wird die Beitragssumme durch die Kinderberücksichtigung nicht verändert, ergibt sich bei einer einheitlichen rentenmäßigen Berücksichtigung aller Kinder und einer vollständigen Internalisierung der (rentenmäßigen) externen Effekte die Bedingung

$$
N_{t} \cdot b_{t} \stackrel{!}{=} \sum_{n}\left\{N_{t, n} \cdot\left(b^{0}-n \cdot b_{t+1} \cdot \frac{N_{t}-1}{N_{t+1}}\right)\right\}
$$

(mit $b^{0}$ : Rentenversicherungsbeitrag eines Beitragszahlers mit 0 Kindern; $N_{t, n}$ : Zahl der Beitragszahlers in $t$ mit $n\left(n \in N^{0}\right)$ Kindern).

Diese Gleichung lässt sich umformen zu

$$
N_{t} \cdot b_{t} \stackrel{!}{=} b^{0} \cdot \sum_{n} N_{t, n}-b_{t+1} \cdot \frac{N_{t}-1}{N_{t+1}} \cdot \sum_{n}\left\{N_{t, n} \cdot n\right\}
$$

und wegen $\sum_{n} N_{t, n}=N_{t}$ und $\sum_{n}\left\{N_{t, n} \cdot n\right\}=N_{t+1}$ zu

$$
N_{t} \cdot b_{t} \stackrel{!}{=} b^{0} \cdot N_{t}-b_{t+1} \cdot\left(N_{t}-1\right) .
$$


Hieraus lässt sich $b^{0}$ für den Fall eines intertemporal konstanten durchschnittlichen Beitrags $\left(b_{t}=b_{t+1} \equiv b\right)$ berechnen als

$$
b^{0}=2 \cdot b-\frac{1}{N_{t}} \cdot b
$$

\author{
Gleichung 3-10
}

Für $b^{n}$ (Rentenversicherungsbeitrag bei $n$ Kindern) folgt hieraus:

$$
b^{n}=2 \cdot b-\frac{1}{N_{t}} \cdot b-n \cdot \frac{N_{t}-1}{N_{t+1}} \cdot b \cdot{ }^{88}
$$

\title{
Gleichung 3-11
}

Der Gleichung lässt sich für eine Gesellschaft mit konstantem Umfang der Aktivgeneration $\left(N_{t}=N_{t+1}\right)$ entnehmen, dass jedes Kind den Rentenbeitrag um ungefähr die Hälfte des Beitrags Kinderloser verringert. ${ }^{89}$

\subsection{2. Exemplarische Beispielrechnungen}

Im Folgenden werden jeweils für eine zunehmende und eine abnehmende Generationenstärke die Renten(beitrags)zahlungen ohne und mit erfolgender Kinderberücksichtigung beispielhaft berechnet.

Fall: Zunehmender Generationenumfang, keine Kinderberücksichtigung

Zum Zeitpunkt $t$ gibt es 1.000 Beitragszahler; die intertemporal konstante durchschnittliche Beitragszahlung zur RV beträgt 1.000 Geldeinheiten (GE). Die 1.000 Beitragszahler erziehen 1.100 Kinder; eine Kinderberücksichtigung in der RV findet nicht statt. In $t+1$ bezieht jeder Rentner eine Rente von $1.100 \mathrm{GE}$.

Fall: Zunehmender Generationenumfang, Kinderberücksichtigung

Zum Zeitpunkt $t$ gibt es 1.000 Beitragszahler; die intertemporal konstante durchschnittliche Beitragszahlung zur RV beträgt $1.000 \mathrm{GE}$. Die 1.000 Beitragszahler

${ }^{88}$ Es gilt mithin $\left(N_{t}, N_{t+1} \gg 0\right): b^{n} \approx 2 \cdot b-n \cdot \frac{N_{t}}{N_{t+1}} \cdot b$.

${ }^{89}$ Aus $N_{t}=N_{t+1}$ und $N_{t}>>$ folgt: $b^{n} \approx 2 \cdot b-n \cdot b$. 
erziehen 1.100 Kinder; es gibt eine Kinderberücksichtigung in der RV. Der ,externe rentenmäßige Kinderwert' lässt sich berechnen als (ca.) 908,18 GE. Die Beitragszahlung eines Versicherten mit 0 Kindern beträgt $1.999 \mathrm{GE}$, die eines Versicherten mit 1 Kind (ca.) 1.090,82 GE, die eines Versicherten mit 2 Kindern (ca.) 182,64 GE, und für einen Versicherten mit 3 Kindern liegt der Wert bei ca. 725,54 GE, d.h. der Versicherte erhält schon in der ,Beitragsphase' Zahlungen der Rentenversicherung! In $t+1$ bezieht jeder Rentner eine Rente von 1.100 GE.

Fall: Abnehmender Generationenumfang, keine Kinderberücksichtigung

Zum Zeitpunkt $t$ gibt es 1.000 Beitragszahler; die intertemporal konstante durchschnittliche Beitragszahlung zur RV beträgt $1.000 \mathrm{GE}$. Die 1.000 Beitragszahler erziehen 900 Kinder; eine Kinderberücksichtigung in der RV findet nicht statt. In $t+1$ bezieht jeder Rentner eine Rente von $900 \mathrm{GE}$.

Fall: Abnehmender Generationenumfang, Kinderberücksichtigung

Zum Zeitpunkt $t$ gibt es 1.000 Beitragszahler; die intertemporal konstante durchschnittliche Beitragszahlung zur RV beträgt $1.000 \mathrm{GE}$. Die 1.000 Beitragszahler erziehen 900 Kinder; es gibt eine Kinderberücksichtigung in der RV. Der ,externe rentenmäßige Kinderwert' lässt sich berechnen als $1.110 \mathrm{GE}$. Die Beitragszahlung eines Versicherten mit 0 Kindern beträgt $1.999 \mathrm{GE}$, die eines Versicherten mit 1 Kind (ca.) $889 \mathrm{GE}$, die eines Versicherten mit 2 Kindern - $221 \mathrm{GE}$ etc. In $t+1$ bezieht jeder Rentner eine Rente von $900 \mathrm{GE}$.

\subsection{Rentenstaffelung nach Kinderzahl}

Eine ausschließliche (proportionale) Staffelung der Rentenhöhe nach der Kinderzahl ist für ein Zwangsrentensystem unter der Bedingung einer vollständigen Internalisierung der externen rentenmäßigen Effekte des Kinderhabens kein gangbarer Weg: Da hierbei die Pro-Kopf-Beitragszahlungen der Nachfolgegeneration eines Rentners direkt proportional zur Zahl der Kinder steigen, bedeutet dies, dass eine direkte Umverteilung von den Kindern an ihre Eltern erfolgt. Eine Absicherung Kinderloser ist hierbei nicht möglich; außerdem ist zu fragen, warum bei einer derartigen Ausgestaltung nicht gleich auf die rein ,innerfamiliäre' Altersabsicherung zurückgegriffen wird, d.h. der Umweg des Transfers vom Kind an seine Eltern über den Umweg Rentenversicherung erfolgt. Bestenfalls ließe sich hier in Abwesenheit jedweder ,Versicherung' - mit einer Einsparung von (psychi- 
schen?) Transaktionskosten argumentieren. Eine derartige Argumentation erscheint allerdings zweifelhaft. ${ }^{90}$

\subsubsection{Version 2 eines Umlageverfahrens unter statischen Bedingungen}

Im Folgenden soll zuerst der Übergang von Version 1 eines Umlageverfahrens zu Version 2 aufgezeigt werden, um dann auf die Kinderberücksichtigung in Version 2 einzugehen. Die geringe praktische Bedeutung dieses Ansatzes - verbunden mit dem eingeschränkten ,Neuigkeitsgehalt" - erlaubt hierbei eine eher summarische Thematisierung.

\subsubsection{Beziehungen zwischen Version 1 und Version 2}

Der im letzten Abschnitt dargestellte Ansatz verdeutlicht die Möglichkeit einer Kinderberücksichtigung im Rahmen der als Version 1 charakterisierten Grundform des Umlageverfahrens (intertemporal konstante durchschnittliche Beitragszahlungen bei variierenden Rentenzahlungen). Das obendrein unterstellte intertemporal konstante (reale) Pro-Kopf-Einkommen der Aktiven ermöglicht eine Ausklammerung der Frage einer laufenden Anpassung der Renten- und Beitragshöhe ${ }^{91}$ zur Sicherung eines ,angemessenen' Verhältnisses einer ,durchschnittlichen Rente' zu einem ,durchschnittlichen Einkommen' eines Mitglieds der Aktivgeneration. Bleibt die Änderungsrate des Generationenumfangs intertemporal konstant, so ändert sich an den relativen Einkommenspositionen repräsentativer Mitglieder der Rentner- und der Aktivgeneration nichts.

Infolge der unterstellten Konstanz aller rentenrelevanten Parameter kann der gerade vorgestellte Fixbeitragsansatz ohne größere Probleme in einen Fixrentenansatz (Version 2) transformiert werden. Kennzeichen des letzteren ist eine intertemporal konstante Höhe der Rente eines jeweiligen Rentners. ${ }^{92}$ Somit ist mit dem Umfang der Rentnergeneration auch die aufzubringende Rentensumme gegeben.

90 Für einen Ansatz, der eine partielle - wenn auch nicht einheitliche - Berucksichtigung von Kindern bei der Rentenzahlung inkludiert, vgl. Kap. 4. Dort wird insbesondere auch eine Rentenzahlung auch an Kinderlose begrundet und untersucht.

Unter Berücksichtigung der Budgetrestriktion.

92 Version 2 entspricht der Logik einer umlagefinanzierten Grundsicherung jedes Rentners durch eine ,Mindestrente', die unabhängig von den sonstigen Einkommens- und Vermögensverhältnissen (und ohne Ruckgriff auf Einkommen und / oder Vermögen von Verwandten des Rentenbeziehers) gezahlt wird. 
Bei übereinstimmenden (rentenversicherungsrelevanten) Charakteristika der Mitglieder der Aktivgeneration (und gegebenem Umfang der Aktivgeneration) folgt daraus automatisch der Rentenbeitrag eines jeden Aktiven.

Bei interindividuellen Unterschieden zwischen den Mitgliedern der Aktivgeneration gibt die vorangegangene Leitlinie den durchschnittlichen Rentenbeitrag eines Mitglieds der Aktivgeneration an.

\subsubsection{Kinderberücksichtigung in Version 2}

Im Folgenden soll - kurz - auf die Möglichkeit einer Kinderberücksichtigung in einer nach Version 2 ausgestalteten Rentenversicherung eingegangen werden. ${ }^{93}$ Die Vorgehensweise ist analog zu der in Abschnitt 3.2.1.3. Dort wurde eine nach der Zahl der Kinder gestaffelte Beitragszahlung in der Aktivphase bei gegebener durchschnittlicher Beitragszahlung und einer übereinstimmenden Rentenhöhe im Alter dargestellt. In der grundsätzlichen Ausgestaltung ist damit also bereits eine nicht unerhebliche Annäherung an eine sich an Version 2 anlehnende Organisation der Rentenversicherung erfolgt. Es darf aber nicht übersehen werden, dass bei der modifizierten Ausgestaltung nach Version 1 nur intragenerational eine einheitliche Rente gesichert ist; Änderungen im Reproduktionsverhalten finden einen entsprechenden Niederschlag in der Rentenhöhe. Bei einer Ausgestaltung nach Version 2 hingegen stimmt die Rentenhöhe sowohl intra- als auch intergenerational überein: Änderungen der Reproduktionsrate haben damit Einfluss auf die Belastung der nachfolgenden Generation. Auf eine Wiedergabe einer formalen Darstellung soll im Haupttext verzichtet werden - die wesentliche ,formelmäßige' Änderung ist, dass $b_{t+1}(=b)$ durch $r \cdot \frac{N_{t}}{N_{t+1}}$ ersetzt (und somit endogenisiert) wird, wobei jetzt die Rentenhöhe $r$ exogen gegeben ist. Für Einzelheiten sei auf die Darstellung in Anhang 1 (Abschnitt 7.1) sowie auf den Nachweis der Übereinstimmung der Kinderberücksichtigungen der beiden Ansätze (unter einfachen Annahmen) in Anhang 2 (Abschnitt 7.2) verwiesen.

\subsubsection{Wirkungen von Änderungen in der Wachstumsrate der Stärke der Aktivgeneration in den Versionen 1 und 2}

Die vorausgesetzte Übereinstimmung der Kinderberücksichtigung in den Versionen 1 und 2 ist im Falle sich ändernder generativer Verhaltensweisen nicht gegeben. Hierauf sei im nachfolgenden Abschnitt näher eingegangen. Zur Darstellung

93 Die Begründung der Angemessenheit einer Kinderberücksichtigung in Version 2 entspricht der in Version 1, so dass hier auf eine Darstellung verzichtet werden kann. 
der Unterschiede werden zuerst die Wirkungen einer Ausgestaltung nach Version 1 und Version 2 kurz zusammengefasst, um dann zu einem Vergleich der beiden Ausgestaltungen überzugehen. Dabei wird weiterhin ein intertemporal konstantes Pro-Kopf-Einkommen der Aktiven unterstellt.

\subsection{Darstellung und Vergleich der Belastungswirkungen}

Änderungen in der Wachstumsrate $\left(g_{t}\right)$ der ,aktiven' Bevölkerung führen bei intertemporal fixierter individueller Beitragshöhe $\left(\forall t: b_{t} \equiv b\right) \mathrm{zu}$ einer proportionalen Änderung der Pro-Kopf-Rentenzahlung an die Rentnergeneration (die Pro-Kopf-Rente beträgt in der Periode $t+1: r_{t+1}=\frac{N_{t+1}}{N_{t}} \cdot b=\left(1+g_{t}\right) \cdot b$; hieraus folgt: $\frac{d r_{t+1}}{d g_{t}}=b$ ). Durch eine Zunahme der Wachstumsrate der Aktivbevölkerung steigt bei konstantem Rentenbeitrag der Aktiven das Renteneinkommen der Rentner - und zwar sowohl absolut, als auch relativ zum Einkommen der Aktiven. Wird hingegen eine konstante Rentenhöhe eines repräsentativen Rentners vorgegeben $\left(\forall t: r_{t} \equiv r\right)$, so führen Änderungen in der Wachstumsrate der Stärke der Aktivgeneration zu einer gegengerichteten Änderung der Beitragsverpflichtung für ein repräsentatives Mitglied der Aktivgeneration: ${ }^{94}$ Bei konstanter Rentenhöhe ergibt sich $\quad r=\frac{N_{t+1}}{N_{t}} \cdot b_{t+1} \Leftrightarrow b_{t+1}=\frac{N_{t}}{N_{t+1}} \cdot r=\frac{1}{1+g_{t}} \cdot r ; \quad$ hieraus folgt: $\frac{d b_{t+1}}{d g_{t}}=-\frac{r}{\left(1+g_{t}\right)^{2}}<0$.

Bleibt die Wachstumsrate des Umfangs der Aktivbevölkerung im Zeitablauf unverändert, so verschiebt sich auch das zahlenmäßige Verhältnis von Rentenbeziehern zu Rentenbeitragszahlern im Zeitablauf nicht. Bei Konstanz des ProKopf-Einkommens eines durchschnittlichen Mitglieds der Aktivgeneration kommt es (siehe auch Anhang 2) mithin weder bei Version 1 noch bei Version 2 zu einer Änderung des Verhältnisses von durchschnittlicher Rentenhöhe zum durchschnittlichen Aktiveinkommen (nach Beitragszahlung zur RV). Die sich aus den intertemporal konstanten Änderungsraten des Generationenumfangs ergebenden Rentenwirkungen brauchen nur ,einmalig' bei der Festlegung des Rentenbeitrags bzw. der Rentenhöhe berücksichtigt zu werden - es kommt dann im Zeitablauf zu keinen Änderungen in der Belastung der Mitglieder der jeweiligen Generation.

94 Infolge konstanter (durchschnittlicher) Pro-Kopf-Einkommen verschlechtern höhere Beitragsverpflichtungen der Aktiven auch deren relative ,Netto'-Einkommensposition. 
Nimmt die Wachstumsrate des Umfangs der Aktivgeneration hingegen ab, ${ }^{95}$ so kommt es in Version $1 \mathrm{zu}$ einer sinkenden absoluten (und relativen) Rentenhöhe, während in Version 2 die individuelle Beitragszahlung eines Mitglieds der Aktivgeneration steigt; wegen der höheren Rentenbeitragszahlung der Aktiven bei unverändertem (Brutto-)Einkommen und konstanter Rentenhöhe verbessert sich damit die relative Einkommensposition eines repräsentativen Rentners im Vergleich zu einem repräsentativen Aktiven. Die beiden Verfahren unterscheiden sich also in der intergenerationalen Verteilung der den Mitgliedern des Rentenversicherungssystems aus einem rückläufigen Bevölkerungswachstum resultierenden Finanzlast: Während Version 1 die Rentnergeneration belastet, wird bei Version 2 die Aktivgeneration, in die Pflicht genommen'.

\subsubsection{2. $\quad$ Abwägung der Vorteilhaftigkeit einer Ausgestaltung nach Version 1 und Version 2 im Falle einer Änderung der Wachstumsrate}

Die unterschiedlichen intergenerativen Belastungseffekte einer Beitragsfixierung (Version 1) und einer Rentenfestlegung (Version 2) bei sich ändernden Wachstums- resp. Schrumpfungsraten lassen fragen, welche der beiden Lastenaufteilungen angemessen ist. Für die Beantwortung dieser Frage ist ein Maßstab der ,richtigen' intergenerationalen Lastverteilung zu suchen. Aus allokativer Perspektive ist zu fordern, dass die ökonomischen Konsequenzen einer Handlung (Unterlassung) möglichst denjenigen zugerechnet werden, die die Entscheidung über die Handlung getroffen haben. Nur bei Internalisierung der externen Effekte besteht die Möglichkeit einer Entscheidung unter Berücksichtigung ihrer gesamten ökonomischen Folgen. Außerdem kann es als ein Gebot distributiver Gerechtigkeit angesehen werden, dass diejenigen, die Kosten der Kindererziehung eingespart haben, im Alter dann die aus diesen niedrigeren ,Investitionen' resultierenden (geringeren) Erträge ,zu spüren' bekommen.

Die jeweilige Rentnergeneration in toto hat sich im Fall einer abnehmenden Wachstumsrate während ihrer Aktivzeit zugunsten einer geringeren durchschnittlichen Kinderzahl entschieden. Für die Gruppe als Ganze sprechen mithin die Forderungen allokativer Effizienz wie distributiver Gerechtigkeit für eine Rentenberechnung nach Version 1, also für eine Tragung der Kosten eines verringerten Wachstums der Aktivgeneration durch die Elterngeneration in deren Rentenphase. Allerdings ist zu beachten, dass die Änderung der Wachstumsrate des Generationenumfangs Resultat einer Vielzahl individueller Entscheidungen (potenzieller)

95 Um das Nachvollziehen der Belastungsunterschiede einer Änderung in der Wachstumsrate der Aktivbevőlkerung zu erleichtern, wird im Folgenden ausschließlich auf den Fall einer abnehmenden Wachstumsrate eingegangen. (NB: An dieser Stelle wird auf die Änderung der Wachstumsrate abgestellt, nicht auf das Vorzeichen der Wachstumsrate!) 
Eltern über ihre jeweilige Kinderzahl ist. Da die Eltern ihre generative Entscheidung unter Berücksichtigung ihrer individuellen Kosten und Nutzen des Kinderhabens treffen, sichert die Zurechnung der rentenmäßigen Lasten einer verringerten durchschnittlichen Kinderzahl auf die gesamte Elterngeneration (ohne Kinderstaffelung der Beiträge) infolge der Möglichkeit eines free-riding nicht eine individuelle Entscheidung zugunsten einer allokativ effizienten durchschnittlichen Kinderzahl (Rationalitätenfalle).

Eine Begründung für die Ausgestaltung des Umlageverfahrens nach Version 1 ist somit zuerst in unterschiedlichen distributiven Wirkungen der beiden Ansätze zu finden. In beiden hier vorgestellten Verfahren sind die Kosten der Kindererziehung nicht für die Rentenhöhe entscheidend. Sie sind von den Eltern (bzw. den Mitgliedern der Elterngeneration) zu tragen. Ein Sinken der durchschnittlichen Kinderzahl bedeutet nun (c.p.) in einer Gesellschaft, in der die Kindererziehung Geld kostet (in der es also keine Selbstfinanzierung der Kinder z.B. in Form von Kinderarbeit gibt), dass die Elterngeneration (c.p.) niedrigere Unterhaltskosten hat: Die eingesparten Mittel stehen für andere Ausgabemöglichkeiten zur Disposition - insbesondere auch zur (individuellen oder institutionalisierten) Bildung von Rücklagen für das Alter. ${ }^{96}$ Bei einer Ausgestaltung nach Version 1 werden die Lasten einer verringerten durchschnittlichen Kinderzahl - in Form einer verringerten Rentenhöhe - gerade der Generation zugerechnet, die auch die Kostenersparnis aus dem veränderten generativen Verhalten in ihrer Aktivzeit realisieren konnte. Die am veränderten Generationenumfang , unschuldige' Kindergeneration wird hingegen weder be- noch entlastet. In einer der Version 2 folgenden Ausgestaltung hingegen kann eine Generation aus der Verringerung der durchschnittlichen Kinderzahl einen eindeutigen (finanziellen) Vorteil ziehen: Während die durchschnittlichen Kinderkosten pro Mitglied der Aktivgeneration sinken, ändert sich die Rentenhöhe nicht. Dafür muss hingegen die nachfolgende Generation für die Entscheidungen ihrer Elterngeneration in Form höherer Beiträge ,büßen' ohne dass sie selbst systemisch Kostenvorteile durch diese Entscheidung hatte. ${ }^{97}$

96 Es gibt unterschiedliche Möglichkeiten, um der nachfolgenden Generation die Tragung der ,Altenlast' zu erleichtern: Einerseits Investitionen in das quantitative und das qualitative Humankapital, zum anderen eine gute Ausstattung der Aktiven mit Sachkapital. Insoweit ist ein (partieller) Ausgleich geringerer Humankapitalinvestitionen durch höhere Sachkapitalinvestitionen möglich. Ferner ist zu beachten, dass - jenseits des hier unterstellten ubereinstimmenden Pro-Kopf-Einkommens der Mitglieder der Aktivgeneration - ein erhöhtes qualitatives Humankapital pro Kopf der nachfolgenden Generation einen Grund für erhöhte Pro-Kopf-Beitragszahlungen in der Aktivzeit (als ,Rückzahlung' der erhöhten Pro-Kopf-Investition der Eltern) bieten kann.

Es ist darauf hinzuweisen, dass individuell eine Entlastung der Kinder durch eine Erhöhung der Erbschaften möglich ist. Ein solcher intrafamiliärer Lastenausgleich ist insbesondere im Falle altruistischer Individuen wahrscheinlich. Allerdings ist zu beachten, dass die konkrete Ausge- 
Distributive Überlegungen sprechen folglich für eine Ausgestaltung der Rentenversicherung nach Version 1 . Sie werden noch dadurch verstärkt, dass die bei einer Ausgestaltung nach Version 2 durch erhöhte Rentenversicherungsbeiträge belasteten Mitglieder der Nachfolgegeneration ihrerseits auf die gesteigerte Belastung durch eine Anpassung ihres generativen Verhaltens reagieren: Eine mögliche - individuell naheliegende - Reaktion auf die erhöhte finanzielle Belastung liegt in einer Verringerung der Fertilität - und damit der Kinderkosten -, um so Teile des erhöhten Rentenversicherungsbeitrags ,gegenzufinanzieren'. ${ }^{98}$ Dem System fehlt eine inhärente Stabilisation!

Auch im Falle steigender durchschnittlicher Kinderzahl verdient eine Ausgestaltung der Rentenversicherung nach Version 1 distributiv (unter im wesentlichen statischen Bedingungen) den Vorzug vor einer nach Version 2: Bei intertemporal konstanten Rentenbeitragszahlungen eines repräsentativen Mitglieds einer Generation führt eine Erhöhung der durchschnittlichen Kinderzahl (c.p.) zu einer Steigerung der Rente. Die Generation, die von dieser Rentenanpassung ,profitiert' ist aber genau die Generation, die zuvor die erhöhten Kosten der Kindererziehung getragen hat.

\subsection{Der Aussagegehalt der Versionen 1 und 2 kritisch hinterfragt}

Die beiden bisher untersuchten Versionen einer im Umlageverfahren organisierten (Pflicht-)Rentenversicherung stellen für ,stationäre' Gesellschaften einen guten Ansatz zur Verdeutlichung der Bedeutung von Kindern für ein umlagefinanziertes Alterssicherungssystem dar. Insbesondere lässt sich mit seiner Hilfe die Bedeutung von Beitragszahlungen einerseits und des Kinderhabens andererseits verdeutlichen, und ebenso die Externalisierung wesentlicher Teile der (positiven) Wirkungen des Kinderhabens für die Rendite des UV. Dies wiederum gibt den Anstoß - zunächst allerdings nur innerhalb der restriktiven Annahmen - zu einer Kinderberücksichtigung, die den beitragsähnlichen Charakter des Kinderhabens in Form einer Beitragsermäßigung verdeutlicht. Der Wert der Kinderberücksichtigung (pro Kind) ist insoweit systemisch, als er dem Wert eines Kindes für die RV entspricht - es kommt mithin zu einer Internalisierung der externen Effekte des

staltung des Rentenversicherungssystems, und hier insbesondere die jeweilige Berücksichtigung von Kindern, entscheidende Bedeutung furr die jeweilige Möglichkeit eines intrafamiliären Lastenausgleichs hat. Insbesondere der Verzicht auf jede Kinderberücksichtigung verringert den finanziellen Spielraum der Eltern zugunsten einer Einkommensübertragung an ihre Kinder. menstransfers an die Alten vgl. WEIL (1999). Vgl. auch ARTHUR und MCNICOLL (1977). 
Kinderhabens für die RV; aus allokativer Sicht werden in der RV bezüglich der Höhe der Kinderzahl dann keine Fehlanreize gesetzt.

Der Analyserahmen ist aber nicht in der Lage, unsichere Einkommen bzw. Beitragszahlungen der Kinder zu berücksichtigen: Eine einheitliche Kinderberücksichtigung setzt immer dann, wenn die spätere Beitragsposition (bzw. das Einkommen) eines Kindes - auch - vom Verhalten der Eltern abhängt (Ausgaben für das Kind, Zuwendung etc.), allokativ falsche Anreize. Denn während in diesem Fall sowohl die Zahl der Kinder als auch ihre Beitragsposition für die ,Rentabilität' der RV von Bedeutung sind, findet ausschließlich eine Berücksichtigung der Kinderzahl statt; es kommt mithin zum allokativen Fehlanreiz, zu sehr auf ,Kinderquantität' und zu wenig auf ,Kinderqualität' zu achten (,Masse statt Klasse').

\subsection{Vergleich der beiden Ansätze zur Kinderberücksichtigung}

Jenseits der Frage, ob eine (vollständige) Internalisierung der externen Effekte des Kinderhabens im Rahmen einer Rentenversicherung möglich und geboten ist, und unabhängig von der konkreten Ausgestaltung einer Kinderberücksichtigung, bleibt noch zu diskutieren, inwieweit eine im Vergleich mit einem repräsentativen Mitglied der jeweiligen Generation unterdurchschnittliche Fertilität ,beitragserhöhend' oder ,rentensenkend' wirken soll. ${ }^{99}$

Die grundsätzlichen allokativen Vorteile einer Kinderberücksichtigung schon in der Beitragsphase sind bereits thematisiert worden (vgl. 3.2.1.3.1.1). An dieser Stelle sei aber noch auf zwei weitere Punkte hingewiesen: Steht den Eltern in der Erziehungsphase mehr Geld zur Verfügung, so können sie damit ,Bildungsinvestitionen' zugunsten ihrer Kinder tätigen. ${ }^{100}$ Gerade bei der Finanzierung solcher Investitionen ist mit einem Marktversagen zu rechnen, da es keine ,natürliche Sicherheit' für eventuelle Kredite zur Ausbildungsfinanzierung gibt, so dass bei kreditbeschränkten Familien ein Auseinanderfallen der Verzinsung von Bildungsinvestitionen und des Sollzinssatzes à priori nicht auszuschließen ist. ${ }^{101}$ Aus ge-

99 Aus Gründen der argumentativen Klarheit werden nur die beiden ,reinen' Strategien betrachtet.

$100 \mathrm{Vgl}$. WERDING (1998), S. $162 \mathrm{f}$.

${ }^{101}$ Eine Tendenz zur Unterlassung ,rentabler' Investitionen in das (qualitative) Humankapital ihrer Kinder konstatieren AGEE und CROCKER (1996) fur ärmere Familien - die ,Rendite' liegt hier etwa beim doppelten des Marktzinssatzes für langfristige Anlagen, während die Zinssätze bei ,vermögenderen Familien' in etwa übereinstimmen. (Die Untersuchung bezieht sich allerdings auf die USA mit den dortigen Ausgestaltungen der Finanzierung schulischer und universitärer Bildung.) 
samtgesellschaftlicher Sicht ist dem allerdings für die gegenwärtige deutsche Ausgestaltung der Bildungsfinanzierung entgegenzuhalten, dass die Gemeinschaft der Steuerzahler schon erhebliche Teile der ,Ausbildungskosten' übernimmt, so dass von daher die individuellen Kosten der Inanspruchnahme von Bildungseinrichtungen unter den ,wahren' Kosten liegen; somit ist ein ,Überkonsum' von Bildung nicht auszuschließen. Allerdings wäre auch in diesem Fall eine an der Liquidität orientierte Beschränkung des Bildungserwerbs allokativ wohl kaum eine erstbeste Maßnahme zur Vermeidung überhöhter Nachfrage nach Bildung, und eine Relaxation eventueller Ausbildungshemmnisse von daher vorteilhaft.

Der zweite hier zu erwähnende Vorteil der Kinderberücksichtigung schon in der Beitragsphase besteht in der ,Sicherheit' der erhaltenen Einkommenssteigerung. Während eine höhere Rente infolge der Kindererziehung nur denjenigen zugute kommt, die das Rentenalter erreichen, profitieren von einer Berücksichtigung schon in der Aktivphase auch Eltern, die infolge frühen Todes niemals eine Rente beziehen werden. Bei - wohl zu unterstellender - Risikoaversion ist auch von daher auf Seiten der Eltern eine Präferenz für eine Kinderberücksichtigung in der Aktivphase geben. ${ }^{102}$ Außerdem ist eine Entlastung schon in der Beitragsphase intuitiv spürbarer als eine Kinderberücksichtigung erst in der Rentenphase.

Es ist abschließend kurz darauf hinzuweisen, dass die gerade thematisierten Vorteile einer Kinderberücksichtigung schon in der Aktivphase primär Folge von Kapitalmarktunvollkommenheiten, unsicherer Lebenserwartung etc. sind. Sie folgern aus der faktischen Unmöglichkeit einer (fast) vollständigen Verpfändung zukünftigen (erwarteten) Einkommens (Vorziehung von Zukunftskonsum in die Gegenwart), einer positiven Differenz zwischen Passiv- und Aktivzinsen sowie interindividuell unterschiedlicher Lebensdauer. Insoweit sind sie für die ursprünglichen Modellbedingungen unbedeutend. Unter realistischen Annahmen ist ihre Bedeutung hingegen erheblich.

${ }^{102}$ Diese Feststellung gilt unabhängig davon, ob im Falle einer Kinderberücksichtigung erst in der Rentenphase durch frühzeitigen Tod von Rentenberechtigten eingesparte Mittel der Kinderberulcksichtigung an alle Versicherten, oder aber nur an die Bezieher von Kinderrenten ausgeschüttet werden. Im Extremfall könnten allerdings bezüglich ihrer ,Überlebenswahrscheinlichkeit' optimistische Eltern im Einzelfall trotz Risikoaversion fur eine Kinderberucksichtigung erst in der Rentenphase eintreten, erwarten sie dann doch, von der Konzentration der Finanzmittel auf eine recht geringe Zahl von Versicherten zu profitieren. In Anbetracht der schon erwähnten Nachteile einer Kinderberücksichtigung erst im Alter erscheint die Bedeutung dieses Falls jedoch vernachlässigbar gering. 


\subsection{Versionen mit relativen Absicherungs- oder Belastungsvorgaben}

Im Folgenden sollen Version 3 und Version 4 eines Umlageverfahrens genauer untersucht werden. Während die Beitragszahlung in der vorgestellten Version 1 einer umlagefinanzierten RV fix ist, wird sie in beiden Ansätzen als variabel modelliert. Diese Variabilität erschwert die Kinderberücksichtigung - wie sich im weiteren zeigen wird - erheblich. Zur Untersuchung wird zuerst die Grundidee eines Beitragssatzverfahrens einer umlagefinanzierten RV (Version 3) dargestellt. Dem schließt sich eine Analyse der ,externen rentenmäßigen Effekte' des Kinderhabens an. Es folgt ein Vergleich mit der Bedeutung des Kinderhabens im Fixbeitragsansatz (Version 1), der überleitet zur Darstellung der Probleme einer adäquaten Kinderberücksichtigung im Beitragssatzverfahren. Anschließend erfolgt eine entsprechende Untersuchung von Version 4. Am Anfang des Abschnitts steht jedoch eine Darstellung der Gründe, die ein Verlassen des in Version 1 und Version 2 genutzten Annahmerahmens nahelegen.

\subsubsection{Gründe für eine Analyse der Versionen 3 und 4}

\subsubsection{Teilhabe der Rentner an Produktivitätsfortschritten}

Wie bereits bei der Vorstellung der vier verschiedenen idealtypischen Ausgestaltungsformen einer umlagefinanzierten Rentenversicherung erwähnt, haben Ausgestaltungen nach Version 1 bzw. 2 für Gesellschaften mit sich im Zeitablauf ändernden durchschnittlichen Realeinkommen der Mitglieder der Aktivgeneration (Produktivitätssteigerung bzw. -senkung) den bedeutsamen Nachteil, dass es ohne Änderungen in der Wachstumsrate der Generationenstärke - zu einer Entkopplung der Höhe der Aktiveinkommen und der Renteneinkommen kommt.

$\mathrm{Da}$ an dieser Stelle ausschließlich die sich aus Änderungen der Produktivität ergebenden Rentenänderungen betrachtet werden, also die aus Änderungen des generativen und erzieherischen Verhaltens folgenden Konsequenzen ausgeblendet werden, ${ }^{103}$ erscheint es angemessen, im weiteren primär auf den - bedeutender erscheinenden - Fall realisierter Produktivitätsfortschritte der Aktivgeneration

${ }^{103}$ Diese sind in einer Ausgestaltung der Rentenversicherung nach Version 1 als Änderungen des ,Zinseinkommens' auf das in der Aktivzeit investierte quantitative ,Humankapital' der Kinder zu deuten. Hat eine Rentnergeneration in ihrer Aktivzeit besonders viel in das Humankapital der nachfolgenden Generation investiert, so ist eine sich daraus ergebenden Verbesserung ihrer Einkommensposition im Alter als Rückzahlung eines intertemporalen Transfers zu verstehen.. 
einzugehen. Bei andauerndem Produktivitätsfortschritt sinkt die relative Einkommensposition eines repräsentativen Rentners (sowohl bei einer Ausgestaltung nach Version 1 als auch bei einer nach Version 2) - verglichen mit einem repräsentativen Aktiven - kontinuierlich. Dient die Rente nicht nur der Vermeidung von absoluter Armut eines Versicherten in seiner Altersphase - bei durchschnittlichen existenznotwendigen Aufwendungen -, sondern hat sie auch das Ziel einer Absicherung des (relativen) Lebensstandards der Alten, also auch einer Partizipation an möglichen Steigerungen des Wohlstands, so erweisen sich die beiden bisher untersuchten Versionen als ungeeignet. Grundsätzlich wäre ihre Modifikation mit dem Ziel einer Lebensstandardabsicherung und einer Verhinderung der Überforderung der Aktivgeneration möglich: Hierfür müssen der Fixbeitrag resp. die Fixrente im Zeitablauf nur, angemessen' variiert werden - z.B. entsprechend der Änderungsrate des Nettolohnes der Aktiven. In einer Gesellschaft identischer Individuen mit intertemporalem Produktivitätsfortschritt wäre eine Lebensstandardsicherung für das Alter also auch in einem nur leicht modifizierten Rentenmodell nach Version 1 möglich (wobei sich auch hier das Problem ergibt, die aus verringerter durchschnittlicher Kinderzahl resultierende Verschlechterung der relativen Einkommensposition im Alter nicht durch Erhöhung der Rentenbeiträge zu Lasten der Aktivgeneration ,aufzufangen'). Insoweit erscheint der Übergang zu einer Ausgestaltung der GRV nach den Versionen 3 bzw. 4 nicht unbedingt systematisch nötig. Besonderheiten bei einer Ausgestaltung nach Version 3 oder 4 ergeben sich hingegen, wird von der Annahme intragenerational übereinstimmender Arbeitseinkommen der Aktiven abgegangen, und wird gleichzeitig an die Rentenversicherung die Anforderung einer Absicherung des (relativen) Lebensstandards für die Ruhestandsphase gestellt. Spätestens an dieser Stelle bietet sich dann eine (teilweise) Kopplung der Höhe der Beitragszahlungen in der Aktivphase an das in dieser Phase erzielte Einkommen an. ${ }^{104}$

\subsubsection{Induzieren in einem Umlageverfahren Beitragszahlungen Rentenansprüche?}

Bevor weiterhin eine Kinderberücksichtigung im Rahmen eines umlagefinanzierten Rentenversicherungsystems thematisiert wird, ist allerdings zu klären, ob Beitragszahlungen in dessen Logik überhaupt einen Anspruch auf eine Rente begründen. ${ }^{105}$ Dies ist, wie die folgenden Ausführungen verdeutlichen, nicht zwingend

104 Theoretisch wäre selbstverständlich auch hier eine Lösung in Form gestaffelter Fixbeiträge möglich, doch würde sich eine derartige Ausgestaltung dann endgültig nicht mehr von einer nach Version 3 unterscheiden, so dass auf eine Lebensstandardabsicherung mit Hilfe gestaffelter Fixbeiträge in der Aktivphase im weiteren nicht eingegangen wird.

${ }^{105}$ Auch im Folgenden wird ausschließlich die ,Alterssicherungsfunktion' einer Rentenversicherung betrachtet. 
der Fall. Daran anschließend ist zu untersuchen, inwieweit es der Übergang von der ,grundsätzlichen Betrachtung' zur Untersuchung der konkreten Ausgestaltung eines institutionalisierten Rentenversicherungssystems im Umlageverfahren sinnvoll erscheinen lässt, dennoch einen funktionalen Zusammenhang zwischen Beitragszahlung einerseits und Rentenanspruch andererseits zu implementieren. Die Thematisierung erfolgt erst an dieser Stelle, und nicht vor der Untersuchung der ersten beiden Versionen, da Teile der Argumentation einen stärkeren Bezug zu Ausgestaltungselementen, wie sie für die Versionen 3 und 4 typisch sind, haben.

\subsection{Betrachtung eines, naturwüchsigen' Umlageverfahrens}

Der Grundlogik eines ,Generationenvertrags' entspricht, dass die Mitglieder der Aktivgeneration einerseits ihre Elterngeneration versorgen, andererseits eine nachfolgende Generation erziehen. Die Renten können als Erträge des von Eltern gebildeten Humankapitals angesehen werden. Folglich begründen die Beitragszahlungen der Aktiven keinen Anspruch auf den eigenen Erhalt einer Altersrente. Sie begleichen eine ,Schuld' (gegenüber der Elterngeneration), und dienen nicht dem Aufbau einer ,Forderungsposition' (gegenüber den eigenen Kindern). Diesen Zusammenhang verdeutlicht auch die Grundform einer Alterssicherung im Umlageverfahren: die Absicherung der Mitglieder einer Großfamilie. ${ }^{106}$ Die Unterstützung der Elterngeneration durch ihre Kinder in der Zeit, in der sie sich nicht (mehr) selbst versorgen kann, hilft den Letzteren für ihre Altersphase nicht unmittelbar - ihre Eltern sind zur Unterstützung in dieser Phase aus naheliegenden Gründen nicht mehr in der Lage. Die Versorgung der Eltern ist also eher, (moralische) Pflicht' als ,Investition'. Es kann allerdings argumentiert werden, durch diese Versorgungsleistung würden die jetzt erwachsenen Kinder ihren eigenen Kindern ein ,gutes Vorbild' geben - und dadurch die Chance auf eine eigene Versorgung in ihrer Altersphase durch ihre Kinder erhöhen. In dieser Hinsicht kann die Unterstützung der Alten also auch dann sinnvoll sein, wenn diesen gegenüber keine ,Verpflichtung' verspürt wird: Sie ist dann als Aufwand zur Erhaltung einer Tradition zu verstehen, von deren Fortführung durch die Kinder die eigenen Lebenschancen im Alter abhängen (können). Die Unterstützung der Alten wäre dann eine Investition in ,Familientradition' oder, Sozialkapital'.

Das eigene Alterseinkommen kann nur über die Aufzucht einer nachfolgenden Generation, die ihre dann alten Eltern unterstützt, gesichert werden. Aus produktionstechnischer Sicht ist diese Argumentation auch problemlos einsichtig: Ist das ,Rentnerdasein' ökonomisch durch Konsum jenseits der eigenen Produktionsmöglichkeiten charakterisiert, so hat die Altersabsicherung die Existenz von ,Akti-

${ }^{106}$ Für eine schematische Darstellung der ,Finanzbeziehungen' zum intertemporalen Einkommenstransfer zwischen Mitgliedern unterschiedlicher Generationen in Abhängigkeit von der Form des Altersabsicherung vgl. EEKHOFF (1997), S. 41 - 45. 
ven', die periodenbezogen mehr produzieren als sie konsumieren, zur notwendigen Bedingung. Eine eigenständige - ohne Bezug auf eine Aktivgeneration erfolgende - Sicherung der Rentner ist folglich nicht möglich, und zwar unabhängig von der gewählten Form der Altersvorsorge.

\subsection{Betrachtung eines familienübergreifenden kollektiven Umlageverfahrens}

Die obigen Ausführungen haben verdeutlicht, dass in der Grundlogik des Umlageverfahrens Beitragszahlungen keinen späteren Rentenanspruch begründen. Ursächlich für einen solchen kann nur das Aufziehen einer nachfolgenden Generation sein, die in ihrer Aktivzeit die Versorgung ihrer (dann alten) Elterngeneration übernimmt. ${ }^{107}$ Für eine innerfamiliär ausgestaltete Absicherung ergeben sich aus der Grundlogik auch keine sonderlichen Probleme, wenn eine entsprechende emotionale Bindung der Mitglieder der verschiedenen Generationen besteht. ${ }^{108}$ Insbesondere kann dann auch von einer gewissen Bereitschaft einzelner Familienmitglieder zur Tragung einer größeren Versorgungslast für den Fall, dass sie - aus welchen konkreten Gründen auch immer - in einer besseren, Versorgungsposition' als andere Familienmitglieder sind, ausgegangen werden. Zur intrinsischen Motivation kommt im Familienverbund auch die besonders gute Möglichkeit zur Kontrolle hinzu, inwieweit ein einzelnes Familienmitglied einen allgemein als angemessen empfundenen Beitrag zur Lastentragung leistet, wobei als Sanktionsmechanismus die Möglichkeit einer sozialen Ausgrenzung von Verweigerern (extrinsische Motivation) besteht. Ferner sieht der die Unterstützung Leistende unmittel-

${ }^{107}$ Für die Bedeutung von Kindern als ,Mittel' des intrafamiliären Kaufkrafttransfers in die Altersphase der Eltern vgl. NERLOVE et al. (1987), Kap. 9.

${ }^{108}$ Wobei hierbei das Problem einer Ausbeutung gutmütiger Familienmitglieder durch kalkulierend eigennützig agierende Akteure nicht ganz auszuschließen ist - es mag in der Familie schwer fallen, ,Schmarotzer' zu bestrafen. Zwar bietet unter bestimmten Bedingungen die Existenz eines altruistischen Familienoberhauptes die Möglichkeit, dass sich alle Mitglieder der Gesamtfamilie so verhalten, als maximierten sie eine Familiennutzenfunktion (das ,Rottenkid'-Theorem BECKERs (1974); vgl. auch BECKER (1976)). Doch sind die Bedingungen, unter denen eine Familiennutzenfunktion unterstellt werden kann, vergleichsweise restriktiv (BECKER (1977) und HIRSHLEIFER (1977)): Insbesondere muss der Altruist als ,Letzter' - im Wissen um das Verhalten der anderen Familienmitglieder, also bei Möglichkeit der Kontrolle entscheiden können. Dies ist bei Mittellosigkeit im Alter aber gerade nicht der Fall. Zu den transaktionskostenmäßigen Vor- und Nachteilen einer ,familieninternen Lösung' vgl. POLLAK (1985). Zur allgemeinen Möglichkeit einer Ausnutzung von Hilfsbereitschaft, oder auch nur einer Verhaltensänderung, die die Notwendigkeit des Hilfeempfangs deutlich erhöht, wenn auf Seiten des potenziell Unterstützten die Kosten der Hilfeleistung auf Seiten des Helfers nicht berlicksichtigt werden (das ,Samariter-Dilemma'), vgl. BERNHEIM und STARK (1988), BERTHOLD (1992) sowie BRUCE und WALDMAN (1990) mit weiteren Literaturverweisen. 
bar die positiven Wirkungen seiner, Entbehrungen'. All dies hat zur Folge, dass von einer im Normalfall ziemlich reibungslos verlaufenden Aufbringung der Mittel zur Unterstützung hilfsbedürftiger Familienmitglieder ausgegangen werden kann (wenn die insgesamt zur Verfügung stehenden Mittel eine solche Versorgung erlauben).

Bei einem ,kollektiven Umlageverfahren' zur Alterssicherung kann nicht mehr ohne weiteres die Wirksamkeit der gerade genannten Mechanismen, die einen stabilen Fluss der Transfers zwischen den einzelnen Mitgliedern der verschiedenen Generationen sicherstellen können, unterstellt werden. Insbesondere ist die Anonymisierung innerhalb der , Institution GRV' mit allen ihren Wirkungen auf die Zahlungsbereitschaft (aber auch die Interessen der Rentenbezieher) zu berücksichtigen: Da die eigenen (Beitrags-)Zahlungen nicht mehr in einem unmittelbaren Zusammenhang mit den von den eigenen alten Verwandten erhaltenen Renten stehen, wird die individuelle Bereitschaft zur Zahlung wesentlich geringer sein, da das Wohlergehen von Verwandten regelmäßig eine höhere Gewichtung in der Nutzenfunktion der (potenziellen) Beitragszahler hat als das Wohlergehen Fremder. Die nutzenmäßige Schlechterstellung der Zahler infolge der zu leistenden Zahlungen ist in einem anonymen System also grundsätzlich höher als in einem familiären. Die - vermutlich angeborenen - familiären Pflegetriebe und eine genetisch verankerte Kleingruppenmoral stützen dies. Aus einer solchen Sicht lohnt sich die Vermeidung von Beitragszahlungen, die doch im wesentlichen Fremden zugute kommen. ${ }^{109,110} \mathrm{Da}$ ihre Renten in einem vollkommen anonymen System nicht von den Beitragszahlungen ihrer Kinder abhängen, gibt es obendrein von Seiten der Alten keinen moralischen Druck hin zu einer (korrekten) Beitragsleistung; dies gilt um so mehr, da mit sinkenden Zahlungen an die GRV die Möglichkeit zu einer innerfamiliären Unterstützung derjenigen steigt, die einem selbst in der Kinder- und Jugendzeit geholfen haben.

Die innerfamiliäre Kontrolle versagt auch in einer anderen Richtung: Da das, was die ,Alten' konsumieren, nicht mehr unmittelbar von ihren eigenen Nachkommen aufgebracht werden muss, sondern von der anonymen Gruppe der Beitragszahler, also nicht automatisch Konsumverzicht auf Seiten der Kinder zur Voraussetzung

109 Eine gänzlich andere Sichtweise der menschlichen Natur hat GLOMBIK (1995, S. 34), der in einer Analyse der Probleme der Rentenversicherung feststellt: „Zwar werden die Beiträge zur Rentenversicherung steigen - bis knapp unter dreißig Prozent -, aber die Einkommen werden auch anwachsen, und für Existenz und Sicherheit im Alter wird man gewiss bereit sein, mehr zu zahlen".

${ }^{110}$ Derselbe Mechanismus tendiert bekanntlich bei Versicherungen dazu, aus Menschen, die einzelne Personen nie betrügen würden, Versicherungsbetrüger zu machen, da sich der Schaden auf die Gesamtheit der Versicherten in individuell nicht spürbarer Weise verteilt. 
hat, besteht die Gefahr einer ,Überforderung' der Aktivgeneration durch die Rentnergeneration: Eine sonst unmittelbar existente Hemmschwelle zur Vermeidung von ,Maßlosigkeit' auf Seiten der Rentner wird aufgehoben. ${ }^{111}$

Die gerade geschilderte Konstellation fördert für ein anonymes System ${ }^{112}$ eine Tendenz zur Vermeidung von Beitragszahlungen an eine Rentenversicherung, wenn die Beitragszahlungen für ein repräsentatives Mitglied der Aktivgeneration ausschließlich einen ,Steuercharakter' haben. Gibt es Möglichkeiten einer Vermeidung der Beitragszahlung, so ist deren Wahrnehmung folglich attraktiv. Um eine ,massenhafte' Flucht aus der Rentenversicherung zu verhindern, bedarf es mithin wirksamer Kontrollaktivitäten. Eine Flucht aus der Rentenversicherung lässt sich durch ein Schließen von ,Schlupflöchern' verringern. Doch auch dann bleibt - neben der Festlegung des allokativ effizienten Maßes an Kontrolle - noch ein weiteres Problem: Wie sollen Renten- und Beitragshöhe ausgestaltet werden? Wenn die Höhe der Rente der Eltern unabhängig von der Höhe der Beitragszahlungen der Kinder ist, dann ergeben sich - bei interindividuellen Einkommensunterschieden - Fälle, in denen die Kinder zusammen erheblich mehr Rentenbeiträge zahlen, als ihre Eltern erhalten. Es gibt also weiterhin (interfamiliäre) Einkommensumverteilungen.

Ferner ist zu beachten, dass bei einer Beitragssatz-Ausgestaltung der Rentenversicherung (Version 3) - wie später noch deutlich werden wird - eine mögliche Reaktion der (potenziellen) Eltern nicht ausschließlich in Form einer geringen Kinderzahl erfolgt, wie sie im Fixbeitrags-Ansatz (Version 1) anzutreffen ist, sondern auch in Form einer vergleichsweise zu geringen Investition in das Humankapital der jeweiligen Kinder. Da nämlich alle Rentner vom besonders hohen Einkommen eines Aktiven profitieren, obwohl die Kosten der Humankapitalbildung in besonderer Weise von den Eltern getragen werden, gibt es einen Fehlanreiz hin zu einer zu geringen ,Humankapitalinvestition' pro Kind. In einer Ausgestaltung der RV nach Version 1 (oder 2) erfolgt hingegen keine Beteiligung der Gesellschaft an den Erträgen hoher Pro-Kopf-Humankapitalinvestitionen der Eltern. Die höheren Einkommen der Kinder infolge des ,Investitionsverhaltens' ihrer Eltern

${ }^{111}$ Das Bestehen - genauer: die Beseitigung - dieser Hemmschwelle wird von bestimmten politischen Kreisen aus dem Umfeld der gegenwärtigen Regierung allerdings auch als Argument zugunsten einer Einfuhrung von Mindestrenten vorgetragen: Es gebe ,verschämte Altersarmut die (partiell) daraus resultiere, dass arme Eltern sich aus Sorge, die Sozialhilfe könne zu einem Rückgriff auf das Einkommen und Vermögen ihrer Kinder führen, nicht an die Sozialhilfe wenden würden. Dies müsse zukünftig durch Änderungen in der Ausgestaltung der Rentenversicherung vermieden werden.

${ }^{112}$ Hier haben die Beitragszahlungen der Kinder auch keinen Einfluss auf die Höhe der Rente ihrer Eltern. 
verbleiben dort als Residualgröße vollständig beim Kind (und somit in der Familie). Ist die Einkommenshöhe des Kindes, die über seine Beiträge zur RV bestimmt, nicht nur Folge von ,Zufälligkeiten' etc., sondern (auch) endogen bestimmt, so diskriminiert eine partielle Sozialisierung des Ertrags von Humankapital also dessen Bildung. ${ }^{113}$

Zwar lassen sich die höheren Beitragsleistungen eines ,Besserverdienenden', denen keine höhere(n) Rente(n) seiner Eltern gegenübersteht, auch als implizite Beiträge zu einer Versicherung auffassen: Die Versichertengemeinschaft gewährt seinen Eltern eine Rente in der gegebenen Höhe auch dann, wenn er infolge eines geringen Einkommens zu einer nennenswerten Mitfinanzierung der Rente nicht in der Lage ist. Insoweit ist es auch bei einem überdurchschnittlichen Einkommen angemessen, dass er für diese Absicherung der Eltern ex post eine Versicherungsprämie in Form eines überdurchschnittlichen Rentenbeitrags zahlt. Doch dies ändert nichts an der individuellen Rationalität einer Beitragsvermeidung. ${ }^{114,115}$ Schließlich setzt jede institutionalisierte Alterssicherung - infolge der mit ihr untrennbar verbundenen ,Kollektivgutillusion' - allokative Fehlanreize. ${ }^{116}$

\subsubsection{3. ,Abgabewiderstände' gegen Rentenversicherungsbeiträge?}

Die gerade bezeichneten allokativen Fehlanreize einer institutionalisierten Alterssicherung sollen zum Anlass genommen werden, kurz auf die Fähigkeit des Rentenversicherungssystems zur Aufrechterhaltung der ,Rentensicherheit' einzugehen. Sehen die Versicherten die Beitragsleistungen als ,belastend' an, kann es

113 Dazu, dass schon die Existenz eines Rentenversicherungssystems den individuellen Anreiz zu Investitionen in das Humankapital verringert, vgl. Abschnitt 2.5.1.

114 Insoweit ist die Feststellung, „[d]ie während der Erwerbsphase zu leistenden Beiträge an die Alterssicherung ... [seien] dagegen als reine Rückzahlungen der zuvor empfangenen Vorleistungen einzustufen und .. [trügen] für sich genommen nichts zur eigenen Altersvorsorge bei" (WERDING (1998), S. 236) zwar per se nicht falsch, aber eben als Ausgestaltungsvorschlag fur eine Rentenversicherung bei endogener Entscheidung über den Arbeitseinsatz kritisch zu hinterfragen.

${ }^{115}$ Das geschilderte Problem einer Vermeidung der Beitragszahlung kann es auch bei einer Ausgestaltung der Rentenversicherung nach Version 1 oder Version 2 geben, wenn die Möglichkeit einer Befreiung von einer Pflicht zur Beitragszahlung besteht. Die kumulierte Beitragszahlung mag eher Einfluss auf die Entscheidung hin zur Realisation einer ,Totalverweigerung' (Auswanderung) haben, während die Beitragsbelastung marginale Einkommen (die es in Version 1 und 2 nicht, wohl aber in Version 3 gibt) eher Einfluss auf eine marginale Arbeitsangebotsresp. Einkommenserzielungsentscheidung hat.

116 Vgl. HACKMANN (1990), S. 69f. 
zu Verhaltensänderungen kommen, die die Fähigkeit des Versicherungssystems zur Aufbringung der benötigten Finanzmittel beeinträchtigen. Dies ist insbesondere dann der Fall, wenn - wie im gleich zu thematisierenden Beitragssatzverfahren - die Rentenversicherungsbeiträge als Abzug vom (rentenversicherungspflichtigen) Einkommen erhoben werden. Hier kann (wird) es Rückwirkungen des Beitragssatzes auf das (rentenversicherungspflichtige) Einkommen geben. Vergleichbar mit der Erhebung einer Steuer, die die Wirtschaftssubjekte - typischerweise zu einer Verringerung der besteuerten Aktivität verleitet, können höhere Beitragssätze zur Rentenversicherung auf das Einkommen die Bereitschaft der Mitglieder der Aktivgeneration zur Einkommenserzielung senken, so dass womöglich bei steigenden Abgabesätzen sogar ein geringeres Beitragsaufkommen realisiert wird. Es gibt insoweit eine gewisse Ähnlichkeit zu der aus der Diskussion um die Laffer-Curve bekannte Problematik einer Einkommensbesteuerung. Auf den Sinn bzw. Unsinn der Laffer-Curve bzw. den aus dieser politisch resp. propagandistisch gezogenen Schlussfolgerungen für bestehende Ausgestaltungen des Steuersystems ist an dieser Stelle nicht weiter einzugehen. Doch auch bei Skepsis gegenüber Begründungen von Steuersatzsenkungen mit Hilfe der Laffer-Curve (z.B. infolge nur schwer zu bestimmender Bereiche, in denen Steuersatzsenkungen zu Mehreinnahmen führen), ist von einer Bedeutung des dem Konstrukt zugrunde liegenden Sachverhaltes einer Reagibilität der Bemessungsgrundlage auf Steuersatzvariationen für den Bereich der Rentenversicherung auszugehen, wenn (bzw. insoweit) die Beiträge einen ,Steuercharakter' haben.

Damit die Vermeidung von Beitragszahlungen und die damit einhergehende Verringerung der Bemessungsgrundlage der Beitragszahlungen zu einem Problem wird, muss es Gründe für die Versicherten geben, die (marginale) Beitragszahlung als ,unattraktiv' anzusehen. Für diese Beurteilung ist die von den Versicherten erwartete (individuelle) Rendite ihrer marginalen Beitragszahlungen bedeutsam: Eine extreme Anreizkonstellation zugunsten einer Beitragsvermeidung liegt vor, wenn zwischen der Höhe der individuellen Beitragszahlung in der Aktivzeit und der Höhe der individuellen Rente im Alter kein positiver Zusammenhang besteht. Der Rentenbeitrag hat in diesem Fall reinen ,Steuercharakter', da eine Leistung ohne spezifische Gegenleistung vorliegt. ${ }^{117}$ Und auch sonst ist der Anreiz zur Vermeidung einer Beitragszahlung umso höher, je niedriger die erwartete Verzinsung im Vergleich zu einer Alternativanlage ist.

${ }^{117}$ Insoweit sind z.B. die in letzter Zeit häufiger auftauchenden Gedankenspiele über eine Reduktion der Rentenversicherung auf die Sicherung einer (Einheits-)Grundrente potenziell äußerst problematisch: Bei einem Übergang zu einer Grundrente könnte der Wert marginaler Beitragszahlungen in der Aktivzeit auf null sinken! 
Ein weiterer Grund für Vermeidung von Beitragszahlungen kann sein, dass die Zahlungsverpflichtung in der Aktivzeit die individuell gewünschte intertemporale Aufteilung des Lebenskonsums behindert (subjektive Wahrnehmung einer zu hohen Ersparnis in der Aktivzeit). Die beiden gerade genannten Begründungen für eine Beitragsvermeidung gehen fließend ineinander über, wenn ein System periodisierter Rentenzahlungen betrachtet wird, und der Versicherte für sich selbst eine eher unterdurchschnittliche Rentenbezugsdauer erwartet (er meint für die Rentenversicherung ein ,gutes' Risiko darzustellen, und somit zur Subventionierung der ,schlechten Risiken' herangezogen zu werden).

Durch das Ausweichen in nicht-versicherungspflichtige Aktivitäten kann er dann seine finanzielle Unterstützung schlechter Risiken vermindern. Dies kann auf verschiedene Weise geschehen. Eine besondere Bedeutung hat hierbei das Ausweichen auf nicht-abgabepflichtige Aktivitäten (so z.B. Aktivitäten mit Einkommen, das nicht der Rentenabgabe unterliegt, aber auch Freizeitkonsum und Do-it-yourself), die noch dazu um so größer ist, je einfacher ein solches Ausweichen ist. Eine Ausweichreaktion setzt die Möglichkeit einer Vermeidung von Beitragszahlungen voraus. Bei den betrachteten Versionen 1 und 2 ist eine solche Möglichkeit infolge des individuellen Pauschalcharakters der Beitragszahlungen nicht gegeben, wohl hingegen (insbesondere bei ,Nicht-Belastung' von Freizeit) bei Version 3.

Zusammenfassend lassen sich Ausweichreaktionen darauf zurückführen, dass eine Einzahlung in die Rentenversicherung nicht als ,gute Investition' aufgefasst wird. Die Individuen sehen Alternativanlagen, für die sie einen höheren (Real-)Zins erwarten. ${ }^{118}$ Die Rentenversicherung hat insoweit einen als ,inhärent destabil' zu kennzeichnenden Charakter: Kommt es zu einer Verringerung der Bemessungsgrundlage infolge der Vermeidung rentenversicherungspflichtiger Aktivitäten auf Seiten der Versicherten, so senkt dies entweder - bei unverändertem Beitragssatz - die Rentenhöhe der gegenwärtigen Rentnergeneration, und bestätigt somit das Misstrauen der heutigen Aktiven in die ,Sicherheit' ihrer späteren Rente, was den Drang weg von versicherungspflichtigen Aktivitäten eher verstärken dürfte, oder aber es kommt zu Beitragssatzsteigerungen. Da diesen aber auf Dauer gewisse Grenzen gesetzt sind, ist es aus Sicht derjenigen, die heute höhere Beitragszahlungen leisten sollen, nicht sicher, dass eines Tages auch zur Sicherung ihrer Renten(höhe) der Beitragssatz womöglich weiter erhöht wird. Wenn die heutige

118 So schätzt SCHNABEL (1998a) die reale Verzinsung der Beitragszahlungen zur deutschen Gesetzlichen Rentenversicherung für im Jahre 1980 Geborene auf durchschnittlich 0 bis 1 v.H. und kommt zu dem Ergebnis: „, The implicit tax for the youngest of the cohorts will rise to twothirds of the pension contributions, which translates into a tax rate of 18 percent of the gross wage bill" (ebd., S. 3). 
Steigerung der Beitragszahlung aber nur den heutigen Alten zugute kommt, und eine entsprechende Sicherung der Rendite der eigenen Beitragszahlungen nicht gegeben ist, so erscheint eine individuelle Absicherung außerhalb des Rentenversicherungssystems - mit einhergehender Verminderung der Beitragszahlungen zur GRV - um so attraktiver. Aus dem System der zwangsweisen Rentenversicherung heraus gibt es keine Gegenkräfte gegen solche Tendenzen. Stabilsierungstendenzen müssen von den äußeren Rahmenbedingungen kommen.

Die gerade verdeutlichte (mögliche) Instabilität einer umlagefinanzierten Rentenversicherung mit einer Version 3 entsprechenden Ausgestaltung hat verschiedene bedeutsame Folgen: Zum einen gefährdet jede Diskussion über eine weitere Verringerung der Rendite marginaler Beitragszahlungen, wie sie insbesondere im Falle der Umgestaltung der Rentenversicherung hin zu einer Grundsicherung im Alter in Form einer (Einheits-)Grundrente vorliegt, die Finanzierungsbasis der gegenwärtigen Ausgestaltung. Zum anderen ist eine inhärente Instabilität eines so wichtigen wirtschaftlichen Sicherungssystems, wie es die Rentenversicherung darstellt, problematisch: Verschlechterungen in den Umgebungsbedingungen werden nicht systemimmanent aufgefangen bzw. zumindest abgefedert, sondern es besteht die Gefahr einer Verstärkung der Probleme. Diese Überlegungen verdeutlichen aber auch, dass eine ,automatische Selbststeuerung' einer nach Version 3 ausgestalteten Rentenversicherung nicht zu erwarten ist. Gerade in Krisenzeiten scheinen politische Eingriffe zur Vermeidung einer (weiteren) Selbstdestabilisierung unvermeidbar. Sie sind allerdings mit der Gefahr verbunden, dass durch die Eingriffe das Vertrauen in das System weiter gestört wird. Die Anforderungen an eine erfolgreiche externe Steuerung der Rentenversicherung sind mithin hoch.

Existiert keine automatische (,systemimmanente') Stabilisation, bedarf es einer ,äußeren' Stabilisation. Die Rolle des Stabilisators übernimmt im Rahmen einer Gesetzlichen Rentenversicherung - wie der Name schon sagt -, der Staat, der die Beiträge zur GRV zwangsweise eintreibt (bzw. eintreiben lässt). Insoweit das Vermeiden der Beitragszahlungen also mit anderweitigen Nachteilen verbunden ist - insbesondere strafrechtlicher Verfolgung, aber auch faktischen Berufsverboten -, wird eine gewisse ,Bereitschaft' zur Zahlung der Beiträge durchgesetzt.

Staatlicher Zwang zur Stabilisierung einer im Umlageverfahren organisierten (existierenden) Rentenversicherung ist für die gegenwärtigen - zu einer Beitragszahlung gezwungenen - Aktiven allerdings nicht ausschließlich negativ: Es ist offensichtlich, dass die Existenz einer nachfolgenden - der GRV beitretenden Aktivgeneration zur Sicherung der Rentenzahlungen an die jeweiligen Rentner 
notwendig ist. ${ }^{119}$ Wenn aber - infolge eines sinkenden Umfangs der Aktivgeneration bei abnehmender wirtschaftlicher Dynamik - die ,Sicherheit' der Rente in einiger Zeit nicht mehr gegeben zu sein scheint, so könnte im Sinne einer rückwärtigen Induktion unterstellt werden, dass auch vorhergehende Generationen nur durch ,Zwang' zum Beitritt in die GRV bewegt werden können. Und hier wirkt der Staat durch die Erhöhung der Kosten des Nicht-Beitritts (sei es durch strafrechtliche Verfolgung, sei es in der Form von ,Berufsverboten' etc.) stabilisierend, nimmt durch die höheren Vermeidungskosten doch die Attraktivität eines Beitritts zur GRV relativ zu einer anderweitigen Alterssicherung zu. Die ,kritische Renditedifferenz', jenseits derer die Vermeidung eines GRV-Beitritts für große Teile der Aktivbevölkerung attraktiv ist, wird durch staatlichen Zwang mithin erhöht. Der Einsatz des Staates für die Eintreibung des Beitragsaufkommens zur Sicherung der gegenwärtigen Rentenzahlungen kann als eine Art Selbstbindung verstanden werden: Der Staat zeigt, dass er einen Zusammenbruch des Rentenversicherungssystems mit weitgehenden Maßnahmen zu verhindern trachtet, was das Vertrauen in die Sicherheit einer späteren Rentenzahlung als ,Gegenleistung' für die Beitragszahlungen in der Aktivzeit erhöht. Und eine konsequente Beitragsaufbringung auch in zukünftigen Perioden steigert die erwartete Rente im Alter, und fördert so die Bereitschaft zur Beitragszahlung in der Gegenwart.

\subsubsection{Probleme der, Renditemessung' für ein Umlageverfahren}

(Mit-)Entscheidend für die individuelle Sinnhaftigkeit (und Intensität) einer Vermeidung von Beitragszahlungen zur Rentenversicherung ist die Differenz zwischen der (erwarteten) Rendite von Beitragszahlungen zur Rentenversicherung und der Rendite von Alternativanlagen. ${ }^{120}$ Eine vergleichsweise niedrige (erwartete) Rendite der Zahlungen zur Rentenversicherung steigert die Attraktivität einer Vermeidung von Beitragszahlungen. Insoweit die jeweilige Beitragszahlung diejenige übersteigt, die der Versicherte bei seinen Erwartungen bezüglich der Rendite seiner Einzahlungen (zuzüglich einem ,Zuschlag' für den Versicherungsschutz) freiwillig leisten würde, sind mit dem Pflichtbeitrag also negative Anreizwirkungen verbunden.

119 Auch beim KDV bedarf es - wie bereits mehrfach betont - einer nachfolgenden Generation. Da die Alten aber Aktiva abzugeben haben, gibt es eher die Möglichkeit, die Rente durch den Verkauf an Ausländer oder nicht der GRV Beigetretene in Teilen zu sichern. Zu den Problemen einer Rentenversicherung ohne nachfolgende Generation vgl. SAMUELSON (1958), S. $474 f$.

${ }^{120}$ Ein weiterer Grund für eine Vermeidung von Beitragszahlungen zur Rentenversicherung können Abweichungen des, intertemporalen Belastungsprofils' vom gewünschten Profil sein also z.B. eine als zu hoch empfundene Belastung in Zeiten hohen Finanzbedarfs. 
Das Problem bei dieser Argumentation ist die Bestimmung der zu vergleichenden (erwarteten) Renditen unterschiedlicher Anlageformen. So stellt sich die Frage nach der Vergleichbarkeit von Einzahlungen in die GRV mit ,normaler' Ersparnisbildung. Zwar wird in der umlagefinanzierten Rentenversicherung kein Geldbzw. Sachvermögen akkumuliert, auf das später bei Bedarf zurückgegriffen werden kann. Dafür kommt es jedoch zu einem Aufbau von Ansprüchen gegenüber der nachfolgenden Generation. Die Abschätzung der Rendite von Beitragszahlungen in Abhängigkeit von den Umgebungsbedingungen (zu denen auch die politischen Steuerungsmechanismen gehören) sowie individueller Charakteristika des Versicherten ist ex ante kaum möglich. ${ }^{121}$

Aufgrund der Konstruktion ist es in einem UV zwar möglich, einem repräsentativen Versicherten (einer konkreten Generation) für seine Einzahlungen eine bestimmte ,Rendite' zuzusichern und somit für diesen Versicherten die Einzahlung in die umlagefinanzierte RV in die Nähe anderer Kapitalanlagen zu rücken - doch eine derartige ,Zusage' kann systembedingt nicht für alle Versicherten aller Generationen gegeben werden; dies gilt selbst für den Fall, dass der Fortbestand des Rentenversicherungssystems in seiner grundsätzlichen Ausgestaltung unterstellt wird, was per se schon eine weitreichende Annahme ist. ${ }^{122}$ Die Schwierigkeiten einer ,Renditezusage' resultieren vor allem daraus, dass die Summe des rentenversicherungspflichtigen Einkommens in den nachfolgenden Perioden zum Zeitpunkt der Beitragszahlung unbekannt ist. Die Zusage einer bestimmten ,Rendite' auf die Einzahlungen würde mithin nichts anderes als die Zusage darstellen, die Mitglieder der nachfolgenden Generation während ihrer Aktivphase so zu belasten, dass ihre Beitragszahlungen ausreichen, um das der Rentnergeneration in ihrer Aktivzeit gegebene Renditeversprechen einzuhalten. Mithin würde der Beitragssatz zu einer Variablen, die ausschließlich mit dem Ziel der Befriedigung der Ansprüche der jeweiligen Rentnergeneration variiert würde. In einer Zeit mit einer steigenden Summe der beitragspflichtigen Einkommen ist eine solche Renditezusage allerdings unproblematisch, ihre Einhaltung mag sogar mit sinkenden Beitragssätzen der Aktivgeneration verbunden sein kann. Entwickelt sich die Einkommenssumme jedoch weniger dynamisch, als es zu einer Befriedigung der zugesagten Rendite bei konstantem Beitragssatz nötig wäre, so muss der Beitragssatz erhöht werden. Auf diese Art und Weise lässt sich die versprochene Rendite zunächst sichern. Das Problem besteht aber darin, dass durch die erhöhten Beiträge zur Befriedigung der Alten die Aktivgeneration - im Rahmen der vorgestellten Logik -

${ }^{121}$ Für Abschätzungen zur ,internen Rendite' von Beitragszahlungen zur GRV für repräsentative Mitglieder unterschiedlicher Geburtsjahrgänge (unter der Annahme geringer Änderungen der Umgebungsbedingungen) vgl. SCHNABEL (1998a), ders. (1998b), RAFFELHÜSCHEN (1998).

Vgl. SPREMANN (1984), SPREMANN (1986), FAMULLA und SPREMANN (1980). 
ihrerseits höhere Rentenansprüche für ihre eigene Altersphase erwirbt. Eine begrenzte Zeit ist also bei nicht hinreichend dynamischer Einkommensentwicklung die Sicherung der Rendite durch Beitragssatzsteigerungen möglich - aber eben nicht dauerhaft, da es einerseits eine natürliche Obergrenze der Ansprüche der Rentner gibt (nämlich das gesamte Volkseinkommen), und andererseits eine wohl deutlich niedriger liegende - ,praktische Grenze', ab derer sich der Widerstand gegen Beitragszahlungen derart erhöht, dass Kontroll- und Verwaltungskosten die zusätzlichen Einnahmen übersteigen oder - wenn die Kontrolle gelänge - der Leistungswille der Aktivgeneration gänzlich schwindet. Dies ist spätestens dann der Fall, wenn die erforderlichen Leistungszusagen das künftige Sozialprodukt überausschöpfen. Es gibt also das Problem, wie die nachfolgende Generation zur Erfüllung des von den ,Alten' eingeforderten Renditeversprechens bewegt werden kann.

Ähnliche Problemlagen gibt es aber auch bei einem Kapitaldeckungsverfahren. Bei dessen Diskussion (vgl. 2.6) wurde schon auf mögliche Rückwirkungen demographischer Verschiebungen auf die Preise von Kapitalgütern verwiesen, ebenso auf die - u.U. aber riskante - Möglichkeit von Auslandsanlagen, die einem relativ kleinen offenen Land, das von Ländern mit Kapitalmangel umgeben ist, eine längerfristige Erzielung recht hoher Renditen erlaubt. Ebenso sind Änderungen der Nachfrage nach Kapitalgütern durch die Mitglieder der Aktivgeneration (z.B. infolge veränderten Pro-Kopf-Einkommens, veränderter Neigung zum freiwilligen Sparen etc.) als mögliche Ursache von Preisverschiebungen zu bedenken. Schon diese Preisstruktureffekte und Änderungen der Verwertungsmöglichkeiten für Kapital verhindern eine längerfristige Zusage einer ,realen' Rendite auch beim Kapitaldeckungsverfahren.

Entscheidend für das Verhalten der jeweiligen (potenziellen) Beitragszahler ist im Übrigen nicht die ,allgemeine' Rendite von Beitragszahlungen, sondern die von ihm erwartete individuelle Rendite. Diese hängt (in einem realitätsnahen Analyserahmen) von einer Vielzahl individueller Einflussfaktoren ab. Die Berücksichtigung dieser individuellen Umstände macht einen individuell aussagefähigen Renditevergleich vollends unmöglich. ${ }^{123}$

${ }^{123}$ Hierbei ist allerdings zu beachten, dass viele der in der heutigen Ausgestaltung der umlagefinanzierten Rentenversicherung in Deutschland anzutreffenden Umverteilungsmechanismen nicht unmittelbare Folge des Umlageverfahrens sind; entsprechende Mechanismen könnten mithin auch in einer (zwangsweisen) kapitalgedeckten Rentenversicherung implementiert werden. 


\subsubsection{Besonderheiten der individuellen Rentensicherung durch Beitragsleistungen bei einem Beitragssatz-Umlageverfahren}

Infolge der schon beschriebenen allokativen Fehlanreize, die ein kollektives Sicherungsverfahren, bei dem die Beitragszahlung mit der Höhe des Einkommens steigt, durch Beitragszahlungen jedoch kein eigener Rentenanspruch erworben wird, unvermeidlich setzt (Meidung rentenversicherungspflichtiger Aktivitäten, ,Beitragshinterziehung' etc.), erscheint es angemessen, nach Möglichkeiten einer Verminderung dieser Anreizprobleme zu suchen. Der einfachste Weg einer Vermeidung von Fehlanreizen, nämlich der Übergang zu einer streng familiären $\mathrm{Al}$ terssicherung, hat große Nachteile. Zu nennen ist vor allem die fehlende Absicherung für den Fall von Kinderlosigkeit oder geringer Einkommen der Kinder, die dann nicht zu einer (ausreichenden) Unterstützung der Eltern in ihrer Altersphase in der Lage sind. Die geringe durchschnittliche Familiengröße - auch bei Betrachtung der ,erweiterten Großfamilie' - verringert die innerfamiliäre Möglichkeit einer Tragung von besonderen Risiken der Alterssicherung (so z.B. extremer Langlebigkeit von Familienmitgliedern). ${ }^{124}$ Gilt eine kollektive Alterssicherung deshalb als geboten, ${ }^{125}$ besteht die Aufgabe einer Kinderberücksichtigung darin, externe Effekte des Kinderhabens zu internalisieren. ${ }^{126}$

Wird in der Rentenversicherung ein positiver Zusammenhang zwischen der Höhe der Beitragszahlungen und der Höhe der Versicherungsleistungen hergestellt, sind die allokativen Anreize zu einer Vermeidung der Beitragszahlung geringer. Grundsätzlich sind hierbei zwei verschiedene Verbesserungen der Leistung der

${ }^{124} \mathrm{Ob}$ die Einführung eines Rentenversicherungssystems für einen Rückgang der Familiengröße ursächlich ist, oder aber vor allem Folge eines sich aus der abnehmenden Möglichkeit zur intrafamiliären Alterssicherung ergebenden zunehmenden Interesses weiter Bevölkerungsteile an institutionalisierter Absicherung war, braucht für die folgende Argumentation nicht weiter zu interessieren.

${ }^{125} \mathrm{Ob}$ es nicht andere Verfahrensweisen gibt, die angesichts dieser ,Risiken" den Vorzug verdienen, ist hier nicht systematisch zu prüfen. Wie anfangs ausgefuhrt, wird von der Existenz eines Umlageverfahrens ausgegangen.

${ }^{126}$ Die Feststellung OETERs (1989b, S. 49; 1997, S. 398), auf Grund der Verteilung von Kindern auf die Mitglieder der Aktivgeneration ergebe sich, dass die Kinder eines Tages 30 v.H. ihrer Beiträge an Kinderlose und 20 v.H. an Leute mit nur 1 Kind fließen săhen, hilft hier per se kaum weiter. Wer Kinderlose absichern will, der kommt um Zahlungen auch an Kinderlose nicht herum. Auch nach einem weitgehenden Ausgleich der externen Effekte des Kinderhabens im Rahmen der Rentenversicherung (z.B. durch Beitrags- oder Leistungsstaffelung nach Kinderzahl etc.) werden Zahlungen von Kindern an Kinderlose fließen. OETER selbst (1997, S. 398) schließt letztlich auf die Angemessenheit von „Kinderersatzzahlungen im Rahmen der Rentenversicherung", ohne anzumerken, dass dies nichts an den Zahlungsströmen (auch) von ,Kindern' an Kinderlose (in deren Altersphase) ändert. 
Versicherung mit steigender Beitragsleistung eines Aktiven möglich: Zum einen eine Erhöhung des Rentenanspruchs seiner Eltern, d.h. eine Verringerung des ,Sozialisierungsgrades' der Erträge der Humankapitalinvestitionen, zum anderen eine Steigerung des späteren ,Rentenanspruchs' relativ zu dem von Aktiven mit geringerer Beitragsleistung. ${ }^{127}$

\subsection{Höhere Renten der Eltern infolge höherer Beitragszahlungen ihrer Kinder}

Bei einer ,elternorientierten' Berücksichtigung der Höhe der Beitragszahlung(en) ihrer Kinder steigt die Rente der Eltern (c.p.) mit der Summe der Beitragszahlungen ihrer Kinder in einer gegebenen Periode. Während also gleichmäßige Steigerungen der Einkommen aller Aktiven allen Rentnern gleichermaßen zugute kommen, führen mit einer konstanten Beitragssumme einhergehende Verschiebungen der Einkommensstruktur der Aktiven zu - mit einer konstanten Rentensumme einhergehenden - Änderungen der Einkommenspositionen innerhalb der Gruppe der Rentner.

Vorteil einer solchen Ausgestaltung ist, dass die ,natürliche' Logik eines Umlageverfahrens, nämlich dass eine Generation als ganze nur durch die Bildung von Humankapital ihren Alterskonsum sichern kann, verdeutlicht und der Zusammenhang zwischen individueller Erziehungsleistung und Rentenhöhe auch individuell erfahren wird. Der hergestellte unmittelbare Zusammenhang zwischen Rentenhöhe und Beitragszahlungen der Kinder macht eine ,Flucht' aus der Rentenversicherung auf Seiten der Aktiven weniger attraktiv, hat also sowohl auf Seiten der Eltern (bzw. ihres Anreizes zur Bildung von Humankapital) als auch auf Seiten der Kinder (bzw. ihres Anreizes zu Beitragsleistungen) positive Anreizwirkungen.

Es ist allerdings zu beachten, dass in einer Rentenversicherung, die auch einer Altersabsicherung Kinderloser dient, regelmäßig Teile der Beitragsleistungen der Aktivgeneration an kinderlose Rentner transferiert werden. Da steigende individuelle Beitragszahlungen also nicht ausschließlich zu höheren Renten bei den Eltern derjenigen führen, die diese höheren Beitragsleistungen aufbringen, ist die Vermeidung von Beitragszahlungen für einen Aktiven (und für dessen Familie) jedoch weiterhin individuell attraktiv. Insbesondere nach dem Tod der Mitglieder der Elterngeneration hat eine höhere Beitragszahlung eindeutig einen reinen Steu-

${ }^{127}$ Die Ausfuhrungen hier beziehen sich auf, reine' Ausgestaltungen; Mischformen, bei denen eine höhere Beitragszahlung des Aktiven sowohl die Rente seiner Eltern erhöht als auch seine eigenen Rentenansprüche, sind problemlos denkbar. Auf eine solche Ausgestaltung wird später noch zurückgekommen. Bis auf weiteres werden jedoch die, reinen' Ausgestaltungen betrachtet. 
ercharakter, kann doch definitionsgemäß kein eigenes Familienmitglied dann mehr von dem höheren (potenziellen) Rentenanspruch ,profitieren'.

\subsection{Höhere eigene Renten infolge höherer eigener Beitragszahlungen}

In den weiteren Ausführungen wird von einer Ausgestaltung des BeitragssatzUmlageverfahrens ausgegangen, bei der höhere individuelle Beitragszahlungen in der Aktivzeit spätere höhere eigene Renten induzieren. Als Folge einer solchen Ausgestaltung schlagen sich Einkommensunterschiede in der Aktivzeit in Unterschieden in der Höhe der Renten nieder, tendenziell wird also die relative Einkommensposition innerhalb der Mitglieder der jeweiligen Generation aus der Aktivphase in die Rentenphase übernommen. Auch bei einer solchen Vorgehensweise partizipiert die Rentnergeneration als ganze an Steigerungen der Einkommenssumme der Mitglieder der Aktivgeneration, womit noch nichts über die Aufteilung des zusätzlichen Beitragsaufkommens auf die einzelnen Mitglieder der Rentnergeneration ausgesagt ist.

\subsection{Anreizwirkungen}

Da bei dem hier diskutierten Verfahren höhere Beitragszahlungen in der Aktivphase einen (c.p.) höheren Rentenanspruch in der Altersphase induzieren, wird ein repräsentativer Beitragszahler seine Beiträge zur Rentenversicherung nicht - zumindest nicht gänzlich - als für ihn ,verlorenes Geld' ansehen, was den Anreiz zur Vermeidung von Beitragsleistungen vermindert. Insbesondere erhöht sich (c.p.) die erwartete Rendite der Beitragszahlungen - auch relativ zu Alternativanlagen.

\subsection{Exkurs: Unterschiede und Gemeinsamkeiten von Änderungen des Beitragssatzes und der Bemessungsgrundlage}

Wie bereits erwähnt, gibt es für die Träger der Rentenversicherung zur Steigerung der Beitragseinnahmen zwei ,reine' Strategien: Zum einen kann der Beitragssatz erhöht werden, zum anderen kann, wenn nicht bereits jedes Einkommen der Aktiven einer Periode zur Finanzierung der GRV herangezogen wird, die Beitragsbemessungsgrundlage ausgeweitet werden. Es stellt sich somit die Frage, ob die beiden hier genannten Methoden gleichwertig sind, oder worin eventuelle Unterschiede bestehen.

Eine Verbreiterung der Bemessungsgrundlage hat in einer Gesellschaft, in der die Individuen einer Generation in allen relevanten Parametern übereinstimmen, zwei voneinander zu trennende Auswirkungen: Für schon vorher der Rentenversiche- 
rungsabgabe unterworfenes Einkommen liegt der marginale Beitragssatz zur GRV niedriger als bei einer Steigerung des Beitragsaufkommens über eine Erhöhung des Beitragssatzes. Für die Teile des Einkommens, die infolge der Verbreiterung der Bemessungsgrundlage erstmals der Beitragspflicht unterliegen, steigt hingegen die Grenzbelastung bei dieser Strategie an, während sie bei einer Steigerung des Beitragssatzes ohne damit einhergehende Verbreiterung der Bemessungsgrundlage unverändert (bei null) bleibt. Da die Belastung des marginalen Einkommens für die Anreizwirkungen entscheidend ist, haben die beiden Strategien der Einnahmeerzielung grundsätzlich unterschiedliche Wirkungen. ${ }^{128}$

Für die Betrachtung der Wirkungen einer Verbreiterung der Bemessungsgrundlage sind zwei Ansätze zu unterscheiden: Zum einen können Einkommensarten, die bisher nicht der Rentenversicherungspflicht unterliegen, in die Bemessungsgrundlage einbezogen werden. Zum anderen kann die Bemessungsgrundlage, bis zu der Einkommen aus schon bisher grundsätzlich rentenversicherungspflichtigen Einkommensarten belastet wird, erhöht werden.

Bei einer Verbreiterung der Bemessungsgrundlage werden die marginalen Anreizwirkungen in den Bereichen, die schon vorher ,rentenversicherungspflichtig' waren, nicht verändert. Hingegen verschlechtern sich die Anreizbedingungen für weitere Teile der wirtschaftlichen Aktivitäten, so dass auch hier tendenziell die umfassend verstandene - Freizeit als nicht rentenversicherungspflichtige Aktivität attraktiver wird. Dies gilt um so mehr, als die Freizeit zur verstärkten Heimproduktion auch auf dem Markt zu beziehender Güter und Dienstleistungen sowie ,Schwarzarbeit' genutzt werden kann. Die Kosten der Rentenversicherung verbilligen nicht nur die eigene Freizeit, sondern verteuern auch den Bezug von - rentenversicherungspflichtig erbrachten - Fremdleistungen. Auch hier hängt die Intensität der Ausweichreaktion entscheidend von der erwarteten Rendite der Beitragszahlungen $\mathrm{ab}$.

Wirkungsunterschiede zwischen den beiden Strategien sind u.a. auch von den Unterschieden zwischen den der Regelung unterworfenen Individuen abhängig. Welche der beiden gerade charakterisierten Strategien für eine Welt (fast) identischer Individuen allokativ vorzugswürdig ist, kann ohne weitere Annahmen nicht angegeben werden. Als Tendenz kann festgehalten werden: Je besser die Möglichkeit einer Substitution von Einkommen durch Freizeit ist, desto attraktiver ist eine Ausweitung der Bemessungsgrundlage des Rentenversicherungsbeitrags,

${ }^{128}$ Die marginale Belastung des Einkommens durch eine Rentenversicherungsabgabe ist - wie schon mehrfach betont - primär dann bedeutsam, wenn die Versicherten fur eine Alternativanlage eine höhere Rendite erwarten. 
wird dabei doch die Möglichkeit zu einer Vermeidung durch Umschichtungen zwischen den verschiedenen Einkommensarten verringert. Einschränkend ist allerdings anzumerken, dass unterschiedliche tätigkeitsspezifische Komplementaritäts- oder Substitutionsbeziehungen zu Freizeit allokativ eine höhere resp. geringere Belastung der dort erzielten Einkommen nahelegen.

Nach diesen Erörterungen für den Fall intragenerational (fast) identischer Individuen muss eine Ausweitung der Betrachtung auf den Fall intragenerational unterschiedlicher Individuen erfolgen. In diesem Fall hat die Entscheidung für eine der beiden genannten Strategien zur Steigerung der Einnahmen der Rentenversicherung regelmäßig erhebliche distributive Implikationen. Während eine Verbreiterung der Bemessungsgrundlage nur diejenigen zu einer erhöhten Beitragszahlung zwingt, deren Einkommen von der bisherigen Bemessungsgrundlage nicht vollständig abgedeckt ist, sind von einer Erhöhung des Beitragssatzes alle Beitragszahler betroffen. Wenn - in Anlehnung an die deutsche Realität - unterstellt wird, dass die Rentenversicherungsbeiträge bisher vor allem auf Einkommen aus abhängiger Beschäftigung erhoben werden, so bedeutet eine Erweiterung der Bemessungsgrundlage (z.B. auch auf andere Einkommensarten, oder aber eine Erhöhung der Beitragsbemessungsgrenze, bis zu der hin Einkommen aus abhängiger Beschäftigung rentenversicherungspflichtig ist) tendenziell eine Steigerung der Beitragsbelastung vergleichsweise gut verdienender Individuen (und derjenigen, die wesentliche Teile ihres Einkommens aus anderen Quellen als abhängiger Beschäftigung erzielen).

Zur Beurteilung der wirtschaftlichen Implikationen der einen oder anderen Strategie ist auch an dieser Stelle wiederum zu berücksichtigen, inwieweit die (marginale) Beitragszahlung aus Sicht der (potenziellen) Beitragszahler den Charakter einer Ersparnisbildung oder den einer Steuer hat. Stehen erhöhten Zahlungen in der Gegenwart später auch erhöhte Rentenansprüche gegenüber, so fallen die beiden Strategien in ihren Wirkungen geringer auseinander, als wenn die erhöhte Zahlungen keine Verbesserung der ,Forderungsposition' der heutigen Aktiven in ihrer Rentenphase bedeuten. Doch selbst dann, wenn erhöhte Beitragszahlungen zu einer verbesserten Rentenposition führen, dabei aber nicht die Rendite alternativer Anlageformen erreichen, bleiben - über Liquiditätswirkungen auf Seiten der Versicherten in ihrer Aktivzeit hinaus - unterschiedliche Belastungswirkungen der beiden Strategien für Leute unterschiedlicher Einkommensposition.

Die bisherigen Ausführungen mögen den Eindruck erwecken, eine Ausweitung der Bemessungsgrundlage stelle immer eine ,Benachteiligung' besserverdienender Zwangsversicherter dar. Es ist allerdings zu beachten, dass immer dann, wenn die Rentenversicherung - auch - das Ziel einer Absicherung des Lebensstandards für die Altersphase (relativ zu dem durch Arbeitseinkommen in der Aktivphase 
erreichten Standard) hat, eine Anpassung der Beitragsbemessungsgrenze an die Entwicklung des Einkommens (aus abhängiger Beschäftigung) notwendig ist. Nur durch die Ausweitung mit der Steigerungsrate des Lohnes eines durchschnittlichen abhängig Beschäftigten ist c.p. eine entsprechende Steigerung der Beitragseinnahmen und eine entsprechende Erhöhung der Renten dauerhaft möglich. ${ }^{129}$ Die vorhergehenden Ausführungen legen also nahe, zwischen einer Erhöhung der Beitragsbemessungsgrenze entsprechend der durchschnittlichen Steigerungsrate der Arbeitsentgelte abhängig Beschäftigter, die einer Sicherung der relativen Einkommensposition der Rentner dient, und einer darüber hinausgehenden (bzw. darunter verbleibenden) Steigerungsrate zu unterscheiden. Dies ist besonders deutlich für den Fall eines intertemporal konstanten Beitragssatzes zur GRV: Hier dienen der durchschnittlichen Lohnsteigerungsrate entsprechende Erhöhungen der Beitragsbemessungsgrenze einer Partizipation der Rentnergeneration in toto an den entsprechenden Änderungen des Lohnniveaus der Beschäftigten, während darüber hinausgehende Steigerungsraten zu einer stärkeren Partizipation der Rentnergeneration führen. ${ }^{130}$

Ändert sich der Anteil des Einkommens aus rentenversicherungspflichtigen Einkommensarten am Gesamteinkommen der Aktiven, so hat dies Konsequenzen für die RV: An der Änderungsrate des durchschnittlichen rentenversicherungspflichtigen Einkommens orientierte Ausweitungen der Beitragsbemessungsgrundlage haben eine Änderung des Absicherungsniveaus eines repräsentativen Rentners verglichen mit dem Gesamteinkommen eines durchschnittlichen Aktiven zur Folge. Entsprechend gilt für die Beitragszahler, dass Umschichtungen des Einkommens hin zu nicht der Rentenversicherungspflicht unterliegenden Einkom-

129 Wobei zu beachten ist, dass die gerade getroffenen Aussagen eher fur einen ,Steady-state-Pfad' denn für reale Situationen gelten. In letzterer können die Produktivitätssteigerung (zuzüglich der Preissteigerungsrate der produzierten Produkte) übersteigende Lohnsteigerungen zur Verringerung der Beschäftigung fuhhren, und somit zu einer Schmälerung der volkswirtschaftlichen Bemessungsgrundlage der Rentenversicherungsabgabe. In diesem Falle wäre zu uberprüfen, inwieweit Senkungen des Altersabsicherungsniveaus wegen der damit möglicherweise verbundenen Verringerung der Lohnkosten des Arbeitgebers zu einer Ausweitung der Beschäftigung fuhren können, was wiederum die Beitragseinnahmen steigern und das Rentenniveau der gegenwärtigen Rentner besser sichern helfen kann als eine Erhöhung der Beitragsbemessungsgrenze.

${ }^{130}$ Die Betonung ,in toto' soll verdeutlichen, dass infolge eines sich im Zeitablauf ändernden Zahlenverhältnisses zwischen Aktiv- und Rentnergeneration eine sich interperiodisch ergebende Verbesserung der Einkommensposition der Rentnergeneration nicht bedeutet, dass sich die Einkommenssituation eines repräsentativen Rentners realiter verbessert. Sie mag sich auch nur - bei steigendem Verhältnis von Rentnern zu Aktiven - weniger verschlechtern, als sie es ohne die Steigerung der Beitragseinnahmen infolge der überproportionalen Steigerung der Beitragsbemessungsgrenze getan hätte. 
mensarten zu einer Senkung des Absicherungsniveaus (bezogen auf das Gesamteinkommen) für das Alter führen.

\subsection{Exkurs: Ausweitung des Kreises der Versicherungspflichtigen als Lösung von Finanzproblemen der GRV?}

Angesichts der häufigen Forderungen nach einer Einbeziehung aller Bezieher von (Arbeits-)Einkommen, insbesondere auch Beamter und Selbstständiger, in die Rentenversicherungspflicht, sei an dieser Stelle noch auf diesen Ansatz eingegangen. Von einer solchen Ausweitung wird erwartet, dass sich damit Beitragserhöhungen vermeiden und die Rentenversicherung sichern (stabilisieren) lassen. Dem kann nicht widersprochen werden, solange es um eine kurzfristige Stabilisierung insbesondere um die Überbrückung akuter finanzieller Engpässe - geht. ${ }^{131}$ Es gibt durch die Ausweitung des Kreises der Rentenversicherungspflichtigen bzw. der Bemessungsgrundlage unzweifelhaft einen Zugewinn an Beitragseinnahmen, so dass die Bedienung der gegenwärtigen Rentenansprüche der Alten erleichtert wird. Allerdings ist $\mathrm{zu}$ beachten, dass den kurzfristigen Einnahmesteigerungen längerfristig erhöhte Rentenansprüche gegenüberstehen. In dem Augenblick, in dem die neu Einbezogenen über alle Altersgruppen entsprechend ihres Anteils an der Gesamtpopulation der Rentenversicherungspflichtigen verteilt sind, ist der Einbeziehungsvorteil ,aufgebraucht' ${ }^{132}$ Es ist ein bekannter Vorteil einer umlagefinanzierten Rentenversicherung, dass ihre Einführung einen einmaligen Gewinn

${ }^{131}$ Wobei sich in diesem Fall die Frage stellt, ob eine Überbrückung dieses Liquiditätsengpasses mittels der Aufnahme von Krediten nicht der bessere Weg wäre. Immerhin kăme es dabei nicht zu den Problemen eines Übergangs von Beamtenpensionsansprüchen zu Rentenanwartschaften (u.U. auch verfassungsmäßig ein problematischer Systemwechsel) und der freiheitseinschränkenden Zwangsversicherung weiterer Teile der Bevölkerung. Zu beachten ist hierbei, dass selbst die Befurworter einer Ausweitung des Kreises der Versicherungspflichtigen nicht nennenswert das Argument nutzen, die bisher noch nicht Versicherten (die nicht gleichzeitig , Geringverdienende' sind) seien im Alter regelmäßig finanziell so schlecht gestellt, dass ihre vermeintliche Altersarmut ein dringendes gesellschaftliches Problem darstelle. Bisher sind Beamte und Selbststăndige im Alter eher überdurchschnittlich abgesichert, so dass eine meritorische Begründung einer Einbeziehung dieser Bevölkerungsgruppen in die Rentenversicherungspflicht nur schwerlich überzeugen kann. Auf das (umstrittene) Argument, eine Umstellung der Altersabsicherung der Beamten hin zu einer Partizipation in der GRV könne den Staat finanziell entlasten, soll hier nicht weiter eingegangen werden - es ist nicht ,rentensystematisch', und es stellt sich die Frage, ob eine Absenkung des Alterssicherungsniveaus der Beamten (relativ zu Angestellten vergleichbarer Qualifikation) nicht mit einer erhöhten Besoldung während der Aktivphase einherginge, was - zusammen mit der sofortigen liquiditätsmäßigen Belastung der öffentlichen Haushalte durch die fälligen Beitragszahlungen zur GRV - die Einsparungen gegenubber einer Beibehaltung der gegenwärtigen Regelung senken würde.

132 Vgl. DINKEL (1984a), S. 104 - dort allerdings mit Bezug auf eine höhere Erwerbspartizipation der Frauen -, WERDING (1998), S. 318f. 
in Form sofortiger Möglichkeit zur Ausschüttung von Renten infolge der Einzahlungen der Aktiven ermöglicht - zu bedenken ist aber, dass in einer Rentenversicherung, in der die Beitragszahlungen von heute die Rentenansprüche von morgen induzieren, dieser ,Einführungsgewinn' in Form einer ,Schuld' im System verbleibt. ${ }^{133}$ Durch andauernde Perpetuierung kann diese Schuld zwar intertemporal (und somit intergenerational), weitergeschoben' werden, doch die absehbaren Probleme der umlagefinanzierten Rentenversicherung in Deutschland sind gerade Reflex der Tatsache, dass die weitere Perpetuierung zunehmend schwierig wird. ${ }^{134}$ Insoweit ist nicht $\mathrm{zu}$ sehen, wie eine Einbeziehung weiterer Teile der heimischen Bevölkerung in die Rentenversicherungspflicht die strukturellen Probleme der GRV beseitigen helfen kann. ${ }^{135}$ In der Einführungsphase würde sich die Einbeziehung aber extrem vorteilhaft auf die Finanzausstattung der Rentenversicherung auswirken: Entweder die älteren bisher nicht Versicherten müssen nachversichert

${ }^{133} \mathrm{Vgl}$. WiSSENSCHAFTLICHER BEIRAT BEIM BUNDESMINISTERIUM FÜR WIRTSCHAFT (1998), S. $20-25$.

${ }^{134}$ Vielfach wird - gerade von den Befürwortern einer umlagefinanzierten Rentenversicherung auf die Möglichkeit einer Stabilisierung der Beitragssätze durch die Förderung der Migration ausländischer Arbeitnehmer nach Deutschland (und somit in die ,Zugriffssphäre' der deutschen GRV) verwiesen. Aber auch hier sei vor übertriebenen Hoffnungen gewarnt: Zum einen rechnen die Bevölkerungs- und Beitragsprognosen für die Zukunft schon mit einer gewissen Migration, d.h. nur die darüber hinausgehende Zuwanderung kann zu einer Entlastung des einzelnen Beitragszahlers gegenüber den prognostizierten Werten führen. Zum anderen ist zu bedenken, dass Migration immer mit Kosten verbunden ist - sei es auf Seiten des Auswanderungslandes, das u.U. qualifizierte Arbeitskräfte verliert, die es zur eigenen wirtschaftlichen Entwicklung benötigen würde (,brain drain'), sei es auf Seiten des Aufnahmelandes, das für die Aufnahme von Ausländern eine entsprechende Infrastruktur benötigt (Andererseits werden Ausbildungskosten gespart.). Somit ist selbst bei kurzfristig positiven Wirkungen der Zuwanderung für die Rentenversicherung nicht auszuschließen, dass die gesamtwirtschaftliche Bilanz der Migration negativ ist. Außerdem ist zu bedenken, dass einerseits die leichteste Assimilation denjenigen gelingt, deren kulturelle Differenzen zu Deutschland verhältnismäßig gering sind die solchermaßen gekennzeichneten Länder zeichnen sich aber allesamt durch eine ebenfalls vergleichsweise geringe Kinderzahl repräsentativer Mitglieder aus (und zumal in der Gruppe der Migranten) (KANE (1986, S. 99) stellt dazu fest: „Die Türken scheinen die am wenigsten assimilierte Zuwanderungsbevőlkerung zu sein und haben die höchste Geburtenhäufigkeit unter den fünf größten, in der Bundesrepublik Deutschland lebenden Ausländergruppen."), und außerdem bleiben die Anreizwirkungen des Rentenversicherungssystems hin zu einer vergleichsweise niedrigen Kinderzahl bestehen. Somit sind die Stabilisationswirkungen eines einmaligen Migrationsschubs furr das Rentenversicherungssystem eher kurzfristiger Natur. Alternativ wäre an eine dauerhafte Einwanderungspolitik zu denken, die theoretisch eine permanente Stabilisation der Rentenversicherung zur Folge haben kann. Aber auch hier bleiben Kosten der Migration bestehen, und ob die Attraktivităt eines Zuzugs nach Deutschland ohne weitere - kostspielige - Anreize gerade für leistungsfähige und -willige Ausländer attraktiv bleibt, darf angezweifelt werden.

${ }^{135}$ Für ein vergleichbares Urteil vgl. WERDING (1998), S. $318 \mathrm{f}$. 
werden, was kurzfristig erhebliche Beitragseinnahmen für die GRV bedeutet, oder aber es müssen nur Junge (Beschäftigte am Anfang ihrer Aktivzeit) eintreten, was wiederum für die nähere Zukunft nennenswerte Beitragseinnahmen ohne entsprechende Rentenzahlungen, und somit eine erhebliche Entlastung der GRV, bedeutet. Doch genau hierbei entsteht die ,Einführungsschuld' als Pendant des ,Einführungsgewinns'.

Die gerade vorgestellten Erörterungen lassen nicht erwarten, dass die Einbeziehung weiterer heimischer Bevölkerungsschichten in die GRV eine dauerhafte Entlastung der Rentenversicherung bedeuten kann. Wer der Ansicht ist, die in Deutschland praktizierte Form eines umlagefinanzierten Rentenversicherungssystems sei in ihren Fundamenten solide, für den kann es keine in der GRV begründete Indizien für die Vorteilhaftigkeit einer Ausweitung des Kreises der Versicherten geben. ${ }^{136}$ Damit ist aber schon angedeutet, wann eine solche Ausweitung möglicherweise doch angebracht sein könnte: Wenn Einzahlungen in das ,System GRV' für den repräsentativen Versicherten in Zukunft keine ,attraktive Anlage' sind, da ihre Rendite unter der anderer Anlagemöglichkeiten liegt. Dies ist aber dann der Fall, wenn die ,Anfangsschuld' des umlagefinanzierten Rentenversicherungssystems infolge einer abnehmenden Zahl von ,Trägern' immer schwerer zu perpetuieren ist. In diesem Fall stellt sich die Frage, wer die ,Alterslast' mitzutragen hat. Die Erweiterung des Kreises der (Zwangs-)Versicherten bedeutet dann die Möglichkeit der Verteilung der Kosten auf eine größere Zahl von Trägern. Wenn eine Ausweitung des Versichertenkreises geboten ist, so ist dies also tendenziell kein Zeichen für eine besondere individuelle Vorteilhaftigkeit der Mitgliedschaft - immerhin könnten viele der von einer Ausweitung Betroffenen schon bisher freiwillig beitreten, sehen eine solche Entscheidung als nicht attraktiv an. Aber mangelnde Rentabilität einer Anlage kann per se auch noch kein Grund sein, Leuten ihren Erwerb vorzuschreiben. Es ließe sich bestenfalls argumentieren, die Versichertengemeinschaft sei gerade keine freiwillige Gemeinschaft - die Nachteile des Versichertseins dürften dementsprechend gewisse Ausmaße nicht überschreiten. Da eine Überanspruchung der Mitglieder drohe, sei ein Rückgriff auf die finanziellen Mittel Außenstehender - die sich bisher nicht an den Lasten beteiligen mussten - angemessen.

Bei versicherungsfremden Leistungen ist in der Tat eine ,breite Tragung' angemessen. Es ist aber fraglich, ob diese durch eine Vergrößerung des Kreises der

${ }^{136}$ Eine Abschaffung von vermuteten, Versorgungsprivilegien' von Beamten mittels einer Einbeziehung von Beamten etc. in die GRV ist deutlich von, systemischen' Gründen zugunsten einer Einbeziehung zu trennen. Grundsätzlich lassen sich eventuelle Privilegien nämlich auch innerhalb des bestehenden Systems der Alterssicherung von Beamten (und ihrer Besoldung während der Aktivzeit) kürzen bzw. streichen. 
Zwangsversicherten gesichert werden soll. Immerhin gibt es auch die Möglichkeit einer Aufbringung der nötigen Finanzmittel aus dem Steueraufkommen. Damit entfiele die Notwendigkeit, weitere Mitglieder der Gesellschaft zwangsweise für ihr Alter zu ,versichern', um unter diesem ,Deckmantel' ihre Beiträge zur Finanzierung gesellschaftlicher Sozialleistungen einzutreiben. Ferner hätte eine solche Lösung den Vorteil, dass die Mittel für Sozialleistungen über das ,leistungsfähigkeitsorientierte' Steuersystem aufgebracht würden, und nicht über (in der bestehenden Ausgestaltung weitgehend), arbeitseinkommensproportionale' Sozialversicherungsbeiträge.

Nach Ansicht des Verfassers liefern die gerade diskutierten Argumente keinen systemimmanenten Grund für eine Ausweitung des Kreises der in der GRV Zwangsversicherten. Die Tragung allgemeiner Sozialleistungen kann (und sollte) aus dem ,Haushalt der GRV' in die ,Haushalte der Gebietskörperschaften' verlagert werden, so dass diese Lasten keine systemische Begründung für die Ausweitung der Versicherungspflicht liefern. Die Diskussion, inwieweit eine erweiterte Versicherungspflicht in der Lage ist, (vermeintliches) ,Sozialdumping' auf dem Arbeitsmarkt zu vermeiden (,Scheinselbstständigkeit', ,prekäre Beschäftigungsverhältnisse' etc.), kann nicht im Rahmen der vorliegenden Arbeit geführt werden, ebensowenig die Diskussion, inwieweit bisher nicht Pflichtversicherte im Durchschnitt für ihr Alter unzureichende finanzielle Vorsorge treffen, so dass hier ,meritorischer Eingriffsbedarf besteht. Hier mag es allerdings Gründe geben, das gerade gegebene negative Urteil über eine Ausweitung des Kreises der Rentenversicherungspflichtigen abzuschwächen.

\subsubsection{Version 3 eines Umlageverfahrens in differenzierter Sicht}

Nach den vorangegangenen - grundsätzlichen - Anmerkungen soll im Folgenden Version 3 eines Umlageverfahrens näher dargestellt werden. Ferner erfolgt eine Thematisierung der Bedeutung von Kindern innerhalb diesen Verfahrens, wobei besonders auf die sich gegenüber einer Ausgestaltung des Umlageverfahrens nach Version 1 oder 2 ergebenden Unterschiede eingegangen wird.

Für die Analyse werden die grundlegenden Annahmen aus der Untersuchung der Versionen 1 und 2 übernommen. Statt eines interpersonal konstanten Beitrags hat nun jeder Aktive jedoch auf sein Einkommen eine ,Rentenversicherungsabgabe' zu entrichten. Der Beitragssatz ist intertemporal konstant und beträgt $\{\beta \cdot 100\}$ v.H. des Einkommens. Die rentenversicherungspflichtigen Einkommen der Aktiven sind unterschiedlich hoch. 


\subsubsection{Darstellung einer umlagefinanzierten Rentenversicherung nach Version 3}

In jeder Periode muss ein Aktiver eine Beitragszahlung nach Maßgabe seines in dieser Periode bezogenen Einkommens abführen. Diese Beitragseinnahmen werden dann umgehend an die Rentner ausgezahlt. Es gilt also für jede Periode die Beziehung:

$$
\beta \cdot \sum_{i=1}^{N_{t}} y_{t}^{r v, i}=\sum_{j=1}^{N_{t-1}} r_{t}^{j}
$$

\section{Gleichung 3-12}

(mit $\beta$ : Beitragssatz; $y_{t}^{r, i}$ : rentenversicherungspflichtiges Einkommen der Person $i$ in Periode $t ; r_{t}^{j}$ : Rentenzahlung an Rentner $j$ in Periode $t ; N_{t} / N_{t-1}$ : Zahl der Beitragszahler / Rentner in Periode $t$ ).

Auch die hier vorgestellte ,Grundgleichung eines Beitragssatzverfahrens einer umlagefinanzierten Rentenversicherung' ist als Stromgrößengleichung mechanistisch, d.h. sie berücksichtigt Wechselwirkungen zwischen den Variablen nicht. Sie liefert ferner keine Aussage über die Aufteilung des Beitragsaufkommens einer Periode auf die Rentner dieser Periode. Auch über die individuellen Anreize zur Abführung von Beiträgen liefert sie keine Aussage.

\subsubsection{Das Kindeseinkommen}

In einem ,Beitragssatzverfahren' unterscheiden sich die Beitragsleistungen verschiedener Individuen zugunsten der GRV - mit steigendem Einkommen nehmen die Zahlungen an die GRV (und normalerweise auch die späteren Rentenzahlungen) zu. Doch nicht nur die Beiträge der Aktiven steigen mit ihrem Einkommen an, sondern auch die ihrer Kinder mit deren späterem Einkommen. Mithin reicht für eine Kinderberücksichtigung in der GRV eine ausschließliche Betrachtung der Zahl der Kinder eines Versicherten nicht aus, denn damit würde Ungleiches gleich bewertet - mit daraus resultierenden allokativen (und distributiven) Verzerrungen.

Bei gegebenem Beitragssatz bedeutet eine Änderung des Einkommens eine entsprechende (proportionale) Änderung der von der betroffenen Person an die RV abzuführenden Beiträge. Mithin ändert sich auch die Summe des zur Umlage an die jeweilige Rentnergeneration zur Verfügung stehenden Beitragsaufkommens. ${ }^{137}$

${ }^{137}$ Realiter gibt es Rückwirkungen auf die Arbeitsentgelte anderer Beschăftigter (und deren Beschäftigungswahrscheinlichkeit resp. Risiko von Arbeitslosigkeit). Die im Durchschnitt auftretenden Effekte dürften eher zu einer Senkung der Lohneinkommenssumme der anderen Mitglieder der Generation fuhren. 
Die Rentnergeneration in toto profitiert von einer steigenden Summe des Einkommens der nachfolgenden Generation in Form höherer Renten. Dieser Vorteil ist unabhängig von der Aufteilung der (zusätzlichen) Beitragssumme auf die Rentner.

\subsubsection{Zur Bedeutung und Berücksichtigung von Kindern bei einer umlagefinanzierten Rentenversicherung nach Version 3}

Nachdem verschiedene einzelne Grundsatzfragen einer im UV mit proportional einkommensabhängigen Beitragszahlungen ausgestalteten GRV diskutiert worden sind, soll im Folgenden auf die Bedeutung und Berücksichtigung von Kindern im Rahmen einer Ausgestaltung mit intertemporal konstantem Beitragssatz $\left(\forall t: \beta_{t} \equiv \beta\right)$ eingegangen werden.

\subsection{Externe Effekte von Kindern}

Damit eine Kinderberücksichtigung im Rahmen der RV allokativ geboten ist, müssen externe Effekte des Kinderhabens im System der RV vorliegen. Zur Verdeutlichung dieser externen Effekte wird zuerst auf die Beitragsaufkommensgleichung

$$
B_{t}\left(\beta, \sum_{i} y_{t}^{r v, i}\right) \equiv \beta \cdot \sum_{i} y_{t}^{r, i}
$$

\section{Gleichung 3-13}

(mit $B_{t}$ : Beitragsaufkommen in Periode $t$ ) verwiesen. Aus dieser lässt sich die Änderung des Beitragsaufkommens durch einen zusätzlichen Beitragszahler errechnen als

$$
\Delta_{n \rightarrow n+1} B_{t}=\beta \cdot y_{t}^{n, n+1}+\beta \cdot \sum_{i=1}^{n} \Delta_{n \rightarrow n+1} y_{t}^{r, i} \cdot{ }^{138}
$$

\section{Gleichung 3-14}

Die Beitragssumme ändert sich einerseits um die auf das rentenversicherungspflichtige Einkommen der , hinzutretenden' Person (mit Ordnungszahl $n+1$ ) entfallenden Rentenbeiträge, anderseits um die durch das Hinzukommen induzierten Änderungen in den Beitragszahlungen der anderen Personen. Für das weitere wird

${ }^{138}$ Die Gleichung bezieht sich auf einen, Gleichgewichtszustand' nach Hinzutreten eines weiteren Versicherten. Die jeweiligen Höhen des rentenversicherungspflichtigen Einkommens (und deren Änderung) inkludieren mithin alle ,Sekundäreffekte' infolge von Komplementarität bzw. Substitutivităt unterschiedlicher Arten von Beschäftigung (bzw. ihren Niederschlag in der jeweiligen Einkommenshöhe). 
unterstellt, die sich so ergebende Summe sei in toto der rentenversicherungsmäßige externe Effekt eines weiteren Aktiven. ${ }^{139}$

Grundsätzlich stellt der Gesamtbeitragsaufkommenseffekt einer Person den ,Wert' dieser Person für das Rentenversicherungssystem dar. Während der erste Teil - also das Beitragsaufkommen aus dem rentenversicherungspflichtigen Einkommen dieser Person - problemlos zugänglich ist, ist der Gesamteffekt in der Realität nicht genau zu ermitteln. Die durch das Hinzukommen einer weiteren Person bedingten Änderungen des rentenversicherungspflichtigen Einkommens anderer Versicherter sind infinitesimal, die Vermittlung erfolgt über Lohnsatzänderungen, Änderungen in der Beschäftigtenzahl, der Beschäftigungswahrscheinlichkeit etc. Die relevanten Einkommensänderungen sind diejenigen gegenüber der Situation ohne Hinzukommen des zusätzlichen Aktiven, beobachtbar aber kausal kaum in befriedigender Weise zurechenbar - sind nur die faktischen Änderungen, die zu einem erheblichen Teil auch Folge weiterer Änderungen in den Umgebungsbedingungen (von Nachfrageverschiebungen für einzelne Produkte / Betriebe / Branchen über technischen Fortschritt bis hin zu staatlichen Eingriffen etc.) sind.

\subsection{Annahme}

Für die vorliegende Arbeit wird unterstellt, dass der Gesamteffekt eines zusätzlichen Beitragszahlers auf das Beitragsaufkommen stets echt positiv ist $\left(\forall n \in N^{0}: \Delta_{n \rightarrow n+1} B_{t}>0\right) .{ }^{140}$ Der ,Wert' eines Kindes für die umlagefinanzierte Rentenversicherung wird dem (Bar-)Wert seiner Lebensbeitragszahlungen gleichgesetzt.

139 Auf eine exakte Abgrenzung der Höhe des externen Effekts wird verzichtet: Die über eine Erhöhung (resp. Senkung) der Summe der Beitragseinnahmen infolge eines zusätzlichen Aktiven vermittelten Rückwirkungen auf die Rentenhöhe seiner Eltern wird mithin gleich 0 gesetzt (Was für den Fall, dass der Rentenbeitrag des Kindes verglichen mit der Summe der Beitragszahlungen aller Mitglieder der Aktivgeneration gering ist bzw. der Anteil der Rentenzahlungen an seine Eltern im Vergleich zur Summe aller Rentenzahlungen niedrig ist - also unter ,realitătsnahen' Bedingungen eines Großsystems -, eine unproblematische Annahme darstellt.).

140 Für eine Ablehnung dieser Annahme vgl. HOMBURG und GRÄFF (1988, S. 25) mit einem Verweis auf die ,Cobb-Douglas-Produktionsfunktion'. 


\subsection{Substitution von eigenen Beitragszahlungen durch Beitragszahlungen der Kinder}

Wenn, wie gerade dargestellt, beitragszahlende Kinder für die Generation ihrer Eltern in toto positive (beitragsmäßige) externe Effekte induzieren, so entspricht es der bereits bei der Diskussion der ,Fixbeitragsversion des Umlageverfahrens' (Version 1) begründeten Logik, Beitragsleistungen der Kinder bei ihren Eltern (c.p.) rentensteigernd zu berücksichtigen.

\subsubsection{4. ,Einkommen der Kinder' statt ,Kinderzahl' als Indikator , elterlicher Leistungen' - Vergleich mit Version 1}

Im Fixbeitragsverfahren ist ausschließlich der zahlenmäßige Umfang der nachfolgenden Generation (bzw. die Zahl der Mitglieder dieser Generation, die - mehr oder minder freiwillig - dem ,Generationenvertrag' beitreten) für die Höhe der kumulierten Rentenzahlungen an die vorangegangene Generation bedeutsam. Jede Änderung dieser Zahl schlägt sich (direkt) proportional in der zur Umlage zur Verfügung stehenden Rentensumme nieder.

Beim Beitragssatzverfahren erfolgt hingegen eine Berücksichtigung sowohl von ,Quantität' als auch ,Qualität' der nachfolgenden Generation - beide Faktoren wirken auf die Summe der (rentenversicherungspflichtigen) Einkommen der nachfolgenden Generation in ihrer Aktivzeit (und somit auf das Distribuendum) ein.

Die Rentenwirksamkeit unterschiedlicher Einkommen der Kinder hat eine erhebliche Bedeutung für eine ,richtige' Bewertung elterlicher Erziehungsleistungen im Rahmen der RV: Da eine Änderung des Umfangs der Aktivgeneration nicht notwendigerweise zu einer proportionalen Änderung der Beitragseinnahmen führt, ist eine nach den jeweiligen Beitragszahlungen der Kinder differenzierende Kinderberücksichtigung allokativ geboten: ${ }^{141}$ Die Summe der Beitragseinnahmen, und eben nicht die Zahl der Kinder, ist die für die Sicherung der RV entscheidende Größe.

In einem Beitragssatzverfahren können dementsprechend für die RV gleich wertvolle ,kinderbezogene' Leistungen der Eltern durch unterschiedliche Kombinationen aus ,Kinderquantität' und ,Kinderqualität' erbracht werden. Da die späteren Rentenansprüche der Kinder wiederum proportional zu ihrem Beitrag sind,

${ }^{141}$ Die Argumentation setzt voraus, dass die Höhe des rentenversicherungspflichtigen Einkommens der Kinder nicht ausschließlich von Glück etc. bestimmt, sondern zumindest partiell endogen ist. 
besteht auch aus Sicht der ,Alterssicherungsfunktion' der Rentenversicherung kein Unterschied zwischen den verschiedenen Kombinationen. ${ }^{142}$

Aus dem gerade Gesagten folgt, dass es aus äquivalenzorientierter Sicht bei der Rentenberechnung für die Eltern keinen Unterschied machen soll, wieviele Kinder zusammen eine gegebene Beitragssumme aufbringen. Entscheidend ist vielmehr die Höhe dieser Beitragssumme. Bei einer ausschließlichen Orientierung an der Kinderzahl gibt es für die Eltern einen allokativen Fehlanreiz in Richtung ,Kinderquantität' anstelle von 'Kinderqualität' ${ }^{143}$

\subsection{Exkurs: Zum Begriff, Kinderqualität'}

So wie hier der Begriff ,Kinderqualität' gebraucht wird, besteht die Gefahr, ihn als einen Begriff aus dem ,Wörterbuch des Unmenschen' abzutun. Dies wäre aber ein verfehltes Verständnis. Kinderqualität sagt hier nichts aus über die humane Qualität von Menschen respektive über den Wert des menschlichen Lebens an und für sich. Im Sinne der anfänglichen Ausführungen zur Ökonomisierung geht es nur um einen Teilaspekt, der ausschließlich etwas über die funktionale Bedeutung von Menschen (und insbesondere Kindern) für ein gesellschaftliches Teilsystem aussagt. ${ }^{144}$ Im für die RV relevanten Sinne zeigt sich unterschiedliche ,Quali-

${ }^{142}$ Für die ,Hinterbliebenenversorgungs-' und ,Frühverrentungsperspektive' mag ein gewisser Unterschied bestehen, da in solchen Fällen regelmäßig eine Aufstockung des bis zum Eintritt des Versicherungsfalls akkumulierten Altersrentenanspruchs erfolgt. Somit mag die Zahl der Versicherten und mitversicherten Familienangehörigen wiederum bedeutsam sein (Eine Verteilung des Beitragsaufkommens auf eine größere Zahl Versicherter (,Quantität' statt ,Qualität') hat mithin eine leicht höhere Kostenbelastung der Rentenversicherung zur Folge. Diese kann aber durch eine längere wirtschaftliche Abhängigkeit ,höherwertiger' Kinder infolge längerer Ausbildung - und somit finanzieller Abhängigkeit von (Halb-)Waisenrenten im Falle des Todes eines Elternteils - kompensiert werden.).

${ }^{143}$ Zur Wirkung und Bedeutung von Substitutions- und Einkommenseffekt bei Entscheidungen über Kinderzahl und ,-qualität' vgl. z.B. BECKER und LEWIS (1974), WILLIS (1974). Zum allokativen Fehlanreiz hin zu einer Investition in ,Kinderquantität' bei Nichtberücksichtigung des Kindereinkommens im Rahmen der Festsetzung der Rentenhöhe seiner Eltern vgl. PETERS (1995), insbes. S. 166 sowie S. 173 - 177.

${ }^{144}$ BECKER (1960) definiert die Qualität von Kindern (indirekt) über die Ausgaben von Eltern zugunsten des jeweiligen Kindes, um sich dann hin in Richtung einer nutzenmäßigen Begründung des Begriffs zu begeben (,I will call more expensive children ,higher quality' children, just as Cadillacs are called higher quality cars than Chevrolets. To avoid any misunderstanding, let me hasten to add that ,higher quality' does not mean morally better. If more is voluntary spent on one child than on another, it is because the parents obtain additional utility from the additional expenditure and it is this additional utility which we call higher ,quality",, ebd., S. 173.) (vgl. auch BECKER et al. (1990) sowie CIGNO (1986)). Die Qualitäts-Definition BECKERs hat sich in der okonomischen Literatur weitgehend durchgesetzt. Auf eine Problema- 
tät' von Kindern (u.a.) in ihren unterschiedlichen Beiträgen zur RV, also in der unterschiedlichen Höhe ihres rentenversicherungspflichtigen Einkommens. Wenn ein Kind (c.p.) in seinem Erwerbsleben ein höheres rentenversicherungspflichtiges Einkommen hat, so ist es im hier vorgegebenen Sinne von höherer, Qualität'. Die ,Erwerbsorientierung ' einer derartigen Bewertung blendet die wesentlichen Seiten der menschlichen Qualitäten einer Person aus. Ein Leben mit geringeren Beiträgen zur RV kann mithin sowohl aus Sicht des Betroffenen, als auch seiner Eltern und der Gesamtgesellschaft ,besser' bzw. ,erstrebenswerter' als ein Leben mit höheren Beiträgen sein. Ferner mag es sogar für das Beitragsaufkommen der Rentenversicherung externe Effekte geben, die in der wie hier definierten, Qualität ${ }^{t}$ von Kindern keinen angemessenen Niederschlag finden. So können etwa mit einem Einkommensverzicht verbundene - ehrenamtliche Tätigkeiten indirekt durchaus zu einer Erhöhung des Beitragsaufkommens der Rentenversicherung beitragen, auch wenn nicht das Einkommen der ehrenamtlich Tätigen diese Beitragszahlungen auslöst.

Doch auch dann gibt es keinen Grund, alle unterschiedlichen Lebenseinstellungen, Präferenzen, gesellschaftliche Wertungen über die ,richtige' Ausgestaltung eines ,erfüllten' Lebens etc. bei der Konstruktion einer RV zu berücksichtigen. Wer sich für ein Leben entscheidet, dass sich primär nicht-monetären Werten widmet (sei es durch Eintritt in einen Ordensberuf, sei es durch ,Aussteigertum' etc.) oder Zahlungen zur GRV anderweitig vermeidet, der mag allen Respekt verdienen - zum Unterhalt der Elterngeneration durch die GRV trägt er unmittelbar nicht bei. Ebenso ist es fraglich, ob die Rentenversicherung das angemessene ,Instrument' ist, die Gesamtheit der externen Effekte der Aktivitäten eines Menschen zu erfassen und durch Rentengewährung anzuerkennen. Entsprechendes gilt auch für Kinder, die in ihrem Leben infolge körperlicher oder geistiger Gebrechen nicht in die RV einzahlen (können). Wenn die Gemeinschaft den Eltern von Kindern, die nicht in die RV eintreten, trotzdem die Kindererziehung, anrechnen' will was aus Gründen menschlicher Anerkennung der erbrachten Leistungen sinnvoll sein kann -, so stellen die dafür notwendigen finanziellen Mittel - je nach Ab-

tisierung wird dabei verzichtet, obwohl unterschiedliche Nuancierungen resp. Interpretationen möglich sind - je nachdem, ob der Nutzen der Kinder Grundlage der elterlichen Entscheidungen ist, oder aber die Eltern ,meritorisierend' entscheiden, welchen Zuständen sie welche Nutzenwerte ihrer Kinder zuordnen. Die in der vorliegenden Arbeit genutzte Abgrenzung von ,Qualität' geht von der, nutzenmaßigen' Wirkung weiterer Ausgaben für Kinder für ihre Eltern, wie sie bei BECKER zu finden ist, hin zu einer an ,messbaren Werten' orientierten Abgrenzung. Sie entspricht somit eher dem Ansatz von CIGNO (1991, S. 86), der die Qualität einer Person definiert als ,the maximum amount of goods to which such a person can gain access by his or her own best endeavours over a lifetime, given the levels of parental benefactions received". Aber auch im Ansatz CIGNOs wird, Qualităt' als Potenzial abgegrenzt, während in der vorliegenden Arbeit auf die Realisation abgezielt wird! 
grenzung - entweder ,versicherungsfremde Leistungen' dar (die als solche aus dem allgemeinen Steueraufkommen finanziert werden sollten), oder aber ,echte Versicherungsleistungen', die aus dem ,kinderbezogen aufzuteilenden Beitragsteil' abzugelten sind. ${ }^{145}$

\subsection{Probleme der Kinderberücksichtigung}

Im vorangegangenen Abschnitt wurde dargelegt, dass in einem Beitragssatzverfahren der RV aus allokativen Gründen die Rente der Eltern (c.p.) mit dem kumulierten Rentenbeitrag ihrer Kinder steigen soll. Auf die Probleme einer derartigen ,Kinderberücksichtigung' soll im weiteren eingegangen werden.

\subsection{Praktikabilitätsprobleme}

Der ,Nachteil' der Berücksichtigung des Kinderhabens als eigenständige Leistungskategorie, die rentenbegründend und rentensteigernd wirken kann, ist, dass der ,Wert' der eingebrachten Kinder für die GRV in einem Beitragssatzverfahren (auch ohne eine Problematisierung der Wahl der ,richtigen' Diskontrate für die Bestimmung des Barwerts zukünftiger Beitragsleistungen; vgl. hierzu Abschnitt 4.3.3.2.1) nicht unmittelbar angegeben werden kann. Während die eigene monetäre Beitragsleistung des Versicherten direkt $\mathrm{zu}$ beobachten ist, sind die späteren Leistungen der Kinder nicht bekannt, sondern müssen regelmäßig ,abgeschätzt' werden, da normalerweise ein erheblicher Teil dieser Zahlungen erst nach dem Tod der Eltern geleistet wird. Diese Abschätzung resp. Pauschalierung geht unvermeidlich mit Schätzfehlern einher sowie mit sich aus systematischen Fehlabschätzungen automatisch ergebenden allokativen Fehlanreizen etc. Die diesbezüglichen Nachteile sind mit den Vorteilen einer Kinder differenziert berücksich-

${ }^{145}$ Wichtig ist die Abgrenzung der finanziellen Berücksichtigung von Kindererziehung als ,versicherungsfremde Leistung' im Rahmen der Rentenversicherung: Die Erziehung derjenigen Kinder, die später in die Rentenversicherung eintreten, und damit die Renten sichern, stellt per se keine versicherungsfremde Leistung dar (was noch nichts uber den richtigen Umfang einer solchen Berucksichtigung aussagt - eine ,unangemessen hohe' Berucksichtigung ist ihrerseits selbstverständlich dann wieder als versicherungsfremde Leistung anzusehen, aber eben nicht die ,Kinderberücksichtigung' allgemein!). Aufgrund der Endogenităt des realisierten Einkommens ist eine Absicherung der Eltern gegen geringe Einkommen der Kinder - wie sie im obigen Text nicht ausgeschlossen wird - allokativ nicht unproblematisch. Und bei einer Absicherung gegen niedriges Einkommen des Kindes infolge von Behinderung etc. stellt sich die Frage, woran eine ,Mitgliedschaft' des Kindes in der GRV erkannt werden kann - eine Rentengewährung an alle Eltern von Nicht-Arbeitenden kann nicht Aufgabe einer Rentenversicherung mit beitragsabhängigen Renten sein. Aus pragmatischer Sicht erscheint eine steuerfinanzierte Absicherung hier als angemessen. 
tigenden Ausgestaltung der Rentenversicherung zu vergleichen, um zu einem generellen Urteil über die Angemessenheit eines solchen Verfahrens zu gelangen.

\subsection{Kinderberücksichtigung erst in der Rentenphase?}

Im zuerst analysierten Fixbeitragsansatz (Version 1) wurden die Vorteile einer Kinderberücksichtigung in der Aktivphase der Eltern ausführlich erörtert. Wenn im Beitragssatzverfahren trotzdem eine Kinderberücksichtigung in der Rentenbezugsphase stattfindet, so bedarf dies einer kurzen Diskussion der Gründe, die eine derartige Ausgestaltung erforderlich machen.

Da im Fixbeitragsverfahren (jenseits eines eventuell berücksichtigten ,Sterberisikos') keine Unsicherheit über den Beitrag der Kinder zur GRV besteht, können aus einer pauschalierenden Lösung auch keine Anreizeffekte hin zu einer ,falschen Investitionsstrategie' der Eltern gegenüber ihren Kindern existieren. Dies ist im Beitragssatzverfahren anders: Die Kinder sind eben - für die RV - unterschiedlich , wertvoll', und die Eltern haben Einfluss auf den ,Wert' ihrer Kinder. ${ }^{146}$ Eine pauschalierende Lösung, wie sie jede Berücksichtigung von Kindern schon in der Beitragsphase der Eltern darstellen muss, setzt im Beitragssatzverfahren folglich allokativ nicht die idealiter zu wünschenden Anreize. Es würde in diesem Fall darauf verzichtet, diejenigen Eltern besserzustellen, die einen besonders ,wertvollen' Beitrag zum Fortbestand der GRV leisten.

Offenkundiger Nachteil einer Kinderberücksichtigung erst in der Rentenphase sind die daraus resultierenden Konsequenzen einer (gegenüber einer Berücksichtigung schon in der Aktivphase) schlechteren Lebenseinkommensplanung der Eltern. Zwar können die Eltern bei ihrer Entscheidung über eine private Altersabsicherung ihr zu erwartendes Alterseinkommen (auch aus dem, Kinderteil' der RV) berücksichtigen, doch trotz ihrer vergleichsweise guten (Ex-ante-)Informationen über die einkommensrelevanten Eigenschaften ihrer Kinder sind einer solchen individuellen Anpassung enge Grenzen gesetzt: Zum einen hängt die spätere Einkommenshöhe der Kinder nicht nur von deren Eigenschaften ab, sondern auch von Umgebungsbedingungen, die für die Eltern (fast) nicht abzuschätzen sind. Somit besteht selbst bei guter Information über die Eigenschaften von Kindern er-

${ }^{146}$ Erstaunlicherweise wird auf diese Tatsache in Untersuchungen häufig nicht eingegangen. So weist LÜDEKE (1995, S. 179) in seiner Kritik am Ansatz der Kinderberucksichtigung von SCHMIDT et al. (1985) darauf hin, dass eine Abhängigkeit der Rentenhöhe von der Höhe des (rentenversicherungspflichtigen) Einkommens der Kinder wăhrend der Aktivzeit nur unter sehr restriktiven Bedingungen begrundbar erscheint - will dann aber gleich (und ausschließlich) die Kinderzahl als geeigneten Indikator der erfolgten Investitionen in das Humankapital der nachfolgenden Generation nutzen. 
hebliche Unsicherheit über die von ihnen in ihrem (Erwerbs-)Leben realisierten Einkommen, die wiederum ihren Niederschlag in einer Unsicherheit über die Höhe der kinderbezogenen Rente findet, und somit deren ,Nutzung' in Form verringerter Ersparnis schon in der Aktivphase der Eltern bei Risikoaversion unattraktiv erscheinen lässt.

Des Weiteren ist zu beachten, dass für einen großen Teil der Rentenversicherten der wesentliche Teil des Einkommenstransfers in die Altersphase über die zwangsweise Rentenversicherung vonstatten geht, was die Möglichkeit einer individuellen Transformation von vergleichsweise hoher kinderbezogener Altersrente in höheren Konsum während der Aktivzeit durch Verringerung anderweitiger Ersparnis erschwert. Ein Vorziehen des potenziellen Zukunftskonsums über Verschuldung ist aber infolge eines unvollständigen Kapitalmarktes kaum - und wenn, dann nur zu schlechten Konditionen - möglich.

Insoweit liegt hier also ein Zielkonflikt zwischen effizienzfördernder Ausgestaltung des Rentenversicherungssystems einerseits und einer das individuelle Alterseinkommen vergleichsweise gut prognostizierbar lassenden Ausgestaltung andererseits vor. Dieser Widerspruch zeigt sich im weiteren Verlauf der Arbeit noch mehrmals.

Zur Möglichkeit einer (partiellen) Berücksichtigung der Erziehungsleistungen schon während der Beitragsphase sei verwiesen auf Abschnitt 4.3.4.

\subsection{3. ,Elternrenten' auch für Schwiegerkinder?}

Ein systematisch relevantes Problem ist die Frage, inwieweit Einkommen der Kinder auch den Schwiegereltern zugute kommen sollen (also quasi die Einführung einer dem ,Ehegattensplitting' vergleichbaren Regelung). Eine solche Ausgestaltung hat verschiedene Vorteile, so die Internalisierung externer Effekte einer ,guten Heiratsfähigkeit' des eigenen Kindes. ${ }^{147}$ Eine besonders wichtige Eigenschaft ist die gleichmäßige Tragung eventueller ,Lasten' des Kinderhabens bzw. einer damit verbundenen Unterbrechung bzw. Aufgabe der Erwerbstätigkeit auf Seiten eines Elternteils durch die Eltern beider Partner. Zwar kann eine entsprechende Aufteilung des hieraus resultierenden Rentenausfalls auf alle (Schwieger)Eltern auch durch eine private ,Verhandlungslösung' erzielt werden, so dass eine Institutionalisierung im Rahmen der Rentenversicherung nicht unbedingt notwendig erscheint. Es sind allerdings die u.U. hohen Transaktionskosten, sowie die nach Beginn der Schwangerschaft vergleichsweise schlechte Exit-Option der El-

${ }^{147} \mathrm{Vgl}$. NERLOVE et al. (1984) sowie NERLOVE et al. (1985). 
tern der Frau, aber auch die psychischen Kosten der Wiedereinführung eines ,Brautpreises', der an die Eltern der Braut in Form einer ,Rentenzusage' zu zahlen ist, zu beachten. Das gerade Ausgeführte legt nahe, eine gemeinsame Tragung des ,Rentenrisikos' geringer Beitragszahlungen eines Ehepartners durch alle Elternteile, so für sinnvoll erachtet, institutionell im Rahmen der Rentenversicherung zu implementieren.

Dagegen spricht allerdings, dass ein ,Rentensplitting' auch negative Wirkungen auf ,Investitionen in Kinder' haben kann: Da die ,Rendite' mit den Schwiegereltern geteilt werden muss, lohnen sie sich finanziell weniger. ${ }^{148}$ Inwieweit ein Splitting insgesamt zu einer Verbesserung oder Verschlechterung der Anreize führt, ist ohne weitere Annahmen oder Untersuchungen nicht zu entscheiden.

\subsubsection{Version 4 eines Umlageverfahrens in differenzierter Sicht}

Als letzter der ,Idealtypen' eines Umlageverfahrens wird Version 4 genauer betrachtet. Diese Ausgestaltung basiert auf der Vorgabe eines intertemporal konstanten Verhältnisses der Einkommen eines repräsentativen Rentners und eines repräsentativen Mitglieds der Aktivgeneration als Ziel. Von den unterschiedenen Versionen ist die Version 4 die einzige, die eine ,Lebensstandardabsicherung' für die Rentenphase als eigenständiges Ziel hat. Dies wirkt sich, wie sich im weiteren zeigen wird, erheblich auf die Entwicklung der Beiträge (resp. Beitragssätze) aus, wenn sich Änderungen in demographischen Trends ergeben.

Im Folgenden werden zuerst verschiedene mögliche Indikatoren des (materiellen) ,Lebensstandards' vorgestellt. Daran schließt sich eine kurze Darstellung der demographischen Sensitivität des Ansatzes an, die zu einer Untersuchung der $\mathrm{Be}$ deutung von Kindern im Rahmen des Systems überleitet.

148 Zur ,Ökonomie des Heiratsmarktes' vgl. die grundlegende Arbeit von BECKER (1973/1974); vgl. auch BEHRMAN et al. (1994), BENHAM (1974), CIGNO (1991), FREIDEN (1974), NERLOVE et al. (1987); empirische Ergebnisse fur Deutschland auch in ZIMMERMANN (1985). Die Untersuchung von BEHRMAN et al. (1994) bestätigt ein ,assortive mating', eine Tendenz zur Heirat vergleichbar gut ausgebildeter Ehepartner, wobei aber Leute mit lăngerer Ausbildung Partner mit geringerer ,Arbeitsmarktorientierung' heiraten. Eine recht hohe positive Korrelation der Länge der Schulausbildung der beiden Ehepartner findet auch BECKER (1973/1974) - wobei die Korrelation der Lohnsătze der Partner zwar ebenfalls positiv, aber doch deutlich niedriger ist. 


\subsubsection{Mögliche Indikatoren des Lebensstandards}

Hat die Rentenversicherung das Ziel, einem repräsentativen Rentner einen ,angemessenen' Lebensstandard zu sichern, so lässt sich dieses Ziel am einfachsten in Form eines intertemporal konstanten Verhältnisses des Renteneinkommens zum durchschnittlichen Einkommen eines Mitglieds der Aktivgeneration ausdrücken. Es stellt sich an dieser Stelle die Frage nach der ,richtigen' Abgrenzung der miteinander zu vergleichenden Einkommen.

Da viele verschiedene Ausgestaltungsmerkmale (Arbeitseinkommen vs. Gesamteinkommen, Position nur der abhängig Erwerbstätigen oder aller Mitglieder der Aktivgeneration, Brutto- vs. Nettoeinkommen) miteinander zu kombinieren sind, so dass ein Vergleich aller einzelnen Ausgestaltungsmöglichkeiten miteinander zu umfangreich würde, werden im Folgenden unterschiedliche Ausgestaltungsmerkmale einzeln betrachtet, um daraus auf die , angemessene Kombination' zu schließen (wobei hier unterstellt wird - was angesichts der gewählten Ansatzpunkte unproblematisch erscheint -, dass zwischen den einzelnen Ausprägungen keine negativen Wechselwirkungen bestehen, so dass eine Kombination für sich ,guter' Einzelpunkte zu einem ,guten' Gesamtergebnis führt).

Vor der Erörterung möglicher Indikatoren eines durch die Rentenversicherung abzusichernden Lebensstandards soll allerdings auf die Beziehung zwischen der Größe, die Bemessungsgrundlage der Rentenversicherungsabgabe in der Aktivzeit ist, und dem gewählten Indikator des Lebensstandards eingegangen werden.

Für die vorliegende Arbeit wird - wie bereits angesprochen (vgl. 2.3.2) - die Beitragsäquivalenz der Versicherung als Referenzmaßstab für die Beurteilung der Ausgestaltung der Rentenversicherung genutzt, und darauf verzichtet, der Rentenversicherung die Realisation der jeweiligen Idealvorstellungen über die Einkommensverteilung etc. aufzuerlegen. Dieser Ansatz legt nahe, dass zwischen der Größe, auf die die Rentenversicherungsabgabe in der Aktivzeit erhoben wird, und dem Indikator des (zu versichernden) Lebensstandards ein enger (positiver) $\mathrm{Zu}$ sammenhang besteht. Nur ein solcher Zusammenhang stellt sicher, dass für Mitglieder einer Generation ihre Beiträge in der Aktivzeit - cum grano salis - in einer direkt proportionalen Beziehung zur Rentenhöhe im Alter stehen. Ferner erscheint es unter Anreizgesichtspunkten sinnvoll, dass die Aufteilung des (verfügbaren) Einkommens der Aktivzeit auf Konsum und Ersparnis keinen Einfluss auf die Höhe der Rente in der Altersphase haben soll. Bei einer Anrechnung des Alterseinkommens (oder -vermögens) auf die Höhe der Rente würde die individuelle Möglichkeit zum Einkommenstransfer in die Altersphase (noch weiter) beschränkt. Ferner stiege der Kontroll- und Verwaltungsaufwand der Rentenversicherung erheblich an, nähme doch der Umfang der für die Rentenhöhe im Alter 
bedeutsamen Faktoren (weiter) zu. Außerdem bedeutet eine Rentenrelevanz der individuellen Konsum-Ersparnis-Entscheidung der Aktiven, dass die (relative) Rentenhöhe im Alter nicht mehr Folge von Leistungen der Aktiven zugunsten der Rentenversicherung ist (Kausalprinzip), sondern auch von beitragsseitig nicht berücksichtigten Faktoren abhängt (Finalprinzip). Die Rentenversicherung gewönne bei einer solchen Ausgestaltung zunehmend ,Steuercharakter'.

Die bisherigen Ausführungen legen einen Verzicht auf die Nutzung des Gesamteinkommens eines repräsentativen Mitglieds der jeweiligen Generation als Indikator des durch die Rentenversicherung abzusichernden Lebensstandards nahe, und zwar trotz der Vorteile dieser Größe, die hier - der Vollständigkeit halber noch kurz angesprochen werden sollen: So ist das Gesamteinkommen eher als Indikator des Lebensstandards einer Person anzusehen als nur einzelne Komponenten des Einkommens. Mithin erfolgt eine ,gleichmäßige Absicherung' für das Alter: Leute mit übereinstimmendem - umfassend verstandenem - Lebensstandard in der Aktivzeit tragen in gleichem Maße zur Finanzierung der Rentenversicherung bei, um dann auch im Alter einen übereinstimmenden von der Rentenversicherung abgesicherten Lebensstandard zu haben. Die breite Bemessungsgrundlage der Rentenversicherungsabgabe ermöglicht c.p. einen niedrigen Beitragssatz, was unter Anreizgesichtspunkten per se ebenfalls positiv ist, wenn die Beitragszahlungen für die Aktiven zumindest partiellen Steuercharakter haben. Die breite Bemessungsgrundlage erschwert zudem eine ,Flucht' in nicht rentenversicherungspflichtige Aktivitäten, was ebenfalls eher vorteilhaft ist. Sie hat dabei allerdings den ,spiegelbildlichen' Nachteil, dass es keinen Einkommensbereich gibt, in dem nicht ein potenziell anreizschädigender Zugriff der Rentenversicherung erfolgt.

Ein anderer potenzieller Indikator des von der Rentenversicherung abzusichernden Lebensstandards ist die Höhe des Einkommens aus vorgegebenen Einkommensquellen (z.B. Einkommen aus abhängiger Beschäftigung, Arbeitseinkommen, Kapitaleinkommen etc.). Eine solche vergleichsweise enge Bemessungsgrundlage der Rentenversicherungsabgabe hat den Nachteil eines höheren Abgabesatzes auf das rentenversicherungspflichtige Einkommen sowie einer Abhängigkeit der Beitrags- und Rentenhöhe von der Aufteilung eines gegebenen Einkommens der Aktivzeit auf unterschiedliche Einkommensarten, so dass im Vergleich zum Lebensstandard in der Aktivzeit unterschiedliche Absicherungsniveaus erzielt werden. Haben die marginalen Beiträge eine niedrige erwartete Rendite, so gewinnen ,Gestaltungsaktivitäten' zur Umformung rentenversicherungspflichtigen- in ,nicht-rentenversicherungspflichtiges' Einkommen an Bedeutung. Außerdem werden bei derartiger Ausgestaltung Teile des Einkommens nicht zur Finanzierung der Aufgaben der Rentenversicherung herangezogen - zum Vorteil 
derjenigen, die einen erheblichen Teil ihres Einkommens in nicht-abgabepflichtigen Einkommensarten erzielen.

Eine andere Möglichkeit der Einschränkung des rentenversicherungspflichtigen Einkommens der Aktivzeit besteht darin, nicht generell Einkommen aus bestimmten Quellen von der Rentenversicherungspflicht freizustellen, sondern eine Höchstgrenze des rentenversicherungspflichtigen Einkommens einzuführen (,Beitragsbemessungsgrenze ${ }^{c}$ ), oberhalb derer das Einkommen nicht mehr belastet wird. Vorteil einer solchen Regelung ist es, die marginale Belastung höherer Einkommen c.p. zu verringern, was per se positive Leistungsanreize setzten kann. Das rentenversicherungspflichtige Einkommen hingegen wird - gegenüber der Ausgestaltung ohne Beitragsbemessungsgrenze - vergleichsweise stärker belastet. ${ }^{149}$ Spiegelbildlich zur Beschränkung der Beitragspflichtigkeit des Einkommens während der Aktivzeit erfolgt bei einem solchen Ansatz dann auch eine Beschränkung der (maximalen) Höhe der Altersrente. Der Lebensstandard der Aktivzeit wird dementsprechend für hohe Einkommen nicht für die Rentenphase abgesichert.

Bei der Beurteilung, welche Beitragsbemessungsgrundlage ,richtig' ist, hat die exakte Aufgabe der Rentenversicherung eine entscheidende Bedeutung: Je mehr die Leistungen der Rentenversicherung beitragsäquivalent sind, je geringer also das Ausmaß der im Rahmen der RV vonstatten gehenden Ex-ante-Umverteilung ist, desto eher lässt sich eine Beschränkung der Bemessungsgrundlage rechtfertigen. In diesem Fall haben vergleichsweise geringe Beitragszahlungen nicht zur Folge, dass Teile des Einkommens von der Finanzierung von Umverteilungsmaßnahmen freigestellt werden. Für die Abgrenzung des rentenversicherungspflichtigen Einkommens ist ferner von Bedeutung, inwieweit die Rentenversicherung auch eine meritorisierende Funktion hat und wie die Schutzbedürftigkeit der Aktiven eingeschätzt wird: Wie schon gesagt, bedeutet die Freistellung von Teilen des Einkommens von der Rentenversicherungspflicht, dass das staatlich (durch die RV) abgesicherte Alterseinkommen im Verhältnis zum Gesamteinkommen der Aktivzeit geringer wird. Je eher den Versicherten zugetraut wird, ein niedriges Alterseinkommen zu antizipieren und durch individuelle (freiwillige) Ersparnis (partiell) auszugleichen, desto geringer ist die Notwendigkeit zu einer vollständigen Unterwerfung des Einkommens der Aktivzeit unter die Rentenversicherungspflicht. Entsprechendes gilt auch für den Fall, dass die Gesellschaft individuelle Unterschätzungen der zu einer Absicherung des Lebensstandards notwendigen

${ }^{149}$ Eine durch eine Beitragsbemessungsgrenze begrenzte Rentenversicherungspflicht mag also im Rahmen eines (direkt) progressiven Einkommensteuertarifs besonders attraktiv sein, wird doch der marginale zwangsweise Zugriff auf höhere Einkommen infolge höherer Grenzsteuersätze durch einen Wegfall der Rentenversicherungspflichtigkeit des Einkommens verringert. 
Rücklagen (oder gar bewusste ,Unterabsicherung') ab einer gewissen Höhe des Alterseinkommens als ,tolerabel' ansieht, so dass keine besondere Schutzbedürftigkeit besteht. Die vorangegangene Argumentation gilt auch, wenn nennenswertes Einkommen aus bestimmten Quellen (wie z.B. selbständiger Erwerbstätigkeit) als Indikator vergleichsweise guter Möglichkeiten zur Abschätzung des eigenen Alterseinkommens (und des Einkommensbedarfs im Alter) angesehen wird, und somit eine Freistellung diesbezüglicher Einkommen von der Versicherungspflicht meritorisch ,unproblematisch' erscheint. Eine Freistellung sehr geringer Einkommen mag damit begründet werden, dass Beitragszahlungen auf diese Einkommen nicht ansatzweise in der Lage sind, in einer der Idee der Beitragsäquivalenz folgenden Ausgestaltung der Rentenversicherung eine Altersrente in einer Höhe zu induzieren, die dem Ziel der Sicherung eines gewissen absoluten Lebensstandards entspricht. ${ }^{150}$ Abschließend ist noch auf ,Erfassungsprobleme' sowie auf die Möglichkeit negativer Einkünfte hinzuweisen: beides ist eher für Gewinneinkünfte bedeutsam; die praktischen Probleme einer Beitragsbemessung können den Verzicht auf eine Heranziehung dieser Einkommensarten zur Rentenversicherungspflicht aus pragmatischen Erwägungen rechtfertigen.

Wird eine besondere Schutzbedürftigkeit abhängig Erwerbstätiger konstatiert, z.B. da diese im Alter eine vergleichsweise geringe Möglichkeit zur Erzielung nennenswerten Arbeitseinkommens besitzen, sowie einer besonderen Bedeutung des Erwerbseinkommens an der Summe der Einkommen, so mag die Nutzung der Höhe des Bruttoeinkommens aus abhängiger Beschäftigung als Bemessungsgrundlage der Rentenversicherungsabgabe attraktiv erscheinen. Dabei kann gleichzeitig eine Beitragsbemessungsgrenze eingeführt werden; diese dient dann als Indikator des Lebensstandards, oberhalb dessen die Gesellschaft eine weitere zwangsweise Altersabsicherung für nicht notwendig erachtet. ${ }^{151}$ Ein Rückgriff auf das Einkommen aus abhängiger Beschäftigung hat weitere ,Vorteile': Da das bezeichnete Einkommen nicht-negativ ist, ergeben sich keine Probleme mit der Behandlung negativer Einkommen. Ferner ist es regelmäßig mit liquiden Mitteln verbunden, so dass die Zahlung an die RV gesichert erscheint. Dies gilt um so mehr, als der Arbeitgeber zur Abführung des Beitrags des Beschäftigten (quasi in dessen Auftrag) verpflichtet werden kann. Es existiert hier also eine Bürokratie (Lohnbuchhaltung), die die Überweisung mit recht geringem (zusätzlichen) Auf-

${ }^{150}$ Zum einen ist zu argumentieren, dass der Versicherte in seiner Aktivzeit dann nicht seinen Lebensunterhalt aus dieser Einkunftsart bestritten hat, so dass keine Notwendigkeit besteht, aus einem auf diesem Einkommen basierenden Altersrentenanspruch heraus die Lebenshaltung im Alter zu bestreiten. Ferner ist zu bedenken, dass die Verwaltungskosten bei Erfassung sehr geringer Einkünfte u.U. höher sind als die Beitragsleistungen, eine Beitragserhebung also nicht sinnvoll ist.

${ }^{151}$ Zur ökonomischen Bedeutung der Höhe der Beitragsbemessungsgrenze vgl. 3.3.2.3.2.2. 
wand sicherstellen kann und die notwendigen Informationen (Höhe des Einkommens aus der Beschäftigung beim jeweiligen Arbeitgeber) besitzt.

Wurde bisher die ,Aufbringungsseite ${ }^{6}$ betrachtet, so soll im Folgenden auf die Rentenzahlungen eingegangen werden: Eine für die vorliegende Arbeit bedeutende Frage ist, inwieweit sich die Messung der relativen Einkommensposition von Rentnern an dem Verhältnis der Brutto- oder der Nettoeinkommen zu orientieren hat, wobei hier zusätzlich zur Einkommensteuerzahlung die eventuell anfallenden (Zwangs-)Beiträge zu den Systemen der Sozialen Sicherung besondere Beachtung verdienen. Für die weitere Argumentation wird unterstellt, dass die Höhe des Bruttoeinkommens eines repräsentativen Mitglieds der Aktivgeneration oberhalb der eines repräsentativen Mitglieds der Rentnergeneration liegt. Bei direkt progressiver Ausgestaltung des Einkommensteuertarifs bedeutet dies, dass sowohl der Grenz- als auch der Durchschnittssteuersatz auf das Einkommen eines repräsentativen Aktiven über den jeweiligen Werten für einen repräsentativen Rentner liegen. Daraus folgt, dass bei Festlegung des ,Absicherungsniveaus' mit Bezug auf Bruttoeinkommensgrößen die ,Nettoabsicherung' der Rentner relativ höher als der ausgewiesene Bruttowert ist. ${ }^{152}$ Für eine stationäre Umgebung ist der gerade beschriebene Sachverhalt unproblematisch - zwischen den Brutto- und den Nettoeinkommensgrößen besteht eine invariante Beziehung.

Kommt es aber intertemporal zu einer Steigerung der Bruttoeinkommen der Aktivgeneration, so bedeutet dies, wenn keine Anpassung des Steuertarifs erfolgt, bei einer Orientierung an Bruttoeinkommensgrößen regelmäßig eine Steigerung (auch) des relativen Netto-Absicherungsniveaus der Rentner. Dies gilt - ohne Steuertarifanpassung - sowohl für den Fall, dass die Einkommenssteigerung der Mitglieder der Aktivgeneration Folge von Preisniveauerhöhungen nachvollziehenden Anpassungen des Bruttolohns ist (also bei intertemporal konstantem realem Bruttoeinkommen) - hier infolge der ,kalten' Progression im Steuertarif -, als auch bei Steigerungen des Bruttorealeinkommens - hier infolge der ,gewöhnlichen' Progression. Schon bei der Betrachtung ausschließlich der Wirkung einer progressiven Einkommensbesteuerung wird deutlich, dass die Abstellung des Sicherungsniveaus der Mitglieder der Rentnergeneration auf das Bruttoeinkommen zu einer intertemporalen Verschiebung des relativen Nettoabsicherungsniveaus führt, wenn nicht der Einkommensteuertarif an ,Niveauverschiebungen' des Einkommens der Aktiven ,angepasst' wird. Eine exakte Nachvollziehung der Effekte würde den Rahmen der Arbeit sprengen, ohne weitergehende Einsichten zu vermitteln: In der bisherigen (verkürzten) Betrachtung wurde nur auf die (durch-

${ }^{152}$ Zur Verdeutlichung ein Verweis auf die gegenwärtige Situation in den Alten Bundesländern: Im Jahre 1998 betrug das nominale Rentenniveau brutto 48,5 v.H., netto jedoch 70,5 v.H. (VERBAND DEUTSCHER RENTENVERSICHERUNGSTRÄGER (1999), S. 208; vorlăufige Werte). 
schnittliche resp. marginale) Belastung der Mitglieder der Aktivgeneration abgestellt. Wenn das Renteneinkommen aber ebenfalls besteuert wird, so ergeben sich durch Steigerungen der Rentenhöhe auch hier Progressionswirkungen, so dass die oben genannten Schlussfolgerungen bezüglich der ,Verbesserung' der Nettoeinkommensposition der Rentner relativiert werden müssen, da möglicherweise der Durchschnittssteuersatz eines repräsentativen Rentners stärker steigt als der eines repräsentativen Aktiven, und sich somit die relative Nettoeinkommensposition des repräsentativen Rentners verringern kann.

Eine besondere Bedeutung der Unterscheidung zwischen einer brutto- und einer nettoorientierten Lebensstandardabsicherung ergibt sich in Deutschland aus der Tatsache heraus, dass bei Renteneinkommen nur ein geringer Teil (der ,Ertragsanteil', der bei Altersrenten typischerweise ungefähr 30 v.H. der Rentenleistung ausmacht) als steuerbares Einkommen erfasst wird. ${ }^{153}$ Durch diese ,Mindergewichtung' von Renteneinkünften kommt es bei Steigerungen des Bruttoeinkommens und ,bruttoorientierter' Rentenhöhe - in Verbindung mit dem gültigen Einkommensteuertarif - zu einer besonders starken Verbesserung der relativen Nettoeinkommensposition des repräsentativen Rentners.

Bei der gerade erfolgten Diskussion einer Belastungswirkung ist ausschließlich auf die Sinnhaftigkeit der Berücksichtigung unterschiedlicher steuerlicher Belastungen im Rahmen der Festlegung eines ,Altersabsicherungsniveaus' eingegangen worden. Insbesondere wurden weitere Zwangsabgaben von den Einkommen nicht betrachtet. Dies ist aber notwendig, um zu sinnvollen Vergleichen über den ,Lebensstandard' der jeweiligen repräsentativen Mitglieder einer Alterskohorte zu gelangen.

Die von den Mitgliedern der Aktivgeneration von ihrem Arbeitslohn abzuführenden Beiträge zur Rentenversicherung schieben einen - weiteren - Keil zwischen Brutto- und Nettoeinkommen der abhängig Beschäftigten. ${ }^{154}$ Ändert sich der Anteil des Abzugs am Arbeitseinkommen im Zeitablauf nicht, so kann die Beitrags-

${ }^{153}$ Vgl. EStG $\$ 22$ Nr. 1. Der ausgewiesene ,Ertragsanteil' sinkt mit dem Alter des erstmaligen Rentenbezugs. Für einen Renteneintritt mit dem vollendeten 60 sten Lebensjahr beträgt er 32 v.H., um mit (fast) jedem Jahr spăteren Renteneintritt um 1 v.H. zu sinken bis zu einem Wert von 2 v.H. für einen Renteneintritt ab dem 97sten Lebensjahr.

${ }^{154} \mathrm{Da}$ an dieser Stelle keine quantitative Aussage uber die Höhe des ,Keils' getroffen werden soll, ist es für die weitere Argumentation nicht notwendig, die Frage, inwieweit der ,Arbeitgeberanteil' der Beiträge zur GRV mit in die Betrachtung einbezogen werden muss oder nicht, vertiefend zu erörtern. Eine Einbeziehung des ,Arbeitgeberanteils` würde zu einer argumentativen Komplizierung fuhren, da er steuerlich in Gänze ,freigestellt' wird, was beim ,Arbeitnehmeranteil' nicht der Fall ist. 
belastung der Arbeitnehmer bei der Festlegung der Bruttoeinkommensposition der Rentner berücksichtigt werden. ${ }^{155}$ Dies erscheint insoweit angemessen, als die Beitragszahlungen (des Arbeitnehmers) zur GRV zwangsweise abgeführt werden, und dem Arbeitnehmer mithin nicht zur Lebensführung zur Verfügung stehen sie schmälern seinen Lebensstandard in der Aktivzeit. Bei einem gleich hohen Bruttoeinkommen hat der Rentner, der keine Beiträge zur GRV zahlen muss, ein höheres Nettoeinkommen. ${ }^{156}$ Bei intertemporaler Konstanz der Beiträge zu den Systemen der Sozialen Sicherung folgt aus ihrer primären Belastung der Aktiven noch keine Empfehlung zu einer Brutto- oder Nettoorientierung des Absicherungsniveaus der GRV. Dies ändert sich jedoch, wird zu einer demographische Verschiebungen berücksichtigenden Betrachtung übergegangen: Wie im weiteren Verlauf der Ausführungen deutlich wird (vgl. 3.3.3.2), führt bei einer ,Lebensstandardabsicherung' eine Abnahme der Wachstumsrate der Stärke der Aktivgeneration (c.p.) zu höheren Beitragsverpflichtungen. Werden diese Belastungen bei der Festlegung des ,Referenzlebensstandards' nicht berücksichtigt, so verbessert sich die am verfügbaren Einkommen der Mitglieder der jeweiligen Generation gemessene relative Einkommensposition der Rentner bei abnehmendem Generationenumfang.

Abschließend bleibt noch auf die Berücksichtigung von Schwankungen in der Höhe des (rentenversicherungspflichtigen) Einkommens im Rahmen der ,Lebensstandardabsicherung' einzugehen. Hierbei sind zwei unterschiedliche ,Komponenten' zu unterscheiden: Zum einen Änderungen des durchschnittlichen (Arbeits-)Einkommens eines repräsentativen Mitglieds der Aktivgeneration, zum anderen individuelle Verschiebungen der relativen Einkommensposition eines Aktiven im Zeitablauf (z.B. infolge von Senioritätsentlohnung, Karriere, Änderung der Knappheit seiner Qualifikationen etc.). Während der erstgenannte Punkt durch die Festlegung des Absicherungsniveaus eines repräsentativen Mitglieds der jeweiligen Generation automatisch erfasst wird, ist eine solche Regelung für den zweiten Punkt bisher nicht erörtert worden. Hier bietet es sich an, die durchschnittliche relative Einkommensposition des jeweiligen Aktiven (repräsentiert durch das Verhältnis seines (rentenversicherungspflichtigen) Bruttoeinkommens zum durch-

155 Wobei es bei genauer Betrachtung zu weiteren Problemen kommt: Immerhin werden Teile der Rentenversicherungsbeiträge der abhängig Beschäftigten als die Bemessungsgrundlage der Einkommensbesteuerung mindernd berücksichtigt - was zu unterschiedlichen ,Entlastungen' in Abhängigkeit vom jeweiligen Grenzsteuersatz fuhrt.

${ }^{156}$ In der Argumentation ist bewusst nur auf die Beiträge zur GRV eingegangen worden. Die Beitragszahlungen zur Gesetzlichen Kranken- und Pflegeversicherung (GKV resp. GPV) sind insoweit anders zu bewerten, als zu diesen auch die Rentner von ihrem (Renten-)Einkommen Beiträge abzufuihren haben, so dass der systemische Unterschied zwischen Aktiven und Rentnern diesbezulglich entfällt. 
schnittlichen (rentenversicherungspflichtigen) Bruttoeinkommen aller im jeweiligen Jahr abhängig Erwerbstätigen) als Maßstab zu nehmen: Zum einen werden hierdurch individuelle Einkommensänderungen, die (direkt) proportional zur Änderung des durchschnittlichen Einkommens der abhängig Beschäftigten verlaufen, ausgeblendet (was auch der Fall sein soll, werden sie doch - wie bereits erwähnt im erstgenannten Punkt berücksichtigt), andererseits werden über das Erwerbsleben hinweg Schwankungen geglättet (die durchschnittliche Einkommensposition eines Versicherten während seiner Erwerbstätigkeit ist sicher repräsentativer für seinen ,durchschnittlichen Lebensstandard' als die eines einzelnen Arbeitsjahres).

Die vorangegangenen Ausführungen legen es nahe, die durchschnittliche Nettoeinkommensposition aus abhängiger (und rentenversicherungspflichtiger) Beschäftigung als Indikator des durch die GRV für das Alter abzusichernden Lebensstandards - und auch als Bemessungsgrundlage für die Beitragszahlungen zur GRV - zu nutzen. ${ }^{157}$

\subsubsection{Determinanten des Beitragssatzes zur Rentenversicherung bei einkommensbezogener Absicherung des Lebensstandards}

Für die formale Analyse des Ziels einer Sicherung der relativen Einkommensposition von Rentnern empfiehlt es sich zwischen einem Bruttoabsicherungssatz $(\phi)$ und einem Nettoabsicherungssatz $(\widetilde{\phi})$ zu unterscheiden. Der Bruttoabsicherungssatz setzt das Renteneinkommen in Beziehung zum Bruttoeinkommen des Rentners während seiner Aktivzeit, so dass für das Verhältnis von ,Bruttorenteneinkommen' und ,Bruttoarbeitseinkommen' gilt:

$\frac{\text { Bruttorenteneinkommen }}{\text { Bruttoarbeitseinkommen }}=\frac{\phi \cdot y_{t}}{y_{t}} \equiv \phi$,

Gleichung 3-15

d.h. das Verhältnis bleibt, wie es sich in der Wahl des Absicherungsziels schon als Vorgabe manifestiert hat, intertemporal konstant. ${ }^{158}$ Der Nettoabsicherungssatz

${ }^{157}$ Hierbei erscheint eine individuelle Berucksichtigung interpersonell unterschiedlicher Differenzen zwischen Brutto- und Nettoeinkommen nicht sinnvoll - zum einen aus Gründen des Verwaltungsaufwands, zum anderen, da sich die Beitragszahlung auf das nicht von steuerlichen Gestaltungen abhängige Bruttoeinkommen bezieht.

${ }^{158}$ Auf eine entsprechende formale Darstellung der Definition des Nettoabsicherungssatzes kann verzichtet werden. Es genügt in der definitorischen Beziehung ,Brutto(renten)einkommen durch ,Netto(renten)einkommen' zu ersetzen. 
hingegen setzt das Renteneinkommen in Beziehung zum Nettoeinkommen des Rentners während seiner Aktivzeit.

Es versteht sich, dass die gerade genannten Absicherungssätze nicht mit den Beitragssätzen zur Rentenversicherung übereinstimmen. Die Umstände, die die Beziehung zwischen Beitrags- und Absicherungssätzen bestimmen, sollen im Folgenden - unter restriktiven Annahmen - untersucht werden: Welche Faktoren sind für die Höhe (und Entwicklung) des Beitragssatzes eines umlagefinanzierten Rentenversicherungssystems mit am Arbeitseinkommen orientierter Lebensstandardabsicherung bedeutsam?

\subsection{Annahmen}

Wie schon in den vorangegangenen Teilen der Arbeit wird auch hier ein 2-Generationen-Ansatz betrachtet. Jede Generation wird durch einen ,repräsentativen Vertreter' dargestellt. Sein jeweiliges Einkommen ist schon das ,Nach-SteuerEinkommen'. Beiträge zur Rentenversicherung sind ausschließlich von Mitgliedern der Aktivgeneration zu zahlen. Ihre Höhe ist direkt proportional zur Höhe des Nach-Steuer-Einkommens der Versicherten.

Die obigen Annahmen stellen für die Untersuchung von Bestimmungsfaktoren des Rentenversicherungsbeitrags keine wesentlichen Einschränkungen dar, erleichtern aber eine - verständliche - Darstellung der grundlegenden Sachverhalte. Die Gründe für eine Einschränkung der Betrachtung ausschließlich auf rentenversicherungspflichtige Einkommen in der Aktivphase und auf Renteneinkommen in der Altersphase sind bereits zuvor thematisiert worden. Der Verzicht auf eine Modellierung der Einkommensbesteuerung ermöglicht es, die unterschiedliche ,Gewichtung' einzelner Einkommensarten (so die bereits erwähnte ,Untergewichtung' von Renteneinkommen) zu vermeiden, ebenso die Rückwirkungen einer Verminderung der steuerlichen Bemessungsgrundlage der Aktivzeit um Teile der Rentenversicherungsbeiträge und die Folgen der progressiven Ausgestaltung des Einkommensteuertarifs auszublenden.

\subsection{Analyse}

Entgegen der geforderten Orientierung an der Nettoeinkommensposition wird aus analytischen Gründen zuerst der Fall einer Nicht-Berücksichtigung der Rentenversicherungsabgaben der Mitglieder der Aktivgeneration bei der Festlegung des ,Absicherungsniveaus' betrachtet. 
Für einen gegebenen ,Bruttoabsicherungssatz' $\phi(\phi \in[0 ; 1])^{159}$ gilt definitionsgemäß:

$r_{t} \equiv \phi \cdot y_{t}$

Gleichung 3-16

(mit $y_{t}$ : Einkommen eines repräsentativen Beitragszahlers in Periode $t$ ).

Der Bruttoabsicherungssatz wird hierbei nicht von eventuellen Beitragsänderungen für die Mitglieder der Aktivgeneration - z.B. infolge veränderter demographischer Umgebungsbedingungen - beeinflusst. Und dass sich der Beitrag des repräsentativen Mitglieds der Aktivgeneration bei Änderungen der Bevölkerungszusammensetzung (c.p.) ändern muss, ergibt sich aus der Beitragsaufkommensgleichung: Da die Summe der Beitragszahlungen der Mitglieder der Aktivgeneration gerade der Summe der Rentenzahlungen an die Mitglieder der Rentnergeneration entspricht, gilt:

$\beta_{t} \cdot y_{t} \cdot N_{t} \stackrel{!}{=} \phi \cdot y_{t} \cdot N_{t-1} \Leftrightarrow \beta_{t}=\phi \cdot \frac{N_{t-1}}{N_{t}}=\phi \cdot \frac{1}{1+g_{t}} . \quad$ Gleichung 3-17

Das Verhältnis der ,Nach-Rentenversicherungsabgaben-Einkommen' beträgt somit (unter den Annahmen $y_{t}>0$ und $\beta_{t}<1$ ) 160

159 Wobei ein ökonomisch sinnvoller Bruttoabsicherungssatz größer als 0 und kleiner als 1 sein sollte - immerhin müssen die Aktiven von ihrem ,Bruttoeinkommen' noch den Rentenversicherungsbeitrag abfuhren, so dass schon bei $\phi=1$ (und erst recht bei 1 ubersteigenden Werten von $\phi$ ) das verfugbare Einkommen eines Rentners das eines Aktiven ubersteigt. Zwar ist nicht ohne weiteres davon auszugehen, dass die Mitglieder der Gesellschaft ein Lebenseinkommensprofil, bei dem das (verfügbare) Renteneinkommen das (verfügbare) Aktiveinkommen ubersteigt, ablehnen, für die weitere Betrachtung wird dieser Fall aber ausgeschlossen. Als Begründung hierfür kann eine Vorliebe der Gesellschaftsmitglieder fur eine bedarfsgerechte intertemporale Einkommensaufteilung dienen, wobei wăhrend der Aktivzeit infolge eventueller Kindererziehung ein relativ höherer Einkommensbedarf als in der Rentenphase besteht; weitere mögliche Begründungen sind Risikoaversion im Falle uber ihre Lebensdauer unsicherer Individuen sowie Kapitalmarktunvollkommenheiten, die eine individuelle Abweichung vom durch die Rentenversicherung für das Einkommen aus abhängiger Beschăftigung vorgegebenen Lebenseinkommensprofil durch Vorziehung zuküftigen Einkommens in die Gegenwart gegenüber einer Verschiebung gegenwärtigen Einkommens in die Zukunft erschweren.

Außerdem wird - wie in der gesamten Arbeit - die Existenz einer nachfolgenden Generation unterstellt, d.h. $g_{t}>-1$. 


$$
\frac{\phi \cdot y_{t}}{y_{t}-b_{t}}=\frac{\phi \cdot y_{t}}{y_{t}-\beta_{t} \cdot y_{t}}=\frac{\phi \cdot y_{t}}{y_{t}-\phi \cdot y_{t} \cdot \frac{1}{1+g_{t}}}=\frac{\phi}{1-\phi \cdot \frac{1}{1+g_{t}}}, \quad \text { Gleichung 3-18 }
$$

womit gilt:

$$
\frac{d \frac{\phi \cdot y_{t}}{y_{t}-b_{t}}}{d g_{t}}=-\phi^{2} \cdot \frac{1}{\left(1+g_{t}\right)^{2}} \cdot \frac{1}{\left(1-\phi \frac{1}{1+g_{t}}\right)^{2}}<0
$$

d.h. mit abnehmender Wachstumsrate des Umfangs der Aktivbevölkerung steigt die am verfügbaren Einkommen gemessene Einkommensposition der Rentner relativ zu der der Aktiven. Die Zahl der von jedem Aktiven zu unterhaltenden Rentner nimmt $\mathrm{zu}$ - und da deren Pro-Kopf-Renteneinkommen sich im Verhältnis zu dem Einkommen des Aktiven nicht verringert, muss letzterer einen höheren Anteil seines Einkommens an die Rentner überweisen.

Zum Vergleich sei jetzt auf das Verfahren einer ,Nettoorientierung' eingegangen, also auf ein Verfahren, bei dem das Renteneinkommen eines repräsentativen Rentners in einem konstanten Verhältnis zum Einkommen abzüglich der darauf entfallenden Rentenversicherungsbeiträge eines repräsentativen Arbeitnehmers stehen soll.

In diesem Fall gilt definitionsgemäß

$r_{t} \equiv \widetilde{\phi} \cdot\left(y_{t}-b_{t}\right)$

Gleichung 3-20

(mit $\widetilde{\phi}:$,Nettoabsicherungssatz' $(\widetilde{\phi} \in[0 ; 1]))$,

woraus für das ,Nach-Abgaben-Einkommensverhältnis' folgt (unter der Annahme $\left.\forall t: 0<b_{t}<y_{t}\right)$ :

$\frac{r_{t}}{y_{t}-b_{t}}=\widetilde{\phi}$. 
Es bleibt also - wie als Ziel vorgegeben - intertemporal konstant und wird von Änderungen der Umgebungsbedingungen (wie eben Änderungen in der Wachstumsrate der Aktivbevölkerung) nicht berührt.

Aus der Beitragsaufkommensgleichung

$$
b_{t} \cdot N_{t}=r_{t} \cdot N_{t-1}=\widetilde{\phi} \cdot\left(y_{t}-b_{t}\right) \cdot N_{t-1}
$$

folgt (wegen $N_{t} \equiv N_{t-1} \cdot\left(1+g_{t}\right)$ )

$$
b_{t}=\frac{N_{t-1} \cdot \widetilde{\phi} \cdot y_{t}}{N_{t}+N_{t-1} \cdot \widetilde{\phi}}=\frac{\widetilde{\phi} \cdot y_{t}}{1+g_{t}+\widetilde{\phi}}
$$

Hiermit lässt sich das Verhältnis der Bruttoeinkommen errechnen als

$$
\frac{r_{t}}{y_{t}}=\frac{\tilde{\phi} \cdot\left(y_{t}-b_{t}\right)}{y_{t}}=\tilde{\phi} \cdot\left(1-\frac{b_{t}}{y_{t}}\right)=\widetilde{\phi} \cdot\left(1-\frac{\tilde{\phi}}{1+g_{t}+\widetilde{\phi}}\right)
$$

woraus folgt:

$$
\frac{d \frac{r_{t}}{y_{t}}}{d g_{t}}=\tilde{\phi}^{2} \cdot \frac{1}{\left(1+g_{t}+\tilde{\phi}\right)^{2}}>0 \text {. }
$$

Mit abnehmender Wachstumsrate der Aktivbevölkerung sinkt das Bruttoeinkommen der Rentner relativ zu dem Bruttoeinkommen der Aktiven. Die Letzteren müssen von ihrem Einkommen aber immer größere Teile zur Finanzierung der Renten der Mitglieder der Ruhestandsgeneration aufwenden, d.h. (auch) ihr verfügbares Einkommen sinkt (c.p.). ${ }^{161}$

${ }_{161} \frac{d\left(y_{t}-b_{t}\right)}{d g_{t}}=\frac{\tilde{\phi} \cdot y_{t}}{\left(1+g_{t}+\widetilde{\phi}\right)^{2}}>0$ (für $\tilde{\phi}>0$ ). 


\subsection{Erweiterungen}

Für die Analyse im vorangegangenen Abschnitt wurde auf die Berücksichtigung der Einkommensbesteuerung verzichtet. Im Folgenden soll kurz auf die qualitativen Änderungen bei einer Berücksichtigung eingegangen werden.

Da die gegenwärtige Ausgestaltung der deutschen Einkommensbesteuerung tendenziell zu einer relativ höheren Belastung von Arbeitseinkommen gegenüber Renteneinkommen führt (zum einen, da mit steigendem zu versteuerndem Einkommen die durchschnittliche steuerliche Belastung zunimmt, zum anderen, da nur ein Bruchteil des Renteneinkommens zu versteuern ist), würde eine ,Bruttoabsicherung' bei abnehmendem Wachstum des Umfangs der Aktivbevölkerung den schon beschriebenen Effekt einer Verbesserung der Relativposition der Rentner bezüglich der verfügbaren Einkommen noch verstärken. Da Teile der Arbeitnehmerbeiträge zur GRV im Rahmen der Einkommensbesteuerung deren Bemessungsgrundlage verringern, bedeutet eine Berücksichtigung der Steuerwirkungen bei einer Nettoabsicherung, dass die höheren Beiträge der Aktiven infolge einer verringerten Wachstumsrate der Aktivbevölkerung die steuerliche Belastung der Aktiven tendenziell senken, d.h. ihr nach Steuern und Abgaben verfügbares Einkommen sinkt um weniger als den von ihnen mehr abzuführenden Rentenversicherungsbeitrag. Ein Teil dieses, Gewinns' kommt wiederum den Rentnern in Form einer vergleichsweise höheren Rentenzahlung zugute. ${ }^{162}$

\subsubsection{Zur Bedeutung und Berücksichtigung von Kindern bei einer umlagefinanzierten Rentenversicherung nach Version 4}

Im Folgenden soll kurz auf die Bedeutung und die Berücksichtigung von Kindern für den Fall einer Absicherung des Nettolebensstandards durch die GRV eingegangen werden. Auf eine explizite Betrachtung des ,Bruttoabsicherungsansatzes' wird verzichtet, da die schon erläuterten konzeptionellen Probleme des Verfahrens im Falle demographischer Verschiebungen eine ,unkorrigierte' Nutzung des Ansatzes im Falle einer anhaltenden Abnahme der Nettoreproduktionsrate kaum möglich erscheinen lassen. ${ }^{163}$

162 Die Wirkungskette hört aber nicht an der beschriebenen Stelle auf. Immerhin kommt es im gerade beschriebenen Verlauf (c.p.) bei steuerlicher Freistellung der Beiträge zur GRV zu einer Abnahme des Steueraufkommens. Damit stellt sich die Frage, wie dieses ,kompensiert' wird, und wie dementsprechend die Belastungswirkungen hierbei auf die Generationen verteilt sind.

${ }^{163}$ Sind die demographischen Verschiebungen hingegen gering, ist ein Verweis auf die Ausführungen zum ,Beitragssatzverfahren' (mit intertemporal fixem Beitragssatz) möglich, da der dann (fast) konstante Beitragssatz keine treibende Kraft für eine Änderung des Nettoabsicherungsniveaus ist. 
Für den Fall einer Bevölkerung mit konstanter ,Altersstruktur ${ }^{164}$ und einer proportionalen Einkommensbesteuerung entspricht der ,Lebensstandardabsicherungsansatz' der GRV einem Fixbeitragssatzverfahren. Aufgrund der Stationarität ist der Anteil der (Brutto-)Einkommen der Aktiven, der zur Sicherung eines vorgegebenen relativen Lebensstandards der Rentner benötigt wird, intertemporal konstant. Da sich der Anteil der ,Alten' im Verhältnis zu den Mitgliedern der Aktivgeneration im Zeitablauf nicht ändert, bedeutet die Sicherung einer vorgegebenen (und konstanten) relativen Einkommensposition der Rentner, dass ein konstanter Anteil der Summe der Arbeitseinkommen an die Rentner, umverteilt' werden muss - entsprechend dem Fall eines intertemporal konstanten Beitragssatzes. Für die Bedeutung von Kindern und die Möglichkeiten ihrer Berücksichtigung im Rahmen der GRV kann in diesem Fall also auf die entsprechenden Untersuchungen zum Fixbeitragssatzsystem verwiesen werden (vgl. 3.2.1.3).

Bei direkt progressiver Besteuerung der Einkommen (und verstärkt bei vergleichsweise geringer Besteuerung von Renteneinkommen) ist es möglich, den Beitragssatz im Zeitablauf tendenziell leicht zurückzunehmen, da sich die anteilige Verteilung der Steuerlasten, die bei der Festlegung der Renten- resp. Beitragshöhe berücksichtigt wird, hin zu einer stärkeren Tragung durch die Mitglieder der Aktivgeneration verschiebt. Bei nicht allzu großen Veränderungen der relativen Steuerbelastung der Mitglieder der einzelnen Generationen ist der gerade beschriebene Effekt aber so gering, dass auch in diesem Fall für die Untersuchung der Bedeutung von Kindern und ihrer angemessenen Berücksichtigung im Rahmen der GRV wiederum auf das Fixbeitragssatzverfahren verwiesen werden kann.

Bei abnehmender Wachstumsrate des Umfangs der Aktivgeneration steigt der Beitragssatz zur RV für die Mitglieder der Aktivgeneration an. Diese Beitragssatzsteigerung hat zur Folge, dass der ,Wert' eines Kindes mit gegebenem rentenversicherungspflichtigen Bruttoeinkommen (c.p.) bei gegebenem durchschnittlichen Bruttoeinkommen der Mitglieder der Aktivgeneration steigt. Zur Analyse der Bedeutung eines Kindes für die GRV kann hier auf den schon analysierten Fall eines ,Beitragssatzverfahrens' der GRV verwiesen werden, wobei hier intergenerational unterschiedliche Beitragssätze angesetzt werden müssen. Systematische Änderungen der dort getroffenen Aussagen ergeben sich hierdurch nicht. ${ }^{165}$

${ }^{164}$ Strenggenommen ist die Aufteilung der Bevölkerung in Aktive und Rentner relevant - nicht das Alter der jeweiligen Personen.

${ }^{165}$ Zum Problem der zu wählenden Diskontrate bei intertemporalen Änderungen des Beitragssatzes vgl. Abschnitt 4.3.3.2.1. 


\subsubsection{Fazit}

Der Ansatz einer Lebensstandardabsicherung im Alter hat in seinen wesentlichen Punkten analytisch keine besonderen Eigenschaften bezüglich der Bedeutung und angemessenen Berücksichtigung von Kindern. Seine hervorstechende Eigenschaft liegt vielmehr darin, dass in Abhängigkeit von der demographischen Entwicklung eine extreme Beitragsbelastung der Aktivgeneration nicht systemimmanent verhindert wird. Was theoretisch keine Probleme bereitet, wird praktisch zu einer Gefährdung des Rentenversicherungssystems durch ,Flucht' der Aktiven aus der Versicherung führen. Eine besondere Problematik des Systems besteht darin, dass bei intertemporal konstantem Absicherungsniveau Änderungen des ,Investitionsverhaltens' der Mitglieder der Aktivgeneration nicht zu Änderungen in der Höhe ihrer rentenversicherungsmäßigen Absicherung führen. Die Mitglieder der jeweiligen Aktivgeneration haben einen systematischen Anreiz, die quantitative Humankapitalbildung (Zahl der Kinder) zugunsten einer verstärkten ,qualitativen' Humankapitalbildung einerseits und einer gesteigerten Sachkapitalbildung andererseits einzuschränken. Die Begründung hierfür ist, dass das durchschnittliche Pro-Kopf-Einkommen der Aktivgeneration über die durchschnittliche Rentenhöhe der Mitglieder der Elterngeneration entscheidet, und nicht die Einkommenssumme aller Mitglieder der Aktivgeneration. Eine Steigerung des Pro-Kopf-Einkommens der Aktiven ist aus Sicht der Rentner (ökonomisch) attraktiver als eine Steigerung der Einkommenssumme der Aktiven infolge einer größeren Zahl von Erwerbstätigen.

Das gerade Ausgeführte ist allerdings in zweierlei Hinsicht abzuschwächen: Zum einen führen bei ,nettoorientierter' Lebensstandardabsicherung die steigenden Belastungen der Mitglieder der Aktivgeneration bei sinkender Wachstumsrate der Aktivbevölkerung (c.p.) zu einer Senkung des durch die RV abgesicherten absoluten Lebensstandards (auch) der Alten. Zum anderen ist in der bisherigen Darstellung implizit unterstellt worden, die durch das generative Verhalten induzierte höhere Belastung der Mitglieder der Kindergeneration mit Rentenverpflichtungen werde von der Elterngeneration nicht weiter beachtet - zumindest nicht über die Frage hinaus, ob sie nicht den Widerstand der Beitragszahler derart erhöht, dass die Rentenansprüche infolge fehlender ,Zahlungsbereitschaft' sukzessive unsicher resp. uneintreibbar werden; vielmehr wird nur die Rückwirkung der der nächsten Generation aufgebürdeten Belastung auf die zu erwartenden Renten berücksichtigt. Allerdings ist nicht auszuschließen, dass zumindest Eltern die Belastungswirkungen des Rentenversicherungssystems für ihre Kinder bei ihren Entscheidungen nicht gänzlich unberücksichtigt lassen. ${ }^{166}$ Letzteres kann sich insbesondere in privaten Transfers von Eltern hin zu ihren Kindern manifestieren. Bei den kin-

${ }^{166}$ Wobei die Anonymität des Rentenversicherungssystems allerdings - wie schon erwähnt - eine gewisse Tendenz hin zu vergleichsweise hohen Rentenansprüchen der Alten fördert. 
derlosen Alten ist hingegen nicht von einer solchen Bereitschaft zu ,Opfern' zugunsten der nachfolgenden Generation auszugehen. Obendrein reicht die Information der Eltern über die durch das Rentenversicherungssystem ihren Kindern aufgebürdete Last regelmäßig nicht aus, selbst bei ,bestem Willen' einen intergenerativ angemessenen Lastenausgleich vorzunehmen. Von bestehenden Belastungstendenzen ist deshalb unbedingt auszugehen. Insbesondere beeinflussen die erörterten Einschränkungen nicht die systeminduzierte individuelle Rationalität einer Substitution von ,Kinderzahl' durch ,Kapital'. 


\section{Die distributiven Konsequenzen einer (partiellen) Internalisierung der externen Effekte des Kinderhabens im Rahmen einer umlagefinanzierten Zwangsrentenversicherung}

Die bisherigen Ausführungen haben theoretisch vorstellbare Ausgestaltungsmöglichkeiten eines umlagefinanzierten Rentenversicherungssystems (mit Zwangsmitgliedschaft) analysiert, und hierbei insbesondere den ,Wert' von Kindern innerhalb des jeweiligen Systems einerseits und Möglichkeiten seiner Berücksichtigung untersucht. Im Folgenden soll ein Analyserahmen zur partiellen Internalisierung externer Effekte von Kindern im Rahmen einer umlagefinanzierten Rentenversicherung vorgestellt werden. Eine vollständige Internalisierung ist nicht möglich, wenn - wovon hier ausgegangen wird - das Ziel einer kollektiven Absicherung der Altersversorgung nicht aufgegeben wird: Insbesondere die Absicherung gegen Altersarmut als Folge einer niedrigen Einkommensposition der Kinder (z.B. infolge eines frühzeitigen Tods von Kindern, sehr geringem Einkommen der Kinder etc.) hat zur Folge, dass auch Familien Vorteile aus einer Rentenversicherung ziehen. Somit ist ein vollständiger Ausgleich der externen Effekte, wie sie sich für eine risikofreie Welt (oder in einer Ex-post-Perspektive) darstellen, nicht geboten.

Zuerst wird auf grundsätzliche Anforderungen an eine derartige Ausgestaltung resümierend eingegangen, um dann daraus einen Analyserahmen zu entwickeln. Anschließend werden die Eigenschaften des Systems näher vorgestellt, um dann ,Weiterentwicklungsmöglichkeiten' aufzuzeigen, sowie - allerdings nur ansatzweise - zu untersuchen, inwieweit es Grundlage einer praktikablen Ausgestaltung der Rentenversicherung (unter realitätsnäheren Bedingungen) sein kann. Außerdem wird im Rahmen des vorliegenden Kapitels thematisiert, welche Diskontrate zur Angleichung des Barwertes von in unterschiedlichen Perioden erfolgenden Zahlungen zu nutzen ist.

\subsection{Annahmen, Anforderungen und Ausgestaltungselemente}

Das im Folgenden konstruierte ,Referenzsystem' dient ausschließlich der Untersuchung der Bedeutung von Kindern für eine kollektiv organisierte Alterssicherung, d.h. alle in der Realität mit einer Rentenversicherung verbundenen Elemente der Absicherung von Hinterbliebenen, sowie einer Absicherung des Versicherten (und der von ihm wirtschaftlich Abhängigen) im Falle vorzeitiger Erwerbsunfä- 
higkeit werden ausgeblendet; auf sie wird später (vgl. 4.3.3.3) kursorisch eingegangen. Die betrachtete Rentenversicherung ist in einem strengen Umlageverfahren organisiert. Transaktions- und Verwaltungskosten werden nicht berücksichtigt.

Die Mitglieder der Aktivgeneration leisten zugunsten der Versichertengemeinschaft zwei Arten von Beiträgen: Zum einen Einzahlungen, zum anderen die ,Einbringung' von Kindern, die ihrerseits - in der nachfolgenden Periode - Beitragszahlungen leisten. Der Wert der Kinder für die Rentenversicherung zeigt sich in der Höhe ihrer späteren Beitragszahlungen, während der Wert der Beitragszahlungen der Mitglieder der Aktivgeneration ohne weiteres erkennbar ist.

Für die Kinderberücksichtigung wird im weiteren von einer Ex-post-Perspektive ausgegangen: Die Höhe aller relevanten Beitragszahlungen (auch der Kinder!) ist zur Zeit der Rentenberechnung bekannt. (Die Berechnung der monatlichen kinderbezogenen Rente erfolgt also erst am Ende der Aktivzeit der Kinder.) Da eine Versicherung der Individuen gegen das ,Risiko' der Langlebigkeit (bzw. gegen mit dieser verbundene finanzielle Engpässe) erfolgen soll, werden monatliche Zahlungen der Rentenversicherung an die noch lebenden Mitglieder der Rentnergeneration betrachtet. ${ }^{167}$

Bei der gerade geschilderten rentenmäßigen Berücksichtigung ist zu beachten, dass unterschiedliche Kinder in Abhängigkeit von ihren Beitragsleistungen für die Gemeinschaft der Rentenversicherten einen unterschiedlichen ,Wert' haben. Werden, wie in der vorliegenden Arbeit der Fall, zwecks Aufdeckung grundlegender realökonomischer Zusammenhänge ökonomische Analysekategorien auf das generative und erzieherische Verhalten der Eltern angewandt, ${ }^{168}$ so ist zu fordern, dass zwecks Vermeidung einer ,Überinvestition' in ,Kinderquantität' (Zahl der Mitglieder der Kindergeneration) und einer Unterinvestition in ,Kinderqualität'

${ }^{167} \mathrm{Da}$ der untersuchte Ansatz der Aufdeckung von Leistungen Versicherter zugunsten der Rentenversicherung dient, ist die Ex-post-Betrachtung geboten. Da andererseits eine Versicherung gegen Langlebigkeit erfolgen soll, d.h. eine Absicherung des Lebensstandards für die Rentenphase, unabhängig von deren (individueller) Länge, wăre es unangemessen, aus der Tatsache eines bekannten Wertes der Kinder für die Rentenversicherung auf die Sinnhaftigkeit einer zum Barwert der kumulierten Beitragszahlungen der Kinder proportionalen Einmalzahlung an die jeweiligen Rentner zu schließen, denn hierbei wăre keine ,Lebensstandardsicherung' Langlebiger mehr gegeben.

${ }^{168}$ Wie schon einleitend zum Ausdruck gebracht, wird in dieser Arbeit von einer solchen Perspektive ausgegangen, auch wenn damit nicht beansprucht werden soll, dass es angemessen ist, das Kinderhaben ausschließlich so zu sehen und dass eine Berücksichtigung weiterer Gesichtspunkte nicht andere (u.U. im Extremfall sogar gegengerichtete) Schlussfolgerungen gebietet. 
(durchschnittliche Einkommenshöhe der Mitglieder der Kindergeneration während ihrer Aktivzeit) Unterschiede in den Beiträgen der Kinder zur Rentenversicherung zu einer unterschiedlichen Höhe der Rentenansprüche ihrer Eltern führen. ${ }^{169}$

Da sowohl die Erziehung von Kindern, die in ihrer Aktivphase der Rentenversicherung beitreten als auch die eigenen Einzahlungen einen eigenständigen Beitrag zum Funktionieren der Rentenversicherung darstellen, sollen beide ,Leistungen' für sich allein genommen ausreichen, einen eigenständigen Altersrentenanspruch zu begründen. Somit werden sowohl kinderlose Beitragszahler als auch Eltern beitragszahlender Kinder, die selber keine Beiträge geleistet haben, grundsätzlich für das Alter finanziell abgesichert.

Der beitragsbezogene Rentenanspruch steigt mit der Summe der Beitragszahlungen in der Aktivzeit, der kinderbezogene Rentenanspruch mit der Summe der Beitragszahlungen der Kinder des Versicherten.

Auf die Vor- und Nachteile eines ,Splitting' des kinderbezogenen Rentenanspruchs zwischen Eltern- und Schwiegereltern wurde bereits eingegangen (vgl. 3.3.2.4.6.3). Für die weitere Betrachtung wird kein solches Splitting unterstellt. ${ }^{170}$ Hingegen wird die ,kinderbezogene Rente' auf die beiden Elternteile eines Kindes gleichmäßig aufgeteilt.

\subsection{Der Analyserahmen}

Der nachfolgende Abschnitt dient der zusammenfassenden Darstellung einer Ausgestaltung einer im Umlageverfahren finanzierten Rentenversicherung, die die gerade geforderten Eigenschaften hat. Die Präsentation erfolgt ,schrittweise': An eine verbale Erläuterung schließt sich eine formale Darstellung an. In beiden Fällen wird auf eine detaillierte Betrachtung des ,beitragsbezogenen Teils' verzichtet.

169 Sollten die Unterschiede zwischen den Versicherten rein zufällig sein, d.h. nicht auch Folge endogener Entscheidungen, so ist eine nicht erfolgende Berucksichtigung unterschiedlicher Beitragszahlungen (der Eltern und Kinder) allokativ unproblematisch (Sie entspricht dann einer ,Versicherung' gegen unterschiedliche Merkmalsausprägungen resp. ,Zustände der Welt'.). Für die weitere Untersuchung wird dieser - unrealistische - Fall jedoch nicht weiter beachtet.

${ }^{170}$ Die kinderbezogenen Rentenwerte entsprechen im Falle eines solchen ,Splittings' ungefähr der Hälfte des im weiteren jeweils pro Elternteil ausgewiesenen Wertes - dafur erhălt der Rentner dann aber auch eine kinderbezogene Rente fur Schwiegerkinder. 
Für diesen Teil wird implizit eine recht weitgehende Anlehnung an die gegenwärtig in Deutschland gültigen Regelungen unterstellt. ${ }^{171}$

\subsubsection{Die Aufteilung der Rente in einen kinder- und einen beitragsbezoge- nen Teil im Konkreten}

Die folgenden Eigenschaften kennzeichnen den betrachteten Ansatz:

1. Das Beitragsaufkommen wird durch eine zum rentenversicherungspflichtigen Einkommen der jeweiligen Mitglieder der Aktivgeneration proportionale Rentenversicherungsabgabe aufgebracht (Beitragssatzverfahren).

2. Die Beitragssumme wird unterteilt in einen ,beitragsbezogen' zu verteilenden $\left(\alpha_{t}\right)$ und einen ,kinderbezogen' zu verteilenden Anteil $\left(1-\alpha_{t}\right)$ (wobei $\left.\alpha_{t} \in[0 ; 1]\right)$.

3. Die Aufteilung des beitragsbezogenen Teils erfolgt grundsätzlich entsprechend der bisherigen Regelung im Rentenrecht (ohne Berücksichtigung von Kindern bzw. Erziehungszeiten). Die Annahme lässt sich damit begründen, dass in diesem Teil - definitionsgemäß - Kinder ohne besondere Bedeutung sind, so dass der Verzicht auf detailliertere Hinweise zur Ausgestaltung für das Thema der Arbeit unproblematisch ist. Außerdem hat das deutsche Rentenrecht die - im Sinne intragenerationaler (relativer) Beitragsäquivalenz der Höhe der monatlichen Rentenzahlung wünschenswerte - Eigenschaft, dass derjenige, der - im Vergleich mit einer Referenzperson - Zeit seines Erwerbslebens ein doppelt so hohes rentenversicherungspflichtiges Einkommen bezogen hat, auch - cum grano salis - eine doppelt so hohe beitragsbezogene Rente erhält. ${ }^{172}$

${ }^{171}$ Für die Aufteilung der beitragsbezogen zu verteilenden Einnahmen der Rentenversicherung ist hierbei die relative Einkommensposition (bezüglich des rentenversicherungspflichtigen Arbeitseinkommens) des Versicherten in den jeweiligen Jahren seiner Erwerbstătigkeit entscheidend - und nicht die absolute Höhe des jeweiligen Einkommens.

${ }^{172}$ Abweichungen im gegenwärtigen deutschen Rentenrecht sind Folge von Zurechnungen (Ausbildungszeiten), Hőherbewertungen von Zeiten mit niedrigem Beitrag (z.B. Lehre, aber auch generelle Hőherbewertung niedriger Beitrăge bei langer Beitragsdauer) etc. Alle diese Verrechnungen, die die Rendite normaler Beitragszahlungen schmälern (so nicht ein Ausgleich der mit ihnen verbundenen Kosten durch einen Bundeszuschuss erfolgt), werden hier nicht weiter be- 
4. Im kinderbezogenen Teil des Rentensystems wird der (ex-post bestimmte) rentenmäßige Barwert der einzelnen Kinder des Versicherten als Bezugsgröße genommen. ${ }^{173}$ Für den Versicherten erfolgt eine Addition der ,rentenmäßigen Werte' aller seiner Kinder. Da ein Splitting des kinderbezogenen Rententeils auf beide Elternteile vorgenommen wird, entspricht der dem jeweiligen Rentner zurechenbare ,Wert' seiner Kinder der Hälfte der gerade beschriebenen Summe. Dieser Wert wird durch die Summe der Barwerte der Beitragszahlungen aller Kinder von Rentnern dieser Periode dividiert. Der sich so ergebende Anteil des ,Wertes der Kinder' des Versicherten am ,Gesamtwert' der nachfolgenden Generation entspricht dem Anteil der kinderbezogen aufzuteilenden Rentensumme, der dem jeweiligen Versicherten zufließt. Die sich hierbei über alle Rentner ergebende Summe ,kinderbezogener' Renten stellt die unkorrigierte Summe der kinderbezogenen Renten dar.

5. Die unter 4. errechnete Summe ist zu ,korrigieren', da nicht für alle Kinder, von denen ein Elternteil lebt, auch der zweite Elternteil lebt. Deshalb wird durch die bisher vorgestellte Prozedur nicht die gesamte Summe der kinderbezogen aufzuteilenden Renten , ausgeschöpft'. Die auf die Verstorbenen (oder anderweitig nicht zu einem Rentenbezug Berechtigten) entfallenden Mittel werden nicht ,genutzt' - mit der Folge einer ,Unterausschöpfung'. Zur ,Ausschöpfungskorrektur' werden die individuellen kinderbezogenen Renten solange parallel erhöht, bis ihre Summe gerade dem zur Verfügung stehenden (kinderbezogen aufzuteilenden) Gesamtbetrag entspricht.

6. Aus der Addition seiner ,beitragsbezogenen' und seiner korrigierten ,kinderbezogenen' Rente errechnet sich die jeweilige Gesamtrente des einzelnen Rentners.

In formaler Hinsicht ergeben sich bei einer Realisierung dieser Merkmale folgende Zusammenhänge:

$$
B_{t} \equiv \beta_{t} \cdot \sum_{i} y_{t}^{n, i}
$$

Gleichung 4-1

trachtet. Im beitragsbezogenen Teil der Rentenversicherung wird - durch Herausnahme der ,kinderbezogenen Teile' - die Beitragsbezogenheit erhöht.

${ }^{173}$ An dieser Stelle wird auf die Problematik der Berechnung des Barwerts zukünftiger Beitragszahlungen nicht weiter eingegangen; eine Thematisierung erfolgt in Abschnitt 4.3.3.2.1. 
Die Beitragseinnahmen $\left(B_{t}\right)$ ergeben sich durch die Erhebung eines Beitragssatzes $\left(\beta_{t}\right)$ auf das rentenversicherungspflichtige Einkommen der jeweiligen Aktivgeneration (das der Summe der individuellen rentenversicherungspflichtigen Einkommen der Mitglieder der Aktivgeneration ( $\left.y_{t}^{r, i}\right)$ entspricht).

$$
\widetilde{K} \widetilde{R}_{t}^{j} \equiv\left(1-\alpha_{t}\right) \cdot B_{t}\left\{\frac{\text { Barwert der BZ der Kinder des Rentners }}{\text { Barwert der BZ aller Kinder aller gegenwärtigen Rentner }} \cdot \frac{1}{2}\right\} .
$$

\section{Gleichung 4-2}

Die unkorrigierte kinderbezogene Rente des einzelnen Rentners $\left(\widetilde{K} \widetilde{R}_{t}^{j}\right)$ berechnet sich als das Produkt des kinderbezogen zu verteilenden Teils des Beitragsaufkommens mit dem individuellen Anteil des jeweiligen Rentners. Dieser wiederum entspricht dem Anteil des Barwerts der Beitragszahlungen (BZ) der Kinder des Rentners am Barwert der Beitragszahlungen aller Kinder der Rentner der jeweiligen Periode. Es erfolgt eine Halbierung des so errechneten Wertes, da für jedes Kind beide Elternteile rentenberechtigt sind (Splitting der kinderbezogenen Rente).

$$
K R_{t}^{j} \equiv \widetilde{K} \widetilde{R}_{t}^{j} \cdot \frac{\left(1-\alpha_{t}\right) \cdot B_{t}}{\sum_{t} \widetilde{K} \widetilde{R}_{t}^{t}}
$$

(mit $\imath:$ Index der Rentner).

Für die ,Korrektur' des individuellen kinderbezogenen Rentenanspruchs wird das (etwa infolge verstorbener Elternteile) eingesparte Geld den verbleibenden kinderbezogenen Rentenansprüchen proportional ,zugeschlagen'. Das Resultat ist eine Vollausschöpfung der für die kinderbezogenen Renten vorgesehenen Finanzmittel.

$G R_{t}^{j} \equiv B R_{t}^{j}+K R_{t}^{j}$

Gleichung 4-4

Die Gesamtrente eines Rentners $\left(G R_{t}^{j}\right)$ ergibt sich aus der Addition seines beitrags- und seines (korrigierten) kinderbezogenen Rentenanspruchs ( $B R_{t}^{j}$ resp. $\left.K R_{t}^{j}\right)$. 


\subsubsection{Versicherung der Eltern gegen geringe Einkommen ihrer Kinder?}

Die ,angemessene' Berücksichtigung des finanziellen Beitrags der Kinder zur Rentenversicherung bietet - jenseits der Problematik der Diskontierung - einen Diskussionspunkt: Der untersuchte Ansatz hat zur Folge, dass das rentenversicherungspflichtige Einkommen des Kindes betreffende Schocks zu entsprechenden Änderungen auch der Höhe der kinderbezogenen Rente der Eltern führen. Solche Ereignisse können z.B. früher Tod bzw. Behinderung des Kindes, aber auch eine Krise in der von ihm gewählten Berufssparte, Branche etc. sein. Derartige Härten sind eindeutig kein Spezifikum des diskutierten Ansatzes. Gegen den Rückgang der Höhe der kinderbezogenen Rente infolge einiger der angesprochenen Ursachen (Tod, schwere Behinderung etc.) ließe sich zwar im Rahmen der Rentenversicherung eine Absicherung vornehmen, ohne damit allokativ schädliche Anreize zu setzen, doch stehen gerade diese Ereignisse auf Grund ihrer Eigenschaften einer individuellen Absicherung auf freien Versicherungsmärkten (in Form von ,Leibrenten') offen: ${ }^{174}$ Eine recht gute Möglichkeit der Kontrolle eventueller erheblicher Einschränkungen der Erwerbsfähigkeit erschwert Versicherungsbetrug; da nur ,gravierende Ereignisse', die Folge von Unfall, schwerer Erkrankung und Tod sind, versichert werden, ist die Bedeutung asymmetrischer Information gering. Die großen physischen und psychischen Belastungen des Opfers (und / oder seiner Eltern) machen - wenn zwischen den Familienmitgliedern (positive) Nutzeninterdependenz besteht, was als nicht allzu restriktive Annahme gelten kann eine bewusste Herbeiführung des Versicherungsfalls (sei es durch die Eltern, sei es durch das Kind) oder auch nur wesentliche Verhaltensänderungen, die seine Eintrittswahrscheinlichkeit erhöhen (moral hazard), wenig attraktiv. Als Folge der guten Bedingungen für ein Zustandekommen eines Marktes für Versicherungen besteht also keine in Marktversagen begründete Notwendigkeit einer zwangsweisen institutionellen Absicherung von Eltern.

Da hingegen, wo ein solcher privater Versicherungsmarkt nicht zustande kommt, d.h. insbesondere für den Fall ungewöhnlich niedriger (resp. hoher) Einkommen des Kindes im Vergleich mit anderen Kindern ähnlicher Qualifikation etc., ist gerade davon auszugehen, dass infolge adverser Selektion und moral hazard eine Versicherung (sowohl des Kindes als auch - über die Rentenversicherung - seiner Eltern) negative allokative Effekte hätte und von daher problematisch wäre.

174 Die beiden bedeutendsten Ausnahmen sind einerseits ungewollte Kinderlosigkeit, andererseits eine dauerhafte - mit Erwerbsunfähigkeit einhergehende - Behinderung von Kindern von Geburt an. In beiden Fällen dürfte eine marktliche Versicherung kaum möglich sein. Es ist aber wie bereits mehrfach erwăhnt - fraglich, ob für derartige Fälle eine ,Versicherung' im Rahmen der GRV erfolgen sollte, oder ob die Solidarleistung nicht von der Gemeinschaft der Steuerzahler finanziert werden sollte. 


\subsubsection{Zu weiteren Eigenschaften}

Zum ,Kennenlernen' des vorgestellten Ansatzes werden nun einige seiner Eigenschaften dargestellt.

- Da Rentenzahlungen nur aus monetären Beitragsleistungen erbracht, Rentenansprüche aber auch durch Beitragsleistungen der Kinder begründet werden, führt der Ansatz zu einer Schmälerung der Rendite der Beitragszahlungen gegenüber einem ausschließlich beitragszentrierten Ansatz. Die Zahlungen zur Rentenversicherung haben individuell stärkeren ,Steuercharakter'. Dies wiederum mag Anreizwirkungen auf das Arbeitsangebot, aber auch auf die Bereitschaft zum Ausweichen in die Schattenwirtschaft (sowie in Richtung Selbstständigkeit oder ,Auswanderung') haben. ${ }^{175}$ Diesem verstärkten Steuercharakter der direkten Beitragszahlungen steht jedoch ein verringerter Steuercharakter für Kinder zu erbringenden Aufwendungen gegenüber. Allerdings werden die Kosten der Kinderberücksichtigung offen gelegt. Dies mag von den ,Arbeitsanreizwirkungen' her nachteilig sein - für die Betonung der Bedeutung der Kindererziehung für eine umlagefinanzierte Rentenversicherung ist diese Offenheit jedoch vorteilhaft.

- Der Ansatz berücksichtigt nicht die bloße Zahl der Kinder eines Versicherten, sondern deren Beitragszahlungen zur GRV.

- Die hier vorgestellte hälftige Aufteilung des kinderbezogenen Rentenanspruchs auf beide Elternteile führt nicht zu der vielfach - so auch vom Bundesverfassungsgericht in seinem ,Rentenurteil' vom 07.07.1992 - ${ }^{176}$ geforderten Verbesserung der relativen Rentenposition von Elternteilen, die sich besonders um die Erziehung von Kindern gekümmert und dafür ihre Erwerbstätigkeit eingeschränkt haben. Da sich die Eltern die Kosten der Kindererziehung grundsätzlich ,teilen' (beide Partner sind vom Einkommensverzicht und von den zusätzlichen Kosten durch die Kindererziehung betroffen), erscheint eine hälftige Zurechnung der kinderinduzierten Rente auf beide Elternteile auch begründbar. Mit einer entsprechenden Argumentation lässt sich allerdings auch - ohne dass dies in der vorliegenden Arbeit aufgrund der unterbleibenden Darstellung des ,beitragsbezogenen' Rententeils deutlich wird

${ }^{175}$ Eine entsprechende Kritik trifft allerdings jeden Ansatz, der Kinder rentensteigernd berücksichtigt; es liegt folglich kein spezieller Nachteil einer konkreten Ausgestaltung vor, denn die ,kinderbezogenen' Renten(teile) werden immer aus monetären Beiträgen finanziert werden, und somit deren Rendite (c.p.) verringern.

176 Vgl. Anhang 5 (Abschnitt 7.5). 
- eine hälftige Zurechnung der beitragsinduzierten Rente auf beide Ehepartner vornehmen.

Eine Abkehr von der ,Halbteilungsregel' ist obendrein innerhalb des dargestellten Analyserahmens ohne systematische Probleme möglich, und führt nur zu äußerst geringfügigen Änderungen der Summe der Rentenansprüche. Innerhalb funktionierender Partnerschaften dürfte die Bedeutung der jeweiligen Aufteilung der - in ihrer kumulierten Höhe gegebenen - Rente auf die beiden Partner vergleichsweise gering sein, da eine innerfamiliäre Korrektur dieser Einkommensverteilung problemlos möglich ist. Sie bekommt allerdings dann Gewicht, wenn die Aufteilung einer gegebenen Rentensumme auf die beiden Ehepartner im Falle des Todes eines der Rentenberechtigten (oder der Scheidung etc.) für die Höhe des Rentenanspruch des anderen Ehepartners bedeutsam ist (und eben nicht ausschließlich die Summe der Rentenansprüche). Da in der allgemeinen Diskussion Bestrebungen zu einer ,eigenständigen' Alterssicherung beider Ehepartner regelmäßig mit Überlegungen zur Kürzung oder Abschaffung der Hinterbliebenenrente verbunden sind, ${ }^{177}$ hat die angesprochene Problemlage durchaus eine aktuelle politische Relevanz.

- Als Beitragssatzverfahren berücksichtigt der vorliegende Ansatz Preisniveauänderungen, so sie sich in der Höhe der rentenversicherungspflichtigen Einkommen niederschlagen - bei einer Parallelentwicklung der Preis- und Lohnindizes erfolgt also eine vollständige Absicherung der Rentnergeneration gegen Inflation, die Realwerte der Beitrags- und der Rentenzahlungen - sowie die relative Einkommensposition der Beitragszahler resp. Rentenempfänger bleiben unverändert. ${ }^{178}$

- Der ,Sozialisationserfolg' der Eltern manifestiert sich an der relativen Beitragsposition ihrer Kinder.

- Ändert sich die Summe des rentenversicherungspflichtigen Einkommens der Aktivgeneration, so partizipiert die Rentnergeneration daran proportional. Wie oben dargelegt, hat dies z.B. zur Folge, dass der relative Lebensstandard

177 Vgl. z.B. AlBERS (1997), BORCHERT (1993, S. 271) und die begeisterte Würdigung des Ansatzes BORCHERTs durch OETER (1989a).

178 Wobei sich hier die Frage nach dem richtigen Preisindex stellt: Eine Alterung der Gesellschaft kann eine uberdurchschnittliche Preissteigerungsrate gerade fur speziell von Alten nachgefragte Guter und Dienstleistungen zur Folge haben, so dass eine Rentensteigerung nach der ,allgemeinen' Preisniveauånderungsrate durchaus mit einer realen Absenkung des Lebensstandards der Rentner einhergehen kann. 
der Rentner steigt, wenn sich die Summe der Einkommen der Mitglieder der Aktivgeneration geringer erhöht als der Umfang der Aktivgeneration - und sinkt, wenn sich die Summe auf eine geringere Zahl von Mitgliedern der Aktivgeneration verteilt (also z.B. gleiche Einkommenssumme bei geringerer Kopfzahl, also gestiegenem Pro-Kopf-Einkommen). Die individuelle ,Zurechnung' des Sozialisationserfolgs des einzelnen Rentners erfolgt durch Variation seines Anteils an der kinderbezogen aufzuteilenden Rentensumme. ${ }^{179}$ Es liegt somit eine Kombination individueller und kollektiver Elemente der Absicherung vor: Kollektiv wird die insgesamt zur Rentengewährung zur Verfügung stehende Summe aufgebracht - ihre Aufteilung auf die Rentenberechtigten bestimmt sich nach deren individuellen Beiträgen während der Aktivzeit, wobei eine gesonderte Betrachtung monetärer und generativer Beitragsleistungen erfolgt.

Das gerade über die relative Einkommensposition der Rentner Gesagte lässt sich auch auf Änderungen in der Summe des rentenversicherungspflichtigen Einkommens, die durch Änderungen des generativen Verhaltens induziert werden, übertragen. Änderungen des Generationenumfangs, die zu proportionalen Änderungen der Summe des Einkommens der nachfolgenden Generation führen, werden insoweit, generativ internalisiert'. Interessanter ist aber ein möglicher Zusammenhang zwischen dem generativen Verhalten der Aktivgeneration und der Höhe der Renten der jeweiligen ,Ruhestandsgeneration': Ändert sich (c.p.) auf Grund veränderten generativen Verhaltens die Summe des rentenversicherungspflichtigen Einkommens der Aktivgeneration (z.B. infolge erhöhter Erwerbstätigkeit auf Seiten der Frauen), so partizipiert die Rentnergeneration automatisch am erhöhten Beitragsaufkommen. ${ }^{180}$ Die RV unterscheidet nicht nach den Ursachen einer erhöhten Einkommenssumme der Aktivgeneration. Diese Eigenschaft kann als allokativ nachteilig angesehen werden - doch ist sie eindeutig kein Spezifikum des untersuchten Analyserahmens.

179 Die minimale Ausweitung der individuellen Rentenhöhe aufgrund höherer Beitragszahlungen des eigenen Kindes ist hierbei zu vernachlässigen.

${ }^{180}$ Dies unterscheidet den betrachteten Ansatz von dem an einer Absicherung eines am durchschnittlichen Erwerbseinkommen eines abhängig Beschäftigten orientierten Verfahren: Bei letzterem würde eine Ausweitung der Beschäftigtenzahl, die mit einer Senkung des durchschnittlichen Lohnes einhergeht, zu einer entsprechenden Senkung der Rentenhöhe führen! 


\subsection{Verteilungsanalyse}

Im nachfolgenden Abschnitt soll simuliert werden, wie sich ein den vorangegangenen Anforderungen genügendes Umlageverfahren (für verschiedene Grade der ,Beitragsorientierung', die sich im jeweiligen Wert von manifestieren) ${ }^{181}$ distributiv auswirkt: Mit der Darstellung der distributiven Wirkungen der Kinderberücksichtigung werden (implizit) auch die distributiven Folgen einer Nicht-Berücksichtigung von Erziehungsleistungen verdeutlicht. Der eigentlichen Untersuchung gehen einige methodisch-technische Vorbemerkungen voran.

\subsubsection{Grundsatzfragen bei der Festlegung der empirischen Datenbasis}

Die zur Untersuchung benötigten Daten (Einkommen, Kinderzahl, Einkommen der Kinder) werden schon heute im wesentlichen von der GRV erfasst. Insoweit sind mit dem Ansatz keine besonderen Probleme einer Beschaffung der empirisch relevanten Daten verbunden. Dies heißt aber nicht, dass die Daten bereits, verknüpft' vorliegen - hier fehlen Informationen. Trotzdem soll der Versuch einer Abschätzung der Auswirkungen der Implementierung des Systems auf die Rentenhöhe unter der Annahme eines gegebenen Beitragsaufkommens unternommen werden.

\subsubsection{Basisdaten}

Die Rentensumme der Rentenversicherung der Arbeiter und Angestellten in Westdeutschland betrug im Jahre 1994 ca. DM 222 Mrd. Im selben Jahr gab es (ca.) 15,426 Mio. Rentenfälle. Bei der Berechnung der durchschnittlichen Rente ist zu berücksichtigen, dass in dieser Zahl Fälle enthalten sind, bei denen eine Versorgung der Hinterbliebenen von Versicherten erfolgt. Dabei wird regelmäßig nur ein prozentualer Anteil der vom jeweiligen Verstorbenen erworbenen Rentenansprüche ausgezahlt - unter (teilweiser) Anrechnung der anderweitigen Einkommen der Hinterbliebenen. Für die Berechnungen hier wird pauschalierend unterstellt, die Hinterbliebenenrente betrage 60 v.H. der Regelaltersrente des Verstorbenen. ${ }^{182}$ Davon ausgehend ergibt sich auf Basis der Zahl der bezogenen Renten im Jahre 1994 in Westdeutschland eine Zahl von ca. 13,68 Mio. ,standardisierten Renten-

${ }^{181}$ Im Folgenden wird aus Grlinden der Übersichtlichkeit auf einen Zeitindex verzichtet.

${ }^{182}$ Dieser Verhältniswert entspricht dem der ,großen Witwen-/ Witwerrente' im gegenwärtigen deutschen Rentenrecht. 
fällen' (Rentenfällen mit ,normalen Altersrenten'). ${ }^{183}$ Bei Nutzung der Nettoreproduktionsrate von 0,94 (Wert von 1955) als Repräsentant der durchschnittlichen Kinderzahl pro Mitglied der heutigen Rentnergeneration errechnet sich ein Umfang der Kindergeneration von ca. 12,86 Mio. Der ,Wanderungssaldo' zu anderen Systemen der sozialen Sicherung wird hier gleich 0 gesetzt. ${ }^{184}$ Bei der Bezugnahme auf die gegenwärtige Rentensumme ist zu beachten, dass diese in Teilen über einen Bundeszuschuss zur Rentenversicherung aufgebracht wird. Es wird für die weitere Untersuchung unterstellt, der Bundeszuschuss decke genau die versicherungsfremden Leistungen der Rentenversicherung. Folge dieser Annahme ist, dass bei der folgenden Analyse, die eine ausschließliche Beitragsfinanzierung der Renten unterstellt, die Höhe der monatlichen beitragsbezogenen Renten (in Höhe des Beitragsaufkommens ohne Bundeszuschuss) richtig ausgewiesen wird.

\subsubsection{Test auf Kalibrierung (des beitragsbezogenen Teils)}

Vor der eigentlichen Simulation wird der zugrundeliegende Modellrahmen erst einmal einem einfachen Test unterworfen: Der auf Basis der oben angegebenen Zahlen und reiner Beitragsorientierung ( ) errechnete Wert einer Durchschnittsrente darf sich - soll die Untersuchung zu ,realitätsnahen' Ergebnissen führen (können) - nicht wesentlich von dem statistisch ausgewiesenen Wert einer Durchschnittsrente im gegenwärtigen Rentenversicherungssystem unterscheiden.

Die bei der Abschätzung errechnete monatliche (durchschnittliche) Gesamtrente, die hier der beitragsbezogenen Rente entspricht, beträgt (ca.) DM 1.352. Im Vergleich dazu betrug die durchschnittliche Arbeiterrente in den alten Bundesländern im Jahre 1993 DM 1.054/ Monat und die durchschnittliche Angestelltenrente DM 1.469 / Monat. ${ }^{185}$ Gewichtet man die jeweiligen durchschnittlichen Renten

${ }^{183}$ Die Abschätzung basiert auf der ausgewiesenen Zahl von Rentenfällen (Rentenbestand) der Rentenversicherungen der Arbeiter resp. Angestellten, wobei die jeweilige Zahl der Witwenrenten mit dem Faktor 0,6 gewichtet wird (vgl. BUNDESMINISTER FÛR ARBEIT UND SOZIALORDNUNG (1995), S. 126f.; eigene Berechnung). Der so ermittelte Wert ist auf zwei Nachkommastellen gerundet.

${ }^{184}$ Es gibt auch Kinder die zur Rentenversicherung Beiträge leisten, deren Eltern aber heute nicht Renten innerhalb des Rentenversicherungssystems beziehen. Für diese Kinder entsteht im vorliegenden Analyserahmen ein Rentenanspruch. Allerdings ist zu beachten, dass ein Teil der Kinder der heutigen Rentnergeneration, der in der obigen Abschätzung enthalten ist, ihrerseits keine Beiträge zur Rentenversicherung leistet, und dementsprechend im betrachteten Analyserahmen auch keinen Rentenanspruch ihrer Eltern begründet. Im weiteren wird ein Ausgleich dieser beiden gegenläufigen Rentenpositionen unterstellt.

185 Vgl. STATISTISCHES BUNDESAMT, Statistisches Jahrbuch für Deutschland 1994 (1994), Tab. 19.4.10, S. 491. 
mit den Anteilen der Arbeiter bzw. Angestellten an allen (westdeutschen) Rentenbeziehern, so ergibt sich eine Durchschnittsrente von (ca.) DM 1.207 / Monat. ${ }^{186}$ $\mathrm{Zu}$ diesem Wert ist der Beitrag zur Krankenversicherung der Rentner zu addieren. Setzt man hierfür 13,0 v.H. an, ${ }^{187}$ so ergibt sich eine ,korrigierte Durchschnittsrente' von (ca.) DM 1.364 / Monat. Die Differenz zum simulierten Wert beträgt somit weniger als 1 v.H. und erscheint damit gering genug, um aus einem Vergleich der sich bei unterschiedlichen Parametern der Kinderberücksichtigung innerhalb des Ansatzes ergebenden jeweiligen Rentenhöhen realitätsnahe Aussagen über distributive Auswirkungen des Umlageverfahrens gewinnen zu können.

\subsubsection{Der ,Korrekturfaktor' und Probleme der Schätzung}

Die Schätzwerte für die Kinderzahl sind problembehaftet, da in der heutigen Rentnergeneration das Erwerbsverhalten innerhalb der Gruppe der Frauen erheblich divergiert. Ein nennenswerter Anteil der Frauen hat keinen eigenen (beitragsbezogenen) Rentenanspruch. Hieraus ergibt sich für die Abschätzung des Umfangs der Kindergeneration das Problem, dass bei vielen Kindern beide Elternteile einen eigenständigen Rentenanspruch haben, während dies bei anderen nur für einen Elternteil gilt. Für einige Kinder sind beide Elternteile erfasst, für andere nur ein Elternteil. Es ist bei der Abschätzung des Umfangs der Kindergeneration also mit deutlichen Ungenauigkeiten zu rechnen, wobei à priori nicht klar ist, ob eine Über- oder Unterschätzung erfolgt.

Das gerade Geschilderte erschwert die Schätzung des Korrekturfaktors (zur Vermeidung einer Unterausschöpfung) für den kinderbezogenen Teil der Rente. Ein einfacher Ansatz besteht darin, auf Basis der aktuellen Sterbetafeln für Westdeutschland die bedingten Erwartungswerte für die Zahl der lebenden Elternteile unter der Voraussetzung, dass mindestens ein Elternteil lebt, ${ }^{188}$ grob zu schätzen.

Unterstellt man stochastische Unabhängigkeit aller rentenrelevanten Parameter (insbesondere Unabhängigkeit der Lebenserwartung vom kumulierten rentenversi-

${ }^{186}$ Vgl. STATISTISCHES BUNDESAMT, Statistisches Jahrbuch fur Deutschland 1994 (1994), Tab. 19.4.2, S. 488; eigene Berechnung.

187 Der durchschnittliche Beitragssatz zur Krankenversicherung der Rentner betrug im Jahre 1993 bis zum 30. Juni 12,50 v.H., danach 13,40 v.H. (vgl. VERBAND DEUTSCHER RENTENVERSICHERUNGSTRÄGER (1999), S. 214). Der Jahresdurchschnitt lag mithin bei ca. 13,0 v.H.

${ }^{188}$ Lebt kein Elternteil mehr, so gibt es auch keinen diesbezüglichen Rentenanspruch. Dies spiegelt sich aber in der Zahl der Rentenfalle wider, und ist an dieser Stelle nicht mehr zu berücksichtigen. 
cherungspflichtigen Einkommen der Kinder und von der Höhe der Rente), so lässt sich grundsätzlich die ,Ausschöpfungsquote' - und dementsprechend der Korrekturfaktor zur Sicherung der Vollausschöpfung des kinderbezogenen Rentenvolumens - grob abschätzen. Für das Folgende wird unterstellt, das durchschnittliche Alter der Rentenbezieher betrage 65 Jahre. Die Wahrscheinlichkeit eines (lebendgeborenen westdeutschen) Knaben, dieses Alter zu erreichen, beträgt ca. 75 v.H., die eines Mädchens ca. 87 v.H. ${ }^{189}$ Legt man diese Zahlen zugrunde, ${ }^{190}$ so lässt sich der Erwartungswert der lebenden Elternteile unter der Bedingung, dass mindestens ein Elternteil lebt, mit 1,67 abschätzen (vgl. Anhang 3 (Abschnitt 7.3). Für die weitere Arbeit wird dann mit einem darauf basierenden ,Korrekturfaktor' von 1,30 gearbeitet (zur Abschätzung und zur Beziehung zum ,Erwartungswert der Zahl der lebenden Elternteile pro Kind (...)' vgl. ebenfalls Anhang 3 (7.3)). Wegen der mit der Abschätzung verbundenen Ungenauigkeit werden zusätzlich jeweils die Ergebnisse für den Fall einer , unkorrigierten Vollausschöpfung' (gekennzeichnet durch einen ,Korrekturfaktor' von 1,0) angegeben. ${ }^{191}$

\subsubsection{Simulation der rentenmäßigen Bedeutung von Kindern}

Im Folgenden werden verschiedene auf Basis des in Abschnitt 4.2 dargestellten Analyserahmens gewonnene Schätzergebnisse präsentiert. Bei der Interpretation der ausgewiesenen DM-Beträge ist zu beachten, dass diese die von den Rentenversicherungsträgern an die Krankenversicherung der Rentner zu leistenden Zahlungen inkludieren. Sie betragen mithin ca. das 1,13-fache des an den Rentner auszuzahlenden Betrags. Ferner ist zu beachten, dass die Abschätzung ,stationär' ist. Es gibt hier keine Rückwirkungen des ,beitragsbezogenen' Rentenanteils auf die Summe der Beitragszahlungen. Diese Annahme ist sicher für höhere Grade der Kinderorientierung des Rentenversicherungssystems unangemessen - immerhin bedeuten diese automatisch eine Senkung der (individuellen) ,Rendite' von Beitragszahlungen, verringern mithin die Arbeitsanreize, und dürften somit auf

189 Vgl. STATistisches BundeSAMT, Fachserie 1, Reihe 1, 1994, S. 167, Tab. 10.20.

190 Was nicht ganz angemessen ist: Die Überlebenswahrscheinlichkeit wird hierbei systematisch unterschätzt, da in der Berechnung nur Leute betrachtet werden, die Beiträge zur RV gezahlt und / oder Eltern geworden sind, also das Erwachsenenalter erreicht haben. Da andererseits aber das gewählte durchschnittliche Alter der Rentner eher etwas zu niedrig angesetzt ist, mit der Folge einer Überschätzung der Zahl der überlebenden Rentner, erscheint für eine überschlägige Rechnung der Verzicht auf weitere Korrekturen vertretbar.

191 Wie später deutlich wird, ist eine Schätzung für andere ,Korrekturwerte' einfach möglich, indem der unkorrigierte ,kinderbezogene' Rentenwert mit dem jeweiligen Korrekturfaktor multipliziert wird. 
das Aggregat ,Beitragseinnahmen der GRV' (verringernd), rückwirken'. Damit sind zwar per se keine distributiven Wirkungen innerhalb der Rentnergeneration verbunden, doch ist eine ,Überzeichnung ' der Rentenhöhe für hohe Grade der Kinderorientierung des Rentensystems zu unterstellen.

\subsubsection{Die absolute Höhe der unterschiedlichen Rentenkomponenten}

Zuerst soll der Einfluss des rentenversicherungspflichtigen Einkommens der Kinder auf die Höhe der Renten ihrer Eltern bei verschiedenen Werten von (Grad der Beitragsorientierung) untersucht werden. Es wird hier - wie in allen folgenden Analysen - ein Rentner betrachtet, der über sein Berufsleben hinweg ein für alle Rentner seiner Zeit ,typisches' rentenversicherungspflichtiges Einkommen hatte, also bei reiner Beitragsorientierung eine durchschnittliche monatliche Altersrente bezieht. ${ }^{192}$

Die sich hieraus ergebenden monatlichen Gesamtrenten und ,kinderbezogenen Renten' lassen sich den folgenden Darstellungen entnehmen. Der Wert der beitragsbezogenen Rente entspricht dem der Gesamtrente bei einer Kinderzahl von 0: Wer keine Kinder hat (oder nur Kinder, die keine Zahlungen zur GRV leisten), erhält ausschließlich aus dem ,beitragsbezogenen Rententeil' Leistungen. Die graphisch ausgewiesenen Werte sind die für den Fall einer erfolgenden Ausschöpfungskorrektur (mit dem Korrekturfaktor 1,30 für den kinderbezogenen Rententeil). ${ }^{193}$

Die folgenden Graphiken (Abbildungen 1 und 2) verdeutlichen, wie sich die beitragsbezogene Rente mit dem Ausmaß der ,Kinderorientierung' des Rentensystems ändert, und die kinderbezogene Rente mit dem Grad der Kinderorientierung des Rentensystems, aber auch in Abhängigkeit vom Barwert der kumulierten Beitragsposition der Kinder variiert. Die kumulierte Beitragsposition der Kinder ist hierbei als Anteil des Barwerts der Beitragszahlungen der Kinder des Versicherten an der Summe der Barwerte der Beitragszahlungen der Gesamtheit der Kinder aller Rentenbezieher zu verstehen. Ferner verdeutlichen die Abbildungen die Aus-

${ }^{192}$ Dies bedeutet nicht, dass der Betroffene in jedem Jahr seiner Aktivzeit ein durchschnittliches rentenversicherungspflichtiges Einkommen hatte: Die durchschnittliche Entgeltpunktzahl pro Versicherungsjahr betrug bei Altersrenten von Mănnern in den alten Bundesländern 1998 0,811 und bei Frauen 0,648 (vgl. VERBAND DEUTSCHER RENTENVERSICHERUNGSTRÄGER (1999), S. 99f.), und lag somit deutlich unter dem Wert von 1,00 im Falle eines regelmäßigen Bezugs eines für das jeweilige Jahr repräsentativen Einkommens.

${ }^{193}$ Sie betragen mithin das 1,3-fache der ,unkorrigierten' Werte. 
wirkungen der Beitragsposition der Kinder für die Gesamtrente (als Summe der beitrags- und der kinderbezogenen Rente) des betrachteten Rentners.

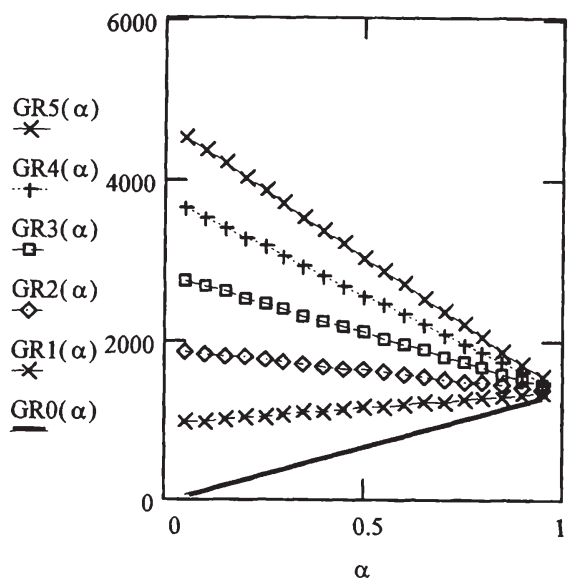

\begin{abstract}
Abbildung 1: Gesamtrente (korrigiert)
Lesehilfe: $\quad G R i(\alpha)$ - Gesamtrente bei einer kumulierten Beitragsposition der Kinder von $i$ und einem Anteil ,beitragsbezogener Renten' an der Rentensumme von $\alpha \cdot 100 \%$.
\end{abstract}

Den Graphiken ist zu entnehmen, dass ,Kinderreiche' von einer stärkeren Berücksichtigung des (individuellen) Sozialisationserfolgs profitieren, während Kinderlose eine erhebliche Verringerung ihres Alterssicherungsniveaus hinnehmen müssen. Aber auch Versicherte mit vergleichsweise geringer Kinderzahl (bzw. von Kindern mit niedrigen Beitragszahlungen) gehören - erwartungsgemä $\beta-z u$ den potenziellen ,Verlierern' einer ausgeprägten Kinderberücksichtigung im Rahmen der Rentenversicherung.

Außerdem ist aus den Graphiken ersichtlich, dass das Ziel einer Absicherung von Versicherten (auch) mit niedriger kumulierter Beitragsposition der Kinder bei niedrigen Werten der Beitragsorientierung nicht gegeben ist. Faktisch ist mit der Forderung einer (deutlichen) Absicherung auch Kinderloser eine restriktivere Ein- 
schränkung der unteren Grenze des Wertebereichs von $\alpha$ gegeben als mit der Forderung, der Wert müsse echt positiv sein. ${ }^{194}$

Beim Interpretieren des ,kinderbezogenen Rententeils' ist zu beachten, dass hier eine individualistische Betrachtung vorliegt. $\mathrm{Da}$ alle lebenden Elternteile (für das jeweilige Kind) eine gleich hohe kinderbezogene Rente erhalten, ergibt sich die (potenzielle) finanzielle Gesamtberücksichtigung der Kinder durch Verdopplung der oben ausgewiesenen Werte der kinderbezogenen Renten!

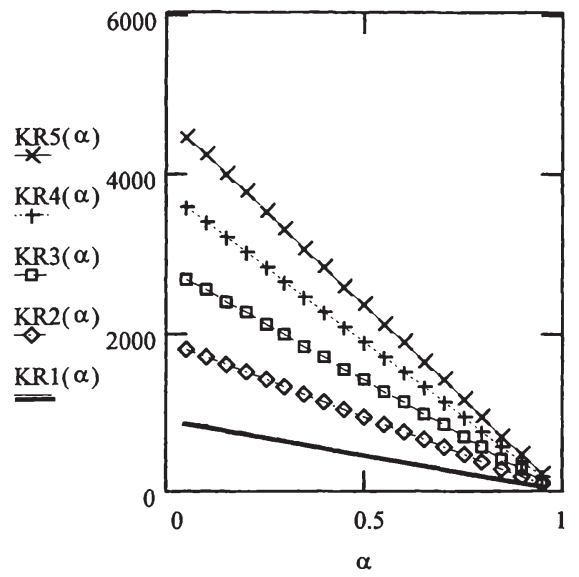

\section{Abbildung 2: Kinderbezogene Rente (korrigiert)}

Lesehilfe: $\quad K R i(\alpha)$ - Kinderbezogene Rente bei einer kumulierten Beitragsposition der Kinder von $i$ und einem Anteil ,beitragsbezogener Renten' an der Rentensumme von $\alpha \cdot 100 \%$.

${ }^{194}$ Entsprechendes gilt spiegelbildlich für die Forderung nach einer , ausreichenden' Absicherung kinderreicher Einkommensarmer - hier allerdings für eine Kappung der oberen Grenze des Wertebereichs. 
Tabelle 1 dient einer kurzen Darstellung wesentlicher Rentenwerte in Abhängigkeit vom gewählten Grad der Beitragsorientierung. Sie ergänzt mithin die vorangegangenen Graphiken und die genannten Werte, wobei hier auch die Werte ohne ,Ausschöpfungskorrektur' angegeben werden. (Es sei nochmals darauf hingewiesen, dass die ausgewiesenen DM-Beträge auch die zugehörigen Zahlungen der

\begin{tabular}{|c|c|c|c|c|c|c|c|c|c|}
\hline & $B R(\alpha)$ & \multicolumn{2}{|c|}{$K R 1(\alpha)$} & \multicolumn{2}{|c|}{$G R 1(\alpha)$} & \multicolumn{2}{|c|}{$K R 2(\alpha)$} & \multicolumn{2}{|c|}{$G R 2(\alpha)$} \\
\hline$\alpha$ & & korr. & unkorr. & korr. & unkorr. & korr. & unkorr. & korr. & unkorr. \\
\hline 0 & 0 & 935 & 719 & 935 & 719 & 1.870 & 1.439 & 1.870 & 1.439 \\
\hline 0,05 & 67 & 888 & 683 & 956 & 751 & 1.777 & 1.367 & 1.844 & 1.434 \\
\hline 0,10 & 135 & 842 & 647 & 977 & 783 & 1.683 & 1.295 & 1.818 & 1.430 \\
\hline 0,15 & 203 & 795 & 611 & 998 & 814 & 1.590 & 1.223 & 1.793 & 1.426 \\
\hline 0,20 & 270 & 748 & 575 & 1.019 & 846 & 1.496 & 1.151 & 1.767 & 1.421 \\
\hline 0,25 & 338 & 701 & 539 & 1.039 & 878 & 1.403 & 1.079 & 1.741 & 1.417 \\
\hline 0,30 & 406 & 656 & 504 & 1.060 & 909 & 1.309 & 1.007 & 1.715 & 1.413 \\
\hline 0,35 & 473 & 608 & 468 & 1.081 & 941 & 1.216 & 935 & 1.689 & 1.408 \\
\hline 0,40 & 541 & 561 & 432 & 1.102 & 973 & 1.122 & 863 & 1.663 & 1.404 \\
\hline 0,45 & 609 & 514 & 396 & 1.123 & 1.004 & 1.029 & 791 & 1.637 & 1.400 \\
\hline 0,50 & 676 & 468 & 360 & 1.144 & 1.036 & 935 & 719 & 1.611 & 1.396 \\
\hline 0,55 & 744 & 421 & 324 & 1.165 & 1.068 & 842 & 647 & 1.585 & 1.391 \\
\hline 0,60 & 811 & 374 & 288 & 1.185 & 1.099 & 748 & 575 & 1.560 & 1.387 \\
\hline 0,65 & 879 & 327 & 252 & 1.206 & 1.131 & 655 & 504 & 1.534 & 1.383 \\
\hline 0,70 & 947 & 281 & 216 & 1.227 & 1.162 & 561 & 432 & 1.508 & 1.378 \\
\hline 0,75 & 1.014 & 234 & 180 & 1.248 & 1.194 & 468 & 360 & 1.482 & 1.374 \\
\hline 0,80 & 1.082 & 187 & 144 & 1.269 & 1.226 & 374 & 288 & 1.456 & 1.370 \\
\hline 0,85 & 1.150 & 140 & 108 & 1.290 & 1.257 & 281 & 216 & 1.430 & 1.365 \\
\hline 0,90 & 1.217 & 94 & 72 & 1.311 & 1.289 & 187 & 144 & 1.404 & 1.361 \\
\hline 0,95 & 1.285 & 47 & 36 & 1.332 & 1.321 & 94 & 72 & 1.378 & 1.357 \\
\hline 1,00 & 1.352 & 0 & 0 & 1.352 & 1.352 & 0 & 0 & 1.352 & 1.352 \\
\hline
\end{tabular}

\section{Tabelle 1: Rentenwerte eines Rentners mit durchschnittlicher Beitragsposition in Abhängigkeit von $\alpha$}

Anmerkungen: Die Werte im Tabelleninneren sind auf volle DM gerundet. korr.: korrigiert, unkorr.: unkorrigiert; $B R$ : beitragsbezogene Rente bei durchschnittlicher Beitragsposition, $K R i$ : kinderbezogene Rente bei kumulierter Beitragsposition der Kinder von $i, G R i$ : Gesamtrente bei durchschnittlicher Beitragsposition und kumulierter Beitragsposition der Kinder von $i$. Die kumulierte kinderbezogene Rente für ein Kind mit ,durchschnittlicher Beitragsposition' ist dem entsprechenden Wert bei KR2 zu entnehmen (Wegen des ,Splitting' entspricht der hier ausgewiesene rentenmäßige Wert von Kindern mit kumulierter Beitragsposition von 2 dem kumulierten rentenmäßigen Wert eines einzelnen Kindes mit der Beitragsposition von 1.). 
GRV an die Krankenversicherung der Rentner inkludieren!) Die Angabe von Schätzwerten auch für sehr geringe Werte von $\alpha$ verdeutlicht, dass bei einer ,sehr geringen' Beitragsorientierung des Rentenversicherungssystems eine Absicherung für das Alter nur über ,Kindererziehung ' nicht möglich ist - und spiegelbildlich bei hohen Werten von $\alpha$ nicht ausschließlich durch Beitragszahlungen. Die Anforderung einer Absicherungsmöglichkeit ausschließlich durch Kindererziehung resp. Beitragsleistungen wird mithin in beiden Fällen nicht erfüllt. Sowohl zur Verdeutlichung dieses Sachverhaltes, als auch aus Gründen systematischer Klarheit wird aber auf eine Einschränkung des Wertebereichs von $\alpha$ schon an dieser Stelle verzichtet. ${ }^{195}$

\subsubsection{Substitution von Beitragszahlungen durch, generative Leistungen"}

In der Diskussion der Anforderungen an eine Kinderberücksichtigung im Rahmen der Rentenversicherung wurde gefordert, dass Beitragszahlungen durch das Heranziehen von Kindern, die ihrerseits Beiträge an die GRV abführen, substituiert werden können. Der folgende Teil der Untersuchung der (distributiven) Wirkungen des vorgestellten Ansatzes zur Kinderberücksichtigung zeigt zuerst für einen Grad der Beitragsorientierung des Rentenversicherungssystems, der durch $\alpha=0,50$ (bei ,Ausschöpfungskorrektur') gekennzeichnet ist, exemplarisch auf, welche Kombinationen von ,eigenen Beitragszahlungen' und (Summe der Barwerte der) ,Beitragszahlungen der Kinder' eine vorgegebene monatliche Höhe der (Gesamt-)Rente induzieren (vgl. Abb. 3). Anschließend werden die Substitutionsmöglichkeiten von beitragsbezogener durch kinderbezogene Rente in Abhängigkeit von $\alpha$ explizit angegeben (Abb.4). Auf eine entsprechende Darstellung für den Fall ohne Ausschöpfungskorrektur wird verzichtet. Es ergeben sich in prinzipieller Hinsicht keine neuen Einsichten. Da durch die Korrektur der Wert von ,kinderbezogenen Rentenleistungen' um 30 v.H. gesteigert wird, ist hier die Substitution von Beitragszahlungen durch ,Kindereinkommen' allerdings einfacher. Die Abbildungen verdeutlichen, dass der vorgestellte Ansatz die Forderung nach einer Substituierbarkeit von Beitragsleistungen durch Kindererziehung erfüllt. Die ,Schwierigkeit' einer Substitution hängt von der jeweiligen Beitragsorientierung ab: Je höher $\alpha$ ist, desto schwerer ist die Substitution von Beitragsleistungen durch die Heranziehung von (der GRV beitretenden) Kindern, es muss eine höhere kumulierte (relative) Beitragsposition der Kinder erzielt werden, um eine gegebene Beitragszahlung des Rentners zu ersetzen.

195 Ansonsten wäre schon an dieser Stelle festzulegen, welcher Wertebereich für $\alpha$ überhaupt ,zulässig‘ ist. Die Schätzwerte bei den jeweiligen $\alpha$ mögen aber für eine Festlegung des Wertebereichs hilfreich sein. 


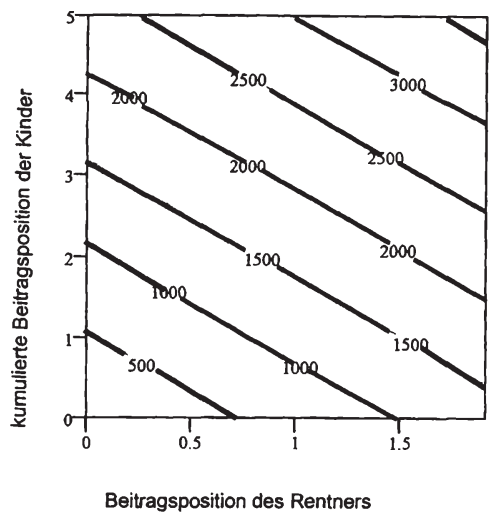

\section{Abbildung 3: Rentenhöhe (korrigiert) in Abhängigkeit von der Beitragsposition des Rentners und der kumulierten Beitragsposition seiner Kinder $(\alpha=0,50)$}

Lesehilfe: In der Abbildung werden Kombinationen von Beitragsleistungen und ,kumulierten Beitragspositionen der Kinder' aufgezeigt, die bei $\alpha=0,50 \mathrm{zu}$ gleich hohen Rentenansprüchen führen. So lässt sich eine monatliche Rente in Höhe von DM 1.000 allein durch Beitragszahlungen sichern, die beim ca. 1,5-fachen der durchschnittlichen Wertes liegen, oder aber allein durch Kinder, die zusammen ca. das 2,1-fache der durchschnittlichen Beitragszahlungen aller rentenrelevanten Kinder leisten.

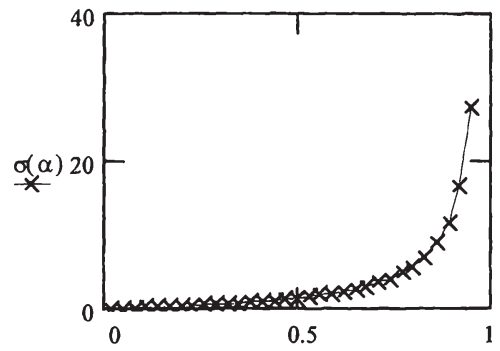

$\alpha$

Abbildung 4: Grenzrate der Transformation zwischen Beitragsposition des Rentners und der kumulierten Beitragsposition seiner Kinder in Abhängigkeit von $\alpha$ bei Ausschöpfungskorrektur

Hinweise: Der Analysebereich von $\alpha$ ist aus Gründen der Darstellung gegenüber dem zulässigen Bereich leicht eingeschränkt worden. Es erfolgt eine Wiedergabe des Betrags der Grenzrate der Transformation. Diese ist wie folgt definiert:

$$
\sigma(\alpha):=\left.\frac{\Delta \text { kumulierte Beitragsposition der Kinder }}{\Delta \text { Beitragsposition des Rentners }}\right|_{\Delta G R=0 ; \alpha} \text {. }
$$




\subsubsection{Zur Bestimmung von $\alpha$}

Die distributiven Effekte hängen entscheidend von dem für $\alpha$ vorgegebenen Wert ab. Für eine Bewertung der distributiven Wirkungen ist die Bestimmung des ,richtigen' Anteils der beitragsbezogen zu verteilenden Einnahmen der Rentenversicherung $(\alpha)$ deshalb von zentraler Relevanz. Die folgenden Abschnitte widmen sich diesem Problem.

\subsubsection{Hilfe durch Intuition?}

Ein naheliegender - und zu den früheren Erörterungen passender - Wert für $\alpha$ ist 0,50: Kinder (als spätere Beitragszahler) und Beitragseinnahmen sind gleichermaßen notwendig zur Aufrechterhaltung eines im Umlageverfahren ausgestalteten Rentenversicherungssystems. Problem dieser Argumentation, wie sie insbesondere von BORCHERT präsentiert wird (vgl. die noch folgenden Ausführungen zu seinem Rentenmodell in Abschnitt 5.1), ist, dass sowohl heutige Beitragszahlungen als auch spätere Beitragszahler zwar notwendig für die Existenz eines Rentenversicherungssystems sind. Aus dem Zusammenbruch des Systems beim Fehlen einer der beiden Komponenten kann aber nicht zwingend auf eine übereinstimmende Bedeutung in ,inneren Zuständen' des Systems geschlossen werden. ${ }^{196}$

\subsubsection{Ansatzpunkte einer systemimmanenten Abschätzung}

Im Folgenden soll der angemessene Grad der Beitragsorientierung des Rentenversicherungssystems nach dem Vorbild der unter (konkurrenz-)marktlichen Bedingungen erfolgenden Entlohnung von Produktionsfaktoren abgeschätzt werden. Hierbei wird ausschließlich auf die Alterssicherungsfunktion der umlagefinanzierten Rentenversicherung abgezielt.

${ }^{196}$ Zur Verdeutlichung hilft eine Bezugnahme auf die elementare Haushaltstheorie: Die CobbDouglas-Nutzenfunktion hat für den Fall zweier Güter die Form $U\left(x_{1}, x_{2}\right) \equiv c \cdot x_{1}^{\gamma} \cdot x_{2}^{1-\gamma}$ (mit $U:$ Nutzen, $x_{i}$ : Menge des Gutes $\left.X_{i}, \quad \gamma \in\right] 0 ; 1[, \mathrm{c}>0$ ). Obwohl $U\left(0, x_{2}\right)=U\left(x_{1}, 0\right)=0$ gilt, d.h. ein ,Minimalkonsum' beider Güter furr einen echt positiven Nutzen notwendig ist, erfordert die notwendige Bedingung für ein Nutzenmaximum nicht gleich hohe Ausgaben fur beide Guter. Es gilt vielmehr (bei einem nutzenmaximierendem Preisnehmer mit gegebenem Budget): $p_{2} \cdot x_{2}^{*}=\frac{!-\gamma}{\gamma} \cdot p_{1} \cdot x_{1}^{*}$ (mit: $p_{i}$ : Preis einer Einheit des Gutes $X_{i}$, wobei *, Optimalwerte' kennzeichnet). Damit reduziert sich der Fall gleich hoher Ausgaben für beide Produkte auf den Fall $\gamma=0,50$. Auf letzteren kann aber nicht aus der Existenz von Randnullstellen geschlossen werden! 
Die Mitglieder einer Generation tragen - wie schon betont - sowohl durch Beitragszahlungen als auch durch Kindeserziehung zum Erhalt der Rentenversicherung bei. Das Gut ,Erhalt der Rentenversicherung' wird mithin durch die beiden Produktionsfaktoren ,Beitragszahlungen' und ,Erziehung von Kindern, die Beitragszahlungen leisten' produziert. Der ,Wert' der ,Produktion' einer jeweiligen Periode entspricht der Summe der Beitragsleistungen der jeweiligen Periode zuzüglich dem (abdiskontierten) Wert der Beitragsleistungen der Mitglieder der Kindesgeneration. ${ }^{197}$ Der ,Produktionswert' der Periode $t\left(W_{t}\right)$ beträgt dann

$$
W_{t}\left(y_{t}^{r v}, N_{t+1}\right)=\underbrace{\beta_{t} \cdot N_{t} \cdot y_{t}^{r v}}_{\text {I }}+\underbrace{D_{t, t+1} \cdot \beta_{t+1} \cdot N_{t+1} \cdot y_{t+1}^{r v}}_{\text {II }} \quad \text { Gleichung 4-5 }
$$

wobei $D_{t, t+1}$ einen an dieser Stelle noch nicht weiter zu spezifizierenden Diskontierungsfaktor angibt (und $y_{t}^{r v}$ das durchschnittliche rentenversicherungspflichtige Einkommen eines Beitragszahlers in Periode $t$ ). Unter den hier vereinfacht dargestellten Bedingungen entscheiden die Mitglieder der gegenwärtigen Aktivgeneration über das eigene durchschnittliche rentenversicherungspflichtige Einkommen einerseits und die Zahl der Kinder andererseits.

Der in der ,Produktionsfunktion' mit $I$ gekennzeichnete Teil spiegelt den Wert der unmittelbaren Beitragszahlungen der Mitglieder der gegenwärtigen Aktivgeneration wider, der mit II bezeichnete Teil den Barwert der (indirekt) aus Kindererziehung resultierenden Leistungen von Mitgliedern dieser Generation. Wenn $\alpha$ nun dem Anteil der direkten Beiträge einer Generation zum Erhalt der Rentenversicherung entspricht, so lässt er sich (im hier gegebenen - restriktiven - Rahmen) folgendermaßen abschätzen:

${ }^{197}$ Diejenigen, die in der nachfolgenden Generation ihrerseits Kinder haben, die Mitglieder der Rentenversicherung werden, sichern den Fortbestand des Rentenversicherungssystems. Eine unmittelbare Leistung zugunsten der Elterngeneration leisten diese ,Enkel‘ jedoch nicht. 


$$
\alpha_{t}=\frac{\beta_{t} \cdot N_{t} \cdot y_{t}^{n}}{\beta_{t} \cdot N_{t} \cdot y_{t}^{r v}+D_{t, t+1} \cdot \beta_{t+1} \cdot N_{t+1} \cdot y_{t+1}^{r v}}=\frac{1}{1+D_{t, t+1} \cdot \frac{\beta_{t+1}}{\beta_{t}} \frac{N_{t+1}}{N_{t}} \cdot \frac{y_{t+1}^{r v}}{y_{t}^{r v}}} \cdot{ }^{198}
$$

\section{Gleichung 4-6}

Aus dieser Gleichung folgt (c.p., d.h. insbesondere für gegebenen Diskontierungsfaktor $\left.D_{t, t+1}\right): \frac{\partial \alpha_{t}}{\partial N_{t+1}}<0$ sowie $\frac{\partial \alpha_{t}}{\partial y_{t}^{N}}>0$, d.h. der Grad der Beitragsorientierung des Rentenversicherungssystems sollte abnehmen, wenn die Zahl der Mitglieder der Nachfolgegeneration (c.p.) zunimmt, und zunehmen, wenn (c.p.) das durchschnittliche rentenversicherungspflichtige Einkommen der Gegenwartsperiode steigt.

\subsection{Zum (richtigen) Diskontierungsfaktor in einem Umlageverfahren}

$\mathrm{Da}$ für die Bestimmung des ,richtigen' Wertes der Beitragsorientierung ein Vergleich der ,Wertigkeit' von Zahlungen aus verschiedenen Perioden erforderlich ist, werden im Folgenden nun unterschiedliche mögliche Diskontfaktoren vorgestellt und miteinander verglichen.

\subsection{Diskontierung mit dem Kapitalmarktzinssatz}

Eine mögliche Diskontierungsrate ist der Zinssatz einer langfristigen sicheren Geldanlage in der Währung, in der die spätere Zahlung erfolgt $\left(D_{t, t+1} \equiv \frac{1}{1+i_{t, t+1}}\right.$, mit $i_{t, t+1}$ : Kapitalmarktzinssatz für eine Geldanlage von Periode $t$ bis Periode $t+1)$. Vorteil dieses Wertes ist, dass er, zumindest auf den ersten Blick, gut beobachtbar erscheint - auch und gerade für lange Perioden. Außerdem ist einem Mitglied der Aktivgeneration eine langfristige Anlage zu diesem Zinssatz möglich, d.h. dieser Zinssatz verdeutlicht die Opportunitätskosten einer (Zwangs)Anlage von Geld in einer anderen Anlageform. Es ist allerdings zu beachten, dass die Rentenversicherung nicht nur eine ,Sparfunktion' zur Anlage von Geldern für den Alterskonsum hat, sondern auch andere ,Absicherungsfunktionen' wie etwa Leistungen bei Erwerbsunfähigkeit, Rehabilitation etc. Da die Rentenversicherung mithin ,Zusatzvorteile' gegenüber einer gewöhnlichen Geldanlage bietet, ist der

${ }^{198}$ An dieser Stelle erhält der Grad der Beitragsorientierung explizit einen Zeitindex um zu verdeutlichen, dass hier eine Schwankung des Wertes im Zeitablauf möglich ist. 
Vergleichszinssatz der Kapitalanlage entsprechend zu korrigieren (verringern), was die ,Zugänglichkeit' des Wertes erheblich schmälert.

\subsection{Diskontierung mit der Rate der , biologischen Verzinsung'}

Die wohl bekannteste Diskontrate für eine umlagefinanzierte Rentenversicherung basiert auf der ,biologischen Verzinsung' ( $D_{t, t+1} \equiv \frac{N_{t}}{N_{t+1}}$ ). Die (Netto-)Verzinsung entspricht hier bei intertemporaler Konstanz der Beitragszahlung gerade der (Netto-)Wachstumsrate der Zahl der in der RV versicherten Erwerbstätigen. ${ }^{199}$ Wird in nicht-stationären Umgebungen dieser Diskontierungsfaktor genutzt, so werden Preisniveauänderungen nicht erfasst, zukünftige (inflationierte) Zahlungen mithin überbewertet. Ferner ist eine entsprechende Diskontierung zu hinterfragen, wird es als Ziel der Rentenversicherung angesehen, den Lebensstandard der Rentner entsprechend dem der Aktiven zu ändern (Sicherung einer relativen Einkommensposition), oder sollen (indirekt) nutzenmäßige ,Belastungen' unterschiedlicher Generationen durch jeweilige Beitragszahlungen vergleichbar gemacht werden; denn auch in diesen beiden Fällen werden spätere Zahlungen bei ,biologischer Diskontierung', übergewichtet'.

\subsection{Diskontierung mit der Zuwachsrate des rentenversicherungspflichtigen Einkommens}

Ein weiterer Ansatz für die Bestimmung der Diskontierungsrate ist die Diskontierung mit dem Faktor des Zuwachses des rentenversicherungspflichtigen Einkommens $\left(D_{t, t+1} \equiv \frac{N_{t} \cdot y_{t}^{r v}}{N_{t+1} \cdot y_{t+1}^{r v}}\right)$. Vorteil einer derartigen Diskontierung ist die Berücksichtigung von Preisniveauänderungen, die sich in proportionalen Änderungen des durchschnittlichen rentenversicherungspflichtigen Einkommens niederschlagen. Allerdings werden hierbei auch über den Inflationsausgleich hinausgehende Änderungen des durchschnittlichen (rentenversicherungspflichtigen) Einkommens einbezogen; für die Rentenversicherung bedeutet dies wiederum, dass eine Partizipation der Rentnergeneration an Änderungen des Lebensstandards der Mitglieder der Aktivgeneration als ,normal' betrachtet und die höheren Zahlungen der Nachfolgegeneration quasi ,wegdiskontiert' werden; die Leistungen der Eltern, die diese ,produktiven' Kinder herangezogen haben, werden mithin vergleichsweise niedrig gewichtet.

199 Vgl. SAMUELSON (1958), insbes. S. $471 \mathrm{f}$. 


\subsection{Diskontierung mit der Zuwachsrate des Beitragsaufkommens}

Abschließend sei auf die Diskontierung mit dem Zuwachsfaktor des Beitragsaufkommens $\left(D_{t, t+1} \equiv \frac{\beta_{t} \cdot N_{t} \cdot y_{t}^{r v}}{\beta_{t+1} \cdot N_{t+1} \cdot y_{t+1}^{n}}\right)$ hingewiesen. Problem einer derartigen Diskontierung ist, dass sie die kumulierten Zahlungen der nachfolgenden Periode gerade so gewichtet, dass sie definitionsgemäß denselben Barwert haben wie die Beitragszahlungen der gegenwärtigen Periode. Das Ergebnis der Barwertberechnung wird mithin vorweggenommen.

\subsection{Vergleich der Diskontfaktoren}

Eine regelmäßig wünschenswerte Eigenschaft eines Diskontierungsfaktors kann es sein, unter (in der Realität anzutreffenden) nicht-stationären Umgebungsbedingungen zwei Zahlungen in unterschiedlichen Perioden, die sich gerade (prozentual) um ,die' Preisniveauänderungsrate unterscheiden, den gleichen Barwert zuzuordnen. Dies ist beim biologischen Diskontierungsfaktor gerade nicht der Fall: Ändert sich z.B. das Preisniveau von Periode $t$ nach Periode $t+1$, und wird die (durchschnittliche) Beitragszahlung zur Rentenversicherung entsprechend der allgemeinen Preissteigerungsrate angepasst, so wird hier der Wert der in Periode $t+1$ erfolgenden Zahlung überhöht ausgewiesen. Da in dem auf dem Kapitalmarkt verlangten Nominalzinssatz bereits eine ,Vergütung' für die erwartete Preissteigerung im involvierten Zeitraum enthalten ist, gibt es einen solchen Fehler hier (idealtypisch) nicht. Dies mag auf den ersten Blick für eine Nutzung des Kapitalmarktzinssatzes zur Diskontierung der Zahlungsströme sprechen. Eine solche Schlussfolgerung wäre jedoch voreilig: Ziel der ,Berechnungen “ ist es nicht, einen eventuellen Nachteil des Einzelnen aus der Tatsache der Pflichtbeitragszahlung zur Rentenversicherung zu bestimmen, sondern die angemessene Aufteilung innerhalb des Systems.

Wird nach dem realen, Wert' von Kindern innerhalb des Rentenversicherungssystems gefragt, so erscheint die ,rein biologische' Abdiskontierung ungeeignet; nicht die Zahl der Kinder, sondern deren Summe der Beitragszahlungen zur GRV entscheiden über die Rendite der Einzahlungen der Mitglieder der jeweiligen Aktivgeneration. Andererseits hat eine Diskontierung mit der Zuwachsrate des Beitragsaufkommens eine Vernachlässigung einer eventuellen Bereitschaft der nachfolgenden Generation, Mittel zur Unterstützung der Rentner aufzubringen, zur Folge. Dies wiederum kann kaum sinnvoll sein, wird die ,Bedeutung' der Kindererziehung für die Rentenversicherung, bezogen auf die Mitglieder der jeweiligen Aktivgeneration, betrachtet. 
Die Abdiskontierung mit der Zuwachsrate des rentenversicherungspflichtigen Einkommens kann hier einen Kompromiss darstellen: Es erfolgt eine Abdiskontierung auf Basis der Entwicklung der Bemessungsgrundlage, nicht aber eine Berücksichtigung, inwieweit diese Bemessungsgrundlage auch ,abgeschöpft' wird. ${ }^{200}$ Ferner ist in dieser Zuwachsrate eine Korrektur allgemeiner Preissteigerungstendenzen, die sich in Steigerungen des rentenversicherungspflichtigen Einkommens niederschlagen, enthalten. Es werden aber auch reale Einkommenssteigerungen ,nivelliert'. Dies gilt insbesondere auch für Änderungen der Abgrenzung des rentenversicherungspflichtigen Einkommens (z.B. infolge einer Einbeziehung weiterer Aktivitäten in die Rentenversicherungspflicht). Dies hat wiederum zur Folge, dass eine über die ,Inflationsrate ${ }^{201}$ hinausgehende prozentuale Ausweitung der Bemessungsgrundlage, die dazu dient, Einkommen von den Mitgliedern der Aktivgeneration an die Mitglieder der Rentnergeneration umzuverteilen (z.B. um letzteren eine Partizipation an einem steigenden Lebensstandard der Aktivgeneration zu ermöglichen), oder ein eventuelles Zurückbleiben hinter dem Wert, das einer gegenteiligen, Lastumschichtung' dient, bei einer Abdiskontierung mit der Änderungsrate des rentenversicherungspflichtigen Einkommen ausgeblendet wird.

Bei der Diskontierung mit der Änderungsrate des Beitragsaufkommens der Rentenversicherung verschärft sich das gerade bezeichnete Problem noch: Auch hier erfolgt - unter den schon beschriebenen Annahmen, und mit den ebenfalls schon beschriebenen Vor- und Nachteilen - eine ,Wegdiskontierung' von Änderungen des beitragspflichtigen Realeinkommens sowie der Einkommensänderung entsprechend der Inflationsrate, aber auch von intergenerativen Lastumschichtungen infolge veränderter Anteile rentenversicherungspflichtiger Einkommen am Gesamt(arbeits)einkommen eines repräsentativen Mitglieds der Aktivgeneration. Bei der Abdiskontierung auf Basis der Änderungsrate des Beitragsaufkommens wird aber zusätzlich auch noch der Einfluss eventueller Änderungen des Beitragssatzes auf die Höhe des Beitragsaufkommens neutralisiert. Da die Änderung des Bei-

${ }^{200}$ Hierbei kann allerdings das Problem einer ,Manipulation' der Bemessungsgrundlage nicht vermieden werden: So wird für den Fall, dass der Anteil des rentenversicherungspflichtigen Einkommens am Gesamteinkommen der Mitglieder der jeweiligen Aktivgeneration sinkt (z.B. weil sich die Mitglieder zunehmend der Rentenversicherungspflicht entziehen), nur eine geringe Abdiskontierung der zukünftigen Beitragsleistungen vorgenommen, und somit den Beiträgen der Kinder eine vergleichsweise hohe Bedeutung zuerkannt, obwohl die Kindergeneration realiter nur zu einer vergleichsweise geringen Tragung von Rentenlasten bereit ist.

201 Wobei auch die Wahl des Preisindex Auswirkung auf die intergenerative ,Lastverteilung' hat: Wird bei einer ,Überalterung' der Gesellschaft und damit einhergehender überproportionaler Erhöhung des Preisindexes des von den ,Alten' konsumierten Warenkorbes letzterer zur ,Inflationierung' der Renten (und somit der Beitragsverpflichtungen) genutzt, so erhőht das die Zahlungsverpflichtungen der Jungen gegenüber dem Fall einer Orientierung an der Änderung des Preisniveaus des von den Jungen konsumierten Güterbündels. 
tragssatzes wiederum - wie die der Bemessungsgrundlage - einer Änderung der intergenerativen Einkommens- resp. Lastverteilung dienen kann, erscheint die ,Wegdiskontierung' problematisch.

Eine eindeutige Antwort auf die Frage nach dem ,richtigen' Diskontierungsfaktor gibt es wohl nicht. Vielmehr hängt die richtige Wahl von der jeweiligen Fragestellung ab. So kann z.B. nicht der ,Wert' einer Zahlung eigentliches Erkenntnisobjekt sein, sondern indirekt der mit dieser Zahlung verbundene Nutzenentgang, oder aber die relative Belastung eines Aktivmitglieds durch die Beitragszahlung (Verhältnis zwischen Beitragszahlung und - verfügbarem - Einkommen): Die real höhere Beitragszahlung in einer späteren Periode induziert bei höherem Realeinkommen einen gleich hohen Nutzenentgang (bzw. die gleiche prozentuale finanzielle Belastung) wie eine entsprechend niedrigere Beitragszahlung zu einer Zeit mit niedrigerem Realeinkommen. ${ }^{202}$ Soll dies berücksichtigt werden, so erscheint die Nutzung eines auf der Änderungsrate des Einkommens basierenden Diskontierungsfaktors angemessen. Sollen jedoch ,nur' (intergenerationale) Finanzströme untersucht werden, so ist eine wie gerade beschrieben erfolgende Abdiskontierung nicht sinnvoll: Änderungen im ,realen' Wert der Beitragsleistungen nachfolgender Generationen sind für die Rendite der Beitragsleistungen eines einzelnen Mitglieds mitbestimmend; ihre ,Wegdiskontierung' wäre mithin falsch.

Bei Betrachtung der individuellen Rationalität einer freiwilligen Beitragszahlung zur Rentenversicherung ist eine Abdiskontierung zukünftiger Zahlungsströme mit dem Zinssatz einer vergleichbaren Alternativanlage, wie er auf dem Kapitalmarkt ermittelt wird (bei Berücksichtigung eines Abschlags für sonstige Versicherungsleistungen), angemessen. Begründung hierfür ist hier, dass so die Opportunitätskosten der (Zwangs-)Mitgliedschaft in der Rentenversicherung verdeutlicht werden. Es ist allerdings auch zu beachten, dass für eine kollektive Betrachtung von Zahlungsströmen die gerade geschilderte Wahl problematisch ist: Zum einen kann eine massenhafte ,Umschichtung' von Geldern auf den Kapitalmarkt Zinssatzänderungen induzieren, so dass eine kollektive Nutzung der ,Renditevorteile' einer kapitalmarktbasierten Altersabsicherung diese Renditevorteile möglicherweise abschwächt. Zum anderen - und dies ist der systematisch bedeutsamere Punkt würde ein massives Umschwenken der Mitglieder der Aktivgeneration eine anderweitige Aufbringung der Finanzmittel für die Rentnergeneration notwendig machen (z.B. eine höhere Besteuerung). Die Wahl der Rendite einer Kapitalmarktanlage als Referenzmaßstab klammert aus, dass eine umlagefinanzierte Rentenversicherung eben auch der Unterstützung der gegenwärtigen Rentnergeneration dient.

${ }^{202}$ Die Anfangs erwähnte Forderung, Zahlungen zweier Perioden, die denselben realen Wert haben, sollten auch gleich ausgewiesen werden, wird damit aufgegeben! 
Die erfolgte Diskussion unterschiedlicher Diskontierungsfaktoren hat verdeutlicht, dass die Wahl des ,richtigen' Diskontierungsfaktors einerseits von den Umgebungsbedingungen abhängt, zum anderen vom jeweiligen Erkenntnisinteresse. Damit aber die Wahl der Diskontrate bedeutsam für die folgenden Abschätzungen sein kann, müssen die Zinssätze voneinander abweichen. Dies ist bei den ,systemimmanenten' Diskontierungsfaktoren unter statischen Bedingungen (intertemporal übereinstimmende nominale Pro-Kopf-Größen bei Preisniveaustabilität) nicht der Fall, wie ein einfacher Blick auf die unterschiedlichen Diskontierungsfaktoren verdeutlicht. Ferner ist zu beachten, dass es unter einfachen neoklassischen Modellannahmen zu einer Angleichung des Zinssatzes auf dem Kapitalmarkt und des ,biologischen Zinssatzes' kommt, so dass auch hier eine Unterscheidung entbehrlich ist. ${ }^{203}$

\subsection{Abschätzung von $\alpha$ für verschiedene Diskontierungsfaktoren}

Im Folgenden soll der Grad der Beitragsorientierung $\left(\alpha_{t}\right)$ für die unterschiedlichen im vorangegangenen Abschnitt dargestellten Diskontierungsmethoden aufgezeigt werden.

\subsection{Abschätzung von $\alpha$ bei Diskontierung mit dem Kapitalmarktzinssatz}

Bei einer Diskontierung mit einem am Kapitalmarkt gebildeten Diskontierungssatz gilt: $D_{t, t+1} \equiv \frac{1}{1+i_{t, t+1}}$, wobei $i_{t, t+1}$ dem Vergleichszinssatz für die betrachtete Periode entspricht. Für Gleichung 4-6 folgt $\frac{\partial \alpha_{t}}{\partial i_{t,+1}}>0$; der Wert der Beitragsleistungen steigt relativ zum Wert der Kindererziehung, wenn die späteren Leistungen der Kinder zugunsten ihrer Elterngeneration stärker abdiskontiert werden.

${ }^{203}$ Zum Problem der ,richtigen' Diskontrate vgl. WERDING (1998), S. 484. Seine Ablehnung einer Nutzung der Verzinsung auf dem Kapitalmarkt begrlindet er (ebd., S. 249) damit, dass sich im Umlageverfahren ,die ,Verzinsung' .. technisch auf ganz andere Weise vollzieht“". Dieser technische Hinweis kann aber wohl kaum widerlegen, dass für den einzelnen Beitragszahler die Rendite am Kapitalmarkt (abzulglich eines, angemessenen' Abschlags für weitere Versicherungsleistungen) einen Referenzmaßstab darstellt. 
4.3.3.2.2.2. Abschätzung von $\alpha$ bei Diskontierung mit der Rate der , biologischen Verzinsung'

Bei einer Diskontierung mit der Rate der ,biologischen Verzinsung' $\left(D_{t, t+1} \equiv \frac{N_{t}}{N_{t+1}}\right)$ vereinfacht sich Gleichung 4-6 zu $\alpha_{t}=\frac{1}{1+\frac{\beta_{t+1}}{\beta_{t}} \cdot \frac{y_{t+1}^{r v}}{y_{t}^{r v}}}$. Für eine stationäre Umgebung ist intertemporale Konstanz des Beitragssatzes und des durchschnittlichen rentenversicherungspflichtigen Einkommens zu unterstellen (also: $\beta_{t}=\beta_{t+1}$ sowie $y_{t}^{r v}=y_{t+1}^{r v}$ ), wodurch sich die Gleichung weiter $\mathrm{zu} \alpha_{t}=\frac{1}{2}$ kürzen lässt. Der so ermittelte Grad der Beitragsorientierung entspricht dann gerade dem von BORCHERT als ,natürlich' angesehenen Wert. Für nicht-stationäre Umgebungen sind übereinstimmende rentenversicherungspflichtige Durchschnittseinkommen (auch bei Betrachtung des Realwertes) allerdings problematisch: Ihre Konstanz bedeutet (z.B.) ein Zurückbleiben der Rentnerabsicherung hinter Zuwächsen des Lebensstandards der Aktivgeneration.

\subsection{Abschätzung von $\alpha$ bei Diskontierung mit der Zuwachsrate des rentenversicherungspflichtigen Einkommens}

Bei einer Diskontierung mit dem Faktor des Zuwachses des rentenversicherungspflichtigen Einkommens $\left(D_{t, t+1} \equiv \frac{N_{t} \cdot y_{t}^{v}}{N_{t+1} \cdot y_{t+1}^{r v}}\right)$ gilt für den , angemessenen' Grad der Beitragsorientierung: $\alpha_{t}=\frac{1}{1+\frac{\beta_{t+1}}{\beta_{t}}}$. Er ist (c.p.) mithin um so niedriger, je mehr die nachfolgende Generation bereit ist, ihr Einkommen mit der Altengeneration zu ,teilen', also: $\frac{\partial \alpha_{t}}{\partial \beta_{t+1}}<0$. Für den Fall eines intertemporal konstanten Beitragssatzes $\left(\beta_{t}=\beta_{t+1}\right)$ zur Rentenversicherung ergibt sich wiederum $\alpha=\frac{1}{2}$.

\subsection{Abschätzung von $\alpha$ bei Diskontierung mit der Zuwachsrate des Beitragsaufkommens}

Erfolgt die Diskontierung mit dem Zuwachsfaktor des Beitragsaufkommens $\left(D_{t, t+1} \equiv \frac{\beta_{t} \cdot N_{t} \cdot y_{t}^{n v}}{\beta_{t+1} \cdot N_{t+1} \cdot y_{t+1}^{r v}}\right)$, so folgt daraus $\alpha_{t}=\frac{1}{2}$; dieser Wert ist auch intuitiv nach- 
vollziehbar, werden doch zukünftige Zahlungen der Kinder genau so abdiskontiert, dass sie denselben Barwert haben wie die Beitragszahlungen der gegenwärtigen Aktivgeneration.

\subsection{Hinweis auf besondere Werte von $\alpha$}

Abschließend soll - in Abweichung von der gerade präsentierten ,systemischen' Herleitung von Werten für den Grad der Beitragsorientierung - noch kurz auf die Implikationen zweier Extremwerte hingewiesen werden.

Der erste Wert lautet: $\alpha=0$. In diesem Fall bestimmen ausschließlich die Beitragszahlungen der eigenen Kinder die relative Höhe der Rente ihrer Eltern. Insoweit liegt hier eine kollektive Annäherung an ein System vor, das im Groben einer Alterssicherung im Kreise der Großfamilie entspricht: Dort bestimmen die Leistungen der Kinder den Lebensstandard ihrer Eltern im Alter. Der Unterschied besteht darin, dass bei $\alpha=0$ nur die relative Höhe der Rente der Eltern durch die Beiträge der eigenen Kinder determiniert wird, nicht aber die absolute. Diese folgt vielmehr aus dem Zusammenspiel von Gesamtbeitragsaufkommen aller Mitglieder der Aktivgeneration einerseits und dem Anteil der Kinder des jeweiligen Rentners daran, wird mithin unter dem Rückgriff auf ,kollektive' Größen ermittelt. Diese Kombination individueller und kollektiver Bestimmungsgrößen ist notwendig, um eine Absicherung der Rentner gegen besondere ,Langlebigkeit' (bzw. die mit dieser verbundenen Risiken) sowie eine Streckung der Beitragsbelastung der Kinder auf deren gesamte Aktivzeit zu erreichen; das letztere dient dazu, eine Konzentration der Belastung auf die Phase, in der die eigenen Eltern noch leben, zu vermeiden. Auf die (problematischen) Anreizwirkungen einer ausschließlichen Rentenbegründung durch Beitragsleistungen der Kinder ist bereits hingewiesen worden (vgl. 3.3.2.3.2).

Der zweite Wert lautet: $\alpha=1$. In diesem Fall beeinflussen ausschließlich die eigenen Beitragszahlungen die relative Höhe der Altersrente, während die kumulierten Beitragszahlungen der nachfolgenden Aktivgeneration über die absolute Höhe der Rente bestimmen. Es liegt hier eine recht weitgehende Annäherung an die Logik der gegenwärtig in Deutschland praktizierten Ausgestaltung der umlagefinanzierten Rentenversicherung vor. ${ }^{204}$ (Die (problematischen) Anreizwirkungen einer derartigen Ausgestaltung bezüglich Investitionen in das Humankapital sind bereits zu genüge thematisiert worden.)

${ }^{204}$ Abweichungen hieraus ergeben sich (im gegenwărtigen deutschen Rentensystem) durch die Rentengewährung für Kindererziehung, Höherbewertungen etc. 


\subsection{Abschätzung auf Basis der deutschen Rentenversicherung}

Abschließend sollen auf Basis von Daten des deutschen Rentenversicherungssystems die unterschiedlichen Grade der Beitragsorientierung (sehr) grob abgeschätzt werden. Hierfür wird in den Schätzern der Zeitraum von $1960(t)$ bis $1997(t+1)$ betrachtet. 1960 gab es (in Westdeutschland) ca. 18,79 Mill. abhängig Beschäftigte, die nicht Beamte waren (eine gesonderte statistische Erfassung ,sozialversicherungspflichtiger Beschäftigter' gab es damals nicht) ( $\left.N_{t}\right), 1997 \mathrm{ca} .22,14 \mathrm{Mill}$. sozialversicherungspflichtige Beschäftigte $\left(N_{t+1}\right){ }^{205}$ Der Beitragssatz zur Gesetzlichen Rentenversicherung der Arbeiter und Angestellten betrug 1960 14,0 v.H. $\left(\beta_{t}\right)$ und 1997 20,3 v.H. $\left(\beta_{t+1}\right){ }^{206}$ das (jährliche) Durchschnittsentgelt betrug DM 6.101 $\left(y_{t}^{r v}\right)$ bzw. $\left.53.806\left(y_{t+1}^{r v}\right)\right)^{207}$ Für die Verzinsung am Kapitalmarkt werden Nominalzinssätze von $6,0,7,0$ und 8,0 v.H. betrachtet.

Die Spannbreite der ausgewiesenen Schätzer $(\hat{\alpha})$ verdeutlicht die Bedeutung der

\begin{tabular}{|l|l|}
\hline Diskontierung mit & $\hat{\alpha}$ \\
\hline Kapitalmarktzinssatz 6 v.H. & 0,364 \\
\hline Kapitalmarktzinssatz 7 v.H. & 0,448 \\
\hline Kapitalmarktzinssatz 8 v.H. & 0,534 \\
\hline Wr. des Generationenumfangs & 0,073 \\
\hline Wr. d. rentenversicherungspflichtigen Einkommens & 0,408 \\
\hline Wr. des Beitragsaufkommens & 0,5 \\
\hline
\end{tabular}

Tabelle 2: Abschätzung des, richtigen' Grades der Beitragsorientierung für verschiedene Diskontierungsmethoden

Anmerkung: Wr: Wachstumsrate

Wahl des ,richtigen' Diskontierungsfaktors für die Bestimmung des angemessenen Grades der Beitragsorientierung der umlagefinanzierten Rentenversicherung. Aber selbst bei einem, Vergleichszinssatz ${ }^{6}$ von 8 v.H. wären die generativen Leistungen, d.h. die Beitragszahlungen der Kinder, fast genauso bedeutsam wie die

${ }^{205}$ Vgl. Verband DeUtSCHER RENTENVERSiCHERUNGSTRÄGER (1999), S. 219 \& 221.

${ }^{206}$ Vgl. VERBAND DEUTSCHER RENTENVERSICHERUNGSTRÄGER (1999), S. 213.

${ }^{207}$ Vgl. SGB VI Anlage 1 - fưr 1997 vorläufiges Durchschnittsentgelt. 
Beitragszahlungen! Die Zahlen spiegeln ferner wider, dass die Anlage von Geldern in der GRV eine ,attraktive' Anlageform war - insbesondere infolge der sehr hohen Wachstumsrate des durchschnittlichen Entgelts der abhängig Beschäftigten, wobei eben auch infolge der hohen Beitragsorientierung der gegenwärtigen Ausgestaltung der Rentenversicherung besondere ,Mitnahmemöglichkeiten' infolge ausschließlicher Beitragszahlungen zur GRV bestanden. (Die Zahlen lassen sich aufgrund der deutlichen Abschwächung der Zuwachsrate des rentenversicherungspflichtigen Einkommens eindeutig nicht als ,Prognosen' für zukünftige Perioden verwenden! ${ }^{208}$ )

\subsubsection{Folgerung}

Die vorangegangenen Rechnungen haben verdeutlicht, dass - bei einer ,produktionstheoretisch ${ }^{6}$ ausgerichteten Konzeption der Bestimmung von $\alpha$-die Wahl des Grades der Beitragsorientierung des Rentenversicherungssystems entscheidend von der Wahl des Diskontierungsfaktors abhängt. Dieser wiederum wird von der jeweiligen Fragestellung beeinflusst, wobei wiederum die Zielsetzungen des Rentenversicherungssystems zu berücksichtigen sind (so z.B., inwieweit die Altengeneration an Änderungen des Lebensstandards teilhaben soll, und ob eine eventuelle Teilhabe ,natürlich' ist, oder eine gesondert zu berücksichtigende Leistung darstellt). Bei der Festlegung des beitragsbezogen aufzuteilenden Anteils des Beitragsaufkommens sind aber über die gerade ausgeführten Punkte hinaus noch weitere Überlegungen geboten:

- Mit der Festlegung von $\alpha$ wird gleichzeitig über die Höhe des ,Steueranteils' der Beitragszahlungen entschieden. Die hieraus resultierenden Anreizwirkungen sind zu berücksichtigen und mit Anreizwirkungen hin zu Investitionen in das Humankapital der nachfolgenden Generation abzuwägen. Insbesondere ist zu bedenken, dass mögliche Anpassungsreaktionen auf dem Arbeitsmarkt sich im System weitaus kurzfristiger auswirken als die ,generativen'. Andererseits steigt im analysierten Ansatz mit dem rentenversicherungspflichtigen Einkommen der Kinder auch der monatliche Rentenanspruch der Eltern, was bei intrafamiliärer Nutzeninterdependenz zu einer Senkung des Anreizes zur Vermeidung von Beitragszahlungen auf Seiten der Kinder führen dürfte - zumal, wenn

${ }^{208}$ Im Zeitraum von 1960 bis 1997 stieg das Durchschnittsentgelt im Durchschnitt um ca. 6,1 v.H. jährlich; im Zeitraum von 1980 bis 1997 hingegen nur um durchschnittlich 3,6 v.H., und von 1992 bis 1997 nur um 2,8 v.H. 
Teile der zusätzlichen Rente im Rahmen einer intrafamiliären Einkommensumverteilung wieder zu den Kindern ,zurückfließen' sollten. ${ }^{209}$

- In der bisherigen Argumentation wurde ein eigenständiges ,Absicherungsziel' (,Sozialhilfevermeidungsfunktion') der GRV vernachlässigt. Ob eine derartige Ausklammerung in der Praxis angemessen ist, erscheint zweifelhaft. Wenn aber eine Absicherung sowohl durch ,durchschnittliche' Beitragszahlungen als auch durch die Erziehung von Kindern mit durchschnittlicher Summe von Beitragszahlungen erreicht werden soll, so grenzt dies den ,möglichen' Wertebereich von $\alpha$ weiter ein: Zwar lässt sich argumentieren, mit der Festlegung von $\alpha$ werde nur über Anteile entschieden, nicht aber über Niveaus. Bestehen allerdings Vorstellungen über das, richtige' Niveau der Rente beim Vorliegen bestimmter rentenrelevanter Umstände, und ist außerdem - sowohl wegen möglicher Anreizwirkungen, als auch infolge unerwünscht starker Liquiditätsbeschränkungen (in Verbindung mit einem unvollkommenen Kapitalmarkt) ein hoher Beitragssatz mit anderweitigen Nachteilen verbunden, so ist die Bestimmung von Anteilen und Niveaus nicht mehr getrennt zu sehen.

- Die Kosten von Kindern sind einerseits nicht per se rentenrelevant, andererseits findet eine Förderung des Kinderhabens bzw. eine Berücksichtigung der damit verbundenen Kosten bereits in anderen Systemen (Steuern, Sozialhilfe etc.) statt. Wird die ,Förderung' in diesen Systemen als nicht ausreichend angesehen (z.B. da das sich im Kinderfreibetrag manifestierende Existenzminimum von Kindern als in seiner Höhe als zu niedrig gilt), so sind diese Systeme - und nicht die Rentenversicherung - der richtige Ort zur Korrektur dieser Missstände. Man kann weitergehend argumentieren: Da Kinder ihren Eltern im Durchschnitt Nutzen stiften, also (auch) ,Konsumgutcharakter' haben, während Beitragszahlungen zur GRV ausschließlich den Charakter eines ,Investitionsgutes' besitzen, also nicht per se, sondern nur durch die von ihnen erwartete ökonomische Wirkung Nutzen stiften, ist eine gleichgewichtige Berücksichtigung von Kindern und Beiträgen auch von daher nicht geboten. Entsprechend mag auch wegen der größeren Vermeidungsanfälligkeit bzw. Steuerreagibilität von Beitragsleistungen gegenüber Humankapitalinvestitionen (Quantität und Qualität von Kindern) zur Vermeidung der Erosion der Beitragsbemessungsgrundlage eine asymmetrische Berücksichtigung erfolgen.

209 Die Argumentation, der zur Finanzierung kinderbezogener Rente herangezogene Teil der Beitragszahlungen der Aktiven werde von letzteren nicht als reine ,Steuerzahlung' verstanden, trägt allerdings sicher nicht mehr fur den Fall, dass keine Verwandten (denen ein ,Wohlergehen' gegönnt wird) leben. 
- Abschließend sei erneut darauf verwiesen, dass in den bisherigen Ausführungen ausschließlich auf den ,Alterssicherungsteil' der Rentenversicherung Bezug genommen wurde. Kommt es im Rahmen der Rentenversicherung zusätzlich auch zu einer Mittelverwendung zugunsten der Absicherung von (Halb-) Waisen (und von Halbwaisen betreuenden Elternteilen), ohne dass hierfür entsprechend höhere Beitragszahlungen von Versicherten mit wirtschaftlich abhängigen Kindern geleistet werden müssen, so stellt dies spezifische Leistungen des Rentenversicherungssystems zugunsten Kinderhabender dar. Sie sind bei der Festlegung des Grades der Beitragsorientierung im Altersrententeil zu berücksichtigen.

\subsubsection{Mögliche Erweiterungen sowie Praktikabilitätsdimensionen}

Im Folgenden werden verschiedene Eigenschaften des untersuchten Ansatzes noch einmal kritisch hinterfragt. Hierbei steht die Frage im Vordergrund, inwieweit aus dem vorgestellten Analyserahmen, der der Verdeutlichung externer Effekte der Kindererziehung im Rahmen einer umlagefinanzierten Rentenversicherung dient, Empfehlungen für eine Ausgestaltung eines kinderberücksichtigenden Umlageverfahrens gewonnen werden können.

Ein bedeutendes Problem ist in Schwierigkeiten einer Umstellung eines bestehenden Rentenversicherungssystems zu sehen. Insbesondere die $\mathrm{zu}$ unterstellenden Unterschiede in der Geschwindigkeit, in der Anpassungen im generativen Verhalten einerseits und im Arbeitsangebot andererseits erfolgen, können - schon aus Gründen des Vertrauensschutzes - eine ,behutsame' Umstellung erfordern. Eventuelle deutliche Absenkungen der Rentenhöhe (oder erwarteten Höhe der Rente) bei einem vergleichsweise schnell erfolgendem Umstieg können zwar durch faktische ,Bestandssicherungsklauseln', in denen eine Absenkung der Rentenhöhe infolge des Systemwechsels für eine erhebliche Zeitspanne ausgeschlossen wird, vermieden werden. Doch die Gewährung von Auffüllbeträgen zur Sicherung des alten Renten(anspruchs)niveaus bei sofortiger Umstellung (man vergleiche die Rentenanpassung in der ehemaligen DDR seit der deutschen Wiedervereinigung) hat, kommt es zu einer fundamentalen Umstellung des Rentenversicherungssystems, erhebliche Kosten zur Folge. Bei einer langsamen Umstellung besteht hingegen die Möglichkeit, Kürzungen der nominalen Renten(ansprüche) weitgehend zu vermeiden - dies geschieht aber auf Kosten einer nur schrittweise erfolgenden ,Höherbewertung' von Erziehungsleistungen. Aus allokativer Sicht mag eine solche Ausgestaltung allerdings den Vorteil haben, dass die Entscheidung über die Kinderzahl, aber auch über die elterliche Investition in ihre Kinder, häufig eher abgeschlossen ist als die Beitragszahlungsphase, so dass der kurzfristige Umfang der Kinderberücksichtigung im Rahmen der Rente mit Bezug auf das 
generative Verhalten ohne erhebliche Auswirkungen bleibt. Soweit eine langsame Einführung keinen Zweifel an der am Ende des Anpassungsprozesses stehenden Intensität der Kinderberücksichtigung aufkommen lässt, und außerdem die Versicherten die zukünftigen Verschiebungen der (relativen) Rentenhöhen antizipieren, setzt eine langsame Umstellung auch für die noch ,rentenfernen' Jahrgänge die richtigen ,Signale' bezüglich der zukünftigen rentenmäßigen Bedeutung des generativen Verhaltens. Die einschränkenden Annahmen sind allerdings gravierend: Insbesondere kann bezweifelt werden, dass weite Teile der Bevölkerung in der Lage sind, sich die Auswirkung einer veränderten Ausgestaltung des Rentenversicherungssystems auf ihre jeweilige Rentenposition in Abhängigkeit von ihrem generativen Verhalten vorzustellen. (Das Problem mag aber durch eine Information der Versicherten über ihre ungefähre Rentenhöhe bei sofortiger Gültigkeit der ,neuen' Berechnungsregeln gemildert werden.) Wird die Änderung der Gewichtung generativer im Vergleich zu monetären Leistungen jedoch nicht ,richtig' antizipiert, so hat dies zur Folge, dass auch eine (eventuelle) Anpassung des generativen Verhaltens verzögert wird.

Bei der weiteren Argumentation werden Probleme einer Umstellung des bestehenden Rentenversicherungssystems ausgeblendet, um praktische Probleme einer Ausgestaltung, die Folge des analysierten Ansatzes sind, herausarbeiten zu können.

Zuerst ist zu beachten, dass in der Analyse eine Ex-post-Betrachtung vorgenommen wurde. Die ,rentenrelevanten Daten' lagen somit allesamt vor. In der Realität ist dies im Hinblick auf die Beitragszahlungen der Kinder von Versicherten nicht der Fall. Es stellt sich somit die Frage, ob ein ,akzeptabler' zeitnaher Ersatz für die fehlenden Informationen besteht. Hierbei wäre an einen Rückgriff auf Informationen wie Ausbildung und bisherige Beitragszahlungen der Kinder der Versicherten zu denken, aus denen eine Abschätzung über die von den Kindern zukünftig noch ,zu erwartenden' Beitragszahlungen konstruiert wird. Eine solche Abschätzung hat allerdings einige bedeutsame Nachteile: Zum einen läuft sie auf eine partielle Pauschalierung der Kinderberücksichtigung im Rahmen der GRV hinaus, und geht dementsprechend mit gewissen ,Ungerechtigkeiten', wie sie mit jeder Pauschalierung verbunden sind, einher. Ferner setzt eine solche Pauschalierung regelmäßig allokativ fehlerhafte Anreize: So wird z.B. eine formale Qualifikation gefördert, wenn diese als Indikator vergleichsweise hoher zukünftiger Zahlungen zur GRV genutzt wird. Werden hingegen die Beitragszahlungen der Kinder bis zur jeweiligen Rentenzahlungsperiode für die Eltern als Grundlage für die Berechnung der kinderbezogenen Rente genommen, so bedeutet dies eine implizite Förderung von Berufen mit vergleichsweise flachem intertemporalen Ein- 
kommensprofil (bei gegebenem Lebenseinkommen), also z.B. eine Diskriminierung längerer Aus- und Weiterbildungszeiten. ${ }^{210}$

Sowohl bei pauschalierender Berücksichtigung zukünftiger Beitragszahlungen der Kinder, als auch bei ausschließlicher Bezugnahme auf die zum Zeitpunkt der Rentenzahlung an die Eltern schon geleisteten Beitragszahlungen der Kinder kommt es somit, wie verdeutlicht, zu per se unerwünschten Wirkungen. ${ }^{211}$ Diesem Nachteil ist allerdings der Vorteil einer im Durchschnitt gesteigerten Internalisierung der externen Effekte der Humankapitalbildung im Rahmen des Rentenversicherungssystems gegenzurechnen, so dass die Inkaufnahme einiger ,Ungenauigkeiten' gerechtfertigt sein kann. Immerhin setzt eine vollständige Pauschalierung der Kinderberücksichtigung im Rahmen der Rentenversicherung, wie sie zur Zeit in Deutschland praktiziert wird, ebenfalls allokativ falsche Anreize, stellt also keine best practice dar.

Wenn aber an dieser Stelle eine Pauschalierung als möglicherweise akzeptabel angesehen wird, dann bleibt $\mathrm{zu}$ fragen, ob - angesichts der mit ihr verbundenen Vorteile (vgl. 3.2.1.3) (und auch angesichts des Spürbarmachens in der Umstellungsphase) - eine Kinderberücksichtigung schon in der Beitragszeit möglich ist. In den bisherigen Erörterungen wurden bei der Kinderberücksichtigung auf Beobachtungen über rentenrelevante Eigenschaften des jeweiligen Kindes zurückgegriffen - im analytisch reinen Fall (Ex-post-Betrachtung) auf die kumulierten Beitragszahlungen des Kindes, in der gerade erfolgten Diskussion über eine ,praxisnähere' Ausgestaltung auf Beitragszahlungen des Kindes bis zum jeweiligen Berechnungszeitpunkt der Rente seiner Eltern, sowie auf mögliche beitragsrelevante Eigenschaften des Kindes, die sich zu diesem Zeitpunkt beobachten lassen (und als ,Indikatoren' der zu erwartenden Beitragszahlungen dieses Kindes dienen).

Bei einer Kinderberücksichtigung schon in der Aktivzeit vergrößern sich die Abschätzungsprobleme extrem, denn es müssen bedeutende Parameter schon in der Aktivzeit bekannt sein: Zuerst zu nennen ist der Erwartungswert des Barwerts der Summe der Beitragszahlungen in den jeweiligen ,Rentenperioden' der derzeit

210 Vom übereinstimmenden (rentenversicherungspflichtigen) Lebenseinkommen wird in diesem Fall ein größerer Anteil bei der Berechnung der kinderbezogenen Rente der Eltern berücksichtigt. Entsprechendes gilt auch fur Kinder, bei deren Geburt die Eltern jung waren.

211 Ob bei Berücksichtigung der tatsächlichen Beitragszahlungen ein Anreiz für Frauen besteht, eine eventuelle Erwerbsunterbrechung zugunsten der Kindererziehung für einen früheren oder späteren Zeitpunkt ihrer Aktivzeit ,einzuplanen', kann ohne weiteres nicht entschieden werden: Eine frühere Unterbrechung steigert (c.p.) die eigene spätere Rente, senkt aber die der eigenen Eltern ... 
Aktiven. Er gibt an, welchen, Wert' die gesamte Kindergeneration einmal in ihrer Aktivzeit haben wird. Zusätzlich wird noch ein ,Indikator' des Anteils des jeweiligen Kindes an dieser Beitragssumme benötigt, um somit seinen konkreten Wert für das Rentenversicherungssystems zu ,errechnen' ${ }^{212}$ Eine sofortige Kürzung der Beitragszahlung kindererziehender Versicherter hat (c.p.) eine Verringerung des Beitragsaufkommens und somit der Renten der gegenwärtigen Rentenbezieher zur Folge. Soll dies vermieden werden - und warum sollen die Rentner infolge einer Änderung der intertemporalen Allokation der Finanzmittel der Kindergeneration, die deren Barwert unverändert lässt, Rentenkürzungen hinnehmen? -, so muss der Beitragssatz zur Rentenversicherung in Abhängigkeit vom Ausmaß, in dem Kinderberücksichtigung schon in der Aktivzeit vorgenommen wird, variiert werden. ${ }^{213}$ (In der Altersphase müssen die ,eingesparten kinderbezogenen Renten' dann dem ,beitragsbezogenen Rententeil' zugeschlagen und entsprechend der dortigen Aufteilungslogik zugerechnet werden.)

Eine detaillierte Abschätzung der zukünftigen Beitragszahlungen eines Kindes schon in der Beitragsphase seiner Eltern (und insbesondere an deren Anfang) ist de facto unmöglich, so dass hier (bestenfalls) gestaffelte Pauschalierungen (z.B. nach Beitragsleistung und Qualifikation der Eltern) als Ansatz erscheinen, wenn auch mit sehr großen Unsicherheiten. Eventuell zu viel erhaltene ,Kinderberücksichtigungszahlungen' (z.B. wenn das Kind keine Beitragszahlungen zur Rentenversicherung leistet) wären dann in der Rentenphase den Eltern von ihren ,beitragsbezogenen Rentenansprüchen' (aufgezinst!) abzuziehen. Die bisher verdeutlichten Komplikationen eines derartigen Ansatzes lassen eine vorzeitige Kinderberücksichtigung im Rahmen der Rentenversicherung kaum implementierbar erscheinen. ${ }^{214}$

${ }^{212}$ Würde nur der Barwert der ,erwarteten Summe der Beitragszahlungen' des jeweiligen Kindes betrachtet, so würde damit der ,Versicherungscharakter' des Rentensystems vernachlässigt (Der Erwartungswert des Produktes zweier Zufallsvariablen entspricht nicht dem Produkt der Erwartungswerte der beiden Zufallsvariablen, wenn diese miteinander korreliert sind - und von einer solchen Korrelation ist auszugehen.).

${ }^{213}$ Die dadurch induzierte Änderung der Summe des Beitragsaufkommens ist übrigens nicht bei der Abschätzung des , angemessenen' Grades der Beitragsorientierung zu berïcksichtigen, da hier eine rein intragenerationale Umschichtung vorliegt. (Das höhere Beitragsaufkommen in Folge gesteigerter Kinderberucksichtigung schon in der Aktivzeit kommt nicht den ,Rentnern' zugute, sondern den Mitgliedern der Aktivgeneration.)

${ }^{214}$ Am ehesten möglich erscheint der Abkauf der spăteren kinderbezogenen Rentenansprüche durch eine Agentur. Da eine entsprechende Regelung aber auch fur ,beitragsbezogene Renten(teile)' möglich wäre, also auch im gegenwärtigen Rentensystem, von derartigen Märkten aber fast nichts bekannt ist (Bestenfalls könnte die Kreditvergabe an Leute, die erkennbar einen Teil ihrer späteren Rente zur Tilgung und Zinszahlung aufwenden müssen, so interpretiert 
Das gerade Gesagte verstärkt sich noch, wird keine , automatische' Kinderberücksichtigung während der Aktivzeit eingeführt, sondern ein ,Wahlrecht' auf vorzeitige Kinderberücksichtigung, und wird innerhalb der Rentenversicherung eine ,partielle Versicherung' gegen besonders geringe spätere Beitragszahlungen des Kindes zur GRV vorgenommen. ${ }^{215}$ In diesem Fall gewinnen asymmetrische Informationen (und u.U. auch moral hazard) an Bedeutung: Das Interesse der Eltern an einer Kinderberücksichtigung schon in der Aktivzeit ist (c.p.) umso höher, je geringer sie die zu erwartenden Beitragszahlungen des Kindes zur Rentenversicherung einschätzen. Ferner nimmt das Interesse zu, je mehr sich Versicherte als ,gute Risiken' für die Rentenversicherung sehen (also dann, wenn sie von einer kurzen Rentenbezugsdauer infolge frühen Todes ausgehen). Die letztere Eigenschaft kann - im Sinne einer Stärkung der ,Beitragsäquivalenz' der Rentenversicherung - begrüßt werden; doch auch dann ist zu beachten, dass die damit einhergehende Verringerung der Absicherung derjenigen, die das Alter erreichen, tendenziell eine Steigerung des allgemeinen Beitragsaufkommens erfordert, und somit nur schwerlich vermeidbar zu einer Mehrbelastung der nachfolgenden Generation führt.

Die erfolgten Ausführungen verdeutlichen, dass eine ,leistungsbezogene Kinderberücksichtigung' im Rahmen der Rentenversicherung kaum geeignet ist, eine Entlastung der Eltern schon während der Aktivzeit vorzunehmen, und eine Implementierung unvermeidlich mit sehr großen Problemen verbunden ist.

werden; doch hierbei wird regelmäßig vom Kreditgeber auf ein Kollateral z.B. in Form von anderweitigem Vermögen, insbesondere Immobilieneigentum, geachtet, so dass die Ruckzahlung über die etwaigen Rentenzahlungen hinaus gesichert ist.), sind die Marktversagensgründe wohl zu gravierend, um auf das Zustandekommen solcher Tauschmöglichkeiten zu setzen. Damit bliebe de facto wohl nur eine staatliche Ankaufsagentur ubrig. Die Probleme einer solchen Ankaufsregelung könnte aber auch diese kaum uberwinden.

${ }^{215}$ Z.B. in Form einer Begrenzung des Rückzahlungspflicht für zu hohe Kinderberücksichtigung schon in der Aktivzeit. 


\section{Kinderberücksichtigung im Rahmen einer zwangsweisen umlagefinanzierten Rentenversicherung - ein Vorschlag und die deutsche Realität}

Im vorangegangenen Kapitel wurden die distributiven Implikationen einer (unterlassenen) Kinderberücksichtigung im Rahmen einer umlagefinanzierten zwangsweisen Rentenversicherung (im Beitragssatzverfahren) untersucht. Hierbei wurde deutlich, welche gewaltigen Summen bei ,angemessener ${ }^{6}$ Kinderberücksichtigung nach anderen Kriterien als ihren Beitragszahlungen auf die Rentner aufzuteilen wären. Es ist dementsprechend nicht verwunderlich, dass sowohl in Vorschlägen zur Umgestaltung der bestehenden Ausgestaltung der Gesetzlichen Rentenversicherung, als auch in deren konkreten Ausgestaltung selbst Elemente der Kinderberücksichtigung enthalten sind. Dieses Kapitel befasst sich mit zwei solchen Ansätzen, und kontrastiert die ihnen immanente Kinderberücksichtigung mit den im vorangegangenen Kapitel gewonnenen Erkenntnissen.

\subsection{Der Vorschlag BORCHERTs zur Kinderberücksichtigung in der Rentenversicherung}

Zuerst soll auf den Vorschlag BORCHERTs zur Kinderberücksichtigung in der RV eingegangen werden, da er eine gewisse Bekanntheit und Popularität gewonnen hat.

\subsubsection{Darstellung des Vorschlags}

Ausgangspunkt BORCHERTs ist, dass „Kindererziehung und monetäre Beitragsleistungen .. beide für das Funktionieren der Gesetzlichen Rentenversicherung unersetzliche Voraussetzungen und somit konditional gleichwertig ${ }^{\text {(216 }}$ sind. Daraus folgert er die Notwendigkeit einer, Gleichbehandlung' der beiden Leistungen in Form einer „beitragsäquivalenten Berücksichtigung der Kindererziehung ${ }^{\text {“217. }}$. Die aus Beitragszahlungen resultierenden Rentenansprüche sollen - bei unverändertem Beitrag - gegenüber dem bisherigen Recht dementsprechend halbiert wer-

\footnotetext{
${ }^{216}$ BORCHERT (1981), S. 150 - Hervorhebungen im Original.

217 BORCHERT (1981), S. 163; vgl. auch BORCHERT (1993), S. 268.
} 
den. ${ }^{218}$ Für jedes Kind erhalten die Eltern zusätzlich eine Rente, die der Hälfte der durchschnittlichen Beitragsbemessungsgrenze zur RV multipliziert mit dem jeweiligen Beitragssatz zur GRV entspricht. Anders ausgedrückt: „[D]ie Hälfte der von einem Kind durchschnittlich eingezahlten Rentenbeiträge [fließt] auf das Rentenkonto der Eltern .., während die andere Hälfte zur Finanzierung des Barbeitragssystems verwandt wird“219. Die Verteilung des ,kinderbezogenen Rentenanspruchs' auf die beiden Elternteile erfolgt dann in umgekehrt proportionalem Verhältnis zu den ,beitragsbezogenen Rentenansprüchen' der Elternteile. Hat einer der Elternteile keine eigenen beitragsbezogenen Rentenansprüche, so erhält er die gesamte kinderbezogene Rente (für gemeinsame Kinder) des Ehepaars.

\subsubsection{Kritische Betrachtung des Vorschlags}

Die hälftige Aufteilung der Beitragseinnahmen auf ,beitrags-' und ,kinderbezogene' Renten bewirkt - gegenüber dem bisher bestehenden System - eine drastische Steigerung der ,Kinderberücksichtigung' (und somit spiegelbildlich eine entsprechend deutliche Senkung der ,Rendite' von Beitragszahlungen). Somit sind erhebliche Umstellungsprobleme hin zum neuen System zu erwarten; die Senkung der ,Rendite' der Beitragszahlungen mag Anreiz zur ,Flucht' in Schwarzarbeit etc. sein.

Die Abschätzung der Rentenhöhe im Rahmen des Vorschlags ist nicht möglich, da sich BORCHERT nicht auf eine exakte Methode der ,Kinderbewertung' festlegt. Zuerst schlägt er vor, den Eltern für jedes Kind die Hälfte des durchschnittlichen Rentenversicherungsbeitrags eines Versicherten ,auszuzahlen'. Da nicht bei allen Kindern die Eltern mehr leben würden, gebe es hierbei tendenziell ,überschüssige Mittel'. Ohne sich abschließend zu äußern, lässt BORCHERT eine Vorliebe dafür erkennen, die verbleibenden Mittel für eine Erhöhung der ,kinderbezogenen Renten' zu verwenden. ${ }^{220}$ Für diesen Fall errechnet GaLLON für das Jahr 1991 die in Tabelle 3 wiedergegebenen monatlichen Rentenwerte. ${ }^{221,222}$

${ }^{218}$ Vgl. für dieses und das Folgende: BORCHERT (1981), S. 229ff. und BORCHERT (1993), S. $270 \mathrm{ff}$.

219 BORCHERT (1981), S. 230.

${ }^{220}$ Vgl. BORCHERT (1981), S. 233f. Insoweit liegt eine Entsprechung zur ,Ausschöpfungskorrektur' im betrachteten Analyserahmen vor.

221

Vgl. GALLON (1993a), S. 12. 


\begin{tabular}{|c|c|c|c|}
\hline Rentenansprüche & Eltern zusammen & Mutter & Vater \\
\hline 1. aus Geldbeiträgen & \begin{tabular}{r|}
1.141 \\
\end{tabular} & 285 & 856 \\
\hline Verhältnis & \multicolumn{3}{|c|}{$1 \mathrm{zu} 3$} \\
\hline \multicolumn{4}{|l|}{ Plus } \\
\hline \multicolumn{4}{|l|}{ 2. wegen Erziehung } \\
\hline Verhältnis & \multicolumn{3}{|c|}{$3 \mathrm{zu} 1$} \\
\hline $1 \mathrm{Kind}$ & 935 & 701 & 234 \\
\hline 2 Kinder & 1.870 & 1.403 & 467 \\
\hline 3 Kinder & 2.805 & 2.104 & 701 \\
\hline 4 Kinder & 3.740 & 2.805 & 935 \\
\hline
\end{tabular}

\section{Tabelle 3: Berechnungen GaLlons zu den Rentenwirkungen des BORCHERT-Vorschlags}

Anmerkung: Der kinderbezogene Rentenwert von DM 935 pro Kind errechnet sich aus dem Produkt der Hälfte der durchschnittlichen Beitragszahlung mit dem im Jahre 1991 bestehenden ,Eckrentnerverhältnis' (Zahl der Beitragszahler pro Bezieher einer ,Standardrente'), das GALLON (1993a, S. 12) mit ca. 2,85 ansetzt.

BORCHERT rechtfertigt die Höhe der kinderbezogenen Renten mit den hohen Kosten der Kindererziehung. ${ }^{223,224}$ Die Argumentation erscheint allerdings nicht unpro-

${ }^{222}$ Zum Vergleich mit der Analyse aus der vorliegenden Arbeit seien kurz die ,kumulierten Rentenwerte' eines Kindes mit durchschnittlichem Einkommen für $\alpha=U, \supset$ (für das Jahr 1994) angegeben: korrigiert (ca.) DM 935 / Monat, unkorrigiert (ca.) DM 719 / Monat (vgl. Tabelle 2). Bei Umrechnung auf das Jahr 1991 ergeben sich (unter Nutzung der Steigerungssätze für laufende Renten in Westdeutschland) Werte von (ca.) DM 835 (641) / Monat (statt DM 935 resp. 719).

BORCHERT (1981), S. 231: „Es stellt sich ... die Frage, ob die Halbierung der monetär bestimmten Rentenleistungen und die Verwendung der dadurch freigewordenen Mittel für das Elternrentensystem nicht die Erziehung überbewertet; wăhrend nămlich für den Lohnersatzanspruch ein Arbeitsleben von durchschnittlich 40 Jahren erfullt sein muß, reichen bei der Elternrente 18 Jahre Erziehung aus.

Die Bewertung ist jedoch gerecht. Sie berücksichtigt außer der Betreuung nämlich auch die materiellen Unterhaltsaufwendungen für das Kind, die im Jahre 1978 im Monat durchschnittlich pro Kind 600,- DM betrugen. Diese Summe als Barbeitrag zur Rentenversicherung geleistet, würde einem versicherten Einkommen von 3.333,- DM entsprechen. ... [D]ieser Beitrag [kommt] .. etwa dem 1,8fachen des damals versicherten Druchschnittseinkommens [sic!] gleich.“ Es sei zu BORCHERTs Argumentation angemerkt, dass hier eine demographisch bedingte Erhöhung des Beitragssatzes zur GRV (z.B. wegen steigendem Alterslastquotienten) eine verringerte Berücksichtigung von Kindern im Rahmen der RV rechtfertigt, da die Kinderkosten jetzt ja einem geringeren ,Einkommensăquivalent' entsprechen. Die Kosten des Kinder- 
blematisch: So ist zum einen zu hinterfragen, ob die RV der richtige Ort zur Berücksichtigung von Kosten der Kindererziehung ist - und nicht das allgemeine Steuersystem. ${ }^{225}$ Kosten der Kindererziehung per se generieren keine (späteren) Renten(beitragszahlungen). BORCHERT setzt mit seiner Begründung gerade an einem Punkt an, der für die spätere Funktionsfähigkeit der RV nicht unmittelbar erheblich ist - wesentlich ist nicht der Input (Kosten der Kindererziehung), sondern der Output (Beitragszahlungen der Kinder in ihrer Aktivzeit). Nun einmal unterstellt, die GRV sei ein systematisch ,richtiger' Ort zur Berücksichtigung von Kosten der Kindererziehung: Dann stellt sich unmittelbar die Frage, warum der Familie von Seiten der RV nicht geholfen wird, wenn der dahingehende Bedarf am größten ist, nämlich in der Zeit der Kindererziehung. Stattdessen kommt es zu erhöhten Renten in der Zeit, in der die Kosten nicht anfallen. Gerade bei ,kostenseitiger' Begründung einer Kinderberücksichtigung in der GRV wäre eine Synchronisation von Bedarf und der Zahlung einer kinderbezogenen Leistung der Rentenversicherung besonders angemessen. ${ }^{226}$ Dieser Punkt ist systematisch von Bedeutung, da BORCHERT die Rentenzahlung nicht von - erst später zu beobachtenden - Charakteristika des Kindes (Beitragszahlungen zur GRV etc.) explizit abhängig macht. Folglich wäre grundsätzlich eine Beitragsentlastung der Kindeseltern in der ,Erziehungsphase' theoretisch möglich (z.B. pro Kind um den Betrag, der dem Produkt des hälftigen Beitragssatzes mit dem durchschnittlichen rentenversicherungspflichtigen Einkommen entspricht) ${ }^{227}$.

Die Problematik einer ,kostenorientierten' Begründung einer Kinderberücksichtigung bei der Rente wird besonders deutlich, wird ein seinen Lebensunterhalt

habens sind jedoch in der Vergangenheit angefallen - und dementsprechend nicht (bzw. nur äußerst indirekt) vom generativen Verhalten der Generation abhängig.

224 Opportunitätskosten erwähnt BORCHERT begrifflich nicht - er verweist aber z.B. auf die Unterbrechung der Erwerbstätigkeit auf Seiten der Frau zugunsten der Kindererziehung.

${ }^{225}$ Eine Berücksichtigung der ,Kosten' der Kindererziehung im Rahmen der Rentenversicherung wäre anders zu bewerten, wenn sie nicht Ziel, sondern Mittel wäre: In diesem Fall könnte eine Kostenorientierung mit der Begrundung einer positiven Korrelation der (typisierten) Kosten der Kindererziehung mit dem späteren (durchschnittlichen), Wert' dieser Kinder für die Rentenversicherung erfolgen. Eine solche Begrundung lässt sich bei BORCHERT aber nicht finden.

${ }^{226}$ Auch in einer späteren Stellungnahme sieht BORCHERT nicht, dass zur Behebung von (etwaiger) Familienarmut die von ihm vorgeschlagenen Änderungen in der RV nur äußerst eingeschränkt tauglich sind (vgl. BORCHERT (1994)).

${ }^{227}$ Wobei das Problem der Finanzierung einer solchen Ausgestaltung in der Anfangsphase nach ihrer Einführung besteht - hier entstehen Ausgaben, die erst in einer späteren Phase (Rentenphase) durch ,Entlastungen' infolge niedrigerer Rentenzahlbeträge ausgeglichen werden. 
dauerhaft von ,Sozialhilfe-Zahlungen' bestreitendes Ehepaar mit nennenswerter Kinderzahl betrachtet: Die gesamten monetären Kosten der Kindererziehung werden von der Gemeinschaft der Steuerzahler finanziert. Mit der von BORCHERT vorgetragenen Begründung lässt sich in diesem Fall nicht erklären, warum ein Paar, das de facto nur Geld anderer Leute für seine Kinder verausgabt hat, ${ }^{228}$ im Alter eine kinderbezogene Rente in derselben Höhe erhalten soll wie ein Paar, das die Erziehung der eigenen Kinder in erheblichem Maße eigenfinanziert hat. Zwar lässt sich eine rentenmäßige Berücksichtigung der Kinder auch von Sozialhilfeempfängern mit Hilfe von Opportunitätskostenerwägungen rechtfertigen - die übereinstimmende Höhe der rentenmäßigen Kinderberücksichtigung unabhängig von der Aufbringung der Finanzmittel bedarf dann aber einer gesonderten resp. anderen Begründung. ${ }^{229}$

Nach diesen ,grundsätzlichen' Erörterungen ist noch auf ein ,technisches' Problem zu verweisen: Das Verfahren ist nicht sicher vor der ,Überausschöpfung' der Finanzmittel (es werden Rentenzusagen gegeben, die u.U. nicht einlösbar sind auch nicht bei einer Erhöhung der Beiträge!). Ist nur die Hälfte der Kinder einer Generation rentenversicherungspflichtig erwerbstätig (z.B. weil sich der andere Teil um die Kindererziehung kümmert; aber auch bei anderweitiger ,Heimproduktion', ,Aussteigertum', Arbeitslosigkeit, Selbstständigkeit oder Berufsbeamtentum), so stehen u.U. jedem erwerbstätigen Kind zwei Elternpaare gegenüber (z.B. Eltern und Schwiegereltern), die jeweils die Hälfte eines durchschnittlichen Beitrags erhalten - für beitragsbezogene Renten bleibt in diesem Fall kein Platz mehr (und eine Erhöhung des Beitragssatzes bietet keinen ,Ausweg', da hierbei die kinderbezogenen Rentenansprüche entsprechend steigen). In der Praxis ist dieses Problem (natürlich) vollkommen unbedeutend, da ein nicht unerheblicher Teil der Eltern der Aktivgeneration entweder noch keine Rente bezieht (da zu jung) oder aber verstorben ist, und mehr als die Hälfte der Mitglieder der Aktivgeneration einer rentenversicherungspflichtigen Erwerbstätigkeit nachkommen doch als grundsätzliches Problem bleibt das oben beschriebene Konstrukt beste-

${ }^{228}$ Noch dazu liegen nur geringe Opportunitätskosten des Kinderhabens in Form von Verdienstausfall vor, so dass sich auch hier kein Begründungsansatz für eine hohe Erziehungsrente ergibt!

${ }^{229}$ In dem Ansatz BORCHERTs lohnt es sich für Familien mit sehr niedrigem (potenziellen) Erwerbseinkommen, auf die Ausübung einer Erwerbstätigkeit zu verzichten und sich auf die ,Kinderaufzucht' zu spezialisieren. Der dabei zu erzielende Lebensstandard im Alter ist nicht anders zu realisieren - und der eigene finanzielle Einsatz (aber auch die Opportunitătskosten in Form von Verdienstausfall, entgangenen Freizeitaktivităten etc.) vergleichsweise gering. Zwar sind die Vorteile einer arbeitsteiligen Produktion bekannt (und unbestritten), ob dies auch fur die gerade vorgestellte Form der Arbeitsteilung gilt, kann hier nicht entschieden werden. 
hen. ${ }^{230}$ Und die Anreizwirkungen des Ausgestaltungsvorschlags gehen hin zu einer Verringerung der Erwerbstätigkeit auf Seiten eines Elternteils. ${ }^{231,232}$

Abschließend sei auf das bereits an anderer Stelle (vgl. 4.3.3.1) thematisierte Problem der ,hälftigen Aufteilung' der Beitragseinnahmen auf beitrags- und kinderinduzierte Rentenansprüche verwiesen - eine grundsätzliche Diskussion resp. Rechtfertigung dieser Halbteilung erfolgt nicht. ${ }^{233}$

\subsubsection{Vergleich mit der vorliegenden Untersuchung}

Sowohl der Vorschlag BORCHERTs als auch der in dieser Arbeit vorgestellte Analyserahmen basieren auf einer grundsätzlichen rentenmäßigen Anerkennung des eigenständigen Wertes der Heranziehung einer neuen Generation zukünftiger Mitglieder der GRV. Sie unterscheiden sich damit fundamental von der gegenwärtigen Ausgestaltung, die die Bedeutung der zweiten Säule neben der Beitragszahlung, nämlich der Kindererziehung, weitgehend ignoriert.

Der systematisch bedeutsamste Unterschied besteht in der Methode der Kinderberücksichtigung. BORCHERT erhöht den Rentenanspruch für jedes Kind um ein und denselben Betrag, unabhängig von seinen versicherungsrelevanten Eigenschaften. Kinder mit hohen Beitragszahlungen werden mithin ,unter-', Kinder mit niedrigen Beitragszahlungen hingegen ,überbewertet'. BORCHERT akzeptiert kinderbezogene Rentenzahlungen auch für Kinder, die nie in die GRV eintreten. Die Analyse im Rahmen der vorliegenden Arbeit legt hingegen nahe, die kinderbezogenen Rentenansprüche in ihrer Höhe (positiv) abhängig von den Beitragszahlungen der Kinder auszugestalten, da höhere Beitragszahlungen positive externe Effekte für

${ }^{230}$ Den heute ca. 22,14 Millionen sozialversicherungspflichtig Beschäftigten stehen ca. 16,4 Mill. Bezieher von Versichertenrenten gegenüber (von denen allerdings ,nur' 9,7 Mill. Regelaltersrente beziehen!).

${ }^{231} \mathrm{Zu}$ Letzterem vgl. GALLON (1993a), S. 11.

${ }^{232}$ Das geschilderte Problem existiert nicht, wird auf das durchschnittliche rentenversicherungspflichtige Einkommen aller Kinder abgezielt, wobei Kinder, die keine Beitragszahlungen zur GRV leisten, mit einem Einkommen von 0 in die Durchschnittsbildung eingehen. Dies entspräche aber nicht der bisherigen Vorgehensweise bei der Ermittlung des entsprechenden Wertes.

${ }^{233}$ Auch in den positiven Rezensionen des Vorschlags BORCHERTs durch OETER (1989a, 1989b) fehlt jeder Hinweis auf eine weitergehende Begründung der Halbteilung des Beitragsaufkommens. 
die Rentnergeneration induzieren. Bei einer solchen Ausgestaltung wird die elterliche Investition in ,Kinder-Qualität' gegenüber einer Investition in ,KinderQuantität' nicht diskriminiert.

Die Unterschiede in der Ausgestaltung der Kinderberücksichtigung sind (u.a.) Folge unterschiedlicher Zielvorstellungen. BORCHERT betont die Kostenseite des Kinderhabens, die positiven externen Effekte hingegen betrachtet er nur äußerst pauschal. Die kinderbezogenen Renten dienen bei ihm zum (teilweisen) Ausgleich des kinderbedingten Kosten der Eltern. ${ }^{234}$ Für die vorliegende Untersuchung hat die Kinderberücksichtigung in der RV dagegen nicht die Aufgabe, einen Ausgleich für die den Eltern durch das Kinderhaben entstandenen Kosten zu schaffen, sondern vielmehr die für die Gesellschaft erbrachten Leistungen rentenwirksam zu berücksichtigen. Da BORCHERT seine Untersuchung als Vorschlag zur Ausgestaltung der Rentenversicherung versteht, muss er Praktikabilitätsfragen berücksichtigen. Dies ist bei einer reinen Analyse, die keinen Anspruch erhebt, aus ihr lasse sich unmittelbar eine in der Praxis implementierbare Ausgestaltung der Rentenversicherung ableiten, nicht der Fall. Auch hieraus lassen sich Unterschiede zwischen den Ansätzen erklären.

Es ist unvermeidlich, dass eine Zwangsmitgliedschaft in einer umlagefinanzierten RV immer zu besonderen Problemen bezüglich der ,richtigen' Berücksichtigung der Kindererziehung führt. Eine in Teilen kritisierbare Ausgestaltung der ,Kinderberücksichtigung' ist de facto wohl unumgänglich. Die geschilderte Indeterminiertheit sollte aber nicht dazu verleiten, auf jedwede Kinderberücksichtigung (im über die ,reine Hinterbliebenenabsicherung' hinausgehenden Teil) zu verzichten. Unsicherheit kann über die Höhe und die genaue Ausgestaltung einer ,richtigen' Anerkennung von Erziehungsleistungen im umlagefinanzierten Rentenversicherungssystem bestehen, nicht aber in der Tatsache, dass Eltern mit Kindern, die ihrerseits die RV fortführen, einen Beitrag zum Erhalt der RV leisten, dessen NichtAnerkennung aus allokativen und distributiven Gründen falsch wäre.

234 In einer späteren Darstellung schlägt BORCHERT (1993, S. 270f.) vor, zur Vermeidung von ,Überversorgungen' die Zahl der rentenrechtlich anzuerkennenden Kindern auf (maximal) 4 zu beschränken - mit der Begrundung, analog sei auch eine Beschränkung der ,beitragsinduzierten' Rente gegeben. Diese Argumentation ist erstaunlich, bedenkt man, dass er die kinderbezogene Rente mit den Kosten der Kindeserziehung begründet. Auch der Verweis auf eine Obergrenze beitragsbezogener Renten trägt nicht, denn im beitragsbezogenen Teil kommt es parallel zur Kappung der beitragsbezogenen Anspruche auch zu einer Begrenzung der Höhe des rentenversicherungspflichtigen Einkommens, d.h. der monatlichen Beitragszahlungen bzw. der Kosten der Alterssicherung. Ein entsprechender Mechanismus der vollständigen ,Kostenentlastung' existiert bei der Kindererziehung nicht. 


\subsection{Die gegenwärtige Regelung im deutschen Rentenrecht in kritischer Sicht}

Im Folgenden soll auf die im gegenwärtigen (deutschen) Rentenrecht existierende Kinderberücksichtigung eingegangen werden. ${ }^{235}$ Hierzu wird zuerst die Regelung der Kinderberücksichtigung bei der Altersrente (skizzenhaft) dargestellt, um sie dann nach Struktur und Umfang untergliedert zu bewerten. Daran anschließend werden weitere Elemente der Kinderberücksichtigung im deutschen Rentenrecht kurz angesprochen. ${ }^{236}$ Ferner wird thematisiert, inwieweit der erst eingeführte und dann noch vor Inkrafttreten (von einer veränderten Bundestagsmehrheit) zurückgestellte ,demographische Faktor' ein Element der Kinderberücksichtigung darstellt.

Der vorliegende Teil der Arbeit ist der einzige, der andere Funktionen der Rentenversicherung als die der Absicherung eines Alterseinkommens systematisch in die Erörterungen einbezieht.

\subsubsection{Die derzeitige Kinderberücksichtigung bei der Altersrente}

\subsubsection{Darstellung der Kinderberücksichtigung bei der Altersrente}

Grundsätzlich wird die Erziehung von Kindern im Rahmen der GRV rentenerhöhend berücksichtigt. $\S 3$ des Sechsten Sozialgesetzbuches (SGB) legt fest, dass "Personen in der Zeit, .. für die ihnen Kindererziehungszeiten anzurechnen sind" „[v]ersicherungspflichtig sind”. Die genauere Regelung für die Behandlung von Kindererziehungszeiten findet sich in $\S 56 \mathrm{Absatz} 1 \mathrm{Satz} 1$. Hier wird festgelegt, dass „für Zeiten der Erziehung eines Kindes in dessen ersten drei Lebensjahren .. Pflichtbeiträge" als gezahlt gelten. Grundsätzlich wird die Erziehungszeit einem Elternteil angerechnet, doch ist eine zeitliche Aufteilung auf beide Elternteile möglich. ${ }^{237}$ Der gesetzlich vorgesehene Normalfall ist eine Erziehung durch die

${ }^{235}$ Schon zu Beginn der Ausführungen sei darauf hingewiesen, dass es hierbei nur um eine rudimentäre Darstellung des Systems ohne Behandlung einzelner Sonderfälle geht. Insbesondere wird darauf verzichtet, auf Besonderheiten der knappschaftlichen Alterssicherung oder auf die Regelungen des Versorgungsausgleichs bei Scheidung einzugehen. Auch unterbleibt ein Verweis auf gesonderte (Übergangs-)Regelungen für das Beitrittsgebiet.

${ }^{236}$ Für eine (skizzenhafte) Darstellung der rentenrechtlichen Regelungen zur Kinderberücksichtigung in anderen europäischen Ländern sei auf HOHNERLEIN (1992) verwiesen.

${ }^{237}$ Eine solche Aufteilung erfordert eine Erklärung beider Elternteile und kann nur für die zukünftige Erziehungszeit sowie rückwärtig für zwei Kalendermonate (soweit dem nicht andere 172 
Mutter: „Haben die Eltern eine übereinstimmende Erklärung [darüber, wer das Kind erzieht; d. Verf.] nicht abgegeben, ist die Erziehungszeit der Mutter zuzuordnen" ${ }^{238}$. „Die Kindererziehungszeit beginnt nach Ablauf des Monats der Geburt und endet nach 36 Kalendermonaten"239, wobei sich diese Frist bei der Geburt weiterer Kinder während einer Kindererziehungszeit um 36 Monate je weiterem Kind verlängert. ${ }^{240}$ Die Tatsache, dass für die Kindererziehung Beiträge als gezahlt gelten, sagt per se natürlich noch nichts über die konkrete Höhe der als gezahlt geltenden Beiträge, und damit über deren Auswirkungen auf die Höhe der (späteren) Rente der Erziehenden, aus. An dieser Stelle hat es in der letzten Zeit die bedeutendsten Änderungen - sowohl systematischer Natur, als auch die Höhe der Kinderberücksichtigung betreffend - gegeben. Bis Mitte 1998 galt, dass für die ersten drei Jahre nach der Geburt eines Kindes jeweils 0,75 persönliche Entgeltpunkte beitragsfrei angerechnet wurden. Hierbei ist zu berücksichtigen, dass bei einer Regelaltersrente -1 persönlicher Entgeltpunkt zur Zeit (ab dem 1.Juli 1999) zu einer Erhöhung des monatlichen Rentenbetrags um DM 48,29 führt. ${ }^{241}$ 0,75 persönliche Entgeltpunkte erhöhten den monatlichen Rentenbetrag mithin um DM 36,22 und 2,25 Punkte (maximale ,Gesamtpunktzahl' pro Kind) entsprechend um DM 108,66 / Monat. ${ }^{242}$ Allerdings wurden die kinderbezogenen persönlichen Entgeltpunkte nicht automatisch zugerechnet. Vielmehr erfolgte eine $\mathrm{Zu}$ rechnung in den ersten drei Jahren nach Geburt des Kindes nur insoweit, wie in dieser Zeit nicht durch Beitragszahlungen eigenständig Entgeltpunkte erzielt wurden. Mithin wurden eventuelle Beitragszahlungen in dieser Zeit vollständig angerechnet. ${ }^{243}$ Eine zeitliche Verschiebung der ,Kindererziehungszeit' war grundsätzlich nicht möglich - einzige Ausnahme war die schon erwähnte Verlängerung des

Regelungen, auf die hier nicht năher eingegangen werde, entgegenstehen - vgl. SGB VI $\S 56(4)$ ) bewirkt werden (vgl. SGB VI $§ 56(2)$ ).

${ }^{238}$ SGB VI $\S 56(2)^{8}$. Ferner gilt: „Haben mehrere Elternteile das Kind erzogen, ist die Erziehungszeit demjenigen zuzuordnen, der das Kind uberwiegend erzogen hat" (SGB VI $\left.\S 56(2)^{9}\right)$.

${ }^{239}$ SGB VI $\S 56(5)^{1}$. ,Kindererziehungszeit' meint hierbei den rechtlich bestimmten Begriff und nicht die faktische Länge der Kindererziehung.

${ }^{240}$ SGB VI $\S 56(5)^{2}$.

${ }^{241}$ Aus Gründen der Vergleichbarkeit wird auf den gegenwärtigen , aktuellen Rentenwert' Bezug genommen.

${ }^{242}$ Geringfügige Abweichungen durch Rundungen!

${ }^{243}$ SCHMIDT und GASCH (1993, insbes. S. 263) werfen dieser Regelung im Rentenrecht vor, einseitig an einer unterstellten Unvereinbarkeit von Kindererziehung und Berufstätigkeit beider Ehepartner ausgerichtet zu sein. 
Zeitraumes um weitere drei Jahre bei der Geburt eines weiteren Kindes innerhalb der Drei-Jahres-Periode.

Unter dem Druck des Bundesverfassungsgerichts kam es zum 01. Juli $1998 \mathrm{zu}$ einer systematisch bedeutsamen Änderung des Rentenrechts: Zu diesem Zeitpunkt wurde einerseits die rentenmäßige Bewertung von Kindererziehungszeiten auf 1,0 Entgeltpunkt / Jahr erhöht, andererseits werden die Entgeltpunkte jetzt additiv den Entgeltpunkten des betreffenden Zeitraums zugeschlagen - es erfolgt also keine Anrechnung auf durch im entsprechenden Zeitraum mit Beitragsleistungen erworbene Entgeltpunkte. Allerdings ist zu beachten, dass die Additivität nur gilt, soweit nicht die für die jeweiligen Kalenderjahre festgelegten Höchstwerte der erzielbaren Entgeltpunkte erreicht werden. ${ }^{244}$ Eine ,kinderbedingte' Überschreitung dieser Höchstwerte ist nicht möglich - auch dann nicht, wenn in vorangegangenen oder nachfolgenden Jahren die Höchstwerte von dem Versicherten nicht erreicht worden sind. Für bereits bestehende Renten(ansprüche) wird die ,Höherbewertung' der Kindererziehungszeiten zeitlich gestaffelt: Vom 01.07.1998 bis zum 30.06.1999 werden pro Kind 0,85, vom 01.07.1999 bis zum 30.06.2000 0,9 Entgeltpunkte angerechnet. ${ }^{245}$ Erst danach erfolgt die Rentenberechnung mit dem Entgeltpunktwert von 1,0 .

Die gerade dargestellte Regelung gilt allerdings erst für Eltern ab dem 01. Januar 1992 geborener Kinder! Bei vor diesem Datum geborenen Kindern beträgt die (rentenwirksame) Kindererziehungszeit 12 Monate, ${ }^{246}$ wobei der Elternteil, dem die Erziehungszeit angerechnet werden soll, nicht vor dem 01. Januar 1921 geboren sein darf. ${ }^{247}$ Für vor diesem Datum geborene Eltern wurde in $\S 294$ des Sechsten Sozialgesetzbuches die sukzessive Einführung einer ,Leistung für Kindererziehung' veranlasst, die in ihrer Höhe dem Wert vor 1992 geborener Kinder entspricht. ${ }^{248}$

${ }^{244}$ Vgl. SGB VI $§ 70$ (2) (mit Bezug auf SGB VI Anlage 2b). Der, jăhrliche Höchstwert an Entgeltpunkten' ergibt sich als Quotient der ,jährlichen Beitragsbemessungsgrenze' und des ,Durchschnittsentgelts' des jeweiligen Jahres (mit Rundung auf vier Nachkommastellen). Der Wert schwankt im Zeitablauf; seit 1983 liegt er regelmäßig im Bereich von ca. 1,80 - 1,85.

Vgl. SGB VI § 307d.

246 Vgl. SGB VI § 249 (1).

247 Vgl. SGB VI § 249 (4).

${ }^{248}$ Die Einführung ist inzwischen abgeschlossen. 
Ein - heute geborenes - Kind erhöht die Zahl der Entgeltpunkte seiner Eltern also um (im Normalfall) 3,0, was bei einem aktuellem Rentenwert von DM 48,29 in den alten Bundesländern eine Rentensteigerung um DM 144,87 / Monat bedeutet.

Die Finanzierung der rentenmäßigen Berücksichtigung von Kindererziehungszeiten erfolgt seit 1999 durch „,echte Beiträge des Bundes für die Kindererziehung (zusätzliche Leistungsansprüche entstehen dadurch nicht) ${ }^{‘ 249}-\S 177$ SGB VI lautet nun: „Die Beiträge für Kindererziehungszeiten werden vom Bund getragen". 1992 lagen die Erstattungen des Bundes für die Kindererziehungszeiten in der GRV pauschal bei DM 4,8 Mrd. / Jahr, und wurden in den darauffolgenden Jahren mit der allgemeinen Steigerungsrate des Bundeszuschusses bis auf DM 7,2 Mrd. im Jahre 1998 erhöht. ${ }^{250}$ Diese Zahlung wird in den Jahren 1999 und 2000 auf null abgesenkt. Für die Jahre 1999 und 2000 erfolgt eine pauschalierende Zahlung, die im Jahre 1999 (für die Zeit ab dem 01. Juni) DM 13,6 Mrd. und im Jahre 2000 DM 22,4 Mrd. beträgt. ${ }^{251}$ In den nachfolgenden Jahren entspricht die Zahlung dann jeweils der Summe, die sich bei Absicherung eines Elternteils jedes bis zu 3 Jahre alten Kindes mittels Beitragszahlung zur Rentenversicherung ergibt. ${ }^{252}$ Die Mehrbelastung des Bundes aus der geschilderten Reform der Kinderberücksichtigung wird für 1999 mit ca. DM 12,1 Mrd. angegeben. ${ }^{253}$

\subsubsection{Bewertung der Ausgestaltung}

Man kann zweifelsfrei festhalten, dass die Bedeutung von Kindern für die Festlegung der jeweiligen Rentenhöhe von Versicherten in den letzten Jahren erheblich gesteigert worden ist. Es ist bei einer solchen Bewertung aber zu beachten, dass diese Steigerung quasi von einem ,Null-Niveau' aus begann, was zu hohen Steigerungsraten schon bei vergleichsweise geringen absoluten Änderungen führt.

Im Sinne der vorangegangenen grundsätzlichen Erörterungen der Sinnhaftigkeit einer Kinderberücksichtigung im Rahmen einer im Umlageverfahren organisier-

${ }^{249}$ DEUTSCHER BUNDESTAG Drucksache 14 / 45 vom 17.11.1998, Gesetzentwurf der Fraktionen SPD und BÜNDNIS 90 / DIE GRÜNEN.

${ }^{250}$ Vgl. DeUTSCHER BundeSTAG Drucksache 14/45 vom 17.11.1998. Der Zuschuss deckte im Jahr 1998 ungefähr die Zahlungsverpflichtungen der GRV für Kindererziehungszeiten ab, die 1995 ca. DM 6,4 Milliarden betrugen.

${ }^{251}$ Vgl. SGB VI $§ 279 f$.

${ }^{252}$ Vgl. SGB VI $§ 279 f(1)^{2}$.

${ }^{253}$ Vgl. DeUTSCHER BundeSTAG Drucksache 14 / 45 vom 17.11.1998. 
ten Zwangs-Rentenversicherung erscheint die aufgezeigte Tendenz einer stärkeren rentensteigernden Berücksichtigung von Kindern begrüßenswert. Zu hinterfragen bleiben aber Ausmaß und konkrete Ausgestaltung dieser Kinderberücksichtigung.

Die sukzessive Einführung gerade für alte Mütter (vor 1921 Geborene) sowie die unterschiedliche Höhe der monatlichen Kinderberücksichtigung in Abhängigkeit vom Geburtsdatum der Mutter hatten das Ziel, die Kosten der Einführung kurzfristig vergleichsweise niedrig zu halten. Dieses ist per se auch nicht kritikwürdig; ob das gewählte Verfahren, das ein Versterben vieler Mütter vor erstmaligem Anspruch auf die kinderbezogene Rente zur Folge hatte, ein angemessener Weg der Kostenreduktion war, kann hier nicht geklärt werden. ${ }^{254}$

Die Unterscheidung der Höhe des rentenmäßigen Wertes von Kindern nach dem Geburtsdatum ist aus theoretischer Sicht interessant: Logik der Ausgestaltung ist es, das zukünftige Kinderhaben zu fördern bzw. grundsätzlich als eigenständige Leistung zum Erhalt der Rentenversicherung anzuerkennen. Wenn das Ziel der Implementierung der rentenmäßigen Kinderberücksichtigung darin besteht, ein weiteres Absinken der Geburtenrate zu verhindern (bzw. eine Steigerung zu erreichen), so ist es von daher konsequent, nur nach Einführung der Kinderberücksichtigung geborene Kinder rentensteigernd zu berücksichtigen. ${ }^{255}$ Außerdem mag eine unterschiedliche rentenmäßige Berücksichtigung von Kindern in Abhängigkeit vom Geburtsdatum der Mutter noch anderweitig begründet werden: Aus allokativen und distributiven Gründen ist eine Berücksichtigung der Kinderzahl um so eher geboten, je größer die relativen Unterschiede zwischen den Kinderzahlen der Mitglieder einer Rentnergeneration (bzw. den potenziell Rentenberechtigten) sind. Insoweit können intergenerational zunehmende Unterschiede zwischen den Kinderzahlen der Mitglieder einer Generation eine Begründung für eine sukzessive (und nach der Alterskohorte der Eltern gestaffelte) Einführung einer Kinderberücksichtigung liefern.

${ }^{254}$ Das Bundesverfassungsgericht hat in einer Entscheidung vom 07.07.1992 (BVerfGE, Vol. 87 (1992), Nr. 1 v. 07.07.1992) die sukzessive Einfuhrung einer rentenmäßigen Anrechnung von Kindererziehungszeiten für verfassungskonform befunden. Vgl. Anhang 5 (Abschnitt 7.5).

${ }^{255}$ Man könnte sogar noch weiter gehen, und die in den ersten Monaten nach Einführung der Regelung geborenen Kinder ebenfalls nicht rentenwirksam werden lassen - immerhin ist die Entscheidung zugunsten dieser Kinder auf Seiten der Eltern bereits gefallen, so dass die Einfuhrung der Regelung keine unmittelbare Wirkung auf die Fertilităt hat. Das gerade Gesagte ist aber einerseits insoweit zu revidieren, als bereits vor Verabschiedung des Gesetzes eine Erwartung bezüglich der Implementierung existiert haben dürfte, andererseits ist ein allgemeiner (positiver) Einkommenseffekt einer erwarteten höheren Rente (infolge der Kinderberlicksichtigung) möglicherweise die Wahrscheinlichkeit der Geburt eines weiteren Kindes steigernd. 
Dass die faktische Ausgestaltung der Einführung der Kinderberücksichtigung im deutschen Rentenrecht derart zu begründen ist, ist allerdings anzuzweifeln: Die gewählten Unterschiede im Alter der Mütter sind zu gering, um als Proxy-Variablen für unterschiedliche durchschnittliche Kinderzahlen der Mitglieder der jeweiligen Generation zu dienen. Bedeutsamer ist jedoch eine grundsätzliche Feststellung: Die nachträgliche rentenwirksame Berücksichtigung bereits zum Zeitpunkt der Einführung der Regelung geborener Kinder ist wohl nicht mehr mit ,pro-natalistischen' Erwägungen zu begründen. ${ }^{256} \mathrm{Sie}$ ist vielmehr ein Indiz dafür, dass der Gesetzgeber die Bedeutung von Kindern für den Erhalt der Rentenversicherung grundsätzlich anerkennt.

Die gewählte Form der Kinderberücksichtigung ist aus verschiedenen Gründen problematisch: Zum einen besteht durch die gewählte Ausgestaltung die Tendenz zu einer Lastverschiebung hin zur Kindergeneration. ${ }^{257}$ Die Gewährung von Erziehungszeiten ist kurzfristig nur geringfügig kassenwirksam, erhöht aber den Bestand an Forderungen gegenüber dem Rentenversicherungssystem, induziert folglich spätere Zahlungsverpflichtungen. ${ }^{258}$ Dies wäre weniger problematisch, wäre den Versicherten bekannt, dass der absolute Wert ihrer Rentenansprüche vom absoluten Forderungsbestand aller Rentenbezugsberechtigten abhängt, so dass eine Ausweitung von Ansprüchen c.p. zur Minderung des einzelnen Anspruchs führt. Diese Senkung des einzelnen Rentenanspruchs kann durch spätere staatliche Zuschüsse zur Rentenversicherung sowie Erhöhungen des Beitragssatzes vermieden bzw. verringert werden. In beiden Fällen - unabhängig von einer

${ }^{256}$ Bestenfalls kann gesagt werden, die rückwirkende Gewăhrung von Kinderrenten erhöhe die Glaubwürdigkeit einer entsprechenden Zusage für in Zukunft geborene Kinder. In Anbetracht der Starre des deutschen Sozialsystems, die die Abschaffung einmal eingeführter Leistungstatbestände de facto unmöglich macht, und des weitgehenden politischen Konsenses bezüglich der Einführung einer Kinderberücksichtigung, erscheint eine derartige Begründung der rückwirkenden Rentengewăhrung aber nicht sonderlich überzeugend. Die (,rückwirkende') Berücksichtigung stellt allerdings eine Investition in Sozialkapital dar - vorausgesetzt, sie ist distributiv geboten!

${ }^{257}$ Vgl. z.B. DINKEL (1981), S. 139, LÜDEKE (1995), S. 175. Vgl. in diesem Zusammenhang auch HACKMANN (1990).

${ }^{258}$ Seit dem Jahr 1999 zahlt der Bund fur die Anrechnung von Kindererziehungszeiten parallel zur Entstehung des Rentenanspruchs Beiträge. Da die Mittel nicht in dem Augenblick zur Verfugung gestellt werden, in dem die betroffenen Eltern Rente beziehen, oder zum Aufbau einer Kapitalfundierung der späteren kinderbezogenen Rentenansprüche genutzt werden, führen sie zu einer kurzfristigen Verbesserung der Finanzsituation der GRV, ohne die späteren Möglichkeiten der GRV zur Leistung der kinderbezogenen Rentenansprüche zu verbessern (Infolge des Anreizes zu einer Leistungsausweitung verschlechtern sie womoglich gar die späteren Finanzierungsbedingungen.). 
Tragung der Lasten inner- oder außerhalb des Rentenversicherungssystems müssen die Mitglieder der nachfolgenden Generation in ihrer Aktivzeit eine höhere Summe des von ihnen erwirtschafteten Sozialprodukts an die Mitglieder ihrer Elterngeneration überweisen. ${ }^{259}$ Durch eine nicht durch Senkung anderweitiger Rentenansprüche gegenfinanzierte Kinderberücksichtigung steigt die Summe der monetarisierten Rentenansprüche.

Bei dem gerade Ausgeführten ist zu beachten, dass es im gegenwärtigen Rentensystem einen ,Sandwich-Effekt' gibt: Die verringerte durchschnittliche Kinderzahl auf Seiten der Mitglieder der Aktivgeneration fördert die Partizipation größerer Teile am Erwerbsleben, erhöht also tendenziell die Lohnsumme, und erleichtert die Aufbringung der zur Befriedigung der Rentenansprüche der gegenwärtigen Rentnergeneration aufzubringenden Mittel, wirkt kurzfristig also beitragssatzsenkend. ${ }^{260}$ (Insoweit ist die gerade geführte Diskussion, in der steigende Beitragssätze zur GRV mit der Lage auf dem Arbeitsmarkt begründet werden, zumindest leicht verzerrt: Die hierbei implizit unterstellte Referenzsituation, die auf einer Vollbeschäftigung aller gegenwärtigen Erwerbspersonen basiert, wird ihrerseits (u.a.) vom veränderten generativen Verhalten beeinflusst.) Den (infolge der Ausdehnung der Lohnsumme) höheren heutigen Beitragszahlungen resp. niedrigen Beitragssätzen steht im gegenwärtigen Rentenversicherungssystem eine Ausweitung der späteren Rentenansprüche gegenüber, noch dazu bei zweifach verringerter Möglichkeit zur Befriedigung dieser Ansprüche: Zum einen infolge eines verringerten Umfangs der nachfolgenden Generation, zum anderen durch sinkende Möglichkeit dieser Generation, die Rentenzahlungen durch Ausweitung des Anteils der Erwerbstätigen an der Aktivgeneration zu erbringen - die (potenziellen) Reserven des Systems werden schon jetzt weitgehend ausgeschöpft.

Es stellt sich zusätzlich noch die Frage nach der Sinnhaftigkeit einer Kinderberücksichtigung in Form von pauschalierten Beitragszeiten. Ist es das Ziel der Kinderberücksichtigung, die Opportunitätskosten des Kinderhabens zu senken, so wäre es von daher angemessen, die entfallenden Beitragszahlungen nicht pauschaliert zu ersetzen, sondern in Anlehnung an das Einkommensprofil des betroffenen Versicherten. Denn während die ,Vergütung' mit 1,0 persönlichen Entgeltpunkten pro Jahr für Angehörige niedrigerer Einkommenskategorien die unmittelbaren rentenmäßigen Konsequenzen einer dreijährigen Arbeitsunterbrechung zugunsten der Kindererziehung kompensiert bis überkompensiert, ist dies für (potenziell)

259 Und zwar ohne, dass es systemimmanent infolge der Regelung zu einer Ausweitung des Sozialproduktes, die eine Tragung grőßerer Lasten durch die Nachfolgegeneration erleichtert, kommt.

Vgl. DINKEL (1984a), S. 104. 
Besserverdienende nicht der Fall. Wenn das Ziel der Kinderberücksichtigung in der RV hingegen darin liegt, es den Versicherten zu erleichtern, sich für die drei ersten Lebensjahre ganz um das Kind kümmern zu können, ohne deswegen auf eine ,angemessene' (verglichen mit Nicht-Kindererziehenden) Altersabsicherung verzichten zu müssen, so ist eine Bewertung der Erziehungszeit mit Entgeltpunkten in jeweils individueller Bemessung der angemessenere Weg. Über die exakte Höhe der ,Bepunktung' lässt sich hierbei streiten: So könnte die Zeit mit der Punktzahl im Jahr vor der Arbeitsunterbrechung bewertet werden (denn diese Zahl entspricht ungefähr dem kurzfristig entstehenden Versicherungsausfall), oder aber mit der Jahrespunktzahl am Ende der Berufstätigkeit (denn bei Karriereverzicht und Senioritätsentlohnung entspricht diese Punktzahl am ehesten der Punktzahl, die durch die Kindererziehung entfällt), oder mit der durchschnittlichen Punktzahl in der Phase der aktiven Beitragszahlung aus abhängiger Beschäftigung. Letzteres hätte den Vorteil, Schwankungen in der jeweiligen Einkommensposition während der Aktivzeit zu berücksichtigen. Aber auch bei dieser Methode senken aus der Unterbrechung der Erwerbstätigkeit resultierende Karrierenachteile (mit einhergehenden geringeren Beitragszahlungen) die Rentenhöhe im Alter.

Außerdem ist zu hinterfragen, ob die RV der angemessene Ort für die Berücksichtigung der Opportunitätskosten des Kinderhabens ist. Die niedrigeren Rentenansprüche bei Kindererziehung sind Folge der Unterbrechung der Erwerbstätigkeit bzw. der verringerten Beitragszahlungen. Das Kinderhaben bzw. ein temporärer Verzicht auf Erwerbstätigkeit sind per se kein Versicherungsfall und von daher weder rentenbegründend, noch die Höhe der Rente gegenüber anderweitig begründeten Verdienstausfällen ändernd. Wenn der Staat Opportunitätskosten des Kinderhabens ausgleichen will, dann erscheint es zumindest angemessener, statt einer ,Bepunktung' der beitragsfreien Zeit das Geld, das bei freiwilliger Beitragszahlung in die GRV eine Steigerung der persönlichen Entgeltpunkte um 3,0 induziert hätte, an die Eltern auszuzahlen. ${ }^{261}$ Hierbei erhalten die Eltern des Kindes die Verfügungsmöglichkeit über das Geld in ihrer Aktivzeit, können sich einen zusätzlichen ,Rentenanspruch' durch freiwillige Zahlungen in die GRV erkaufen, müssen dies aber nicht; ihre Möglichkeit zur Optimierung des intertemporalen Konsumplans wird verbessert. Der verringerte Zwangskonsum der Anlageform $\mathrm{RV}$ bedeutet einen Zugewinn an ökonomischer Freiheit.

Die z.Z. bestehende Regelung hat eine gewisse pro-natalistische Tendenz, ohne dass diese aus rentenversicherungsimmanenten Gründen in dieser undifferenzierten Form zu rechtfertigen wäre. Sie hat dafür aber den Vorteil, einfach handhab-

${ }^{261}$ Fur einen in diesem Sinne noch weitergehenden Ansatz vgl. HACKMANN (1990). 
bar zu sein und nur eines geringen Kontrollaufwands zu bedürfen. Ein politischer Hauptvorteil dürfte aber darin bestehen, der Forderung des Bundesverfassungsgerichts nach (stärkerer) Berücksichtigung der Bedeutung des Kinderhabens für die RV einfach entsprechen zu können. ${ }^{262}$ Dies gilt gleich in zweifacher Art und Weise: Zum einen wird vermieden, die Kosten der aktuellen Kinderförderung unmittelbar kenntlich zu machen. Zwar werden sie inzwischen - in Form eines Bundeszuschusses zur GRV - ausgewiesen. Die Finanzmittel können aber sofort zur Finanzierung gegenwärtiger Renten eingesetzt werden. ${ }^{263}$ Zum anderen lässt sich auf dem bisher beschrittenen Weg eine sukzessive Erhöhung der finanziellen Berücksichtigung von Erziehungsleistungen vornehmen (z.B. nach jeweiligen Urteilen des Bundesverfassungsgerichts ...), ohne das politisch sensible Thema einer fundamentalen Umgestaltung des Rentensystems angehen zu müssen. Zwar muss jede Änderung der Zahlungsströme der RV hin in Richtung einer stärkeren Anerkennung von Erziehungsleistungen (c.p.) zu einer Verringerung der Renten ,Kinderarmer' führen, doch wird dies im gegenwärtigen System - infolge der Ausgestaltung - kaum deutlich. Kurzfristig profitieren (c.p.) möglicherweise sogar die gegenwärtigen Bezieher beitragsbedingter Renten von einer stärkeren Anerkennung der Kindererziehung - infolge der zusätzlichen Finanzmittel durch den erhöhten Bundeszuschuss.

Die Einschätzung LÜDEKEs, „[b]ei konsequenter Anwendung einer solchen Verknüpfung [,zwischen dem privaten Tragen der Kinderlasten und dem späteren Rentenempfang ..., wie sie seit einigen Jahren ansatzweise durch die Anrechnung von Erziehungszeiten in der Gesetzlichen Rentenversicherung geschieht ${ }^{“ 264}$ ] gehör[e] die niedrige Reproduktionsrate vielleicht schon recht schnell der Vergangenheit $a^{6 / 265}$, erscheint - sowohl infolge des Umfangs der Kinderberücksichtigung, als auch infolge ihrer Ausgestaltung - zu optimistisch. ${ }^{266}$ Was allerdings,

${ }^{262} \mathrm{Vgl}$. Anhang 5 (Abschnitt 7.5).

${ }^{263}$ Die versicherungsmathematisch bei gegebenem , aktuellen Rentenwert' eines Entgeltpunktes zu berechnenden Forderungen aus der ,Bepunktung' von Kindererziehungszeiten werden - wie der Barwert der übrigen Rentenansprüche auch - nicht in der ,Vermögensbilanz der Rentenversicherung (...)' ausgewiesen!

LÜDEKE (1995), S. 153.

LÜDEKE (1995), S. 153.

${ }^{266}$ So ganz sicher scheint LÜDEKE sich seiner Sache nicht zu sein - immerhin fährt er im direkt daran anschließenden Satz fort: „Ins Blickfeld gerät so die Möglichkeit, bei sinkender Geburtenziffer - und damit einhergehender Entlastung einer Generation vom Aufziehen von Kindern - verstärkt via Kapitalakkumulation Vorsorge für das Alter zu betreiben, um so die zusätzliche Belastung späterer Erwerbstätigengenerationen durch die umlagefinanzierte Rentenversicherung in Grenzen zu halten" (LÜDEKE (1995), S. 153 - Hervorhebungen durch den Verfasser). 
unabhängig von jeder Wirkung einer, kinderorientierten' Änderung der RV auf die Fertilität, für eine Umgestaltung spricht, sind Aspekte der intragenerationalen Verteilungsgerechtigkeit. ${ }^{267}$

\subsubsection{Weitere Elemente der Kinderberücksichtigung im deutschen Rentenrecht}

Die bisherigen Ausführungen haben sich ausschließlich mit der Berücksichtigung von Kindern beim Bezug einer Altersrente beschäftigt. Eine derartige Schwerpunktsetzung ist auch geboten, wird die überragende Bedeutung der Zielsetzung der Sicherung eines ,angemessenen' Lebensstandards nach altersbedingter Beendigung der Erwerbstätigkeit anerkannt. Dies ändert aber nichts daran, dass eine wesentliche Aufgabe der Rentenversicherung auch darin besteht, die Hinterbliebenen eines verstorbenen Erwerbstätigen finanziell abzusichern - und ferner auch den Versicherten selbst im Falle von erheblich verringerten Verdienstmöglichkeiten infolge körperlicher Gebrechen. ${ }^{268}$

\subsubsection{1. ,Aktive' Kinderberücksichtigung}

Zuerst soll auf eine Berücksichtigung des Kinderhabens im Rahmen ,allgemeiner' Ausgestaltungsregelungen der GRV eingegangen werden. Die zu betrachtenden Regelungen steigern zwar nicht die Höhe der Regelaltersrente, führen aber bei Eintritt anderer Versicherungsfälle (möglicherweise) zu einer Erhöhung der Leistungen.

\subsection{Waisenrente}

An erster Stelle zu nennen ist hierbei die Abhängigkeit der Höhe der Hinterbliebenenrente im Falle des Todes des Versicherten von der Zahl seiner Kinder; hier-

${ }^{267}$ Vgl. auch SCHMIDT et al. (1985), S. 45.

${ }^{268}$ Wobei mit Wirkung vom 01.01.2001 Renten wegen Berufsunfähigkeit und Renten wegen Erwerbsunfähigkeit durch eine ,Rente wegen Erwerbsminderung' ersetzt werden (vgl. SGB VI $\S 43$, Fassung bis zum 31.12.2000 / Fassung ab dem 01.01.2001; SGB VI $\S 44$ (,Rente wegen Erwerbsunfähigkeit') wird mit Wirkung zum 01.01.2001 aufgehoben.). Ohne auf Einzelheiten einzugehen kann festgehalten werden, dass es hierbei tendenziell zur Verschärfung der Anforderungen für die Gewăhrung einer Rente gegebener Hohe kommt. 
bei werden allerdings nur die vom Verstorbenen - in typisierter Hinsicht - wirtschaftlich abhängigen Kinder berücksichtigt. ${ }^{269}$

Die Höhe einer Halbwaisenrente beträgt 10 v.H. des Betrages, den der verstorbene Versicherte selbst erhielt (bei bereits erfolgendem Rentenbezug) bzw. bei Eintritt von Erwerbsunfähigkeit zum Zeitpunkt seines Todes erhalten hätte; der entsprechende Wert für eine Vollwaisenrente beträgt $20 \mathrm{v} . \mathrm{H} .{ }^{270}$

Die Waisenrente dient einer finanziellen Absicherung von Kindern des Verstorbenen. Ihre vergleichsweise geringe Höhe, die kaum zur Bestreitung des Lebensunterhaltes des Kindes ausreichen dürfte (die durchschnittliche Höhe der Waisenrente betrug $1996 \mathrm{ca}$. DM 300 / Monat) ${ }^{271}$ kann als Indiz dafür gelten, dass die Zahlungen der Rentenversicherung für sich genommen nicht zum Unterhalt des jeweiligen Kindes ausreichen sollen, sondern auf eine Ergänzung durch weitere staatliche Leistungen (Kindergeld, Sozialhilfe, Zahlungen im Rahmen des Bundesausbildungsförderungsgesetzes etc. $)^{272}-$ sowie einen Rückgriff auf Einkom-

269 Anspruch auf Waisenrente besteht nur bis zur Vollendung des 18. Lebensjahres, daruber hinaus längstens bis zur Vollendung des 27. Lebensjahres bei erfolgender schulischer- oder beruflicher Ausbildung (und im Falle einer Behinderung etc.; bei abgeleistetem Wehr- / Zivildienst erhöht sich die Obergrenze um den Zeitraum dieses Dienstes). Vgl. SGB VI § 48 (4).

${ }^{270}$ Vgl. SGB VI § 67. Zur Erklärung ist darauf hinzuweisen, dass sich die monatliche Rentenzahlung errechnen lässt aus dem Produkt der unter Berücksichtigung des Zugangsfaktors ermittelten persönlichen Entgeltpunkte mit dem aktuellen Rentenwert und dem Rentenartenfaktor. Die erste Komponente dient der Berücksichtigung der persönlichen durchschnittlichen Arbeitseinkommensposition des Versicherten während seiner Aktivzeit (wobei es zu Kürzungen bei Renteneintritt vor dem 65. Lebensjahr und zu Zuschlägen bei später erfolgendem Renteneintritt kommt - was sich im jeweiligen Zugangsfaktor widerspiegelt) und die zweite der Bestimmung der absoluten Rentenhöhe (Es wird der Betrag, den ein Bezieher von Altersrente, der mit 65 Jahren in den Ruhestand getreten ist, für ein Jahr, in dem er auf das durchschnittliche Arbeitseinkommen der in diesem Jahr abhängig Beschäftigten Beitragszahlungen zur GRV geleistet hat, an Rente erhält, festgelegt. Vgl. SGB VI § 68.). Der Rentenartenfaktor beträgt für Renten wegen Alters, Erwerbsunfähigkeit (bzw. ab 01.01.2001 wegen voller Erwerbsminderung) und Erziehungsrenten 1,0, für Halbwaisenrenten 0,1 und für Vollwaisenrenten 0,2 (vgl. SGB VI $\S 67)$.

${ }^{271}$ Vgl. STATISTISCHES BUNDESAMT, Statistisches Jahrbuch 1997, S. 472, Tab. 19.4.10. Zu dem ausgewiesenen Wert kommen noch die darauf entfallenden (von der GRV zu tragenden) Beiträge zur Kranken- und Pflegeversicherung.

272 Wobei die aufgefuhrten Unterstützungsleistungen unterschiedliche Anspruchsvoraussetzungen haben - insbesondere erfolgen Zahlungen im Rahmen der Sozialhilfe nur bei Bedürftigkeit, so dass eine niedrige Waisenrente bei höherer Rente des verbliebenen Elternteils oder vorhandenem Vermögen nicht mit dem Bezug von Sozialhilfe einhergeht. 
men und Vermögen des noch lebenden Elternteils (incl. einer eventuellen Witwen-/ Witwer- oder Erziehungsrente) - angelegt sind. ${ }^{273}$

\subsection{Witwen-, Witwer- und Erziehungsrente}

Kinder werden im Rahmen der Rentenversicherung auch bei der Festlegung der Höhe der Witwenrente berücksichtigt: ${ }^{274}$ Wird vom (nicht wieder verheirateten) Ehepartner des verstorbenen Versicherten ,ein eigenes Kind oder ein Kind des versicherten Ehegatten, das das 18. Lebensjahr noch nicht vollendet hat ${ }^{6275}$, erzogen, so besteht Anspruch auf die sog. große Witwen- bzw. Witwerrente. ${ }^{276}$ Diese beträgt 60 v.H. des Rentenbetrags, den der Verstorbene bei Eintritt von Erwerbsunfähigkeit zum Zeitpunkt seines Todes gehabt hätte, während die sonst gewährte sog. kleine Witwenrente 25 v.H. des oben bezeichneten Wertes ausmacht. ${ }^{277}$

Die große Witwenrente soll es dem verbliebenen Elternteil ermöglichen, die Erziehung des Kindes fortzuführen. Allerdings ist zur Relativierung der Bedeutung der großen Witwenrente anzumerken, dass nach Vollendung des 45 . Lebensjahres jeder (nicht wieder verheiratete) hinterbliebene Ehepartner eines Versicherten unabhängig davon, ob Kinder erzogen werden resp. wurden - Anspruch auf Bezug der großen Witwenrente besitzt. ${ }^{278}$

Eine vergleichbare Regelung gibt es auch für geschiedene (und nicht wieder verheiratete) Ehepartner eines Verstorbenen, die (mindesten) ein Kind betreuen: Sie haben Anspruch auf eine sog. Erziehungsrente. ${ }^{279}$ Die Regelung hat zur Folge, dass ein Unterhaltsberechtigter infolge des Todes des Unterhaltsverpflichteten

${ }^{273}$ Zur Steigerung der Witwenrente und zur Gewährung von ,Erziehungsrente‘ vgl. den nachfolgenden Abschnitt 5.2.2.1.2.

${ }^{274}$ Im Folgenden wird aus sprachlichen Grunden nur auf den Fall ,Witwenrente' eingegangen die Aussagen gelten entsprechend auch für Witwerrenten.

275 SGB VI $§ 46(2)$.

${ }^{276}$ Vgl. SGB VI $§ 46(2)$.

277 Vgl. SGB VI $§ 67$.

278 Vgl. SGB VI $§ 46$.

${ }^{279}$ Vgl. SGB VI § 47. Der ,Rentenartenfaktor' für Erziehungsrenten beträgt 1,0 (Vgl. SGB VI $\S 67$, Pkt. 4). 
nicht gezwungen ist, die Kindererziehung zugunsten einer Erwerbstätigkeit einzuschränken, um dadurch den Unterhalt zu sichern.

Beide Regelungen erleichtern die Entscheidung, für Kinder und Kindererziehung (zeitlich begrenzt) auf eine Erwerbstätigkeit zu verzichten. Der Zwang zu kurzfristiger (und ,überraschender') Arbeitsübernahme dürfte regelmäßig mit besonderen Problemen verbunden sein. ${ }^{280}$ Ohne Absicherung für den Fall des Todes des (ehemaligen) Ehepartners wäre somit eine (zumindest teilweise) Erwerbstätigkeit beider Elternteile sinnvoll, um so mögliche Probleme eines (kurzfristigen) (Wieder-)Einstiegs in den Arbeitsmarkt zu vermeiden.

\subsection{Sonderformen der Kinderberücksichtigung}

Am Ende dieses Abschnitts sollen noch einige weitere Formen der Kinderberücksichtigung im Rahmen der GRV erwähnt werden: Das Übergangsgeld bei medizinischen und berufsfördernden Leistungen und „bei Arbeitslosigkeit im Anschluß an berufsfördernde Maßnahmen"281 erhöht sich, wenn der Versicherte ein Kind betreut. ${ }^{282}$ Ebenso kann, wenn ein Kind unter 12 Jahren (oder ein behindertes Kind) im Haushalt wohnt, für die Zeit, in der die Versicherte auf Grund einer „medizinischen, berufsfördernden oder sonstigen Leistung[..] ${ }^{\text {‘283 }}$ außer Haus untergebracht, ist eine Haushaltshilfe auf Kosten der Rentenversicherung gestellt werden. ${ }^{284}$

Die Kinderberücksichtigung dient hierbei der Ermöglichung der Partizipation von Elternteilen an von der Rentenversicherung finanzierten Maßnahmen zur Steigerung der Chancen des Betroffenen auf dem Arbeitsmarkt. Ob die Rentenversicherung der richtige (alleinige) Träger von solchen Aufwendungen ist, ist zu bezwei-

${ }^{280} \mathrm{Zu}$ nennen sind hierbei z.B. die Organisation der Versorgung der Kinder und ein vergleichsweise niedriger Lohn infolge geringer Mobilităt und anderer für den Arbeitgeber eher unattraktiver Eigenschaften des Betroffenen, wie z.B. eines höheren Anspruchs auf Sonderurlaub zur Betreuung kranker Kinder.

${ }^{281}$ SGB VI $\S 24(1) \mathrm{Nr} .1$.

${ }^{282}$ Vgl. SGB VI § 24 (1). Die Leistungen der GRV erhöhen sich durch das Vorhandensein eines Kindes von 75, 70 bzw. 63 v.H. „der maßgebenden Berechnungsgrundlage” (ebd.) auf 90, 80 bzw. 68 v.H.

${ }^{283}$ SGB VI $§ 29(1)^{1}$.

${ }^{284}$ Vgl. SGB VI $\S 29(1)^{1}$. Voraussetzung hierfür ist, dass „eine andere im Haushalt lebende Person den Haushalt nicht weiterfuhren kann“" (ebd., Nr. 2). 
feln, liegen hier doch eher allgemeine Probleme der Vereinbarkeit von häuslicher Abwesenheit infolge berufsbedingter Anforderungen und Kindererziehung vor, die - wenn Hilfsbedarf konstatiert wird - zumindest partiell aus dem allgemeinen Steueraufkommen zu finanzieren sind. Am eventuellen Erfolg einer solchen Maßnahme profitiert immerhin auch die Gemeinschaft der Steuerzahler in Form von höheren steuerbaren Einkünften. Allerdings ist eine vergleichsweise ,intensivere' Partizipation der Mitglieder der Rentenversicherung am Erfolg der Weiterbildung gegeben, so dass eine zumindest partielle Zurechnung des Betreuungsaufwands zum Träger ,GRV' begründbar erscheint.

\subsubsection{2. ,Passive' Kinderberücksichtigung}

$\S 57$ des Sechsten Sozialgesetzbuches legt fest, dass „[d]ie Zeit der Erziehung eines Kindes bis zu dessen vollendetem zehnten Lebensjahr[..] .. bei einem Elternteil eine Berücksichtigungszeit [ist], soweit die Voraussetzungen für die Anrechnung einer Kindererziehungszeit auch in dieser Zeit vorliegen"285,286. Zeiten wiederum, ,in denen Versicherte ... wegen Schwangerschaft oder Mutterschaft während der Schutzfristen nach dem Mutterschutzgesetz eine versicherte Beschäftigung oder selbständige Tätigkeit nicht ausgeübt haben"287,288, zählen als Anrechnungszeit. ${ }^{289}$ Die Auswirkungen dieser besonderen Berücksichtigung von ,Zeiten', die mit der Kindesgeburt zusammenhängen, ergeben sich aus ihrer Bewertung (d.h. der eventuellen Zurechnung von Entgeltpunkten): Alle gerade genannten ,Zeitvergaben' verhindern, dass sich eine Kindererziehung rentenmindernd auswirkt.

Die Berücksichtigungszeit ist nur für die Gesamtleistungsbewertung bedeutsam. ${ }^{290}$ Bei dieser werden für die berücksichtigungsfähigen Zeiten der Kindererziehung

${ }^{285}$ SGB VI $§ 57$ - Hervorhebung durch den Verfasser.

${ }^{286}$ Die im Gesetz erwähnten Voraussetzungen brauchen im Rahmen der vorliegenden Arbeit nicht weiter $\mathrm{zu}$ interessieren.

${ }^{287}$ SGB VI $§ 58(1)^{1}$.

${ }^{288}$ Das Mutterschutzgesetz (MuSchG) hält (u.a.) fest, dass „,[w]erdende Mütter .. in den letzten sechs Wochen vor der Entbindung nicht beschäftigt werden [dürfen], es sei denn, daß sie sich zur Arbeitsleistung ausdrucklich bereit erklären" (MuSchG $\S 3$ (2)). Außerdem dürfen Frauen 8 Wochen nach der Entbindung (bei Frühgeburten 12 Wochen) nicht beschäftigt werden (vgl. MuSchG §6(1)).

${ }^{289} \mathrm{Vgl}$. SGB VI $\S 58(1)^{1}$.

${ }^{290} \mathrm{Vgl.} \mathrm{SGB}$ VI $§ 71$ (3). 
„jedem Kalendermonat ... die Entgeltpunkte zugeordnet, die sich ergeben würden, wenn diese Kalendermonate Kindererziehungszeiten wären ${ }^{\text {“291 }}$. Die Gesamtleistungsbewertung ist entscheidend für die Zurechnung persönlicher Entgeltpunkte für beitragsfreie Zeiten. Letztere erhalten nämlich den Durchschnittswert der persönlichen Entgeltpunkte im ,belegungsfähigen Zeitraum' ${ }^{292,293}$ Damit bei dieser Berechnung Zeiten der Kindesbetreuung (die 3 Jahre, für die eine Kindererziehungszeit angerechnet werden, überschreiten) nicht zu einer Senkung der durchschnittlichen Zahl der Entgeltpunkte und somit zu einer (deutlichen) Verringerung der Zurechnung für beitragsfreie Zeiten führen, werden den infolge einer Kindererziehung beitragsfreien Zeiten die oben bezeichneten Entgeltpunktwerte ,zugerechnet'.

Die Bedeutung der Gesamtleistungsbewertung besteht darin, dass bei Tod des Versicherten (für Witwen- und Waisenrenten), Eintritt einer ,rentenberechtigenden' Erwerbsminderung und bei ,Erziehungsrenten' die sich aus den bisherigen Beitragszahlungen (und ,Kinderanrechnungen') des Versicherten ergebende Zahl der persönlichen Entgeltpunkte aufgestockt wird durch zugerechnete Entgeltpunkte für die Zeit zwischen dem Eintritt der Erwerbsunfähigkeit und dem Augenblick der Vollendung des 60. Lebensjahres. ${ }^{294}$

Die besondere Behandlung von Zeiten, in denen sich ein Elternteil um ein bis zu 10 (aber mehr als 3) Jahre altes Kind kümmert, hat zur Folge, dass sich dieser Verzicht auf Erwerbstätigkeit im Falle einer Minderung der Erwerbsfähigkeit nicht in einer drastischen Reduktion der Absicherung im Falle eines vorzeitigen Eintritts des Versicherungsfalls niederschlägt. Sie dient mithin der Anpassung der Ausgestaltung des Versicherungsschutzes für Nicht-Altersrenten an die in Abhängigkeit von eventueller Kindererziehung unterschiedlich strukturierte Erwerbsbiographien, und erleichtert es einem Elternteil, zugunsten der Kindererziehung zumindest zeitweise (und über die drei ersten Lebensjahre des Kindes hinaus) auf eine Erwerbstätigkeit zu verzichten, wird doch der Verlust an Absicherung im Falle vorzeitigen Renteneintritts verringert.

${ }^{291}$ SGB VI § $71(3)$.

${ }^{292}$ Vgl. SGB VI $\S 71(1)^{1}$.

293 Der belegungsfähige Gesamtzeitraum wird in SGB VI $\S 72(2)$ definiert. Er beginnt mit der Vollendung des 16. Lebensjahres und geht - im Regelfall - bis zum Renteneintritt.

${ }^{294}$ Vgl. SGB VI § 59. Die Zeit bis zur Vollendung des 55. Lebensjahres wird in vollem Umfang, die darüber hinausgehende Zeit zu einem Drittel zugerechnet. 
Entsprechendes gilt auch für die Regelung, dass eine Berücksichtigungszeit wegen Kindererziehung den erforderlichen Zeitraum vor Zahlung einer Rente wegen Berufs- bzw. Erwerbsunfähigkeit, in dem Pflichtbeiträge gezahlt worden sein müssen, mindert. ${ }^{295}$

\subsubsection{Allgemeine Bewertung}

Bei der Bewertung der Kinderberücksichtigung im Rahmen der gegenwärtigen GRV stellt sich die Frage nach dem geeigneten Referenzmaßstab. Ganz allgemein lässt sich festhalten, dass Mitglieder der Rentenversicherung, die von ihnen wirtschaftlich abhängige Kinder haben, für die Rentenversicherung (c.p.) schlechtere Risiken darstellen: Die erwarteten Zahlungen an Versicherte mit Kindern sind höher als die an kinderlose Versicherte. Viele (potenzielle) Leistungen der GRV können von kinderlosen Versicherten nicht in Anspruch genommen werden. Zwar lässt sich eine argumentative Gegenposition vorstellen, eine ,soziale' Rentenversicherung sei eben immer und definitionsgemäß mit (gewollter!) Umverteilung verbunden, und die ,Vorteile' Kinderhabender seien dementsprechend nur natürlich, doch ändert eine solche Argumentation nichts daran, dass es im Rahmen der gegenwärtigen Ausgestaltung der GRV - insbesondere im Bereich der Hinterbliebenenabsicherung - verglichen mit dem Referenzmaßstab risikoäquivalenter Beiträge zur GRV eine Begünstigung Kinderhabender gibt.

\subsubsection{Berücksichtigung verlängerter Rentenbezugsdauer}

Da die deutsche Rentenversicherung einer Sicherung des Lebensstandards im Alter dient, wobei dieses Ziel relativ zum Lebensstandard eines repräsentativen Mitglieds der Aktivgeneration definiert ist, hat eine Änderung des Zahlenverhältnisses zwischen Rentnern und Beitragszahlern Auswirkungen auf die von den Beitragszahlern im Durchschnitt zu leistenden Beiträge. ${ }^{296}$ Das gerade bezeichnete Zahlenverhältnis wird von verschiedenen Faktoren bestimmt: im Zähler von der Abgrenzung der Gruppe der Rentenberechtigten, der durchschnittlichen Zahl der Kinder sowie deren Bereitschaft (und Fähigkeit) zur Beitragszahlung etc.

Die Bedeutung des Rückgangs der durchschnittlichen Zahl der Kinder der Mitglieder der Rentnergeneration für die Fähigkeit der Rentenversicherung, Leistungen an die Rentnergeneration sicherzustellen, ist bereits in Abschnitt 3.3.3.2 de-

${ }^{295} \mathrm{Vgl}$. SGB VI $\S 43(3), 44$ (4).

${ }^{296} \mathrm{Zu}$ den genaueren Wirkungen vgl. die grundsătzlichen Ausführungen in Abschnitt 3.3.3.2. 
tailliert untersucht worden. Die dortige Argumentation befasst sich, wird (vereinfacht) ein (qualitativer), Rentnerlastquotient' definiert als das Verhältnis zwischen der Zahl der Rentner und der Zahl der Beitragszahler, mit der ,Nennergröße' ${ }^{297}$

Doch auch die ,Zählergröße' ist für die Belastung der Aktivgeneration durch Beitragszahlungen zur Rentenversicherung bedeutsam - ihre Erhöhung bedeutet (c.p.) eine zunehmende Zahlungsverpflichtung der Mitglieder der Aktivgeneration.

Im Folgenden soll die Berücksichtigung zweier verschiedener Faktoren, die auf die Belastung der Mitglieder der Aktivgeneration durch Beitragsverpflichtungen zur Rentenversicherung einwirken, im Rahmen des deutschen Rentenrechts thematisiert werden. Hierzu werden zuerst jeweils die beiden Problemlagen charakterisiert, und der jeweilige gesetzliche Lösungsansatz skizziert, um dann die beiden Verfahren miteinander zu vergleichen und zu hinterfragen, inwieweit die Ausgestaltung als Beitrag zur Sicherung der ,Überlebensfähigkeit' der Rentenversicherung gegenüber der ,klassischen' demographischen Verschiebung, nämlich dem Rückgang der durchschnittlichen Kinderzahl, anzusehen ist.

\subsubsection{1. Änderung der Abgrenzung der Rentenberechtigten}

Die erste Möglichkeit zur ,zählerseitigen' Einflussnahme auf den Rentnerlastquotienten besteht in einer Änderung der Abgrenzung der zum jeweiligen Zeitpunkt Rentenberechtigten (im weiteren kurz: Rentner). Durch Änderungen in den Bedingungen für den Rentenbezug kann deren Zahl variiert werden. Insbesondere hat das durchschnittliche Alter bei erstmaligem Rentenbezug Bedeutung: Je eher der Renteneintritt im Durchschnitt erfolgt, desto höher ist (c.p.) zu jedem Augenblick die Zahl der Rentner (der repräsentative Rentner hat dann eine längere Rentenbezugsphase, womit zu jedem Augenblick ein größerer Teil der Bevölkerung Rentner ist). ${ }^{298}$ Der gerade beschriebene Mechanismus ist aber auch deshalb be-

297 Die hier gewählte Abgrenzung weicht von der ,gewöhnlichen' insoweit ab, als sie nicht auf Basis des jeweiligen Alters der Personen erfolgt. Für die im weiteren erfolgende Thematisierung erscheint die hier erfolgende Abgrenzung sinnvoller, da ein möglicher Parameter zur Minderung der ,rentenmäßigen' Last einer Alterung der Gesellschaft gerade darin besteht, den Anteil z.B. der 65jährigen, die noch erwerbstätig sind, an der Gruppe der 65jährigen zu erhöhen.

298 Für die weitere Argumentation ist es dabei nicht bedeutsam, ob ein ,offizielles Rentenalter' abgesenkt wird, ab dem erst ,normale' Altersrente bezogen werden kann, oder ob das festgelegte Renteneintrittsalter unverändert bleibt, infolge veränderter anderer Regelungen (oder Umgebungsbedingungen) jedoch ein häufigerer Renteneintritt schon vor dieser Zeit erfolgt; wichtig ist hier nur, dass das durchschnittliche Alter bei erstmaligem Rentenbezug faktisch ab- 
deutsam, weil u.U. durch Ausweitung der Zahl der Rentner parallel die Zahl der (potenziellen) Beitragszahler verringert wird, so dass auch von daher der Rentnerlastquotient zunimmt. ${ }^{299}$

In den letzten Jahren ist im Rahmen der Rentenversicherung ein Abschlag bei der monatlichen Rentenhöhe bei Eintritt in die Rente vor Erreichen des ,Regelalters' eingeführt worden. Für jeden Monat, den der erste Rentenbezug einer Altersrente (bei Männern) vor dem 65. Lebensjahr liegt, erfolgt ein Abschlag von 0,3 v.H. der normalen monatlichen Rente; maximal können so 5 Jahre ,angerechnet ' werden mit einem Abschlag von 18 v.H. Erfolgt der Renteneintritt bei Männern erst nach dem 65. Lebensjahr, so wird für jeden Monat 0,5 v.H. auf den Rentenzahlbetrag aufgeschlagen. ${ }^{300,301}$ Folge dieser Regelung ist, dass der Erwartungswert des Barwertes der Rentenzahlungen an einen Rentner nur in geringem Maße von seiner Entscheidung über den Augenblick des erstmaligen Bezugs von Renteneinkommen abhängt. ${ }^{302}$ Gelingt die Korrektur genau, so steigt durch frühzeitigen Renten-

nimmt. (In einem erweiterten Analyserahmen wäre auf eine nicht entsprechend abgesenkte durchschnittliche monatliche Rentenhöhe hinzuweisen.)

299 Während das offizielle Alter für den erstmaligen Bezug von Regelaltersrente in Deutschland bei 65 Jahren für Männer liegt, und bei Frauen zur Zeit sukzessive von 60 auf 65 Jahre angehoben wird, beträgt das durchschnittliche Zugangsalter fur Versichertenrenten im Jahre 1998 für die alten Bundesländer 60,2 und fur die neuen Bundesländer 58,1 Jahre (für Deutschland insgesamt 59,8 Jahre) (vgl. VERBAND DEUTSCHER RENTENVERSICHERUNGSTRÄGER (1999), S. 101). Wird nur auf ,Renten wegen Alters' abgezielt, so betrăgt das durchschnittliche Zugangsalter 62,4 für Männer und 62,6 furr Frauen in den alten Bundesländern, 61,0 bzw. 60,4 in den neuen Bundesländern und 62,1 bzw. 62,2 in Deutschland (vgl. ebd., S. 104). (Das höhere Rentenzugangsalter von Frauen ist wohl zum einen Folge einer geringeren Beschäftigung in Berufen mit ungewöhnlich frühem durchschnittlichen Renteneintritt, sowie einer infolge von Unterbrechungen der Erwerbstätigkeit erst späteren Erfullung von Mindestbeitragszeiten.)

${ }^{300}$ Für dieses und das vorangegangene vgl. SGB VI $§ 77$ (2). Zur Feststellung, dass diese Abbzw- Zuschläge nicht versicherungsmathematisch äquivalent sind, und somit Anreiz zur Frühverrentung geben, vgl. BÖRSCH-SUPAN (1992).

${ }^{301}$ Die Regelungen für Frauen sind entsprechend, wobei der Referenzpunkt für die Berechnung der jeweiligen Ab- bzw. Zuschläge infolge der Anpassung des ,Regelalters' fur den Renteneintritt von Frauen sukzessive verschoben wird.

${ }^{302}$ Die vorher gültige Regelung ohne derartige Ab- bzw. Zuschläge hatte eine eindeutige Subventionierung fruhzeitigen Renteneintritts zur Folge, also ein deutliche Verzerrung der Entscheidung über den Zeitpunkt des Renteneintritts. Bei versicherungsmathematisch ,exakten' Abund Zuschlägen wäre eine solche Verzerrung nicht mehr gegeben. Problem hierbei ist, inwieweit die ,Grunde' für einen vergleichsweise frühen bzw. späten Bezug der Regelaltersrente bei der Bestimmung des Ab- bzw. Zuschlags berucksichtigt werden sollen: Hier ist mit Selbstselektion zu rechnen, so dass diejenigen, die sich für einen späteren Renteneintritt entscheiden, 
eintritt zwar die Zahl der Rentner, die kumulierte Rentenhöhe, die die Rentner auf Dauer beziehen, bleibt dabei aber unverändert. Es wird mithin (idealtypisch) dafür gesorgt, dass die finanzielle Belastung der nachfolgenden Generation im Rahmen des Rentensystems nicht von Abweichungen des faktischen Renteneintrittsalters der Mitglieder der Aktivgeneration vom gesetzlich fixierten normalen Erstbezugsalter einer Regelaltersrente abhängt.

\subsubsection{Rentenkürzung durch einen, demographischen Faktor'}

Wurde bisher eine, individuelle' Verlängerung der Dauer des Rentenbezugs durch vergleichsweise frühen Eintritt in die Rentenphase betrachtet, so wird jetzt die andere Determinante der Zahl der Rentner untersucht: eine Verlängerung der durchschnittlichen Restlebenserwartung bei Erreichen des ,normalen' gesetzlichen Alters für den erstmaligem Bezug der Altersrente. ${ }^{303}$

Nimmt die durchschnittliche Restlebenserwartung derjenigen, die das 65. Lebensjahr (als das gesetzlich vorgegebene normale Eintrittsalter für den Bezug einer Regelaltersrente) erreichen, zu, ohne dass die Grenze für den Bezug einer Regelaltersrente entsprechend angehoben wird, so steigt mithin auch die durchschnittliche Rentenbezugsdauer, und somit auch der Rentnerlastquotient. Um die aus der zunehmenden Restlebenserwartung der 65jährigen resultierenden Beitragsbelastungen der Beitragszahler abzumildern, ${ }^{304}$ wurde in der letzten Legisla-

im Durchschnitt wohl auch eine überdurchschnittliche Lebensdauer haben, und somit bei gegebenem Renteneintrittsalter eine im Durchschnitt ungewöhnlich lange Rentenbezugsdauer (Umgekehrt lässt sich für ,kränkelnde' Versicherte, die sich für einen frühen Renteneintritt entscheiden, argumentieren.).

${ }^{303}$ Während die durchschnittliche Bezugsdauer einer Versichertenrente bei Mănnern (Frauen) in den alten Bundesländern im Jahre 1980 11,0 (13,8) Jahre betrug, liegt sie im Jahre 1998 bei 14,2 (18,1) Jahren. Für die neuen Bundesländer lautet der entsprechende Wert für 1998 11,3 $(19,7)$ Jahre (vgl. VERBAND DEUTSCHER RENTENVERSICHERUNGSTRÄGER (1999), S. 121). (In den gerade genannten Werten sind allerdings die Folgen fruheren durchschnittlichen Renteneintritts enthalten!) Das durchschnittliche Wegfallsalter ist in Westdeutschland von 68,3 Jahren im Jahre 1960 uber 72,7 Jahren (1980) auf 75,6 Jahren (1998) gestiegen (vgl. ebd., S. 115).

304 NB: Es geht nicht um die Berücksichtigung von Änderungen der durchschnittlichen Lebenserwartung ,Neugeborener' oder eines repräsentativen Mitglieds der Gruppe der Rentenbezieher, sondern um die entsprechende Änderung der Restlebensdauer beim Erreichen des gesetzlich vorgegebenen Alters zum Erstbezug einer Regelaltersrente! Diese Unterscheidung ist insoweit wichtig, da ubereinstimmende Änderungen der durchschnittlichen Lebenserwartung unterschiedliche Folgen für die Rentenversicherung haben können: Steigt die durchschnittliche Lebenserwartung infolge erhöter Wahrscheinlichkeit eines Lebendgeborenen, das 65. Lebensjahr zu erreichen, so entlastet dies die Rentenversicherung tendenziell. Steigt sie jedoch aus- 
turperiode der von CDU/ CSU und FDP gestellten Bundesregierung unter HELMUT KoHL eine ,demographische Komponente' in die Rentenformel eingeführt. ${ }^{305}$ Ziel dieser Komponente ist es, die durch Verlängerung der Restlebenserwartung der 65jährigen entstehenden zusätzlichen Beitragsbelastungen quasi in zwei Teile zu gliedern: Die eine Hälfte dieser zusätzlichen Belastung der RV soll weiterhin von den Beitragszahlern in Form höherer Beitragszahlungen (Beitragssätze) zur Rentenversicherung aufgebracht werden. Die andere Hälfte hingegen soll von den jeweiligen Rentnern getragen werden - in Form einer entsprechenden Kürzung der jeweiligen monatlichen Rentenzahlung.

Der zuletzt genannte Teil entspricht in seiner rechnerischen Logik dem bei ,frühzeitigem' Renteneintritt angewandten Verfahren, einen gegebenen Barwert der kumulierten monatlichen Rentenzahlungen auf einen längeren Bezugszeitraum zu strecken, indem die jeweilige monatliche Rentenzahlung entsprechend gekürzt wird. Der erstgenannte Teil hingegen bedeutet eine Hinnahme einer gesteigerten Belastung der Beitragszahler.

Die hier skizzierte Logik einer ,hälftigen Teilung' der finanziellen Lasten einer zunehmenden Lebensdauer im Rahmen der Rentenversicherung wird allerdings durchbrochen. In SGB VI $\S 68$ (6) ist nämlich festgelegt: „Der Faktor für die Veränderung der durchschnittlichen Lebenserwartung der 65jährigen ist nicht anzuwenden, soweit seine Anwendung zu einer Verringerung ... [bisheriger Renten] oder zu einem geringeren Verhältniswert aus einer jahresdurchschnittlichen verfügbaren Standardrente und dem Nettoentgelt nach der Volkswirtschaftlichen Gesamtrechnung als 64 vom Hundert führt". Hat eine erhöhte (Rest-)Lebenserwartung der Rentenbezieher also infolge der ,Lastverteilungslogik' eine (vermeintlich) zu starke Kürzung des Rentenniveaus zur Folge, so wird die diesbezügliche Tarifkomponente nicht berücksichtigt, und die finanziellen Lasten einer steigenden Lebenserwartung werden (wieder) primär von den Aktiven getragen.$^{306}$

Das gerade vorgestellte Verfahren sollte ursprünglich zum 01.01.1999 in Kraft treten, doch wurde dieser Termin nach dem erfolgten Regierungswechsel zu einer von SPD und BÜNDNIS ' 90 -DIE GRÜNEN getragenen Bundesregierung unter

schließlich deswegen, weil die durchschnittliche Restlebensdauer 65jăhriger zunimmt, so belastet dies (c.p.) die Rentenversicherung.

${ }^{305} \mathrm{Vgl}$. SGB VI $§ 68$.

${ }^{306}$ Da das Netto-Rentenniveau im Jahre 1999 bei ca. 70,5 v.H. liegt (vgl. VDR (1999), S. 208), ist der Augenblick des erstmaligen ,Greifens' dieser Regelung kaum abzusehen. Sie informiert eher über ein längerfristig angepeiltes Absicherungsniveau. 
GERHARD SCHRÖDER auf den 01.01.2001 verschoben; da bis dahin eine allgemeine ,Rentenreform' geplant ist, erscheint es zweifelhaft, ob die getroffene Regelung jemals Anwendung finden wird.

\subsubsection{Vergleich der unterschiedlichen Ansätze}

Beide gerade skizzierten Ansätze dienen dazu, die sich aus einer steigenden durchschnittlichen Rentenbezugsdauer ergebenden Belastungen der Mitglieder der Aktivgeneration abzumildern bzw. zu vermeiden. Während beim ersten Verfahren das (idealtypische) Ziel ist, eine Belastung der Mitglieder der Aktivgeneration durch die individuellen Entscheidungen über den erstmaligen Rentenbezug zu verhindern, ${ }^{307}$ wird beim zweiten Ansatz ,nur' eine Absenkung der Anstiegs der Belastung der nachfolgenden Aktivgeneration um 50 v.H. angestrebt. Das zweite Verfahren ist insoweit, altenfreundlicher'.

Zwischen den beiden betrachteten Ursachen einer erhöhten ,Rentenlast' besteht ein Unterschied: Während einmal eine individuelle Entscheidung über einen vergleichsweise frühen oder späten Renteneintritt im Rahmen der gesetzlichen Bestimmungen vorliegt, ist das zweite ein kollektives Phänomen, nämlich die $\mathrm{Zu}-$ nahme der Restlebensdauer 65jähriger. Insoweit mag eine alleinige Tragung der finanziellen Konsequenzen frühzeitigen Renteneintritts durch den jeweiligen Versicherten bei gleichzeitig breiter Tragung der finanziellen Lasten allgemein gesteigerter Lebenserwartung konsequent sein. ${ }^{308}$ Auch sprechen - wegen der unterschiedlichen Rolle des individuellen Entscheidungsverhaltens - allokative Gesichtspunkte nicht gegen eine Differenzierung.

In beiden Fällen wird aber - wie schon erwähnt - primär auf den ,Zähler' des Rentnerlastquotienten abgezielt, d.h. Änderungen des Verhältnisses zwischen der Zahl der Rentner und der Zahl der Aktiven, die sich aus einem Rückgang des Umfangs der nachfolgenden Generation ergeben, werden nicht betrachtet. ${ }^{309}$ Es

${ }^{307}$ Hierbei wird aber auch eine Entlastung der Aktiven infolge eines späteren Rentenerstbezugs verhindert.

${ }^{308}$ Die Zunahme der durchschnittlichen Restlebensdauer kann auch Folge der Ausgestaltung des deutschen Sozialversicherungssystems sein: $\mathrm{Zu}$ den Anreizwirkungen einer Rentenversicherung mit periodischen Zahlungen hin zu einer ,Lebensverlängerung' (zumal in Verbindung mit einer fur den Patienten weitgehend kostenfreien Gesundheitsversorgung) vgl. PHILIPSON und BECKER (1998).

${ }^{309}$ Infolge der demographischen Verschiebung bzw. wegen der von dieser ausgehenden Belastung der Rentenversicherung gewinnt die zusätzliche Belastung durch zunehmende Restlebenserwartung der Versicherten bei Renteneintritt eines besondere Bedeutung. 
kommt mithin nicht zu einer Internalisierung der externen Effekte verringerter Kinderzahlen. Die in den weiteren Kapiteln folgenden Ausführungen zu den Belastungswirkungen eines abnehmenden Generationenumfangs sowie zur Sinnhaftigkeit einer Berücksichtigung von Kindern im Rahmen einer umlagefinanzierten Rentenversicherung etc. werden durch die eingeführten bzw. geplanten ,demographischen' Komponenten nicht redundant.

\subsubsection{Exkurs: Nähe von ,Frauenförderung' zu ,Kinderförderung'?}

Eine Begünstigung von Frauen findet sich in den Anspruchsvoraussetzungen für Altersrenten, bei denen Frauen (unter nicht allzu restriktiven Bedingungen) schon mit dem 60. Lebensjahr in Rente gehen können (bei 65 Jahren als Bedingung für die Regelaltersrente bei Männern). ${ }^{310}$ Obendrein werden Frauen durch einen geschlechtsunabhängigen Beitragssatz infolge ihrer höheren (Rest-)Lebenserwartung bei Renteneintritt gegenüber Männern bevorzugt: Ihr Erwartungswert der Rentenzahlungen bei gegebenem Wert der Beitragszahlungen ist höher. Eine Berücksichtigung der Tatsache, dass Frauen für die GRV ,schlechte Risiken' darstellen, wäre ohne nennenswerten (Kontroll-)Aufwand möglich, so dass die Tatsache einer unterlassenen Differenzierung auf Beitrags- und / oder Rentenseite eindeutig als Bevorzugung von Frauen anzusehen ist. Dies alles gibt Anlass zu fragen, ob die in der Rentenversicherung an mehreren Stelle eindeutig festzustellende Bevorzugung von Frauen gegenüber Männern als implizite Kinderberücksichtigung angesehen werden kann.

Ein unmittelbarer Bezug der gerade skizzierten Regelungen zum Kinderhaben und zur Kindererziehung besteht nicht: Auch kinderlose Frauen profitieren von der Ausgestaltung der GRV, so dass von einer eineindeutigen Kinderförderung auf dem Weg der Frauenförderung sicher nicht gesprochen werden kann. Allerdings hat die Unterscheidung zwischen den beiden Ansatzpunkten ,Frau' und ,Mutter' im Zeitablauf an Bedeutung gewonnen. Bei Verabschiedung der Regelung war der Anteil der Frauen, die keine Kinder hatten, deutlich geringer als zur heutigen Zeit. Somit hat die ,Treffgenauigkeit' einer eventuellen Kinderförderung auf dem (Um-)Weg über eine Frauenbegünstigung mit der Zeit abgenommen.

In diesem Zusammenhang ist beachtenswert, dass die Regel-Altersgrenze für nach dem 31. Dezember 1939 geborene Frauen jetzt sukzessive an den Wert für Män-

${ }^{310} \mathrm{Zu}$ den einzelnen Anspruchsvoraussetzungen für Renten wegen Alters (für ,Normalfälle', d.h. nicht unter Tage Beschăftigte etc.) vgl. SGB VI $§ \S 35$ - 39. 
ner (65 Jahre) angeglichen wird. ${ }^{311}$ Die Unterschiede in der durchschnittlichen Rentenbezugsdauer sollten sich mithin in Zukunft verringern. Eine vollständige Abschaffung der Begünstigung von Frauen findet auf diese Weise aber nicht statt, denn auf Grund der höheren (Rest-)Lebenserwartung bleiben Frauen - bei andauerndem Verzicht auf , geschlechtsspezifische' Beitragssätze - (c.p.) für die GRV auch nach dieser Änderung ein ,schlechteres Risiko' als Männer.

\subsubsection{Zum Ausmaß der Kinderberücksichtigung im Rahmen der GRV}

Um die Bedeutung der gegenwärtigen Kinderberücksichtigung im Rahmen der GRV zu verdeutlichen, wird im Folgenden kurz auf Abschätzungen der Summe der ,kinderbezogenen' Zahlungen im Rahmen der GRV und ihres Anteils an den Gesamtausgaben eingegangen. Sollen diese ermittelt werden, ergeben sich allerdings verschiedene Probleme: Das erste - und wesentliche - besteht darin, dass die Angaben der Rentenversicherungsträger immer auf Ein- und Auszahlungen sowie Fallzahlen abstellen. Es gibt mithin keine Statistik der akkumulierten Rentenansprüche. Dies mag für ein Umlageverfahren, das durch eine Stromgrößengleichung (nämlich die - prinzipielle - Übereinstimmung von Ein- und Auszahlungen) charakterisiert ist, und bei dem es keine Rücklagen gibt, systemisch angemessen sein: Grundsätzlich gibt es dann keine die Einzahlungen übersteigenden Auszahlungsverpflichtungen. Da aber in der Praxis zumindest das Ziel einer Erhaltung des nominalen aktuellen Rentenniveaus vorherrscht, und ferner allgemein ein Bestandsschutz an ausgewiesenen Renten besteht, ${ }^{312}$ so dass ein Anstieg der Rentenansprüche (c.p.) zumindest in Teilen zu einer Erhöhung des Beitragssatzes führt, ${ }^{313}$ ist eine solche Argumentation - wiewohl formal korrekt - material fragwürdig. Zumindest aber wäre der Ausweis einer Summe der bestehenden Rentenansprüche bei gegebener Ausgestaltung der Rentenversicherung, wobei die Berechnung dieser Summe die durchschnittliche (Rest-)Lebenserwartung der Versicherten berücksichtigt, sinnvoll.

Offengelegt werden die kinderbezogenen Rentenansprüche, die schon heute zu Zahlungen an Rentner führen. Da aber der Umfang der Kinderberücksichtigung,

${ }^{311}$ Vgl. SGB VI § 41 i.V.m. Anlage 20 zum SGB VI.

312 Das Bundesverfassungsgericht hat allerdings festgehalten, dass im Zuge einer Verbesserung der Kinderberücksichtigung im Rahmen der GRV eine maßvolle Senkung bestehender Rentenanspriliche nicht verfassungswidrig wäre.

${ }^{313}$ Oder aber zu einer Ausweitung der Bemessungsgrundlage. 


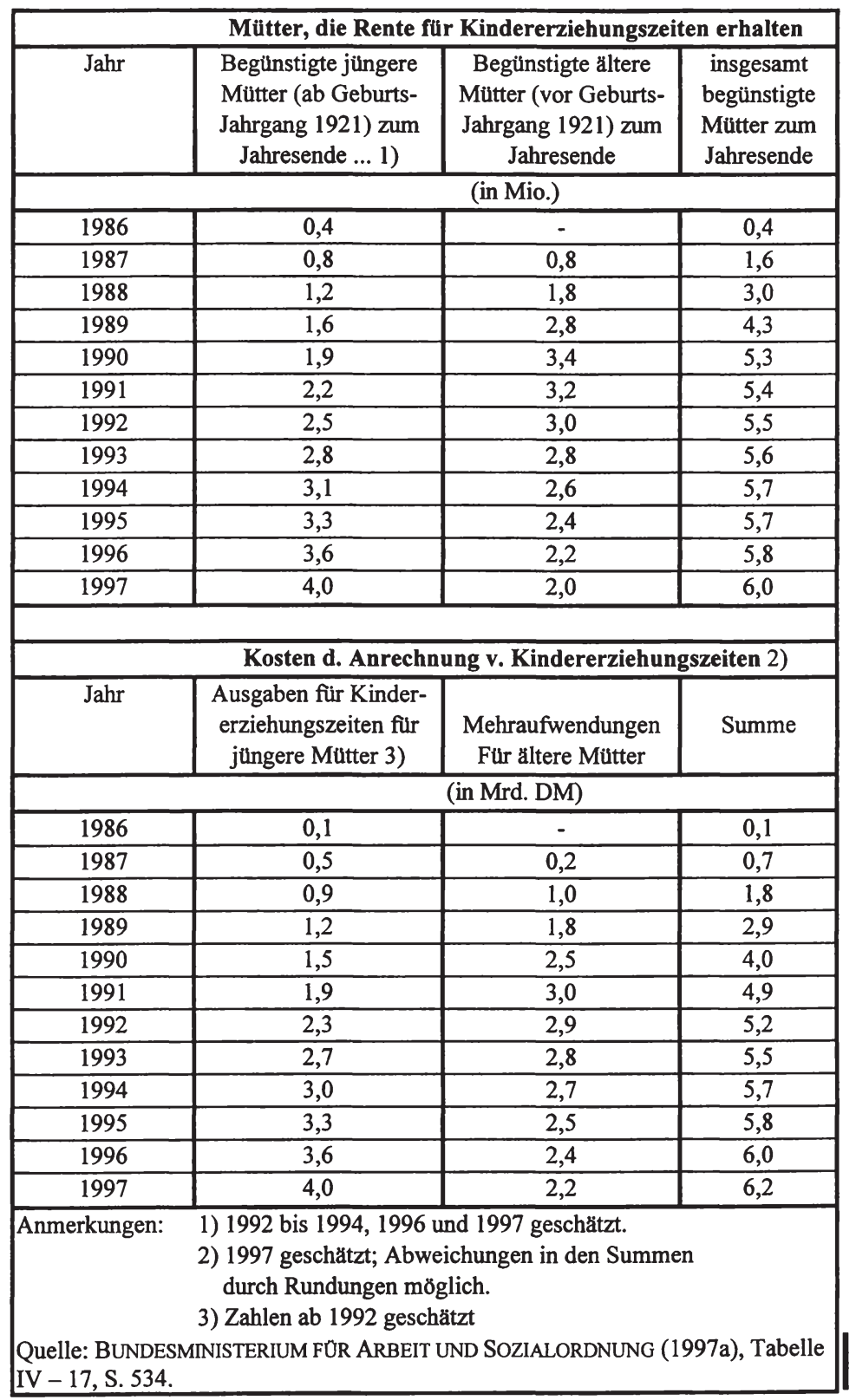

Tabelle 4: Auswirkungen der Anerkennung der Kindererziehung im Rentenrecht in den alten Bundesländern seit 1986 
d.h. der rentenmäßige Wert eines Kindes, seit der Einführung einer rentensteigernden Wirkung des Kinderhabens sukzessive erhöht worden ist, führen die in Tabelle 4 ausgewiesenen Werte zu einer Unterschätzung der (späteren) finanziellen Belastungen der Rentenkasse. Bei der Bewertung der absoluten Kosten der Anrechnung von Kindererziehungsleistungen ist zu berücksichtigen, dass das Gesamtausgabenvolumen der Gesetzlichen Rentenversicherung im Jahre 1997 (für Gesamtdeutschland) bei ca. DM 438 Mrd. lag - ${ }^{314}$ der Anteil der ,kindererziehungszeit'-bedingten Zahlungen betrug weniger als 1,5 v.H. (Zum Vergleich: Im Zeitraum von 1986 bis 1997 sind zusammen ca. DM 49 Mrd. für Renten wegen Kindererziehungszeiten ausgegeben worden.)

Während sich die finanziellen Folge der kinderbedingten Steigerungen der Rentenansprüche nach dem heutigen Rentenrecht grob abschätzen lassen, sind entsprechende Angaben für andere Formen der Kinderberücksichtigung kaum zu errechnen. Die Kinderberücksichtigung manifestiert sich hier z.B. in der Höhe einer Hinterbliebenenrente (vgl. die Ausführungen zur ,Gesamtleistungsbewertung' in Abschnitt 5.2.2.2), wobei die vorliegenden Statistiken keine Abtrennung eines ,kinderbedingten' Teils erlauben.

Eher kann eine Abschätzung für die Waisenrenten vorgenommen werden: Der Anteil dieser Zahlungen am Gesamtvolumen aller Rentenzahlungen ist recht gering. Dies folgt schon aus der - verglichen mit Alters- und Witwenrenten - niedrigen absoluten Höhe der Waisenrenten (Im Jahre 1991 lag ihr durchschnittlicher Wert in der Rentenversicherung der Arbeiter bei DM 263, bei der der Angestellten betrug er DM 289; die durchschnittliche Versicherten- bzw. Witwenrente belief sich auf DM 946 / 1337 (der erstgenannte Wert bezieht sich auf Arbeiter, der zweitgenannte auf Angestellte) bzw. DM 805 / 1109.). Hinzu kommt die geringe Fallzahl (Im Jahre 1990 waren von 9,149 Millionen laufenden Renten der RV der Arbeiter nur 211.000 Waisenrenten; bei der RV der Angestellten lauteten die Werte: 119.000 Waisenrenten bei insgesamt 5,195 Millionen Rentenfällen). ${ }^{315}$ Daraus lässt sich das Gesamtvolumen der Waisenrenten in der GRV für das Jahr 1991 mit ca. DM 1,079 Mrd. (zuzüglich den darauf entfallenden Zahlungen der GRV an die Krankenversicherung der Rentner) abschätzen. Daten über Fälle, in denen die Anspruchsvoraussetzungen für den Bezug einer, großen Witwenrente'

${ }^{314}$ Vgl. BUNDESMINISTERIUM FÜR ARBEIT UND SOZIALORDNUNG (1997a), Tabelle III, S. 332.

${ }^{315}$ Vgl. Statistisches BundeSAMt, Statistisches Jahrbuch 1991, Tab. 19.4.2, Tab. 19.4.8, S. $466 / 468$. Für eine grobe Abschätzung der parallel zu den durch Kindererziehungszeiten erworbenen beitragsbezogenen Rentenansprliche, und somit zu einer Einordnung der finanziellen Bedeutung der Kinderberücksichtigung im Rahmen der gegenwärtigen Ausgestaltung der Rentenversicherung, vgl. Anhang 5 (Abschnitt 7.5). 
auf Seiten der Hinterbliebenen ausschließlich als Folge des Kinderhabens erfüllt werden, liegen nicht vor.

Die vergleichsweise geringe Bedeutung von Erziehungsrenten zeigt sich (schon) in ihrer Anzahl: Im Jahr 1996 gab es in Westdeutschland 3.501 Erziehungsrenten beziehende Frauen und 1.330 Männer, die jeweiligen Werte für Ostdeutschland betrugen 3.195 resp. $161 .{ }^{316}$

Die direkte Berücksichtigung von Kindern bei der Festlegung der Rentenhöhe ist in den letzten Jahren deutlich ausgebaut worden. Durch die sukzessive Einführung in Verbindung mit einer weitgehenden Gültigkeit dieser ausgeweiteten Leistungen ,nur' für nach 1992 geborene Kinder hält sich die gegenwärtige finanzielle Belastung der Rentenversicherung in engen Grenzen. Die heute gewährten kinderbezogenen Rentenansprüche werden erst in einigen Jahren ihre kassenwirksame Dynamik entfalten. Die inzwischen erfolgenden Zahlungen aus dem Bundeshaushalt, die dem Ausgleich für die neu angerechneten ,Entgeltpunkte für Kindererziehungszeiten' dienen, führen kurzfristig sogar zu einer Erhöhung der gegenwärtig bezogenen Renten (sei es durch Verringerung des Drucks hin zu einer Rentensenkung, sei es durch einen vergleichsweise niedrigen Beitragssatz zur Rentenversicherung, der infolge der nettolohnorientierten Rentenanpassung nicht nur die gegenwärtigen Beitragszahler entlastet, sondern auch eine höhere Rente induziert). Die gegenwärtigen Bundeszuschüsse stellen für die Rentenversicherung den für ein Umlageverfahren charakteristischen ,Einführungsgewinn' dar; ihre beitragssenkende resp. rentensteigernde Wirkung lässt in dem Maße nach, wie der Umfang der kinderinduzierten Rentenzahlungen zunimmt, es also zu einer Angleichung der gegenwärtig entstehenden kinderbedingten Rentenansprüche und der parallel zu befriedigenden kinderbedingten Rentenverpflichtungen kommt.

Wird von den unmittelbaren rentensteigernden Wirkungen der Kinderberücksichtigung abstrahiert, so sind die kinderbezogenen Leistungen der Rentenversicherung vergleichsweise gering - und die Bedeutung dieser anderen Leistungen wird im Laufe der Zeit infolge eher abnehmender Zeiten der Erwerbsunterbrechung eines Elternteils zugunsten der Kindererziehung, sowie auf Grund verringerter absoluter Kinderzahlen und verringerter Kinderzahl pro repräsentativem aktiven Versicherten voraussichtlich weiter zurückgehen.

${ }^{316}$ Vgl. BUNDESRATS-DRUCKSACHE 552 / 97 vom 24.07.97, S. 112f., Übersicht I6. Einschränkend ist anzumerken, dass die der einzelnen Erziehungsrenten mit im Durchschnitt über DM 1.100 / Monat im Westen und knapp DM 1.100 / Monat im Osten verglichen mit Witwen- / Witwerrenten recht hoch sind (vgl. ebd., S. 116f., Übersicht I7). 
Im Folgenden sollen kurz zwei weitere Schätzansätze für den ,Grad der Beitragsorientierung' des gegenwärtigen deutschen Rentenrechts angegeben werden. Trotz der unterschiedlichen Abgrenzungen wird hier jeweils ein Grad der Beitragsorientierung von ca. $93-94$ v.H. ausgewiesen. ${ }^{317}$

In der Untersuchung in Kapitel 4 wurde zwischen beitrags- und kinderbezogenen Renten(ansprüchen) unterschieden; etwas Drittes gab es nicht. Für die folgende Abschätzung werden weiterhin Ausgaben der Rentenversicherung für Rehabilitationsmaßnahmen, aber auch Verwaltungskosten etc. implizit auf beide Teile der Rentenversicherung entsprechend ihrem jeweiligen Anteil an den restlichen Aufwendungen aufgeteilt. Für die aktuelle Abschätzung wird auf den für das Jahr 2000 vorgesehenen Bundeszuschuss für Kindererziehungszeiten Bezug genommen: Dieser beträgt DM 22,4 Mrd. ${ }^{318}$ In Zukunft soll die Rentenversicherung für jedes Kind 3 Jahre lang die Beitragszahlung erhalten, die der in der jeweiligen Periode auf ein durchschnittliches rentenversicherungspflichtiges Arbeitseinkommen anfallenden entspricht, also den ,Beitragswert' von 1,0 Entgeltpunkt.

Zur Abschätzung des Grades der Beitragsorientierung des bestehenden Rentenversicherungssystems (in der hiesigen Abgrenzung) ist der Bundeszuschuss für Kindererziehungsleistungen als Repräsentant der ,kinderbezogenen Leistungen' zu betrachten. Es stellt sich die Frage nach der richtigen Bezugsgröße für die gesamten Leistungen des Rentenversicherungssystems. Die Nutzung zweier verschiedener Möglichkeiten unterscheidet die beiden Schätzungen voneinander:

- Eine erste Abschätzung des Grades der ,Kinderorientierung' setzt den jährlichen kinderbezogenen Bundeszuschuss von DM 22,4 Mrd. ins Verhältnis zur Summe der Rentenzahlungen der Rentenversicherung der Arbeiter und Ange-

317 Es ist zu beachten, dass der Grad der Beitragsorientierung in der hier genutzten Form nur etwas über den Anteil der Summe nicht auf Grund von Kindererziehung der Versicherten entstandenen Renten(ansprüchen) an der Summe der gesamten Renten(ansprüche) innerhalb des bestehenden deutschen Rentenversicherungssystems aussagt - und nichts darüber, inwieweit innerhalb der , beitragsbezogenen' Rentenansprüche eine positive Beziehung zwischen dem jeweiligen Barwert der Beitragszahlungen eines Rentners und seinem Anteil an den beitragsbezogenen Renten(ansprüchen) besteht (d.h. eine Umverteilung innerhalb des beitragsbezogenen Rententeils, z.B. durch höhere Bewertung von Beitragsleistungen wăhrend der Ausbildung etc., wird als , beitragsbezogen' ausgewiesen).

318 Der entsprechende Wert für 1999 beträgt DM 13,6 Milliarden - er bezieht sich aber nur auf den Zeitraum vom 01. Juni (Inkraftreten des Gesetzes) bis zum 31. Dezember (vgl. SGB VI $\S 279$ ). In beiden Fällen ergibt sich mithin ein monatlicher Bundeszuschuss von ca. DM 1,9 Mrd. 
stellten. Für 1998 beträgt die letztere ca. DM 350,3 Mrd. ${ }^{319}$ Der auf dieser Basis errechnete Grad der Beitragsorientierung beläuft sich auf ca. 93,6 v.H.

- Bei der vorangegangenen Rechnung wurde der Grad der Beitragsorientierung abgeschätzt, indem heutige Beitragszahlungen für kinderbezogene Renten in Beziehung gesetzt wurden zur Summe der Rentenzahlungen des betreffenden Jahres. Dabei kommt es (jenseits unvermeidlicher technischer Schwierigkeiten der Abschätzung infolge der sich zur Zeit vollziehenden Umgestaltung des Rentenversicherungssystems) zu einer Vermischung unterschiedlicher ,Bezugsperioden': Die in der betrachteten Periode entstehenden kinderbezogenen Rentenansprüche werden in vorangegangenen Perioden entstandenen, aber in der betreffenden Periode gezahlten jährlichen Renten gegenübergestellt. Um dies zu vermeiden, bietet sich ein Vergleich mit den in derselben Periode (nach dem gegenwärtigen Recht) entstandenen beitragsbezogenen Rentenansprüchen an. Aus den gesamten Beitragszahlungen des Jahres 1998 für Westund Ostdeutschland von DM 255,6 Mrd. (West) bzw. DM 73 Mrd. (Ost), dem Beitragssatz zur Rentenversicherung der Arbeiter und Angestellten von 20,3 v.H. sowie der Bezugsgröße von DM 52.080 / Jahr für Westdeutschland bzw. DM 43.680 / Jahr für Ostdeutschland lässt sich eine in diesem Jahr beitragsbezogen erzielte kumulierte Summe der persönlichen Entgeltpunkte von ca. 24,177 Millionen im Westen und ca. 8,233 Millionen im Osten abschätzen. Da bis zur Fälligkeit dieser Rentenansprüche eine Angleichung des ost- an den westdeutschen ,aktuellen Rentenwert' unterstellt werden kann, erscheint eine Addition dieser beiden Punktwerte unproblematisch. 1998 wurden somit ca. 32,41 Millionen ,persönliche Entgeltpunkte' durch Beitragsleistungen erworben. Ein (für Gesamtdeutschland erfolgender) pauschalierter Bundeszuschuss für Kindererziehungszeiten von DM 22,4 Mrd. entspricht bei Nutzung der westdeutschen Bezugsgröße ca. 2,119 Millionen persönlichen Entgeltpunkten (und bei Nutzung der ostdeutschen Bezugsgröße ca. 2,526 Millionen persönlichen Entgeltpunkten) ${ }^{320}$. Der Anteil der durch Kindererziehungszeiten entstandenen Rentenansprüche an der Summe der insgesamt neu erworbenen Rentenansprüche eines Jahres beträgt mithin zwischen 6,1 und 7,2 v.H., für den Grad der Beitragsorientierung ergibt sich somit ein Wert von 92,8 bis 93,9 v.H. ${ }^{321,322}$

319 VERBAND DEUTSCHER RENTENVERSICHERUNGSTRÄGER (1999), S. 197f.; eigene Berechnung, der Wert enthält die Beitragszahlungen der GRV zur Krankenversicherung der Rentner.

22,3 Mrd. / (0,203*52.080) = (ca.) 2,119 Mill.

${ }^{321}$ Da z.Z. uber 80 v.H. aller Geburten in Westdeutschland zugerechnet werden, liegt der gesamtdeutsche Wert eher beim auf Basis der westdeutschen Bezugsgröße ermittelten Wert, und so- 
Obwohl in beiden vorangegangenen Abschätzungen der Grad der Beitragsorientierung eher unterschätzt wird (da der Bundeszuschuss für Kindererziehungszeiten des Jahres 2000 in Bezug gesetzt wird zu beitragsbezogenen Werten des Jahres 1998), ergibt sich jeweils ein Wert von fast 94 v.H. - nur ca. $6 \%$ aller Rentenzahlungen sind mithin unmittelbare Folge von angerechneten Jahren der Kindererziehung. Das deutschen Rentenversicherungssystem ist mithin als ausgesprochen beitragsorientiert zu kennzeichnen. Immerhin hat sich der Grad der Kinderorientierung in den letzten Jahren allerdings deutlich erhöht - von ca. 1,5 v.H. bei Betrachtung der gegenwärtigen Rentenzahlungen auf die gerade errechneten (ca.) 6 v.H. Anteil an den gegenwärtig entstehenden Rentenansprüchen.

mit der Grad der Beitragsorientierung des Rentenversicherungssystems im oberen Bereich der angegebenen Spanne.

${ }^{322}$ Für eine differenziertere Abschätzung der impliziten Kosten der Kinderberücksichtigung vgl. Anhang 4 (Abschnitt 7.4). Der sich dort - fur 1996 - ergebende Grad der Beitragsorientierung der GRV liegt bei ca. 93,3 v.H., also ziemlich genau in der Mitte der oben ausgewiesenen Schätzspanne. 


\section{Schlussbetrachtung}

Die folgende Schlussbetrachtung gliedert sich in zwei Teile: Zuerst wird hinterfragt, inwieweit eine - verglichen mit dem gegenwärtigen System in Deutschland - verstärkte Kinderberücksichtigung Auswirkungen auf die von der Bevölkerung realisierte durchschnittliche Kinderzahl haben kann. Daran anschließend erfolgt eine Diskussion der Chancen eines Ausbaus der Kinderberücksichtigung im politischen Prozess.

\subsection{Wie wahrscheinlich sind Änderungen des generativen Verhaltens bei verstärkter Kinderberücksichtigung im Rahmen der Rentenversicherung?}

In der bisherigen Abhandlung wurde auf einen deutlichen Rückgang der Kinderzahlen in Deutschland (und in vielen anderen Staaten insbesondere Europas) verwiesen, um dann die Bedeutung dieses Phänomens für eine umlagefinanzierte Rentenversicherung zu thematisieren. Anschließend erfolgte eine Analyse der distributiven Wirkungen einer (nicht) erfolgenden Kinderberücksichtigung im Rahmen der Rentenversicherung. Diese Untersuchung basierte auf gegenwärtigen Daten, insbesondere auch bezüglich der Kinderzahl. Es stellt sich somit die Frage, ob eine verstärkte Berücksichtigung generativer Leistungen bei der Rentenberechnung zu einer Änderung der durchschnittlichen Kinderzahl der Mitglieder der Aktivgeneration führen kann.

Die gerade aufgeworfene Frage ist schon deshalb bedeutsam, da jede Berücksichtigung von Erziehungsleistungen bei der Rentengewährung zur Folge hat, dass der Zusammenhang zwischen Beitragszahlungen und Rentenhöhe verringert wird: Der individuelle Steuercharakter von Beitragszahlungen zur GRV nimmt folglich $\mathrm{zu}$ - mit allen negativen Konsequenzen auf Anreizwirkungen für rentenversicherungspflichtige Tätigkeiten. ${ }^{323}$ Die Existenz einer nachfolgenden Generation ist zwar von entscheidender Bedeutung für die (Über-)Lebensfähigkeit eines Rentenversicherungssystems, aber Rentenzahlungen werden doch immer durch Beitragsleistungen der Aktiven finanziert. Dieses Problem tritt allerdings bei allen (nichtfreiwilligen) Leistungen zugunsten von Kindern oder ihren Eltern auf - auch

${ }^{323}$ Dies bedeutet nicht, dass die Gesamtwirkung der Kinderberlicksichtigung im Rahmen des Rentenversicherungssystems als allokativ schädlich zu beurteilen ist: Die mit den per se allokativ schädlichen Pflichtbeiträgen für die kinderbezogenen Leistungen der Rentenversicherung finanzierten Leistungen sind vielmehr allokativ vorteilhaft, verringern sie doch Fehlanreize hin in Richtung zu geringer Bildung von Humankapital. 
wenn diese über, gewöhnliche Steuern' finanziert werden: In real existierenden Steuersystemen, also unter Vernachlässigung einer alleinigen Mittelaufbringung über lump-sum-taxes - und bei weitgehender Ausschöpfung von Steuern zur Internalisierung negativer externer Effekte - kommt es infolge verzerrender Steuern zu Zusatzlasten der Besteuerung. Allokativ mag bestenfalls zu Gunsten einer Finanzierung über das allgemeine Steueraufkommen sprechen, dass die gegenüber der Rentenversicherungsabgabe breitere Bemessungsgrundlage eine vergleichsweise niedrigere Höhe des Steuersatzes zur Aufbringung der Finanzmittel erfordert, so dass die allokativen Zusatzlasten - wenn diese, wie allgemein unterstellt, mit der Höhe des Steuersatzes überproportional wachsen - insgesamt geringer sind (sowie ,breiter gestreut', was aus distributiven Gründen als vorteilhaft angesehen werden mag). Einschränkend ist allerdings anzumerken, dass in der gegenwärtigen Ausgestaltung der Rentenversicherung die allokative Vorteilhaftigkeit einer Finanzierung der Kinderberücksichtigung über die Einkommensteuer reduziert ist: Im Gegensatz zur Bemessungsgrundlage der Einkommensteuer gibt es bei der der Rentenversicherungsabgabe einen jährlichen Höchstbeitrag. Einkommen aus abhängiger Beschäftigung, das die Beitragsbemessungsgrenze übersteigt, wird nicht zur Finanzierung der Rentenversicherung herangezogen, so dass die Rentenversicherung im Bereich höheren Einkommens anreizmäßig unschädlich ist (der Rentenversicherungsbeitrag hat dann Pauschcharakter). Bei einer Finanzierung der rentenmäßigen Kinderberücksichtigung über die Einkommensteuer kommt es hingegen auch zu einer Belastung hoher Einkommen (sowie von Einkommen aus anderen Quellen als abhängiger Erwerbstätigkeit), die infolge der progressiven Ausgestaltung des Einkommensteuersystems sowieso schon überdurchschnittlich belastet sind.

Ein bedeutender Nachteil der Tragung der Kinderberücksichtigung aus dem allgemeinen Steueraufkommen ist der Verzicht auf die ostentative Darstellung der Tatsache, dass die Kindererziehung konstitutionelles Element einer umlagefinanzierten Rentenversicherung ist - und ihre finanzielle Anerkennung somit gerade keine versicherungsfremde Leistung.

Wenn aber mit der finanziellen Anerkennung der Bedeutung der Kindererziehung im Rahmen der Rentenversicherung schon negative Arbeitsanreizwirkungen verbunden sind, die tendenziell die Mittelaufbringung der zur ,Befriedigung' der Rentenansprüche der gegenwärtigen Rentner erschweren, so bleiben doch verschiedene Argumente, die trotz aller damit verbundenen Probleme zugunsten einer verstärkten Kinderberücksichtigung im Rahmen der GRV sprechen: $\mathrm{Zu}$ nennen wären zuerst distributive Gründe. Es ist zu beachten, dass eine Verbesserung der finanziellen Situation von Familien, wie sie insbesondere durch deren Entlastung schon in der Beitragsphase erzielt werden kann, über den unmittelbaren Einkommenseffekt und den Substitutionseffekt in Form einer Verringerung der peku- 
niären Kosten des einzelnen Kindes hinaus bedeutsam sein kann. Werden Kinder in geringerem $\mathrm{Maße}$ mit einem vergleichsweise niedrigen Lebensstandard assoziiert, so kann diese Verringerung der ,psychologischen Kosten ${ }^{6}$ des Kinderhabens ebenfalls das generative Verhalten beeinflussen.

Wenn Eltern (auch) eine Leistung zugunsten der Gemeinschaft der Rentenversicherten erbringen, dann kann es ein moralisches Gebot sein, dass sich die Versichertengemeinschaft auch an den Kosten der Kindererziehung - und zwar unmittelbar im Rahmen der GRV und nicht über bloße Partizipation an der Finanzierung eines allgemeinen Familienlastenausgleichs im Rahmen des Steuer- und Transfersystems - beteiligt. Die Dringlichkeit einer Verteilung der (finanziellen) Lasten der Kindererziehung auch auf Rentenversicherte mit unterdurchschnittlicher Kinderzahl kann dann die Inkaufnahme der mit der Mittelaufbringung einhergehenden Zusatzlasten rechtfertigen - wiewohl eine rationale Wirtschaftspolitik die allokativen Kosten immer beachten sollte, wenn es darum geht, distributive Zielsetzungen zu realisieren.

Weiterhin kann die explizite Anerkennung des Kinderhabens im Rahmen der GRV das Bewusstsein um die Bedeutung der Kindererziehung für die Aufrechterhaltung der Gesellschaft im Allgemeinen und der Rentenversicherung im Besonderen stärken.

Es bleibt noch auf die Frage einzugehen, inwieweit eine (gegenüber dem Statusquo verstärkte) Kinderberücksichtigung im Rahmen der GRV zu einer Steigerung der Geburtenzahl und einer Verbesserung des Ausbildungsstandes (Einkommenspotenzials) eines repräsentativen Mitglieds der nachfolgenden Generation beitragen kann - und somit zu einer Milderung der gegenwärtig (wohl) absehbaren Finanzierungsprobleme der GRV.

Eine eventuelle Umstellung hin zu einer stärkeren Berücksichtigung von Kindern ist ein langfristiger Prozess, insbesondere dann, wenn kurzfristig deutliche Änderungen bestehender Renten(ansprüche) vermieden werden sollen. Außerdem führt eine induzierte Steigerung der Geburtenhäufigkeit erst mit erheblicher Verzögerung zu einer möglichen Entlastung der Rentenversicherung. Da der Eintritt in das (rentenversicherungspflichtige) Berufsleben heute in vielen Fällen erst mit über 20 Jahren erfolgt, ist eine entsprechende ,Verzögerung' bezüglich eventueller Entlastungswirkungen sicher zu unterstellen - bei weiterer Steigerung der durchschnittlichen formalen Qualifikation nachwachsender Generationen eher noch eine Zunahme. Systemimmanent besteht sogar die Möglichkeit, dass ein Übergang zu einem Rentensystem, das die erfolgreiche Sozialisation von Kindern stärker rentensteigernd berücksichtigt, die Bemessungsgrundlage der Rentenversiche- 
rung erst einmal verringert: Gerade wenn die Geburtenziffer steigt, ist ein Rückgang der Zahl der Erwerbstätigen nicht auszuschließen - mit einhergehender Möglichkeit der Minderung der Summe der rentenversicherungspflichtigen Einkommen. Der schon erwähnte ,Sandwich-Effekt ${ }^{324}$ wirkt hier in die entgegengesetzte Richtung: Ein Rückgang der Summe der rentenversicherungspflichtigen Einkommen bei (wegen?) gleichzeitiger Steigerung der durchschnittlichen Kinderzahl der Mitglieder der Aktivgeneration erschwert die Befriedigung der Rentenansprüche der gegenwärtigen Rentnergeneration, erhöht aber tendenziell die ,Rendite' der Beitragszahlungen für die Mitglieder der gegenwärtigen Aktivgeneration. Insoweit könnte der Übergang zu einer stärker kinderberücksichtigenden Ausgestaltungsform der Rentenversicherung - jenseits aller ,technischen' Umstellungsprobleme - auch in seinen Anreizwirkungen in kürzerer und mittlerer Frist eher eine Verschärfung der Finanzierungsprobleme mit sich bringen: Sowohl die Anreizwirkungen hin $\mathrm{zu}$ einer Vermeidung rentenversicherungspflichtiger Erwerbstätigkeit infolge eines gesteigerten ,Steuercharakters' der Pflichtzahlungen zur Rentenversicherung, als auch die gerade geschilderten Wirkungen eines eventuellen Erfolgs der Ausgestaltung im Sinne einer Steigerung der Geburtenziffer bergen die Gefahr einer kurzfristigen Erosion der Finanzbasis der Rentenversicherung.

Damit aber ist zu fragen, ob eine stärker kinderorientierte Ausgestaltung der Rentenversicherung langfristig in der Lage ist, durch Setzung von Anreizen zur Steigerung der Humankapitalbasis eine Ausweitung der Finanzierungsbasis der Rentenversicherung zu fördern. Nehmen infolge der Reform die Investitionen in das Humankapital der nachfolgenden Generation zu, und verbreitert sich somit (vor allem auch nach Berücksichtigung der schon geschilderten Anreizwirkungen hin zu einer Vermeidung rentenversicherungspflichtiger Erwerbstätigkeit mit einhergehender Verringerung der Bemessungsgrundlage der GRV) c.p. per Saldo die Bemessungsgrundlage, was wiederum die weitere Funktionsfähigkeit stabilisiert? ${ }^{325}$ Erstaunlicherwiese wird in der Literatur bei den verschiedenen dort vorgeschlagenen Reformansätzen kaum auf diese doch bedeutsame Frage eingegan-

${ }^{324}$ Vgl. Dinkel (1984a), S. 104. Der ,Sandwich-Effekt" stellt sich in der bisherigen Betrachtungsweise wie folgt dar: Eine Erhöhung der Summe der rentenversicherungspflichtigen Einkommen bei (wegen?) gleichzeitiger Verringerung der durchschnittlichen Kinderzahl der Mitglieder der Aktivgeneration erleichtert (c.p.) die Befriedigung der Rentenansprüche der gegenwärtigen Rentnergeneration, verringert aber tendenziell die ,Rendite' der Beitragszahlungen für die Mitglieder der gegenwärtigen Aktivgeneration.

${ }^{325} \mathrm{Zu}$ beachten ist, dass die intergenerativen distributiven Wirkungen abhängig sind von der $\ddot{A} n$ derung der Wachstumsrate der Aktivgeneration bzw. der Summe der rentenversicherungspflichtigen Einkommen. 
gen. Dies mag damit zusammenhängen, dass jedwede Antwort auf die gestellte Frage spekulativ bleiben muss.

$\mathrm{Zu}$ bedenken ist, dass der Trend zu einer Verringerung der durchschnittlichen Kinderzahl der einheimischen Bevölkerung schon seit längerem besteht $-{ }^{326}$ insbesondere, wenn man die Zahl der das fortpflanzungsfähige Alter erreichenden Nachkommen betrachtet. Sicherlich ist dieser Trend keine ausschließliche Folge der Ausgestaltung der Rentenversicherung; somit lassen Änderungen im Rentenversicherungssystem nur eine recht geringe ,Korrektur' des generativen Verhaltens erwarten. ${ }^{327}$ Im Folgenden sollen verschiedene (potenzielle) Gründe für eine Abnahme der durchschnittlichen Kinderzahl vorgestellt werden, um dann kurz zu diskutieren, inwieweit eine, kinderfreundlichere' Ausgestaltung der GRV in der Lage sein könnte, die jeweiligen ,Hemmnisse' abzubauen.

Gerade wenn die Entscheidung über das generative Verhalten nicht ausschließlich unter (in einem engen Sinne), ökonomischen' Gesichtspunkten erfolgt, sind allgemeine Umgebungsbedingungen bzw. Stimmungen bedeutsam. Insbesondere ist zu beachten, dass der, soziale Druck' hin zu Heirat und ,Kinderhaben' im Zeitablauf abgenommen hat. ${ }^{328}$ Dies gilt umso mehr, als die Mitglieder der Elterngeneration auch schon einer verringerten diesbezüglichen Erwartungshaltung ausgesetzt waren. ${ }^{329}$ Ehe- und Kinderlosigkeit werden zunehmend als ,normale' Lebensformen angesehen. ${ }^{330}$

${ }^{326}$ Schon im Jahr 1933 wurde nur in Familien mit einem selbständigen Landwirt als Haushaltsvorstand und in Arbeiterfamilien eine furr den ,Populationserhalt' ausreichende Kinderzahl realisiert und bereits 1939 reichte die durchschnittliche Kinderzahl von 10 - 14 Jahre alten Ehen nicht mehr zum Erhalt des Generationenumfangs (vgl. SCHWARZ (1982), S. 582, Tab. 4; für weitere - entsprechende - Angaben vgl. auch SCHWARZ (1990b), S. 441). Frauen ab ca. dem Geburtsjahrgang 1885 haben eine durchschnittliche Zahl der Lebendgeborenen, die nicht mit einem Erhalt des Generationenumfangs vereinbar ist (vgl. SCHWARZ (1991), S. 154f., insbes. Tab. 2). Für eine Darstellung des Rückgangs der ehelichen Fruchtbarkeit in Deutschland von 1880 bis 1912, sowie weitere Angaben zu auf die Geburtenentwicklung einwirkenden Faktoren vgl. auch NIPPERDEY (1990), insbes. Kap. I und II.

${ }^{327}$ Insbesondere ist nicht davon auszugehen, dass eine ,populationserhaltende ' Geburtenziffer erreicht wird.

${ }^{328} \mathrm{Vgl}$. MOLT (1977) und ROSENSTIEL (1977). Es ist zu beachten, dass ein ,Wertewandel' sich vermutlich nicht ohne äußere Einflusse ergibt, sondern dass auch hier in Teilen, das Sein das Bewusstsein bestimmt'.

329 So konstatiert HÖFLINGER (1982, S. 22): „[E]s scheint, daß speziell die Entscheidung, ob man Kinder haben will oder kinderlos bleiben will, von sozialen Gegebenheiten des Ehepaares bestimmt wird, wogegen die Entscheidung fur oder ein drittes Kind sozial indeterminierter er- 
Parallel dazu hat das durchschnittliche Heiratsalter zugenommen, was, da die große Mehrheit der Kinder weiterhin ehelich geboren wird, ${ }^{331}$ schon aus biologischen Gründen mit einer Tendenz zu einer Verringerung der durchschnittlichen Kinderzahl innerhalb einer Ehe einhergeht. ${ }^{332}$

Zusätzlich ist die durchschnittliche Ausbildungsdauer - insbesondere von Frauen - deutlich gestiegen. Hiervon gehen mehrere Effekte aus: Zum einen ist ein unmittelbarer Institutionen- oder Partizipationseffekt zu nennen. ${ }^{333}$ Eine Ausbildung ist nur schwer mit gleichzeitiger Kindererziehung zu verbinden; eine weitere ,Verzögerung' des Zeitpunktes der Geburt des ersten Kindes ergibt sich dann obendrein daraus, dass sich auch in der Anfangsphase der Erwerbstätigkeit nach Beendigung der Ausbildung eine Unterbrechung durch die Nutzung eines ,Erziehungsurlaubs' regelmäßig für die berufliche Laufbahn nachteilig auswirkt. Die Hinauszögerung der Geburt eines ersten Kindes bedeutet aber auch, dass der zur Komplettierung der Familie zur Verfügung stehende Zeitraum schon aus biologischen Gründen kürzer wird, und die Wahrscheinlichkeit, Kinder höherer Ordnungszahlen zu haben, dementsprechend sinkt. ${ }^{334}$ Und dann bedeutet eine verlängerte Ausbildung eine Investition in das Humankapital: Durch Verzicht auf kurzfristigen Einkommenserwerb verbessert sich - im Durchschnitt - die Verdienstmöglichkeit nach Beendigung der Ausbildung. Die gestiegenen Reallohnsätze nach Steuern (und ,steueräquivalenten Abgaben') erhöhen die Opportunitätskos-

scheint. ... Die Entscheidung fur oder gegen ein zweites Kind wird zum einen von der Geschwisterzahl des Ehemannes beeinflußt. ... Wăhrend der Ausbildungsstatus mit dem Wunsch nach einem zweiten Kind positiv assoziiert ist, ist das Einkommen negativ mit dem Wunsch nach einem zweiten Kind assoziiert." (Auch mit der Zahl der Geschwister einer verheirateten Frau nimmt die Wahrscheinlichkeit, dass sie keine Kinder oder (nur) ein Kind hat, deutlich ab; vgl. RÜCKERT (1976).)

${ }^{330}$ Vgl. SCHMID (1988), S. 126 - 128.

${ }^{331}$ Wobei in den neuen Bundesländern 1994 ca. 50 v.H. der ledigen Frauen im Alter von 35 - 39 Jahren mit einem Kind in einem gemeinsamen Haushalt wohnen, wăhrend der entsprechende Wert fur das ehemalige Bundesgebiet deutlich unter 20 v.H. liegt (vgl. DORBRITZ und SCHWARZ (1996), S. 237, Tab. 3).

${ }^{332}$ Für eine Darstellung des Zusammenhangs zwischen Heiratsalter der Frau und Wahrscheinlichkeit der Kinderlosigkeit vgl. DORBRITZ und SCHWARZ (1996), S. 248, Tab. 15, PROEBSTING (1983), S. 862f. und SCHWARZ (1988).

${ }^{333}$ Vgl. BlOSSFELD und HUININK (1989), S. 399. Vgl. ferner BlosSFELD et al. (1991), BRÜDERL und KLEIN (1991), NEBENFUHR (1995), S. 209.

334 Vgl. ENGSTLER und LUSCHER (1991). 
ten einer Unterbrechung der Erwerbstätigkeit, und wirken somit in Richtung einer geringeren Kinderzahl. ${ }^{335,336,337}$

Bei vielen der aufgezählten Gründe für einen Rückgang der durchschnittlichen Kinderzahl ist zu bedenken, dass es einerseits einen inhaltlichen Zusammenhang zwischen den verschiedenen Erklärungsansätzen gibt, ${ }^{338}$ und andererseits in vielen Fällen der Zusammenhang zwischen der potenziellen Ursache und dem Rückgang der individuellen Kinderzahl nicht unbedingt einseitig ist: Realistischerweise ist eine Selbstselektion der Betroffenen zu unterstellen. ${ }^{339}$ So ist die Inkaufnahme eines mit dem Erwerb weiterer Qualifikation verbundenen kurzfristigen Verdienstausfalls eher für Leute interessant, die in ihrer bisherigen Lebensplanung keine langfristige Unterbrechung der Erwerbstätigkeit zugunsten der Kindererziehung vorgesehen haben (sie haben eine längere Zeit der Erwerbstätigkeit, in der sich die Investition in das Humankapital amortisieren kann.). ${ }^{340}$ Außerdem sind

${ }^{335}$ Dieser Effekt wird aber wiederum geschmälert durch die Tendenz zur Heirat mit Partnern vergleichbarer oder höherer Bildung, und somit höherem Einkommens: Ein höheres Einkommen des Mannes wirkt (c.p.) steigernd auf die durchschnittliche Kinderzahl einer Ehe (vgl. FLEISHER und RHODES (1979); vgl. auch SCHWARZ (1990a) und WINEGARDEN (1984)). Zu den Lebenseinkommensänderungen von Frauen infolge von Unterbrechungen der Erwerbstätigkeit vgl. GALLER (1988), insbes. S. 101 - 108; bei GALLERs Schätzungen wird deutlich, dass auch kurze Unterbrechungen der Erwerbstätigkeit erhebliche Einkommenswirkungen haben, da sie (auch) das Einkommen nach Wiedereintritt in das Erwerbsleben deutlich reduzieren. GALLER unterscheidet allerdings nicht zwischen direkten Folgen der Erwerbsunterbrechung einerseits, und den möglichen Folgen ,geringerer Berufsorientierung' von Müttern, vernachlässigt mithin eine Selbstselektion.

${ }^{336}$ Eine gewisse Bestătigung der Bedeutung von Opportunitătskosten der Kindererziehung bei der Bestimmung der Kinderzahl mag in dem Hinweis von BUTZ und WARD (1979) auf die Herausbildung einer antizyklischen Fertilität (in den USA) zu sehen sein.

${ }^{337}$ Für Darstellungen zur Änderung des Erwerbsverhaltens von (verheirateten) Frauen seit Anfang der 50'er Jahre vgl. SCHWARZ (1985), SCHWARZ (1993b). ZIMMERMANN (1982, S. 554) kommt bei einer Untersuchung zum folgenden Ergebnis: „Das Ausbildungsniveau der Frau hat ... keinen Einfluß auf die gewünschte Kinderzahl, ist aber mit der tatsächlichen Kinderzahl negativ correliert. Das Ausbildungsniveau des Mannes ist mit der gewunschten Kinderzahl positiv und mit der tatsächlichen Kinderzahl negativ assoziiert". (Bei der Interpretation ist allerdings zu fragen, unter welchen ,gedanklichen Restriktionen' die Befragten die Zahl der ,gewünschten Kinder' ermittelt haben.) Vgl. auch DINKEL (1984b), S. 102 - 106.

${ }^{338}$ Man denke hier nur an die schon erwăhnten Wechselwirkungen zwischen Bildung, Opportunitătskosten, Bildung des Partners, Einkommen, Erwerbsorientierung, Alter bei Eintritt in das Erwerbsleben etc.

Vgl. z.B. ZIMMERMANN (1984), S. 182.

340 Vgl. MINCER und POLACHEK (1974), S. S80. 
höhere Löhne z.T. auch Indikatoren einer besonderen Erwerbs- bzw. Karriereorientierung $-^{341}$ dieselben Leute hätten u.U. auch bei geringerer Entlohnung eine vergleichsweise niedrige Bereitschaft, ihre Erwerbstätigkeit für längere Zeit zugunsten einer Kinderbetreuung einzuschränken. ${ }^{342}$

Eine weitere mögliche Ursache des Rückgangs der Geburtenziffer sind Verbesserungen in Verhütungstechniken sowie eine Verbreitung des Wissens um diese Methoden und eine zunehmende Bereitschaft zu ihrer Anwendung. ${ }^{343}$ Aber auch hierbei ist zwischen Ursache und ,Methode' zu unterscheiden. Sicher hat das Wissen um Verhütungstechniken im betrachteten Zeitraum zugenommen und sich die Zuverlässigkeit der Methoden verbessert. Somit ist die Zahl der Fälle, in denen die individuell zu einem gegebenen Augenblick realisierte Kinderzahl die zu diesem Zeitpunkt, gewünschte' übersteigt, gesunken. Es ist aber fraglich, ob nicht dahinter auch ein Rückgang der durchschnittlich ,gewünschten' Kinderzahl steht - und die verbesserten Verhütungsmethoden eben ,nur' eine Überschreitung der Kinderzahl (wirksamer) verhindern. Die Verhütungsmethoden wären dann insoweit primär ,Mittel zum Zweck', und nicht wesentliche Ursache eines Rückgangs der durchschnittlichen Kinderzahl - ihr Beitrag zur Erklärung dieses Rückgangs mithin vergleichsweise gering. ${ }^{344}$

Ein Rückgang des (verfügbaren) Einkommens der potenziellen Eltern kommt, betrachtet man die Entwicklung des Lebensstandards eines repräsentativen Mitglieds der Aktivgeneration im letzten Jahrhundert, wohl kaum als unmittelbare Ursache eines Rückgangs der Kinderzahl in Frage. Das verfügbare Einkommen eines repräsentativen Haushalts mit gegebener Kinderzahl und gegebener Erwerbsstruktur hat im Zeitablauf zugenommen. Eine eventuelle Wirkung des Einkommensniveaus in Richtung einer niedrigeren durchschnittlichen Kinderzahl kann mithin

${ }^{341}$ Vgl. HÖPFLINGER (1982), S. 25.

${ }^{342}$ Vgl. MROZ (1987), S. 774.

${ }^{343}$ Damit verbunden kann eine allgemein postulierte Verringerung der religiösen Bindung der Bevölkerung sein. Auch hier ist aber die Ursache-Wirkungs-Beziehung nicht eindeutig. (Vgl. HÖPFLINGER (1982), S. 25f., HOLZER und MÖNZ (1996), S. 79, Tab. 7, POHL (1975), S. 61, SCHNEEWIND (1977), S. 126 - 128, SPIEB et al. (1984), S. 160, Tab. 3b.)

${ }^{344}$ Vgl. BECKER (1981), S. 141ff. Ein potenziell relevanter Sachverhalt kann sein, dass die Möglichkeit wirksamer Verhütung zu einer Hinauszögerung der Realisation bis zu einem Augenblick führt, in dem das Kinderhaben biologisch bedingt kaum (bzw. nicht) mehr möglich ist. Mit dem zeitlichen Hinausschieben nehmen die Risiken einer Realisierung der gewünschten Kinderzahl mit dem Effekt zu, dass die realisierte Kinderzahl stärker hinter der gewünschten zurückbleibt. 
nicht über einen Einkommenseffekt vermittelt worden sein, es sei denn man postuliert, Kinder seien für ihre (potenziellen) Eltern absolut inferiore ,Güter'. Eine Erhöhung des Lebensstandards geht aber realiter mit den schon thematisierten Steigerungen des realen Stundenlohnsatzes (nach Steuern i.w.S.) einher, d.h. mit zunehmenden Opportunitätskosten einer Erwerbsunterbrechung (z.B. zugunsten der Kindererziehung). Und diese Opportunitätskosten werden den potenziellen Eltern in einer Gesellschaft, in der der Anteil der kinderlosen (Ehe-)Paare zunimmt, deutlich vor Augen geführt: Bei diesen sind gerade in der Zeit, in der es im Falle der Kindererziehung zu einer Unterbrechung der Erwerbstätigkeit auf Seiten eines Partners kommt, beide regelmäßig erwerbstätig. Insoweit verringert die Entscheidung für das Kinderhaben den Lebensstandard zweifach: Zum einen durch die von Kindern verursachten direkten Kosten, die nicht unmittelbar durch eine Verringerung anderweitiger finanzieller Belastungen (z.B. durch geringere Steuerzahlbeträge, Kindergeld, Erziehungsgeld etc.) ausgeglichen werden, zum anderen durch den mit einer Unterbrechung der Erwerbstätigkeit einhergehenden Verdienstausfall (der infolge von ,Karrierenachteilen' selbst bei relativ kurzer Unterbrechung der Erwerbstätigkeit oder einer mit familiären Verpflichtungen einhergehenden Einschränkung der beruflichen Mobilität über den unmittelbaren finanziellen Verlust des Arbeitseinkommens deutlich hinausgeht). Zwar gibt es die diesbezüglichen finanziellen Nachteile des Kinderhabens (zumindest außerhalb des agrarischen Bereichs) schon lange Zeit ${ }^{345}$ aber die Unterschiede zwischen dem Lebensstandard im Falle von Kinderlosigkeit bei gleichzeitiger Erwerbstätigkeit beider Partner einerseits und dem bei Erwerbsunterbrechung eines Partners zugunsten der Kindererziehung andererseits treten inzwischen eben aufgrund eines höheren Anteils kinderloser Paare deutlicher hervor. ${ }^{346}$ Parallel dazu hat die gesellschaftliche Akzeptanz eines kinderlosen Lebensentwurfs wohl zugenommen, so dass die finanziellen Vorteile von Kinderlosigkeit nicht mehr mit einer gewissen gesellschaftlichen ,Stigmatisierung' ,erkauft' werden müssen. ${ }^{347,348}$

${ }^{345}$ BECKER (1960, S. 175) weist darauf hin, dass schon Mitte des vorigen Jahrhunderts (in den USA) die durchschnittliche Kinderzahl deutlich unter der physisch möglichen gelegen habe. Wenn Kindern ihren Eltern per se Nutzen (,psychic income') stiften, so lasse sich diese Abweichung der realisierten von der ,möglichen' Kinderzahl nur durch Kosten des Kinderhabens erklären. Ein deutlicher Beleg für die von Kindern unweigerlich verursachten hohen Kosten findet sich bei FOGEL und ENGERMAN (1974), die in einer Studie uber die Sklaverei in den USA bezüglich des Wertes von Kindern feststellen: „Net earnings were negative until age eight" (ebd., S. 74f.). Der (barwertmäßige) ,Break-even'-Punkt lag ungefăhr bei einem Alter von 27 Jahren (bei einer durchschnittlichen Lebensdauer von ca. 36 Jahren!) (vgl. ebd., S. 153).

Vgl. KAUFMANN (1990), S. 386.

${ }^{347}$ Das Ausmaß der finanziellen Verschlechterung durch das Ereignis ,Geburt eines Kindes' errechnet BERNTSEN (1992, S. 195f.) mit ca. 15 v.H. - in fast 70 v.H. der Fälle verringert sich das verfugbare Einkommen pro Familienmitglied (auch nach Einbeziehung staatlicher kinder- 
Vielmehr wird auf die Lebensweise kinderloser Paare zunehmend quasi als ,Refe-

bezogener Transfers). Die Interpretation solcher Rechnungen ist allerdings nicht ganz unproblematisch: Zum einen hängen die Werte entscheidend von den den einzelnen Haushaltsmitgliedern zugewiesenen Wohlfahrtsgewichten ab. Diese geben eine ,Äquivalenzskala' der notwendigen Ausgaben pro Familienmitglied zur Erreichung eines vorgegebenen Lebensstandards an. Hierbei tritt das Problem auf, dass in Teilen zur Ermittlung einer solchen Äquivalenzskala auf die Verhältniswerte der Sozialhilferegelsätze für einzelne Haushaltsmitglieder zurückgegriffen wird - die wiederum an Untersuchungen über das faktische Ausgabeverhalten angelehnt sind. Änderungen in dieser Äquivalenzskala können zu erheblichen Verschiebungen in den ausgewiesenen ,(Netto-)Kosten' des Kinderhabens führen. Ferner ist bei ,moralisierenden' Interpretationen einer eventuellen Verschlechterung der Wohlfahrtsposition zu bedenken, dass Teile der Ausgaben zugunsten von Kindern freiwilllig erfolgen. Zusätzlich kann festgehalten werden, dass Teile des Rückgangs des gewichteten Pro-Kopf-Einkommens Folge von Präferenzänderungen sind. Wird unterstellt, dass nicht ausschließlich der Konsum marktmäßig zu erwerbender Güter Nutzen stiftet, so kann ein Rückgang des ,gewichteten' Pro-Kopf-Einkommens infolge der Geburt eines Kindes mit einer Verbesserung der subjektiven ,Zufriedenheit' der Betroffenen einhergehen. Verănderungen des gewichteten Pro-Kopf-Einkommens als ,Änderungen der Wohlfahrtsposition' darzustellen, verträgt sich nur mit einem extrem eingeschränkten Verständnis des Begriffes ,Wohlfahrt'. Dies ändert aber nichts daran, dass sich in den meisten Fällen der (materielle) Lebensstandard eines repräsentativen Haushaltsmitglieds der erhebliche Bedeutung für das Ansehen der betreffenden Person haben kann - durch die Geburt eines Kindes verringert. Für Schätzungen der ,Lebenshaltungsaufwendungen für Kinder' in Deutschland vgl. den gleichnamigen Sammelband des BUNDESMINISTERIUMs FÜR FAMILIE, SENIOREN, FRAUEN UND JUGEND (1995). Für weitere Schätzungen, aber auch Problematisierungen, vgl. BROWNING (1992); fur letztgenanntes auch HENDERSON (1949). Vgl. weiterhin auch CORNELIUS (1988), DEATON und MUELlBauer (1986), DiNKEL (1984b), DORBRITZ und SCHWARZ (1996), EGGEN (1997), EULER (1993), GALLER (1988), GÖRZIG (1977), HAUSER (1995), HOCKAUF-SCHNEIDER (1988), KAPTEYN und PRAAG (1976), KLEIN (1984), Muellbauer (1974), Muellbauer (1977), SeneCa und TAussig (1971). Vgl. auch BUNDESMINISTERIUM FÜR FAMILIE UND SENIOREN (1994), insbes. S. 293f. und WIRTSCHAFTSWOCHE Nr. 31 / 1998 vom 23.07.1998, S. 16 - 21. Einen impliziten Eindruck von den Kosten des Kinderhabens geben Untersuchungen der Caritas über den Anteil einzelner ,Bevölkerungsgruppen' an ihren Klienten: Während nur 0,5 v.H. aller Haushalte in den alten Bundesländern 1989 mehr als 3 Kinder unter 18 Jahren umfassten, waren es unter den KlientenHaushalten der Caritas 4,2 v.H. (vgl. HAUSER und HÓBINGER (1993), S. 104, Tab. III.1.4; vgl. auch ebd., S. 102f., insbes. Tabellen III.1.2 \& III.1.3; weitere Indizien für ein verstärktes Zusammentreffen von ,Armut' und ,Kinderhaben' (insbes. auch bei Alleinerziehenden und in den neuen Bundeslăndern) auch in HANESCH et al. (1994).

Bei einer Befragung kinderloser Frauen zwischen 30 und 39 Jahren im früheren Bundesgebiet über mögliche Gründe gegen die Geburt eines Kindes war der als wichtigster genannte Grund das Fehlen eines Partners, gefolgt von der Unvereinbarkeit mit dem Beruf, den Sorgen um die Zukunft eventueller Kinder, um den Lebensstandard und den ,Lebensgenuss' (vgl. DORBRITZ und SCHWARZ (1996), S. 251, Tab. 18, vgl. auch HOLZER und MÜNZ (1996), S. 86 - 93, JÜRGENS (1977), S. 165f., POHL (1995), STÖTZBACH (1993), S. 156ff. (die beiden letztgenannten für Befragungen in den neuen Bundesländern kurz nach der Wiedervereinigung) und URDZE (1977), insbes. S. 157f.). 
renzfall' hingewiesen. Und da in der gegenwärtigen Ausgestaltung des Rentenversicherungssystems ein höheres Einkommen aus abhängiger Beschäftigung mit dem Bezug höherer Renteneinkünfte im Alter einhergeht, trägt die Rentenversicherung - wiewohl sie zu ihrem Erhalt auch auf die Erziehung einer nachfolgenden Generation angewiesen ist - zu einer vergleichsweise geringen Attraktivität des Kinderhabens bei. Erst durch die Rentenversicherung (in ihrer konkreten Ausgestaltung) verringert Kinderlosigkeit mit einhergehender Erwerbstätigkeit das Risiko von Altersarmut; es kommt mithin zu einer Umkehr der Risikopositionen im Vergleich zu einer, familiären Absicherung' für das Alter.

Da die weitaus meisten Kinder in Ehen geboren werden (bzw. in Partnerschaften, die schon kurz nach der Geburt des Kindes in einer Ehe münden) ${ }^{349}$, mag ein Rückgang des Anteils der Verheirateten an den Mitgliedern der Elterngeneration einen weiteren Grund für eine Verringerung der Kinderzahl darstellen. Gerade hierbei ist aber wiederum das Phänomen der Selbstselektion zu bedenken - bei vergleichbaren Einkommen der beiden Partner gibt es in vielen Fällen nur geringe finanzielle Anreize zum Heiraten (eingeschränkter steuerlicher ,Splittingvorteil' infolge ähnlicher Höhe der Einkommen, geringe Notwendigkeit, durch Heirat einen Partner für den Falle des Todes des anderen Partners finanziell abzusichern etc.). Häufig erfolgt die Heirat erst dann, wenn ein Kinderwunsch besteht ${ }^{350}$ insoweit ist dann die Heirat Folge des (geplanten) Kinderhabens, nicht aber die Kinderlosigkeit Folge des Nicht-Verheiratetseins.

Vergleichbares lässt sich über die Wirkungen zunehmender Scheidungswahrscheinlichkeit sagen: Kinder stellen eine Investition in das ,ehespezifische Humankapital' dar; ${ }^{351}$ die von ihnen verursachten Kosten hängen nur zu geringen Teilen von der Lebensform der Eltern ab, der ,Konsumnutzen' ist aber bei Trennung der Elternteile für einen Partner erheblich eingeschränkt (und die Organisation der Kindererziehung ist für den mit der Kindererziehung ,beauftragten' Elternteil besonders kompliziert). Aus theoretischer Sicht spricht vieles dafür, dass Investitionen in Kinder unterbleiben, wenn die Partner nennenswerte Zweifel am längerfristigen Weiterbestand der Partnerschaft haben - wobei unterlassene Investitionen in das ehespezifische Humankapital selber auch als Grund für eine er-

${ }^{349}$ SCHWARZ (1993a, S. 75) verweist darauf, dass mehr als ein Drittel der nichtehelich geborenen Kinder „durch nachträgliche Eheschließung der Eltern legitimiert wird“. Vgl. auch SCHWARZ (1995).

${ }^{350}$ Für eine Zunahme der Bedeutung des Heiratsanlasses ,(geplante) Schwangerschaft' vgl. POHL (1985), S. $95 f$.

${ }^{351}$ Vgl. BECKER (1981), S. 329ff. 
höhte Wahrscheinlichkeit einer Trennung der Partner gelten mögen. Insoweit ist eine Erhöhung des Anteils der scheiternden Ehen an allen geschlossenen Ehen (sowie eine davon möglicherweise ausgehende ,psychologische' Wirkung auf die subjektive Wahrnehmung des Risikos des Scheiterns der eigenen Partnerschaft) zugleich mögliche Ursache und Folge vergleichsweise niedriger Kinderzahlen. ${ }^{352,353,354}$

$\mathrm{Zu}$ nennen ist abschließend die zunehmende Verstädterung, und die mit ihr einhergehende Änderung der Beschäftigungs- und Sozialstruktur: In einem agrarischen Umfeld war eine innerfamiliäre Alterssicherung durch das ,Kinderhaben' möglich. Die Kinder (bzw. der Älteste) erhielt im Tausch für die Zusage einer Versorgung der alten Eltern den Hof (bzw. die Möglichkeit seiner Nutzung). Ein entsprechendes Tauschpotenzial besteht für Industriearbeiter etc. nicht; der Übergang zu städtischen Lebensformen entwertet vielmehr selbst das noch vorhandene Tauschpotenzial. Das Aufziehen von Kindern hat aus individueller Sicht in einem industriell geprägten Umfeld eine geringere Bedeutung für die Sicherung des Lebensstandards im Alter. ${ }^{355}$ Ferner ist es im agrarischen Bereich eher möglich, Kindererziehung mit nur geringfügig eingeschränkter Erwerbstätigkeit zu verbinden, was niedrigere Opportunitätskosten von Kindern für Landwirte als für andere Bevölkerungsgruppen bedingt. ${ }^{356}$ All dieses legt nahe, dass die abnehmende Bedeutung des ländlichen Raumes mit einer verringerten durchschnittlichen Kinderzahl einhergeht.

Es ist aber wiederum die Bedeutung der Selbstselektion zu beachten: $\mathrm{Da}$, wo eine Wahlmöglichkeit besteht, ist das Leben in der Stadt für Kinderlose sicher ,inter-

${ }^{352}$ Vgl. BECKER (1981), S. 329ff.

${ }^{353}$ Kinder stellen auch eine Belastung für das Eingehen einer neuen Partnerschaft nach dem Scheitern einer Beziehung dar. So errechnet SCHWARZ (1984, S. 14 - 17) eine Wiederverheiratungswahrscheinlichkeit für kinderlos Geschiedene von uber 90 v.H., während der entsprechende Wert für Kinderhabende bei 40 v.H. liegt. Vgl. auch GoSTOMSKI (1998).

${ }^{354} \mathrm{Vgl}$. auch die schon (unter 2.5.3) thematisierte Untersuchung von EHRLICH und ZHONG (1998).

${ }^{355}$ Ferner wird die Möglichkeit der Kinder verringert, parallel zu ihrer Erwerbstătigkeit die Eltern zu ,betreuen', wobei diese sich wiederum u.U. bei der Betreuung ihrer Enkel ,nützlich' machen können.

${ }^{356}$ Hinzu kommt eine gute Möglichkeit, eine partielle ,Refinanzierung' der Kinder in Form von Mithilfe auf dem Hof zu betreiben. Eine vergleichbare Möglichkeit besteht in Arbeiter- und Angestelltenhaushalten kaum; hier ist ausschließlich eine Mithilfe der Kinder bei der Produktion von ,Haushaltsgütern' möglich. 
essanter' als für Eltern - und aufgrund der höheren Kosten für Wohnung etc. auch eher für doppelverdienende Paare bzw. Alleinlebende finanzierbar.

Die Vielschichtigkeit der potenziellen Gründe für einen Rückgang der durchschnittlichen Kinderzahl hat zur Folge, dass eine Politik, die die Bildung von Humankapital fördern will, an unterschiedlichen Punkten ansetzen muss - die Rentenversicherung ist diesbezüglich nur ein Ansatzpunkt unter Vielen, wenn auch wegen des großen betroffenen Finanzvolumens ein bedeutsamer. Ein weiterer, in Teilen auch der politischen Gestaltung zugänglicher, Ansatzpunkt ist eine Senkung der Opportunitätskosten der Kindererziehung bei Verzicht auf bzw. längerer Unterbrechung der Erwerbstätigkeit: Da sich die Rentenhöhe bei stärkerer ,Kinderorientierung' des Rentenversicherungssystems weniger an der Höhe der Beitragszahlungen zur Rentenversicherung orientiert, bedeutet ein ,Verzicht' auf Einzahlungen mithin eine geringere Reduktion der späteren Rente (ein Argument, das allerdings auch für einen Verzicht auf Erwerbstätigkeit aus anderen Gründen als den der Kindererziehung gilt), und das Kinderhaben erhöht den späteren Rentenanspruch stärker. Längerfristig mag auf diese Art und Weise die Bereitschaft zur Unterbrechung bzw. Beendigung der Erwerbstätigkeit zugunsten der Kinderbetreuung gesteigert werden - vermutlich sollte man aber bezüglich der Intensität einer solchen Reaktion keine übertriebenen Erwartungen haben. Auch eine verbesserte Anerkennung von Erziehungsleistungen im Rahmen der GRV kann nicht verhindern, dass Kinder Geld kosten, und die Unterbrechung der Erwerbstätigkeit regelmäßig mit einem Verlust an Einkommen (und späterer Rente) verbunden ist. Ursächlich dafür ist eben nicht allein die Rentenversicherung (bzw. deren Ausgestaltung), so dass deren Aufgabe nicht die generelle Behebung dieses möglichen Mangels sein kann, sondern ,nur' eine Korrektur eventueller Verzerrungen, die ursächlich in der Ausgestaltung der GRV begründet liegen. In Anbetracht der Vielzahl der Gründe für einen Rückgang der durchschnittlichen Kinderzahl, und insbesondere auch der ,Normalität' von Kinderlosigkeit bzw. Ein-Kind-Familien etc. erscheint es zweifelhaft, ob auch volumenmäßig erhebliche Stärkungen der Kindererziehung im Rahmen des Rentenrechts den schon lange bestehenden Trend zu einer nicht ,populationserhaltenden' durchschnittlichen Kinderzahl umkehren können. Längerfristig mag sich das gesellschaftliche ,Meinungsklima' infolge verbesserter finanzieller Ausstattung von Familien zu Gunsten der Kindererziehung jedoch ändern, mit entsprechenden Auswirkungen auf die durchschnittliche Kinderzahl - aber solche ,Meinungsumschwünge' brauchen Zeit.

Insbesondere ist bei den Wirkungen einer verstärkten Kinderberücksichtigung im Rahmen der Rentenversicherung zu bedenken, dass eventuelle positive (Lebens-)Einkommenseffekte der veränderten Ausgestaltung zugunsten Kindererziehender nicht allein in Richtung einer erhöhten Kinderzahl hin Anreiz geben, sondern auch in Richtung einer Substitution von Kinderquantität durch Kinderqualität, also hin 
zu einer Erhöhung der Ausgaben pro Kind. Dieser Effekt muss für die ,Überlebensfähigkeit' der Rentenversicherung in der Zukunft keinesfalls negativ sein, da, wie im Verlauf der Arbeit schon mehrfach betont, die Summe der (realen) rentenversicherungspflichtigen Einkommen der Mitglieder der Nachfolgegeneration und nicht deren Umfang für die Befriedigung von Rentenansprüchen entscheidend ist; ob zur Zeit Investitionen in die Quantität oder die Qualität der nachfolgenden Generation für den Erhalt der Rentenversicherung vorteilhafter sind, kann hier nicht entschieden werden.

Um eine deutlichere und schnellere Wirkung zu zeigen, bedarf eine stärkere Kinderorientierung im Rahmen der Gesetzlichen Rentenversicherung der ,Flankierung' durch weitere Maßnahmen: Zu nennen wären hier insbesondere die Ausgestaltung des Steuerrechts einerseits und der Staatsausgaben andererseits. Eine mögliche derartige Maßnahme könnte eine Verbesserung der Vereinbarkeit von Familie und Beruf - gerade auf Seiten der Frauen - sein. ${ }^{357} \mathrm{Ob}$ allerdings eine ,kinderfreundliche' Ausgestaltung z.B. durch Anlehnung an das Beispiel Schwedens erstrebenswert ist, ${ }^{358}$ sei dahingestellt: Die ,intensive' Versorgung der Bevöl-

${ }^{357}$ Für Umfrageergebnisse, nach denen die absolute Mehrheit der Frauen eine Kombination von Teilzeit- bzw. Vollzeiterwerbstätigkeit als ,ideale Kombination von Beruf und Familie' ansieht, vgl. DORBRITZ und FleISCHHACKER (1995), S. 179, Tab. 3. Die Bevorzugung des Lebensmodells ,Erwerbstătigkeit mit Kind' ist bei den befragten Frauen in der ehemaligen DDR noch wesentlich höher als in Westdeutschland. Bei solchen Befragungen ist allerdings zu berücksichtigen, dass die gegebenen Antworten vermutlich nicht von den aktuellen Lebensumständen unbeeinflusst geblieben sind (So z.B. von der Tatsache, dass bei erhöhtem Risiko der Arbeitslosigkeit eine Erwerbstätigkeit beider Ehepartner sinnvoll ist, um im Falle der Arbeitslosigkeit des einen Partners vom Einkommen des anderen (zuzüglich des Arbeitslosengeldes) vergleichsweise, gut' leben zu können. Ferner prägt die erwerbsorientierte Ausgestaltung in der ehemaligen DDR sicher noch die Einstellungen der Befragten. Und aus Sicht der Befragten kann ,ideal' immer nur als ,ideal unter den gegenwärtigen Bedingungen' verstanden werden, also nicht als globales Optimum, sondern als lokales in einem restringierten ,Entscheidungsbereich'.).

Das ,schwedische Modell' ist gekennzeichnet durch eine hohe steuerliche Belastung der Einkommen bei gleichzeitig starker Subventionierung von Kindertagesstätten. Die besondere Logik des Systems wird von LINDBECK (1997) wie folgt charakterisiert: „A combined result of the tax and benefit system in Sweden is that ,the care of things,' such as maintenance of property and durable consumer goods has shifted from the market to the household sector (because of wide marginal tax wedges), while ,the care of individuals' has shifted from the family to the government sector (because of high subsidies to public-sector services, e.g. for children ...). ... [T] he Swedish welfare state has .., to a broad extent, socialized the provision of personal services to individuals and families“ (ebd., S. 1300f.). Hierzu passt auch die Pointierung von ROSEN (1996): „In Sweden a large fraction of women take care of the children of women who work in the public sector to care for the parents of the women who are looking after their children. If Swedish women take care of each other's parents in exchange for taking care of each other's children, how much additional output comes of it?' LINDBECK (1997) sieht als einen Vorteil des ,schwedischen Modells' - mit Blick auf das für die vorliegende Arbeit interessie- 
kerung durch den Staat - z.B. in Form eines ,gut ausgebauten' Systems außerhäuslicher Betreuungseinrichtungen für Kinder - muss unter Hinnahme (c.p.) erhöhter Durchschnitts- und Grenzsteuersätze ,gegenfinanziert' werden. ${ }^{359}$ Entsprechendes gilt auch für das nahe am vorherigen Nettoarbeitsentgelt des jeweiligen Elternteils angesiedelte staatliche ,Ersatzeinkommen'. Über die hohe Belastung der Arbeitseinkommen mutiert das staatliche Angebot einer Vereinbarkeit von Familie und Erwerbstätigkeit de facto zu einem Zwang, nach Ablauf der ,Kindererziehungszeit' zu einer Erwerbstätigkeit beider Elternteile überzugehen, und ist mithin kaum als familienfreundlich zu charakterisieren.

Bei Berücksichtigung der vielen Indizien, die eine kurzfristige, deutliche Ausweitung der durchschnittlichen Kinderzahl (auch) im Falle einer gesteigerten Kinderberücksichtigung im Rahmen der GRV unwahrscheinlich erscheinen lassen, ist ein Kinderleistungsausgleich im Rahmen der GRV vor allem im Sinne einer Auf-

rende Gebiet - eine vergleichsweise geringe Armut von Kindern an. Allgemeiner kann gesagt werden, dass das ,Risiko', das Kinder für den Lebensstandard ihrer Eltern darstellen, in Schweden niedrig ist. Mit der (u.a.) zur Finanzierung staatlicher Familienförderung verbundenen steuerlichen Belastung von (Arbeits-)Einkommen gehen aber (u.a.) geringe Anreize zur Akkumulation von Humankapital einher, so dass hier wiederum der Staat ,gezwungen' ist, subventionierend ,einzugreifen'; auf weitere problematische Wirkungen hoher marginaler und durchschnittlicher Steuersătze (Es gibt in Schweden kein Ehegattensplitting, so dass die Progression der Einkommensteuer einen Anreiz zur Erwerbstätigkeit beider Ehepartner setzt!) ist hier nicht weiter einzugehen. Für einen Verweis auf den hier charakterisierten, schwedischen Ansatz' (bzw. seine Ausprägung in der ehemaligen DDR) vgl. SCHWARZ (1992b), S. 199 - der allerdings auch auf die ,enormen Kosten' einer derartigen Ausgestaltung hinweist.

Das gerade Gesagte gilt nur für den Fall einer staatlichen Bereitstellung von Kinderbetreuungseinrichtungen - und zwar zu einem nicht die (variablen und sprungfixen) Kosten der Einrichtung deckenden Preis. Da es aber schon heute kein grundsätzliches Verbot privater Kinderbetreuung gibt, deuten Klagen uber eine ,Unterversorgung' (West-)Deutschlands mit Stätten der Ganztagskinderbetreuung darauf hin, dass zu kostendeckenden Preisen, also ohne staatliche Subventionierung, wohl kaum zusätzlicher Betreuungsbedarf existiert. Forderungen nach einem Ausbau der Kinderbetreuung laufen also unmittelbar auf Forderungen nach Bereitstellung subventionierter Kinderbetreuung hinaus (vgl. LAMPERT (1993), S. 355). Zur Preiselastizität der Nachfrage nach ,professioneller' Kinderbetreuung (im Gegensatz zur Betreuung durch Verwandte, ăltere Geschwister etc.) und zur Abhängigkeit der Entscheidung zur Aufnahme bezahlter Arbeit von den Kosten der Kinderbetreuung vgl. BLAU und ROBINS (1988), HECKMAN (1974), KLERMAN und LEIBOWITZ (1990), RoBINS und SPIEgELMAN (1978). Die Vorzeichen der empirisch ermittelten Elastizitäten haben in diesen Untersuchungen fur die USA jeweils das erwartete (negative) Vorzeichen - und sind in den meisten Fälen auch signifikant. Zu vergleichbaren Ergebnissen kommt auch CONNELLY (1992). Letztere verweist darauf, dass die geringere Erwerbspartizipation von Frauen mit jungen Kindern in wesentlichen Teilen mit den vergleichsweise hohen Kosten einer Betreuung dieser Kinder zu erklären ist (vgl. ebd., S. 89). Für eine Untersuchung Kanadas (ohne überraschende empirische Ergebnisse) vgl. CLEVELAND et al. (1996). 
teilung der finanziellen Lasten der Kindererziehung auf diejenigen, die durch die Systematik der Rentenversicherung von diesen Ausgaben bzw. den durch sie induzierten Ergebnissen finanziell profitieren, zu begründen. Gerade eine finanzielle Entlastung der Eltern in der Zeit der Kindererziehung ist auch in dieser Hinsicht wichtig $-^{360}$ wobei die Probleme einer Umstellung des Rentenversicherungssystems vom bestehenden System hin zu einem sich davon fundamental unterscheidenden System erheblich sind.

\subsection{Kann es eine politische Mehrheit für eine kinderfreundliche Reform der Rentenversicherung geben?}

Gerade wenn eine Umstellung der Rentenversicherung hin zu einer stärker generative Leistungen berücksichtigenden Ausgestaltung zwar sinnvoll erscheint, aber mit erheblichen Problemen verbunden ist, so dass eine solche Reform kein ,Selbstgänger' zu werden verspricht, ist nach ihren Chancen im politischen Prozess zu fragen. Ob die Politik zu einer ,kinderfreundlichen' Umgestaltung der Rentenversicherung die ,Kraft' hat, erscheint zweifelhaft. In den letzten Jahrzehnten waren Änderungen, die zu einer Stärkung der Anerkennung der Bedeutung des Kinderhabens für den Fortbestand (und die Rendite) der Rentenversicherung führten, vor allem Folge entsprechender Forderungen des Bundesverfassungsgerichts. Die Politik präsentierte sich beim Ausbau der Kinderberücksichtigung im Rahmen der Rentenversicherung eher als getriebene denn als treibende Kraft; und selbst bei diesen Umgestaltungen wurden die entstehenden Kosten als ,versicherungsfremde Leistungen' charakterisiert und dienten der Begründung eines gesteigerten Bundeszuschusses. Eine Einschätzung, die Berücksichtigung generativer Leistungen sei im Rahmen einer umlagefinanzierten Rentenversicherung systemimmanent, ist weiterhin kaum zu vernehmen.

Politökonomisch betrachtet sind aber nicht unbedingt, bessere Einsichten' der Politiker ursächlich für deren mögliche Eingriffe, sondern eine, gesellschaftliche Erwartungshaltung' müsste ihnen im Sinne einer Erhöhung ihrer Chancen auf (Wieder-)Wahl derartige Reformen lohnend erscheinen lassen. Es ist mithin zu überlegen, ob Reformen hin zu einer ,kinderfreundlicheren' Ausgestaltung der Rentenversicherung bei der (wahlberechtigten) Bevölkerung populär sein können,

${ }^{360}$ Dies gilt umso mehr, als durch eine finanzielle Entlastung der Eltern in der Kindererziehungsphase eventuelle Liquiditătsprobleme der Eltern, die deren Entscheidung über die Höhe der Investition in Kinderqualităt beeinflussen können, vermindert werden (vgl. BECKER (1989), BECKER und TOMES (1979), BOWLES (1972)). Das hier charakterisierte Problem ist sicher in den USA - auf die sich die aufgefuhrten Untersuchungen beziehen - mit einer niedrigeren unmittelbaren staatlichen Finanzierung von Bildungseinrichtungen größer als in Deutschland. 
ob ihre Propagierung mithin geeignet erscheint, die Wahlchancen von Parteien oder Politikern zu verbessern.

Ein erster Hinderungsgrund für eine Wählerwirksamkeit entsprechender Reformen der GRV ist die schon heute bestehende schwere Verständlichkeit des Rentenrechts. Diese hemmt eine einprägsame und nachvollziehbare Darstellung von Änderungsvorschlägen. Dementsprechend verspricht die Führung eines inhaltlichen Wahlkampfes über das Thema Rente - sowohl für die Wähler, als auch (dementsprechend) für die Politiker - wenig attraktiv zu sein. Obendrein dürften Diskussionen um Änderungen der Ausgestaltung der Rentenversicherung recht schnell zu einer ,Verängstigung' weiter Teile der Bevölkerung führen, wenn nicht von vornherein gesagt wird, dass es - zumindest unter den Rentnern und einer großen Zahl von Leuten im ,rentennahen Alter' - keine ,Verlierer' einer etwaigen Rentenreform geben wird. ${ }^{361}$ Eine solche Einschränkung der Wirkungen einer eventuellen Rentenreform aber reduziert den Handlungsspielraum. ${ }^{362}$ Die $\mathrm{Zu}-$ nahme des Anteils der (wahlberechtigten) Bevölkerung, der entweder bereits eine Rente bezieht, oder aber nur noch wenige Jahre bis zur Verrentung vor sich hat, erschwert eine Reform weiter: Immer mehr Wähler sind bei eventuellen Senkungen des Rentenniveaus besonders ,schnell' und ,intensiv' betroffen, ohne von den eventuellen Vorteilen der Reform, die sich als Entlastung der Beitragszahler materialisieren, profitieren zu können.

Entsprechendes gilt auch für Reformen, die zu einer Stärkung der Kinderberücksichtigung führen. Wie in der Arbeit mehrfach betont, führen solche Reformen (zumindest für eine Übergangsphase) zu einer Senkung der Rendite von ,Beitragszahlungen'. Auch wenn die Abschätzung dieser Rendite kompliziert ist, erleichtert dies eine Reform nicht, besteht erst einmal ein Meinungsklima, jeder Umbau

${ }^{361}$ Die wenigsten Änderungen in der Rentenversicherung dürften diesem Kriterium genügt haben. Realiter mag es also ausreichen, wenn man denjenigen, die die aktive Wählerschaft stellen, einen weitgehenden Schutz vor Verschlechterungen ihrer Rentenposition zusichert; außerdem mag zwischen durch Änderungen der Rentenversicherung induzierten Senkungen der Rentenhöhe (im Vergleich zur Situation ohne die Änderungen) einerseits und durch sie induzierten Änderungen der ,Rendite' der Beitragszahlungen zu unterscheiden sein, wobei die erstgenannten Änderungen leichter einsichtig und möglicherweise auch deshalb politisch sensibler sind.

${ }^{362}$ Die Diskussion um das ,Rentenreformgesetz 1999' hat gezeigt, dass selbst die Zusage, dass es infolge der Änderungen im Rentensystem zu keinen Senkungen des absoluten Rentenniveaus eines einzigen Rentenbeziehers kommen wird, zusammen mit weitestgehender Übereinstimmung der wissenschaftlichen Berater, die vorgeschlagene Rentenreform fuhre tendenziell in die richtige Richtung, nicht ausreicht, um eine weitgehende Akzeptanz der Reform bei den Alten herbeizufuhren. 
des Sozialsystems diene automatisch seinem Abbau. Und in der Vergangenheit ist der Bevölkerung gerade von politischer Seite wiederholt erklärt worden, die entscheidende Größe zur Bestimmung der Rentenhöhe sei in den vorangegangenen Beitragszahlungen zur GRV zu sehen; die Anerkennung von Kindererziehungsleistungen im Rahmen der Rentenversicherung habe eher den Charakter ,schmükkenden Beiwerks'. Ist die Bevölkerung erst einmal von einer derartigen Sichtweise so überzeugt, dass sie sie für, natürlich' hält, dann begünstigt dies einen Strukturkonservativismus, der Änderungen entgegen steht. Es besteht damit die Möglichkeit, dass die Reform auch von weiten Teilen der Bevölkerung abgelehnt wird, die von einer Stärkung der Kinderberücksichtigung profitieren können: $\mathrm{Zu}-$ erst zu nennen wären hier Versicherte mit einer überdurchschnittlichen Investition in das Humankapital der nachfolgenden Generation; auf Grund einer Minderung der Diskriminierung der Bildung von Humankapital kann aber auf längere Sicht auch die Rendite des Rentenversicherungssystems gesteigert werden, so dass auch die Gruppe ,jüngere Versicherte' allgemein zu den potenziellen Gewinnern eines Umbaus gehört. Die Jungen könnten am ehesten von den Verantwortlichen Reformen einfordern: Gerade unter ihnen sind Zweifel an der ,Sicherheit' der (oder: ihrer) Rente verbreitet, und mithin Aufgeschlossenheit gegenüber Reformansätzen zu erwarten. Die Jungen sind es aber auch, denen es möglich ist, auf veränderte Anreizkonstellationen im Rentenversicherungssystem infolge einer verstärkten Berücksichtigung generativer Leistungen durch Verhaltensänderungen zu reagieren. Sie sind diesbezüglich also in einer besseren Ausgangsposition als ältere Versicherte. Letzteren ist eine Substitution von Beitragszahlungen durch Kindererziehungsleistungen nicht mehr möglich, was für sie die Gefahr einer Verschlechterung ihrer jeweiligen Rentenposition infolge einer Reform erhöht, und somit eine Ablehnung der Reform begünstigt.

Ob Reformansätze ,mehrheitsfähig' sind, ist nicht ohne weiteres zu entscheiden: Insbesondere ist zu fragen, ob und in welcher Intensität die Wählerschaft auf (vermeintliche) Änderungen der Höhe und ,Sicherheit' ihrer Rente durch ,Androhung' einer veränderten Stimmabgabe bei Wahlen reagiert, ${ }^{363}$ oder ob sie im Sinne einer längerfristigen Sicherung des Rentensystems gewisse Verschlechterungen der eigenen Rentenposition hinnimmt.

Die bisherige Argumentation kann ausgeweitet werden, indem unterstellt wird, nicht der Vorteil des einzelnen Mitglieds der Rentenversicherung sei für seine Zustimmung zu einer eventuellen Umstrukturierung relevant, sondern der kumulierte

${ }^{363}$ In seiner einfachsten Form läuft die Argumentation auf die Feststellung hinaus, der ,Medianwähler' habe (da er von den höheren Leistungen im Alter voll - und schon recht bald - profitiere, die Lasten in Form vergleichsweise hoher Beiträge aber nur für eine recht kurze Zeit zu tragen habe) ein Interesse an einer Ausweitung der Rentenleistungen (vgl. PETERSEN (1984)). 
Vorteil der Mitglieder seiner Familie (Ehepartner, Kinder etc.). ${ }^{364}$ Allerdings ist bei der Nutzung der diesbezüglichen Untersuchungen BREYERs und SCHULENBURGs (insbes. (1987a) und (1989)) Vorsicht geboten: Die von Ihnen für viele verschiedene Parameterkonstellationen ausgewiesene weitgehende Ablehnung einer umlagefinanzierten Rentenversicherung durch die Mehrheit der Stimmberechtigten liegt partiell in kritischen Annahmen begründet: Zum einen gibt es in diesen Untersuchungen keine Unsicherheit (z.B. über den Todeszeitpunkt), zum anderen wird - dementsprechend - keine Sicherungsfunktion der Rentenversicherung für Hinterbliebene abgebildet. Gerade diese Funktion der Rentenversicherung kommt aber realiter zum Tragen, wenn besonders 'Sicherungsbedüftige' (also gerade kleine Kinder, die von einem Elternteil betreut werden, so dass nur geringes Einkommen jenseits des durch den Tod ausfallenden Einkommens des Allein- / Hauptverdieners zur Verfügung steht) vorhanden sind. Insoweit hat die GRV in der Realität auch für Familien gewisse Vorteile. Bedenkt man dann noch mögliche Probleme der intrafamiliären Einkommensübertragung (insbesondere von den Kindern an die Eltern - wenn letztere keine Erbschaft hinterlassen wollen, die gemindert werden kann, um so einen ,Zwangstransfer' zu gewährleisten), sowie Probleme des Einkommenstransfers von der Zukunft in die Gegenwart ohne ausreichende Sicherheiten, so erscheint die Mehrheit einer Ablehnung des bestehenden Systems nicht so sicher, wie es die Werte in den Untersuchungen anzudeuten scheinen. Insbesondere ist die Abschaffung jedweden Alterssicherungssystems, d.h. die Reduktion auf familiäre Alterssicherung, eine Extremposition, die unter realitätsnäheren Annahmen sicher nicht ernsthaft zur Debatte steht.

Eine ,kinderorientierte' Ausgestaltung des Rentenversicherungssystems stellt eine Alternative dar, die die Vorteile einer breiten Tragung eines Alterssicherungssystems nutzt, ohne die Bedeutung von Kindern für die Alterssicherung bei der Bestimmung des Rentenanspruchs auszublenden. Neben Leuten, die von einer solchen Reform eindeutig profitieren würden, und denjenigen, die durch sie erhebliche finanzielle Verluste erleiden würden, existiert eine Gruppe von Leuten, deren (erwartete) Vor- oder Nachteile nicht allzu groß sind. Während die Zustimmung resp. Ablehnung einer Umgestaltung durch die Mitglieder der beiden erstgenannten Gruppen ziemlich sicher sein dürfte, sind die Mitglieder der ,Mittelgruppe' in ihrer Entscheidung offen: Zwar ist ihre Akzeptanz der Reform nicht als gesichert anzusehen, sie können aber möglicherweise durch Information über die

${ }^{364}$ Für die Nutzung eines solchen Ansatzes fur den Fall einer Abstimmung uber den Fortbestand bzw. die Ausgestaltung der Rentenversicherung vgl. BREYER und SCHULENBURG (1989); vgl. ferner BREYER (1992). Fur die Betonung der Familie als relevante ,Instanz', deren Vorteil aus einem jeweiligen Abstimmungsgegenstand miteinander verglichen wird, siehe BREYER und SCHULENBURG (1987a); für entsprechende Überlegungen zur Krankenversicherung vgl. BREYER und SCHULENBURG (1987b). 
Sinnhaftigkeit einer Umgestaltung (und einige - nicht allzu ausgabewirksame Zusagen bezüglich einer maximalen ,Schlechterstellung' bei erfolgender Umgestaltung), gewonnen' werden.

Die Ablehnung einer Reform dürfte in vielen Fällen mithin nicht so stark sein, dass entschlossene Politiker sie nicht u.U. überwinden können. Dies gilt insbesondere dann, wenn eine in nennenswertem Ausmaß parteigebundene (resp. ,parteitreue') Wählerschaft unterstellt wird, die einem Kandidaten ihrer grundsätzlichen politischen Richtung nicht automatisch die Zustimmung verweigert, wenn das von ihm unterstützte Programm ihr auch nur leichte finanzielle Nachteile bringt. Allerdings muss ein auf Vorsicht bzw. (Wieder-)Wahl bedachter Politiker darauf bedacht sein, nicht allzu große Teile seiner Stammwähler vergleichsweise stark zu belasten ${ }^{365} \mathrm{Ob}$ eine stärkere Berücksichtigung der Erziehungsleistung im Rahmen der Rentenversicherung gesellschaftlich mehrheitsfähig ist, erscheint unsicher - dass sie es nicht ist, wenn sich diejenigen, die von der Sinnhaftigkeit eines solchen Ansatzes überzeugt sind, nicht in der Öffentlichkeit engagiert für eine Reform aussprechen, kann als sicher gelten.

${ }^{365}$ Diese Argumentation hat eine Năhe zur Aussage BREYERs und SCHULENBURGs, die feststellen: „... die in jungster Zeit gemachten Vorschlăge, die Beitragssătze (oder die Leistungen) der Rentenversicherung von der Kinderzahl abhängig zu machen ... und daher das Ausmaß impliziter Umverteilung von Familien mit vielen zu solchen mit wenigen Kindern zu verringern, [scheinen] ein vernüntiger Weg zu sein, die Überlebenschancen des Rentensystems in der Demokratie zu verbessern" (dies. (1989), S. 137). Die beiden Autoren beziehen die ,Überlebenschancen' des Systems auf die Frage, ob eine Mehrheit der Bevőlkerung fur die Beibehaltung des Rentenversicherungssystems stimmt, oder nicht. Im Rahmen ihrer Argumentation bedarf es zur Sicherung einer Zustimmung der Mehrheit der Bevolkerung - tendenziell infolge des Übergewichts Kinderhabender an der Wahlbevölkerung - kinderfreundlicher Ausgestaltungsformen der Rentenversicherung. 


\section{Anhänge}

\subsection{Anhang 1: Formale Darstellung der Kinderberüicksichtigung während der Beitragszeit bei einer Rentenversicherung nach Version 2}

Der ,rentenmäßige externe Effekt' eines weiteren Kindes beträgt bei einem Rentenbeitrag von $b_{t+1}$

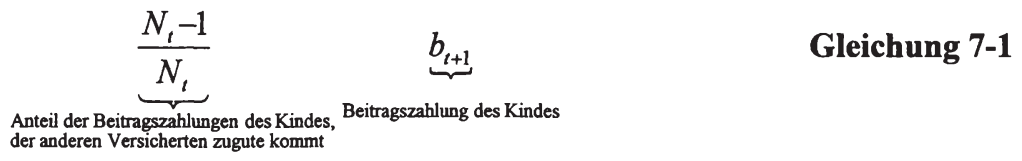

Dieser Wert wird mit der ,Verzinsungsrate' der Beitragszahlungen in der GRV

$$
\frac{N_{t+1}}{N_{t}}
$$

abdiskontiert.

Da eine intertemporal konstante Rentenzahlung ( $r$ ) unterstellt wird, beträgt die (spätere) Beitragszahlung eines repräsentativen Kindes

$$
b_{t+1}=r \cdot \frac{N_{t}}{N_{t+1}} .
$$

\section{Gleichung 7-2}

Aus den getroffenen Annahmen lässt sich der Barwert des externen Effektes eines Kindes innerhalb der Rentenversicherung berechnen als

$$
\frac{N_{t}-1}{N_{t}} \cdot r \cdot \frac{N_{t}}{N_{t+1}} \cdot \frac{N_{t}}{N_{t+1}}=\frac{N_{t}-1}{N_{t+1}} \cdot r \cdot \frac{N_{t}}{N_{t+1}} .
$$

Aus der Forderung, dass die Beitragssumme durch die Kinderberücksichtigung nicht verändert werden soll, und die rentenmäßige Berücksichtigung aller Kinder 
einheitlich sein soll (sie werden ja später auch einen einheitlichen Beitrag zur RV leisten), ergibt sich die Forderung

$$
N_{t} \cdot b_{t}=\sum_{n}\left\{N_{t, n} \cdot\left(b_{t}^{0}-n \cdot \frac{N_{t}-1}{N_{t+1}} \cdot r \cdot \frac{N_{t}}{N_{t+1}}\right)\right\}
$$

(mit $b_{t}^{0}$ : Rentenversicherungsbeitrag eines Beitragszahlers mit 0 Kindern, $N_{t, n}$ : Zahl der Beitragszahler in $t$ mit $n\left(n \in N^{0}\right)$ Kindern).

Diese Gleichung lässt sich umformen zu

$$
N_{t} \cdot b_{t} \stackrel{!}{=} b_{t}^{0} \cdot \sum_{n} N_{t, n}-r \frac{N_{t}-1}{N_{t+1}} \cdot \frac{N_{t}}{N_{t+1}} \cdot \sum_{n}\left\{N_{t, n} \cdot n\right\}
$$

und wegen $\sum_{n} N_{t, n}=N_{t}$ und $\sum_{n}\left\{N_{t, n} \cdot n\right\}=N_{t+1}$ sowie $b_{t} \cdot N_{t}=r \cdot N_{t-1} \mathrm{zu}$

$$
r \cdot N_{t-1} \stackrel{!}{=} b_{t}^{0} \cdot N_{t}-r \cdot \frac{N_{t}}{N_{t+1}} \cdot\left(N_{t}-1\right)
$$

Hieraus lässt sich $b_{t}^{0}$ berechnen als

$$
b_{t}^{0}=r \cdot \frac{N_{t-1}}{N_{t}}+r \cdot \frac{\left(N_{t}-1\right)}{N_{t+1}} .
$$

\section{Gleichung 7-7}

Für $b_{t}^{n}$ (Rentenversicherungsbeitrag eines Beitragszahlers mit $n$ Kindern) folgt hieraus:

$$
b_{t}^{n}=r \cdot \frac{N_{t-1}}{N_{t}}+r \cdot \frac{\left(N_{t}-1\right)}{N_{t+1}}-n \cdot \frac{\left(N_{t}-1\right)}{N_{t+1}} \cdot \frac{N_{t}}{N_{t+1}} \cdot r=r \cdot \frac{N_{t-1}}{N_{t}}+r \cdot \frac{\left(N_{t}-1\right)}{N_{t+1}} \cdot\left(1-n \cdot \frac{N_{t}}{N_{t+1}}\right) .
$$

\section{Gleichung 7-8}




\subsection{Anhang 2: Umformung von Version 1 zu Version 2 unter einfachen Bedingungen}

Im Folgenden soll die systematische Übereinstimmung der vorgestellten Kinderberücksichtigungen in den Versionen 1 und 2 für den Fall einer in allen relevanten Perioden sich mit übereinstimmender Nettowachstumsrate ändernden Generationenstärke verdeutlicht werden.

Aus der formalen Darstellung der Kinderberücksichtigung in Version 1 (vgl. Anhang 1 (Abschnitt 7.1) ist als Formel für den Absolutbeitrag eines Versicherten mit $n\left(n \in N^{0}\right)$ Kindern in Periode $t$ bekannt:

$b_{t}^{n}=r \cdot \frac{N_{t-1}}{N_{t}}+r \cdot \frac{\left(N_{t}-1\right)}{N_{t+1}} \cdot\left(1-n \cdot \frac{N_{t}}{N_{t+1}}\right)$.

Gleichung 7-9

Ferner kann die bekannte Stromgrößengleichung einer umlagefinanzierten RV

$b_{t} \cdot N_{t}=r_{t} \cdot N_{t-1}$

Gleichung 7-10

umgeformt werden $\mathrm{zu}$

$r_{t}=b_{t} \cdot \frac{N_{t}}{N_{t-1}}$

Gleichung 7-11

$\mathrm{Da}$ (wegen der Annahme einer intertemporal konstanten Nettowachstumsrate des Generationenumfangs) $\frac{N_{t}}{N_{t-1}}$ durch $(1+g)$ ersetzt werden kann, lässt sich Gleichung 7-11 schreiben als

$r_{t}=b_{t} \cdot\left(1+g_{t}\right)$

Gleichung 7-12

Hierbei geht dann ein intertemporal konstanter Beitrag zur Rentenversicherung einher mit einer intertemporal konstanten absoluten Rentenhöhe (und vice versa). In diesem Fall kann vollständig auf Zeitindices verzichtet werden. 
Setzt man nun die Gleichung 7-11 (ohne Zeitindices der durchschnittlichen Zahlungsgrößen) in Gleichung 7-9 (Kinderberücksichtigung in Version 2) ein, so ergibt sich:

$b_{t}^{n}=b \cdot \frac{N_{t}}{N_{t-1}} \cdot \frac{N_{t-1}}{N_{t}}+b \cdot \frac{N_{t}}{N_{t-1}} \cdot \frac{\left(N_{t}-1\right)}{N_{t+1}} \cdot\left(1-n \cdot \frac{N_{t}}{N_{t+1}}\right) . \quad$ Gleichung 7-13

Diese Gleichung lässt sich durch elementare Operationen umformen zu

$b_{t}^{n}=2 \cdot b-\frac{1}{N_{t}} \cdot b-\frac{n \cdot b}{1+g}+\frac{n \cdot b}{N_{t+1}}$,

Gleichung 7-14

und wegen

$N_{t+1}=N_{t} \cdot(1+g)$

weiter $\mathrm{zu}$

$b_{t}^{n}=2 \cdot b-\frac{1}{N_{t}} \cdot b-b \cdot n \cdot\left(\frac{N_{t}-1}{N_{t+1}}\right)$,

Gleichung 7-15

was genau der Kinderberücksichtigung in Version 1 (Gleichung 3-11) entspricht. Somit ist für den Fall intertemporal übereinstimmender Wachstumsraten des Generationenumfangs die Gleichwertigkeit der Kinderberücksichtigung in einem Verfahren mit konstanten Beitragszahlungen und in einem mit konstanten Rentenzahlungen gezeigt. 


\subsection{Anhang 3: Zur Berechnung der bedingten Erwartungswerte}

Die Wahrscheinlichkeit, dass eine lebendgeborene Frau zum Zeitpunkt ihres 65 . Geburtstags noch lebt, beträgt (ca.) 87 v.H., der entsprechende Wert für Männer beträgt (ca.) 75 v.H. ${ }^{366}$ Es wird stochastische Unabhängigkeit der Ereignisse ,Frau lebt am 65. Geburtstag' und ,Mann lebt am 65. Geburtstag' angenommen. Aus Vereinfachungsgründen wird im weiteren unterstellt, beide Elternteile seien gleich alt. Für die Gruppe ,Eltern' sind folglich die nachstehenden Möglichkeiten des ,Lebens zum Zeitpunkt des 65. Geburtstags' mit den entsprechenden Wahrscheinlichkeiten gegeben.

\section{Tabelle 5: Berechnung der Wahrscheinlichkeiten möglicher ,Überlebenskombinationen' der Elternteile}

$\mathrm{Da}$ in dem Fall, dass bei unverheirateten Kindern beide Elternteile tot sind, auch keine Erfassung eines Rentenanspruchs mehr erfolgt (unter Vernachlässigung eventueller Waisenrenten), ist für die Ausschöpfung nur der bedingte Erwartungswert $(E), Z a h l$ der lebenden Elternteile unter der Bedingung, dass mindestens ein Elternteil lebt' relevant. Dieser lässt sich wie folgt berechnen:

$$
\begin{aligned}
& E[\text { Zahl d. leb. Elternteile } \mid \text { mind.1 E. }- \text { teil lebt }]=\frac{\{0,6525 \cdot 2+(0,2175+0,0975) \cdot 1\}}{1-0,0325} . \\
& \quad=1,6744 \approx 1,67
\end{aligned}
$$

${ }^{366}$ Vgl. STAT. BUNDESAMT (1997), Fachserie 1, Reihe 1, 1995, S. 175, Tab. 10.20. 
Gesucht ist der Wert des ,Korrektur-/ Ausschöpfungsfaktors'. Eine Vollausschöpfung läge bei 2 (lebenden) Eltern pro Kind vor. Gesucht ist also $E\left[\frac{2}{Y}\right]$ (mit $Y:$ Zahl der lebenden Eltern, wenn mindestens ein Elternteil lebt).

Nun gilt $E\left[\frac{X}{Y}\right] \approx \frac{\mu_{X}}{\mu_{Y}}-\frac{1}{\mu_{Y}{ }^{2}} \cdot \operatorname{COV}(X, Y)+\frac{\mu_{X}}{\mu_{Y}{ }^{3}} \cdot \operatorname{VAR}(Y)$ (wobei $\mu_{X(Y)}$ den Erwartungswert von $X(Y)$ bezeichnet, $\operatorname{VAR}(Y)$ die Varianz von $Y, \operatorname{COV}(X, Y)$ die Kovarianz von $X$ und $Y$ ), ${ }^{367}$ und somit (wg. $\operatorname{COV}(2, Y)=0$ und $\operatorname{VAR}(Y)=E\left[Y^{2}\right]-\left(E[Y]^{2}-\operatorname{mit} X: 2\right)$ :

$$
E\left[\frac{2}{Y}\right] \approx \frac{2}{\mu_{Y}}+\frac{2}{\mu_{Y}^{3}} \cdot\left\{E\left[Y^{2}\right]-E[Y]^{2}\right\}=\frac{2}{1,67}+\frac{2}{1,67^{3}} \cdot\left(E\left[Y^{2}\right]-1,67^{2}\right) .
$$

$\mathrm{Da}$

$$
\begin{aligned}
& E\left[Y^{2}\right]=\frac{\left\{(0,87 \cdot 0,75) \cdot 2^{2}+(0,13 \cdot 0,75+0,87 \cdot 0,25) \cdot 1^{1}\right\}}{1-0,0325} \\
& =\frac{2,925}{0,9675} \approx 3,023
\end{aligned}
$$

folgt:

$$
E\left[\frac{2}{Y}\right] \approx \frac{2}{1,67}+\frac{2}{1,67^{3}} \cdot\left(3,023-1,67^{2}\right) \approx 1,298 \approx 1,30 .
$$

Dieser Wert wird in der Arbeit zur, Ausschöpfungskorrektur' genutzt.

${ }^{367} \mathrm{Vgl}$. MOOD et al. (1974), S. 181. 


\subsection{Anhang 4: Abschätzung der impliziten Kosten der Kinderberücksichtigung in Form einer beitragsfreien Anrechnung von Kindererziehungszeiten im Rahmen der GRV}

Im Haupttext wird betont, die ausgewiesenen Kosten der Kinderberücksichtigung im Rahmen der GRV bezögen sich ausschließlich auf gegenwärtig kassenwirksame Volumina. Bedeutender seien die gegenwärtig durch Kindererziehung entstehenden (potenziellen) Rentenansprüche der Eltern. Die folgende Abschätzung dient einer Bestimmung der sich aus den Rentenzusagen ergebenden späteren Rentenforderungen. Für die Abschätzung werden zuerst einige Annahmen getroffen (und dabei erläutert resp. begründet), anschließend erfolgt eine überschlägige Berechnung.

Annahmen

1. Alle Kinder im betrachteten Zeitraum haben Mütter, die nach dem 31. Dezember 1920 geboren sind.

2. Betrachtet wird ein Rentenbezug ab dem 01. Juli 2000, so dass der ,Entgeltpunktwert' eines angerechneten Erziehungsjahres 1,0 beträgt.

Die beiden erstgenannten Annahme erscheinen bei Betrachtung der Geburten ab 1990 nicht sonderlich restriktiv.

3. Es wird mit dem westdeutschen aktuellen Rentenwert zum 01. Juli 1999 gerechnet. Dieser beträgt DM 48,29 / Monat.

Zu dem Zeitpunkt, in dem die absolute Mehrheit der seit 1990 Geborenen für ihre Eltern ,rentenwirksam' werden, ist von einer weitgehenden Angleichung der Rentenwerte von Ost- und Westdeutschland eher in Richtung des westdeutschen Wertes auszugehen, auch wenn dessen Steigerung dabei u.U. geringer ausfällt, als sie es ohne die Wiedervereinigung getan hätte.

4. Da Kinder nur rentenwirksam werden (können), wenn (mindestens) ein Elternteil das Rentenalter erreicht, wobei die Wahrscheinlichkeit für Frauen höher ist, da im Regelfall die Zurechnung bei der Frau anfällt, wird unterstellt, dass 87 v.H. der Kinder ,rentenwirksam' werden. 
Der Wert 87 v.H. entspricht der Wahrscheinlichkeit, dass ein lebendgeborenes Mädchen zum Zeitpunkt seines 65. Geburtstags noch lebt (vgl. Anhang 3 (Abschnitt 7.3). Der Wert dürfte realiter eine recht deutliche Unterschätzung des richtigen Wertes darstellen, denn die durchschnittliche Lebenserwartung von Frauen, die das gebährfähige Alter erreichen (und noch dazu in gebährfähiger körperlicher Konstitution ...), übersteigt die des Kollektivs der lebendgeborenen Frauen eindeutig. Ferner erhöht die steigende allgemeine Lebenserwartung die Wahrscheinlichkeit - die ausgewiesenen Werte sind ,Vergangenheitswerte' und unterzeichnen somit tendenziell die Wahrscheinlichkeit.

5. Der Anteil der Kinder, der ,rentenwirksam' werden kann, entspricht dem Anteil der Angestellten und Arbeiter(innen) an den Erwerbstätigen in Deutschland im April 1994. Dieser Anteil beträgt ca. 83 v.H. ${ }^{368}$

Der Rentenbezug ist an bestimmte Anforderungen geknüpft, so dass nicht alle Kinder jemals rentenwirksam werden (können). Dies gilt insbesondere für Kinder von Selbstständigen und Beamtinnen. Wegen der recht geringen Anforderungen für den Bezug einer kinderbezogenen Rente erscheint der oben ausgewiesene Von-Hundert-Wert allerdings als deutlich zu niedrig.

6. Die unter 4. und 5. ausgewiesenen Ereignisse sind voneinander unabhängig.

Für vor dem 01.01.1992 geborene Kinder erhält in der Modellrechnung ein Elternteil 1,0 Entgeltpunkt, für seitdem geborene Kinder 3,0 - was einer monatlichen Rentenzahlung von DM 48,29 bzw. DM 144,87 entspricht.

Die folgende Tabelle gibt für die Jahre seit 1990 die Zahl der lebendgeborenen Kinder an, und zusätzlich einen mit der ,Erlebenswahrscheinlichkeit' der Mutter und der Wahrscheinlichkeit der Erfüllung der allgemeinen Anspruchsvoraussetzungen für einen Rentenbezug gewichtete Kinderzahl an, sowie die sich aus diesem Wert ergebende jährliche (!) Rentensumme. (In diesem Wert ist die von der Rentenversicherung zu tragende Summe der Beitragszahlungen zur Krankenversicherung der Versicherten enthalten - als Zuschlag wird hier ein Wert von 6,8 v.H. genommen.)

${ }^{368}$ Vgl. STATISTISCHES BUNDESAMT, Statistisches Jahrbuch für Deutschland 1996 (1996), S. 110, Tab. 6.6.2; eigene Berechnung. 
Die Tabelle verdeutlicht, dass die Finanzbedeutung von Kindern für die GRV in Zukunft erheblich steigen wird. Hierzu ist zu bedenken, dass die in der obigen Tabelle ausgewiesenen jährlichen Rentenwerte an Eltern gezahlt werden, die ungefähr zur selben Zeit Rente beziehen. Die kumulierte kinderbezogene Rentensumme für jedes Jahr übersteigt mithin die Summe der oben ausgewiesenen jährlichen Rentenwerte, die sich auf die Kinder eines Jahrgangs beziehen, erheblich. Zur Verdeutlichung der finanziellen Bedeutung der rentenwirksamen Kinderbe-

\begin{tabular}{|c|c|c|c|c|}
\hline Jahr & $\begin{array}{l}\text { Lebend- } \\
\text { geborene }\end{array}$ & $\begin{array}{c}\text {,korrigierte' } \\
\text { Lebend- } \\
\text { geborene }\end{array}$ & $\begin{array}{l}\text { Entgelt- } \\
\text { punkte } \\
\text { pro Kind }\end{array}$ & $\begin{array}{l}\text { Jährlicher } \\
\text { Rentenwert }^{1)}\end{array}$ \\
\hline 1990 & 905.675 & 653.988 & 1 & 404,7 \\
\hline 1991 & 830.019 & 599.357 & 1 & 370,9 \\
\hline 1992 & 809.114 & 584.261 & 3 & $1.084,8$ \\
\hline 1993 & 798.447 & 576.559 & 3 & $1.070,4$ \\
\hline 1994 & 769.603 & 555.730 & 3 & $1.031,8$ \\
\hline 1995 & 765.221 & 552.566 & 3 & $1.025,9$ \\
\hline 1996 & 796.013 & \begin{tabular}{|l|}
574.801 \\
\end{tabular} & 3 & $1.067,2$ \\
\hline \multicolumn{5}{|c|}{ Anmerkung: 1) in Mio. DM } \\
\hline
\end{tabular}

\section{Tabelle 6: Abschätzungen zum ,Wert' der kinderbezogenen Rentenzusagen nach dem gegenwärtigen deutschen Rentenrecht}

rücksichtigung ist zu beachten, dass in den Jahren 1992 bis 1996 - bei grober Abschätzung - die folgenden beitragsbezogenen jährlichen Rentenansprüche erworben wurden: ${ }^{369,370}$

${ }^{369}$ Für die Berechnung wird unterstellt, dass der durchschnittliche rentenversicherungspflichtige Arbeitnehmer ein rentenversicherungspflichtiges Durchschnittseinkommen erhălt.

${ }^{370}$ Die Abschătzungen sind mit eher großßeren Unsicherheiten verbunden als die der kinderbezogenen Rentenanspruche. Dies liegt schon daran, dass von den beitragsbezogenen Rentenansprüchen ein großer Teil von Männern erworben wird, und somit (wegen der geringeren Restlebenserwartung nach Renteneintritt) zu größeren Teilen an Hinterbliebene ,ubergeht'. Die Abschätzungswerte stellen mithin wohl eher konservative (d.h. eher zu niedrige) Angaben dar. Dies ist z.T. auch Folge davon, dass Arbeitslose hier nicht mit einberechnet worden sind, obwohl auch sie in der Phase ihrer Arbeitslosigkeit beitragsbezogene Rentenansprulche erwerben. 


\begin{tabular}{|c|c|c|c|}
\hline \multirow{2}{*}{ Jahr } & \multicolumn{2}{|c|}{$\begin{array}{c}\text { Sozialvers.-pfl. } \\
\text { Beschäftigte } \\
\text { (in Mio.) }\end{array}$} & $\begin{array}{c}\text { jährl. korrig. } \\
\text { Rentenan- } \\
\text { sprüche } \\
\text { (in Mrd.) }\end{array}$ \\
\cline { 2 - 3 } & unkorrigiert & korrigiert & \\
\hline 1992 & 29,325 & 25,513 & 15,790 \\
\hline 1993 & 28,595 & 24,878 & 15,397 \\
\hline 1994 & 28,238 & 24,567 & 15,204 \\
\hline 1995 & 28,118 & 24,463 & 15,140 \\
\hline 1996 & 27,739 & 24,133 & 14,936 \\
\hline \multicolumn{3}{|c|}{ Anmerkungen: Werte gerundet. } \\
Quelle: BUNDESMINISTER FÜR ARBEIT UND So- \\
ZIALORDNUNG (1997b), S. 42; eigene Berechnungen. \\
\hline
\end{tabular}

Tabelle 7: Durch Beitragszahlungen in den Jahren 1992 bis 1996 erworbene jährliche Rentenansprüche

Bedeutung kinderbezogener Rentenansprüche relativiert sich damit recht deutlich. Auf Basis der Werte für 1996 lässt sich ein Grad der Beitragsorientierung des Rentenversicherungssystems von ca. 93,3 v.H. errechnen. ${ }^{371}$

$37114,936 /(14,936+1,067)=($ ca. $) 0,933$. 


\subsection{Anhang 5: Relevante Entscheidungen des Bundesverfassungsgerichts}

\section{Die Entscheidung des Bundesverfassungsgerichts vom 07.07.1992}

\section{(1 BvL 51 / 86, 50 / 87 und 1 BvR 873 / 90, 761 / 91)}

Das Bundesverfassungsgericht hat in seinem Urteil vom 07.07.1992 Stellung zur Bedeutung des Kinderhabens für die GRV bezogen. Die Beschwerdeführerinnen hatten die - ihrer Ansicht nach zu geringe - ,Kinderberücksichtigung' im Rentenversicherungsrecht ,angegriffen, weil sie nicht berücksichtige, daß die Kindererziehung wegen ihrer Bedeutung für den sogenannten Generationenvertrag in der Gesetzlichen Rentenversicherung eine den monetären Beiträgen gleichwertige Beitragsform darstelle" ${ }^{\text {372 }} \cdot{ }^{373}$ Sie verwiesen auf die große Zahl der von ihnen erzogenen Kinder und deren Beitragsleistungen für die GRV. Diese überstiegen die ihren Müttern für die Kindererziehung gezahlten Rentenleistungen um ein Vielfaches. Ferner minderten diese Zwangsabgaben der Kinder deren Möglichkeit, ihre Eltern finanziell zu unterstützen. ${ }^{374}$

Der die Bundesregierung vertretende Bundesminister für Arbeit und Sozialordnung (NORBERT BLÜM, CDU) lehnte die geforderte Gleichbehandlung, generativer' und ,monetärer' Beiträge zur RV als nicht geboten ab. „Die Beschwerdeführerinnen gingen von einem überkommenen Verständnis des Drei-GenerationenVertrages in der Gesetzlichen Rentenversicherung aus. Dieses vernachlässige nicht nur die bestehenden Außenverflechtungen, insbesondere den Gesichtspunkt der fortschreitenden europäischen Integration, sondern verkenne darüber hinaus, daß eine stark rückläufige Bevölkerungszahl jedes Alterssicherungssystem beeinträchtige. Gegen eine Gleichsetzung von monetären und ,generativen' Beiträgen spreche auch die Finanzierung im Umlageverfahren. Nur mit den von den Beitragszahlern erbrachten monetären Beiträgen seien die Leistungen der Gesetzlichen Rentenversicherung finanzierbar" ${ }^{375}$. Insbesondere wurde die Gefahr von

${ }^{372}$ BVerfGE, Vol. 87 (1992), Nr. 1 vom 07.07.1992, S. 13; vgl. auch ebd., S. 16.

${ }^{373}$ Die Ähnlichkeit der Formulierung mit den im Hauptteil (vgl. 5.1) vorgestellten Äußerungen BORCHERTs ist nicht zufällig. BORCHERT war einer der Vertreter der Beschwerdeführerinnen vor dem Bundesverfassungsgericht.

${ }^{374}$ Vgl. BVerfGE, Vol. 87 (1992), Nr. 1 vom 07.07.1992, S. 13 - 17.

${ }^{375}$ BVerfGE, Vol. 87 (1992), Nr. 1 v. 07.07.1992, S. 22. 
Wanderungsbewegungen in der europäischen Gemeinschaft betont, und auf den Zusammenhang zwischen ,Versicherungsprinzip' und ,Beitragsleistungen' verwiesen ${ }^{376}$ Ein Familienlastenausgleich sei gesamtgesellschaftlich vorzunehmen und nicht (in größerem Umfang) innerhalb der Solidargemeinschaft der Rentenversicherten.

Die Arbeitsgemeinschaft der Deutschen Familienorganisationen bezifferte die ,Transferausbeutung der Familie' im Verbund der sozialen Alterssicherungssysteme und des familiären Unterhaltsrechts auf DM $100 \mathrm{Mrd} .{ }^{377}$ und betonte, ,[d]ie Benachteiligung von Eltern in der Alterssicherung könne nur innerhalb der sozialen Alterssicherung selbst beseitigt werden"378.

Das Bundesverfassungsgericht konstatierte, es gebe keinen Zwang für den Gesetzgeber, in der RV Zeiten der Kindererziehung wie Beitragszeiten zu behandeln. „Der Gesetzgeber ist jedoch ... verpflichtet, den Mangel des Rentenversicherungssystems, der in den durch Kindererziehung bedingten Nachteilen bei der Altersversorgung liegt, in weiterem Umfang als bisher auszugleichen"379. In der Analyse der bestehenden Ausgestaltung der GRV gelangten die Verfassungsrichter zur Feststellung: „Das bestehende Alterssicherungssystem führt zu einer Benachteiligung von Personen, die sich innerhalb der Familie der Kindererziehung widmen, gegenüber kinderlosen Personen, die durchgängig einer Erwerbstätigkeit nachgehen können. Zwar macht das Rentenrecht keine Unterschiede zwischen Personen mit und ohne Familie. Rentenleistungen werden vielmehr unabhängig vom familiären Status allein an die vorherige Beitragszahlung aus dem Arbeitslohn geknüpft. Diese bestimmt den Rentenanspruch. Auf die Gründe, die zum Ausscheiden aus dem Erwerbsleben und infolgedessen zum Ausfall von Beitragszahlungen führen, kommt es nicht an. Rentenrechtlich werden Personen, die wegen Kin-

${ }^{376}$ Zwei Anmerkungen: 1. Damals hieß die ,Europäische Union' noch ,Europäische Gemeinschaft'. 2. Der Vertreter der Bundesregierung bekräftigt durch diese Feststellung, dass Wanderungsbewegungen - zumal Kinderreicher - in das Geltungsgebiet des deutschen Rentenversicherungsrechts unerwünscht sind; dies ist eine wichtige Abgrenzung zu denjenigen, die in der Migration eine Stütze der GRV sehen. Der Vertreter des VDR verwies in seiner Stellungnahme im Rahmen desselben Verfahrens jedenfalls darauf, dass der ,Außenwanderungssaldo' bedeutsam für die demographische Entwicklung sei, die wiederum ,unbestreitbar ein wesentlicher Faktor für die finanzielle Entwicklung der gesetzlichen Rentenversicherung, aber auch für die Finanzierbarkeit des Sozialleistungssystems überhaupt" (BVerfGE, Vol. 87 (1992), Nr. 1 v. 07.07.1992, S. 26f.) sei.

377 Vgl. BVerfGE, Vol. 87 (1992), Nr. 1 v. 07.07.1992, S. $28 f$.

${ }^{378}$ BVerfGE, Vol. 87 (1992), Nr. 1 v. 07.07.1992, S. 30.

379 BVerfGE, Vol. 87 (1992), Nr. 1 v. 07.07.1992, S. 1. 
dererziehung aus dem Erwerbsleben ausscheiden, wie jeder andere nicht Erwerbstätige behandelt.

Im Unterschied zu den Gründen, die sonst für die Erwerbslosigkeit und damit den Ausfall von Beitragszahlungen ursächlich sein mögen, hat die Kindererziehung allerdings bestandssichernde Bedeutung für das System der Altersversorgung. ... Dabei kann angesichts der Breitenwirkung der Rentenversicherung vernachlässigt werden, daß nicht jedes Kind später zum Beitragszahler wird"380.

Die Verfassungsrichter konstatierten allerdings, dass die Nachteile nicht allesamt Folge des Rentenrechts seien. Dementsprechend müssten sie nicht nur dort behoben werden. ${ }^{381}$ Ferner seien im Umlageverfahren, "das verfassungsrechtlich nicht zu beanstanden ist, .. Kindererziehung und Beitragszahlung nicht gleichartig" 382 . Diese Ungleichartigkeit rechtfertige auch eine unterschiedliche Behandlung in der RV. Bei jeder weiteren Reform sei eine Verringerung der Benachteiligung der Familie im Rahmen der RV sicherzustellen. Dabei stehe der Schutz der Rentenanwartschaften „einer maßvollen Umverteilung ... zu Lasten kinderloser und kinderarmer Personen nicht entgegen"383.

Anmerkung: Im besprochenen Verfahren hatte das Verfassungsgericht auch die Frage zu klären, inwieweit die von der Bundesregierung gewăhlte Regelung einer sukzessiven Einfuhrung einer Anrechnung der Kindererziehungszeit (von einem Jahr) rechtmäßig war. (Das Gericht bejahte die Verfassungsmäßigkeit.) Da dieser Punkt fur die vorliegende Arbeit von geringer Bedeutung ist, wird auf die detaillierte Darstellung der Begründung sowohl der Verfassungsbeschwerde als auch des diesbezuglichen Urteils (sowie der Argumentation im Rahmen der Verhandlung vor dem Verfassungsgericht) verzichtet.

\footnotetext{
${ }^{380}$ BVerfGE, Vol. 87 (1992), Nr. 1 v. 07.07.1992, S. 37.

${ }^{381}$ Vgl. BVerfGE, Vol. 87 (1992), Nr. 1 v. 07.07.1992, S. 39.

${ }^{382}$ BVerfGE, Vol. 87 (1992), Nr. 1 v. 07.07.1992, S. 40.

${ }^{383}$ BVerfGE, Vol. 87 (1992), Nr. 1 v. 07.07.1992, S. 41.
} 


\section{Die Entscheidung des Bundesverfassungsgerichts vom 12.03.1996 ${ }^{384}$}

(1 BvR 609, 692 / 90)

Das Bundesverfassungsgericht hat in seinem Urteil vom 12.03.1996 die Anrechnung von durch Kindererziehungszeiten induzierten Entgeltpunkten auf durch eventuelle Beitragsleistungen in dieser Zeit erworbene Entgeltpunkte für mit Artikel 3 Absatz 1 des Grundgesetzes unvereinbar erklärt, und dem Gesetzgeber eine Frist bis zum 30.06.1998 zur Korrektur der Regelung gegeben.

Die Begründung für die ,Anrechnungslösung' aus dem Gesetzgebungsverfahren war, dass Eltern durch die Kindererziehung nicht ihre soziale Absicherung verlieren sollen. Die Beschwerdeführerinnen gegen die Regelungen verwiesen darauf, dass die erbrachte Erziehungsleistung nicht infolge von (paralleler) Erwerbstätigkeit zu einer ,Nichtleistung' werde, während der die Gesetzesregelung verteidigende Bundesminister für Arbeit und Sozialordnung auf das - für die Rentenversicherung typische - ,Lückenschließerprinzip' verwies, dem die Regelung diene.

In seiner Urteilsbegründung hob das Bundesverfassungsgericht hervor, dass auch Leute, die zu keinem Zeitpunkt Pflichtbeiträge zur GRV geleistet haben, einen Anspruch auf die Anrechnung von Kindererziehungszeiten besitzen; dies zeige, dass die Anrechnung von Kindererziehungszeiten nicht ausschließlich eine ,Lükkenschließerfunktion' erfülle.

Die Erziehung von Kindern diene dem Erhalt der Rentenversicherung, auch wenn nicht alle Kinder später zur Rentenversicherung Beiträge zahlten. Aufgrund ihrer Unterschiedlichkeit sei es nicht verfassungsmäßig geboten, Kindererziehung und Beitragsleistungen gleich zu behandeln, eine rentenversicherungspflichtige Erwerbstätigkeit einer Erziehungsperson schmälere den Wert der Kindererziehung jedoch nicht (und auch nicht zeitnahe freiwillige Einzahlungen zur Rentenversicherung).

${ }^{384}$ Zum Folgenden vgl. BVerfGE, Vol. 94 (1996), Nr. 6 v. 12.03.1996, S. 241 - 267. 


\section{Literaturverzeichnis}

Agee, Mark D., Crocker, Thomas D. (1996), „Parents' Discount Rates for Child Quality", Southern Economic Journal, Vol. 63 (Juli 1996), S. 36 - 50.

Ahn, Namkee (1995), „Measuring the Value of Children by Sex and Age Using a Dynamic Programming Model", Review of Economic Studies, Vol. 62, S. 361 379.

Albers, Willi (1997), „Die Alterssicherung kindererziehender Mütter und Witwen", Sozialer Fortschritt, Vol. 46, S. 137 - 140.

Altonji, Joseph G., Hayashi, Fumio, Kotlikoff, Laurence J. (1997), „Parental Altruism and Inter Vivos Transfers: Theory and Evidence", Journal of Political Economy, Vol. 105, S. $1121-1166$.

Appelbaum, Elie, Katz, Eliakim (1991), „The Demand for Children in the Absence of Capital and Risk Markets: A Portfolio Approach", Oxford Economic Papers, Vol. 43, S. 292 - 304.

Arthur, W. Brian, McNicoll, Geoffrey (1977), „Optimal Time Paths with Age-Dependence: A Theory of Population Policy“, Review of Economic Studies, Vol. 44, S. $111-123$.

Ball, Helmut (1984), Zukunftsvorsorge und ökonomische Entwicklung - Entscheidungen über Kapitalbildung, Versicherung, Ausbildung, Arbeit und Generationenvertrag aus einzel- und gesamtwirtschaftlicher Sicht, [Hrsg. von d. Stiftung Gesellschaft u. Unternehmen] Frankfurt / Main, New York.

Becker, Gary S. (1960), „An Economic Analysis of Fertility”, in: National Bureau of Economic Research (Hrsg.), Demographic Change in Developed Countries, Princeton, 1960; wiederabgedruckt in: Gary S. Becker, The Economic Approach to Human Behavior, Chicago, 1976, Paperback-Ausgabe 1978; hier: Chap. 9, S. $171-194$.

Becker, Gary S. (1973/1974), „A Theory of Marriage“, Journal of Political Economy, Vol. 81 (1973), S. 813 - 846 zusammen mit Journal of Political Economy, Vol. 82 (1974), S. S11 - S26; (leicht abgeändert) abgedruckt auch in: Theodore W. Schultz (Hrsg.), Economics of the Family - Marriage, Children, and Human 
Capital, Chicago, London, 1974, S. 299 - 344 sowie in Becker, Gary S., The Economic Approach to Human Behavior, Chicago, 1976, Chap. 11.

Becker, Gary S. (1974), „A Theory of Social Interactions”, Journal of Political Economy, Vol. 82, S. 1063 - 1093.

Becker, Gary S. (1976), „Altruism, Egoism, and Genetic Fitness: Economics and Sociobiology", Journal of Economic Literature, Vol. 14, S. 817 - 826.

Becker, Gary S. (1977), „Reply to Hirshleifer and Tullock”, Journal of Economic Literature, Vol. 15, S. 506f.

Becker, Gary S. (1981), A Treatise on the Family, Cambridge / MA, London; hier: 1. Paperback-Ausgabe, 1993, 3. Auflage, 1994.

Becker, Gary S. (1989), „On the Economics of the Family: Reply to a Skeptic”, American Economic Review, Vol. 79, S. 514 - 518.

Becker, Gary S., Barro, Robert J. (1988), „A Reformulation of the Economic Theory of Fertility", Quarterly Journal of Economics, Vol. 103, S. 1 - 25.

Becker, Gary S., Lewis, H. Gregg (1974), „Interaction between Quantity and Quality of Children", in: Theodore W. Schultz (Hrsg.), Economics of the Family Marriage, Children, and Human Capital, Chicago, London, 1974, S. 81 - 90; abgedruckt auch in: Journal of Political Economy, Vol. 81 (1973), S. S279 - S288.

Becker, Gary S., Murphy, Kevin M. (1988), „The family and the State”, Journal of Law and Economics, Vol. 31, S. 1 - 18.

Becker, Gary S., Tomes, Nigel (1979), „An Equilibrium Theory of the Distribution of Income and Intergenerational Mobility", Journal of Political Economy, Vol. 87, S. $1153-1189$.

Becker, Gary S., Murphy, Kevin M., Tamura, Robert (1990), „Human Capital, Fertility, and Economic Growth", Journal of Political Economy, Vol. 98, S. S12 S37. 
Behrman, Jere R., Rosenzweig, Mark R., Taubman, Paul (1994), „Endowments and the Allocation of Schooling in the Family and the Marriage Market: The Twins Experiment", Journal of Political Economy, Vol. 102, S. 1131 - 1175.

Ben-Porath, Yoram (1974), „Economic Analysis of Fertility in Israel“, in: Theodore W. Schultz (Hrsg.), Economics of the Family - Marriage, Children, and Human Capital, Chicago, London, 1974, S. 189 - 220; abgedruckt auch in: Journal of Political Economy, Vol. 81 (1973), S. S202 - S233.

Benham, Lee (1974), „Benefits of Women's Education within Marriage“, in: Theodore W. Schultz (Hrsg.), Economics of the Family - Marriage, Children, and Human Capital, Chicago, London, 1974, S. 375 - 389; abgedruckt auch in: Journal of Political Economy, Vol. 82 (1974), S. S57 - S71.

Bernheim, B. Douglas (1991), „How Strong Are Bequest Motives? Evidence Based on Estimates of the Demand for Life Insurance and Annuities", Journal of Political Economy, Vol. 99, S. 899 - 927.

Bernheim, B. Douglas, Stark, Oded (1988), „Altruism Within the Family Reconsidered: Do Nice Guys Finish Last?", American Economic Review, Vol. 78, S. $1034-1045$.

Bernheim, B. Douglas, Shleifer, Andrei, Summers, Lawrence H. (1985), „The Strategic Bequest Motive", Journal of Political Economy, Vol. 93, S. 1045 - 1076.

Berntsen, Roland (1992), Dynamik in der Einkommensverteilung privater Haushalte - Eine empirische Längsschnittanalyse für die Bundesrepublik, Frankfurt / Main, New York.

Berthold, Norbert (1992), „Familie, Fertilität und Einkommensverteilung”, Jahrbücher für Nationalökonomie und Statistik, Vol. 209, S. 523 - 541.

Berthold, Norbert, Schmid, Cornelia (1997), „Der Generationenvertrag - ein Auslaufmodell?", List Forum für Wirtschafts- und Finanzpolitik, Vol. 23, S. 143 167.

Berthold, Norbert, Thode, Eric (1996), „Auslagerung versicherungsfremder Leistungen - Ausweg oder Kreisverkehr?", Wirtschaftsdienst, Vol. 76, S. 350 - 358. 
Blau, David M., Robins, Philip K. (1988), „Child-Care Costs and family Labor Supply", Review of Economics and Statistics, Vol. 70, S. 374 - 381.

Blossfeld, Hans-Peter, Huinink, Johannes (1989), „Die Verbesserung der Bildungs- und Berufschancen von Frauen und ihr Einfluß auf den Prozeß der Familienbildung", Zeitschrift für Bevölkerungswissenschaft, Vol. 15, S. 383 - 404.

Blossfeld, Hans-Peter, Huinink, Johannes, Rohwer, Götz (1991), „Wirkt sich das steigende Bildungsniveau der Frauen tatsächlich negativ auf den Prozeß der Familienbildung aus?", Zeitschrift für Bevölkerungswissenschaft, Vol. 17, S. 337 351.

Borchert, Ernst-Jürgen (1981), Die Berücksichtigung familiärer Kindererziehung im Recht der gesetzlichen Rentenversicherung, Sozialpolitische Schriften, Heft 42, Berlin.

Borchert, Jürgen (1993), Renten vor dem Absturz - Ist der Sozialstaat am Ende?, Frankfurt / Main, 1993, hier: Auflage 1994.

Borchert, Jürgen (1994), „Prolegomena zur familienpolitischen Strukturreform des Sozialstaats", Zeitschrift für Sozialreform, Vol. 40, S. 440 - 462.

Börsch-Supan, Axel (1992), „Population Aging, Social Security Design, and Early Retirement", Journal of Institutional and Theoretical Economuics, Vol. 148, S. $533-557$.

Bowles, Samuel (1972), „Schooling and Inequality from Generation to Generation", Journal of Political Economy, Vol. 80, S. S219 - S251.

Breyer, Friedrich (1990), „Das Äquivalenzprinzip in der Rentenversicherung aus wohlfahrtsökonomischer Sicht", Finanzarchiv, Neue Folge, Vol. 48, S. 127 - 142.

Breyer, Friedrich (1992), „Die Zukunft umlagefinanzierter Alterssicherungssysteme bei schrumpfender Bevölkerung", Wirtschaftswissenschaftliches Studium (WiSt), Vol. 21, S. $106-112$.

Breyer, Friedrich, Schulenburg, J.-M. Graf von der (1987a), „Voting on Social Security: The Family as Decision-Making Unit”, Kyklos, Vol. 40, S. 529 - 547. 
Breyer, Friedrich, Schulenburg, J.-M. Graf von der (1987b), „Family Structure and Intergenerational Transfers in Social Health Insurance: A Public Choice Model", in: Rüdiger Pethig Ulrich Schlieper (Hrsg.), Efficiency, Institutions, and Economic Policy, Berlin u.a.O., S. 63 - 80.

Breyer, Friedrich, Schulenburg, J.-M. Graf von der (1989), „Umlagefinanzierte Rentenversicherung bei abnehmender Bevölkerung unter den Bedingungen eines demokratischen Wahlmechanismus", Jahrbuch für Neue Politische Ökonomie, Vol. 8, S. $126-139$.

Browning, Martin (1992), „Children and Household Economic Behavior”, Journal of Economic Literature, Vol. 30, S. 1434 - 1475.

Bruce, Neil, Waldman, Michael (1990), „The Rotten-Kid Theorem Meets the Samaritarian's Dilemma", Quarterly Journal of Economics, Vol. 105, S. 155 - 165.

Brüderl, Josef, Klein, Thomas (1991), „Bildung und Familiengründung: Institutionen- versus Niveaueffekte", Zeitschrift für Bevölkerungswissenschaft, Vol. 17, S. $323-335$.

Bundesminister für Arbeit und Sozialordnung (Hrsg.) (1995), Arbeits- und Sozialstatistik - Hauptergebnisse 1995, Bonn.

Bundesministerium für Arbeit und Sozialordnung (Hrsg.) (1997a), Materialband zum Sozialbudget 1997, Bonn.

Bundesminister für Arbeit und Sozialordnung (Hrsg.) (1997b), Arbeits- und Sozialstatistik - Hauptergebnisse 1997, Bonn.

Bundesministerium für Familie und Senioren (Hrsg.) (1994), Familien und Familienpolitik im geeinten Deutschland - Fünfter Familienbericht, Bonn, Bonner Universitäts-Druckerei, Deutscher Bundestag, Drucksache 12 / 7560.

Bundesministerium für Familie, Senioren, Frauen und Jugend (Hrsg.) (1995), Lebenshaltungsaufwendungen für Kinder, Schriftenreihe, Band 43, Stuttgart u.a.O.

Butz, William P., Ward, Michael P. (1979), „The Emergence of Countercyclical U.S. Fertility", American Economic Review, Vol. 69, S. 318 - 328. 
Cigno, Alessandro (1986), „Fertility and the Tax-Benefit System: A Reconsideration of the Theory of Family Taxation", Economic Journal, Vol. 96, S. 1035 1051.

Cigno, Alessandro (1991), Economics of the Family, Oxford u.a.O.

Cleveland, Gordon, Gunderson, Morley, Hyatt, Douglas (1996), „Child care costs and the employment decision of women: Canadian evidence", Canadian Journal of Economics, Vol. 29, S. 132 - 151.

Connelly, Rachel (1992), „The Effect of Child Care Costs on Married Women's Labor Force Participation", Review of Economics and Statistics, Vol. 74, S. 83 90 .

Cornelius, Ivar (1988), „Sozialökonomische Problemlagen von Familien in unterschiedlichen Lebensphasen", Sozialer Fortschritt, Vol. 37, S. 247 - 250.

Cox, Donald, Jakubson, George (1995), „The connection between public transfers and private interfamily transfers", Journal of Public Economics, Vol. 57, S. 129 167.

Deaton, Angus S., Muellbauer, John (1986), „On Measuring Child Costs: With Applications to Poor Countries", Journal of Political Economy, Vol. 94, S. 720 744.

Demeny, Paul (1986), „Bevölkerung und die unsichtbare Hand”, Zeitschrift für Bevölkerungswissenschaft, Vol. 12, S. 437 - 456.

Dinkel, Reiner (1981), „Kinder- und Alterslastenausgleich bei abnehmender Bevölkerung", Finanzarchiv, Neue Folge, Vol. 39, S. 134 - 147.

Dinkel, Reiner (1984a), „Demographical Influences on Social Security Burden”, in: Gunter Steinmann (Hrsg.), Economic Consequences of Population Change in Industrialized Countries (Studies in contemporary economics, Vol. 8), Berlin u.a.O., S. 87 - 107.

Dinkel, Reiner (1984b), Die Auswirkungen eines Geburten- und Bevölkerungsrückgangs auf Entwicklung und Ausgestaltung von gesetzlicher Alterssicherung und Familienlastenausgleich, Sozialpolitische Schriften, Heft 49, Berlin. 
Dorbritz, Jürgen (1998), „Trends der Geburtenhäufigkeit in Niedrig-FertilitätsLändern und Szenarien der Familienbildung in Deutschland", Zeitschrift für Bevölkerungswissenschaft, Vol. 23, S. $179-210$.

Dorbritz, Jürgen, Fleischhacker, Jochen (1995), „Der Übergang von der Bevölkerungs- zur Familienpolitik in den neuen Bundesländern - ein Beitrag zum familienpolitischen Diskurs in Deutschland", Zeitschrift für Bevölkerungswissenschaft, Vol. 20, S. 159 - 185.

Dorbritz, Jürgen, Schwarz, Karl (1996), „Kinderlosigkeit in Deutschland - ein Massenphänomen? Analysen zu Erscheinungsformen und Ursachen", Zeitschrift für Bevölkerungswissenschaft, Vol. 21, S. 231 - 261.

Eckstein, Zvi, Eichenbaum, Martin, Peled, Dan (1985), „Uncertain lifetimes and the welfare enhancing properties of annuity marktes and social security", Journal of Public Economics, Vol. 26, S. 303 - 326.

Eekhoff, Johann (1997), „Die Zukunft der sozialen Sicherung in einem integrierten Europa - Konsequenzen für die Rentenreform?", in: Wie sicher ist unsere Zukunft? Entwicklungsperspektiven der sozialen Sicherung, Tagungsband zur Jahreshauptversammlung der Arbeitsgemeinschaft Deutscher Wirtschaftswissenschaftlicher Forschungsinstitute e.V. im April 1997 in Bonn, Duncker \& Humblot, Berlin (zugl.: Beihefte zur Konjunkturpolitik - Zeitschrift für angewandte Wirtschaftsforschung, Heft 46), S. 27 - 48.

Eggen, Bernd (1997), „Einkommenslagen und wirtschaftlich schwierige Situationen von Ehepaaren ohne Kinder und mit Kindern in Ost- und Westdeutschland", Sozialer Fortschritt, Vol. 46, S. 68 - 73.

Ehrlich, Issac, Zhong, Jian-Guo (1998), „Social Security and the Real Economy: An Inquiey into Some Neglected Issues", American Economic Review, Papers and Proceedings, Vol. 88, S. 151 - 157.

Eitenmüller, Stefan (1998), „Die Rendite im Umlageverfahren und die Kosten eines Wechsels zum Kapitaldeckungsverfahren", Vorlage zu Vortrag auf dem VDR Pressekontaktseminar 1998 am 24. und 25.08.1998 in Bad Lauterberg.

Engstler, Heribert, Lüscher, Kurt (1991), „Späte erste Mutterschaft. Ein neues biographisches Muster der Familiengründung?", Zeitschrift für Bevölkerungswissenschaft, Vol. 17, S. 433 - 460. 
Euler, Manfred (1993), „Aufwendungen für Kinder“, Wirtschaft und Statistik, Nr. $10 /$ 1993, S. 759 - 769.

Famulla, Rainer, Spremann, Klaus (1980), „Generationenverträge und Rentenversicherung als Ponzi GmbH", Zeitschrift für öffentliche und gemeinwirtschaftliche Unternehmen, Vol. 3, S. 379 - 403.

Felderer, Bernhard (1992), „Does a Public Pension System Reduce Saving Rates and Birth Rates?", Journal of Institutional and Theoretical Economics, Vol. 148, S. $314-325$.

Feucht, Ralf (1995), „Lasten der umlagefinanzierten Altersrente vor dem Hintergrund eines demographischen Wandels", Zeitschrift für Bevölkerungswissenschaft, Vol. 20, S. 215 - 231.

Fleisher, Belton M., Rhodes (Jr.), George F. (1979), „Fertility, Women's Wage Rates, and Labor Supply", American Economic Review, Vol. 69, S. 14 - 24.

Fogel, Robert William, Engerman, Stanley L. (1974), Time on the Cross - The Economics of American Negro Slavery, New York, London, hier: PaperbackAusgabe 1995.

Freiden, Alan (1974), „The U.S. Marriage Market", in: Theodore W. Schultz (Hrsg.), Economics of the Family - Marriage, Children, and Human Capital, Chicago, London, 1974, S. 352 - 371; abgedruckt auch in: Journal of Political Economy, Vol. 82 (1974), S. S34 - S53.

Frey, Bruno S. (1997), „A Constitution for Knaves Crowds Out Civic Virtues”, Economic Journal, Vol. 107, S. 1043 - 1053.

Frey, Bruno S., Oberholzer-Gee, Felix, Eichenberger, Rainer (1996), „The Old Lady Visits Your Backyard: A Tale of Morals and Markets", Journal of Political Economy, Vol. 104, S. 1297 -1313.

Fritzsche, Bernd (1986), „Kindergeld aus Rentenkassen?”, Sozialer Fortschritt, Vol. 35, S. 202 - 205.

Gaillinger, Heidi (1994), „Interfamiliäre Umverteilungseffekte in Alterssicherungssystemen", Wirtschaftswissenschaftliches Studium (WiSt), Vol. 23, S. 635 638. 
Galler, Heinz P. (1988), „Familiale Lebenslagen und Familienlastenausgleich“, in: Bernhard Felderer (Hrsg.), Familienlastenausgleich und demographische Entwicklung, Schriften des Vereins für Socialpolitik, Neue Folge, Band 175, Berlin, S. $83-112$.

Gallon, Thomas - Peter (1993a), „Logische Inkonsistenzen des ,Elternrenten'Modells", Sozialer Fortschritt, Vol. 42, S. 10 - 16.

Gallon, Thomas - Peter (1993b), „Reform der Erziehungszeiten im Rentenrecht als gesellschaftliche Aufgabe", Sozialer Fortschritt, Vol. 42, S. 218 - 222.

Glismann, Hans H., Horn, Ernst-Jürgen (1995a), „Die staatliche Alterssicherung in Deutschland: Einige ordnungspolitische Anmerkungen", Orientierungen zur Wirtschafts- und Gesellschaftspolitik, Vol. 1 / 63, S. 28 - 33.

Glismann, Hans H., Horn, Ernst-Jürgen (1995b), „Warum die Renten nicht sicher sind”, FAZ Nr. 174 / '95 vom 29.07.1995, S. 13.

Glismann, Hans H., Horn, Ernst-Jürgen (1995c), „Die Krise des deutschen Systems der staatlichen Alterssicherung", ORDO, Bd. 46, S. 309 - 344.

Glombik, Manfred (1995), „Der Generationenvertrag auf dem Prüfstand”, Orientierungen zur Wirtschafts- und Gesellschaftspolitik, Vol 1 / 63, S. 34f.

Görzig, Bernd (1977), „Die Auswirkungen des Geburtenrückgangs auf die Nachfragestruktur", in: Bundesinstitut für Bevölkerungsforschung (Hrsg.), Materialien zur Bevölkerungswissenschaft, Heft 9, Ursachen des Bevölkerungsrückgangs und seine Konsequenzen für die deutsche Wirtschaft, Tagung 7. - 10.12.1977, ohne Ort, ohne Jahr, S. 55 - 65.

Gostomski, Christian Babka von (1998), „Machen Kinder Ehen glücklich?”, Zeitschrift für Bevölkerungswissenschaft, Vol. 23, S. 151 - 177.

Grohmann, Heinz (1986), „Probleme einer Abschätzung des für ein Kapitaldeckungsverfahrens in der gesetzlichen Rentenversicherung notwendigen Deckungskapitals - Theoretische Überlegungen und empirische Ergebnisse", in: Bernhard Felderer (Hrsg.), Kapitaldeckungsverfahren versus Umlageverfahren, Schriften des Vereins für Socialpolitik, Neue Folge, Band 163, Berlin, S. 67 - 89. 
Hackmann, Johannes (1990), „Freiheit und Sicherheit in Systemen der sozialen Sicherung. Zu einer Theorie sozialer Sicherungssysteme", in: Kurt Schmidt (Hrsg.), Finanzierungsprobleme der sozialen Sicherung I, Schriften des Vereins für Socialpolitik, Band 194 / I, Berlin, S. 41 - 144.

Hackmann, Johannes (1994), „Die einkommensteuerliche Berücksichtigung des Existenzminimums", Betriebsberater, Beilage 19 zu Heft 26, Vol. 19, S. 1* - 27*.

Hanesch, Walter et al. (Hrsg.) (1994), Armut in Deutschland - Der Armutsbericht des DGB und des Paritätischen Wohlfahrtsverbandes, Reinbek.

Hase, Friedhelm (1992), „Benachteiligung der Familie durch die Rentenversicherung?", Wirtschaftsdienst, Vol. 72, S. 526 - 530.

Hashimoto, Masanori (1974), „Economics of Postwar Fertility in Japan“, in: Theodore W. Schultz (Hrsg.), Economics of the Family - Marriage, Children, and Human Capital, Chicago, London, 1974, S. 225 - 249; abgedruckt auch in: Journal of Political Economy, Vol. 82 (1974), S. S170 - S194.

Hauser, Richard, Hübinger, Werner (1993), Arme unter uns, Teil 1: Ergebnisse und Konsequenzen der Caritas-Armutsuntersuchung, Lambertus, Freiburg i. Brsg.

Hauser, Richard (1995), „Die Entwicklung der Einkommenslage von Familien über zwei Dekaden - einige empirische Grundlagen zur Würdigung der deutschen Familienpolitik", in: Gerhard Kleinhenz (Hrsg.), Soziale Ausgestaltung der Marktwirtschaft, Festschrift zum 65. Geburtstag für Prof. Dr. Heinz Lampert, Sozialpolitische Schriften, Heft 65, 1995, S. 133 - 150.

Haveman, Robert, Wolfe, Barbara (1995), „The Determinants of Children's Attainments: A Review of Methods and Findings", Journal of Economic Literature, Vol. 33, S. 1879 -1902.

Heckmann, James J. (1974), „Effects of Child-Care Programs on Women's Work Effort", in: Theodore W. Schultz (Hrsg.), Economics of the Family - Marriage, Children, and Human Capital, Chicago, London, 1974, S. 491 - 518; abgedruckt auch in: Journal of Political Economy, Vol. 82 (1974), S. S136 - S163.

Henderson, A. M. (1949), „The Cost of a Family”, Review of Economic Studies, Vol. 17 (1949 / 50), S. 127 - 148. 
Hess, Gregory D., Orphanides, Athanasios (1996), „Taxation and Intergenerational Transfers with Family-Size Heterogeneity: Do Parents with More Children Prefer Higher Taxes?", Journal of Money, Credit, and Banking, Vol. 28, S. 162 177.

Hirshleifer, Jack (1977), „Shakespeare vs. Becker on Altruism: The Importance of Having the Last Word", Journal of Economic Literature, Vol. 15, S. 500ff.

Hockauf-Schneider, Reinhilde (1988), „Kinder als Armutsursache von Lohnempfängern?", Zeitschrift für Bevölkerungswissenschaft, Vol. 14, S. 23 - 48.

Höhn, Charlotte (1986), „Einflußfaktoren des generativen Verhaltens”, Zeitschrift für Bevölkerungswissenschaft, Vol. 12, S. 309 - 323.

Höhn, Charlotte (1989), „Zum Konzept bevölkerungsrelevanter Politiken auf dem Hintergrund eines Bevölkerungsrückgangs", Zeitschrift für Bevölkerungswissenschaft, Vol. 15, S. 211 - 220.

Höpflinger, Francois (1982), „Geschlechtsspezifische Unterschiede im Kinderwunsch", Zeitschrift für Bevölkerungswissenschaft, Vol. 8, S. 15 - 30.

Hofmann, Jürgen (1996), „,Versicherungsfremde Leistungen' und Bundeszuschuß zur gesetzlichen Rentenversicherung“, Sozialer Fortschritt, Vol. 45, S. 126 - 128.

Hohnerlein, Eva-Maria (1992), „Kompensatorische Regelungen der gesetzlichen Alterssicherungssysteme bei durchbrochenen Erwerbsbiographien - Ansätze zur Anerkennung unbezahlter Familienarbeit in Europa", Zeitschrift für Sozialreform, Vol. 38, S. 589 - 604.

Holzer, Werner, Münz, Rainer (1996), „Kinderwunsch in Österreich”, Zeitschrift für Bevölkerungswissenschaft, Vol. 21, S. 69 - 102.

Homburg, Stefan, Gräff, Carsten (1988), „Zur ökonomischen Begründbarkeit eines Familienlastenausgleichs", in: Bernhard Felderer (Hrsg.), Familienlastenausgleich und demographische Entwicklung, Schriften des Vereins für Socialpolitik, Neue Folge, Band 175, Berlin, S. 13 - 28.

Jürgens, Hans W. (1977), „Kinderwunsch und Wirklichkeit - untersucht an Längsschnittuntersuchungen in der Bundesrepublik Deutschland", in: Bundesinstitut für Bevölkerungsforschung (Hrsg.), Materialien zur Bevölkerungswissen- 
schaft, Heft 9, Ursachen des Bevölkerungsrückgangs und seine Konsequenzen für die deutsche Wirtschaft, Tagung 7. - 10.12.1977, ohne Ort, ohne Jahr, S. 163 171.

Kane, Thomas T. (1986), „The fertility asimilation of guestworker population in the Federal Republic of Germany: 1961 - 1981", Zeitschrift für Bevölkerungswissenschaft, Vol. 12, S. 99 - 131.

Kapteyn, Arie, Praag, Bernard van (1976), „A New Approach to the Construction of Family Equivalence Scales", European Economic Review, Vol. 7, S. 313 - 335.

Kaufmann, Franz-Xaver (1990), „Ursachen des Geburtenrückgangs in der Bundesrepublik Deutschland und Möglichkeiten staatlicher Gegenmaßnahmen", Zeitschrift für Bevölkerungswissenschaft, Vol. 16, S. 383 - 396.

Kemper, Franz-Josef (1982), „Sozio-ökonomische und siedlungsstrukturelle Faktoren für die Familiengröße", Zeitschrift für Bevölkerungswissenschaft, Vol. 8, S. $225-242$.

Klein, Thomas (1984), „Der Einfluß von Familie und Kindern auf die wohlfahrtsgerechten Abstufungen von Sozialeinkommen", Zeitschrift für Bevölkerungswissenschaft, Vol. 10, S. 89 - 108.

Klein, Thomas, Lengsfeld, Wolfgang (1985), „Sozialstrukturelle Ursachen des Geburtenrückgangs", Zeitschrift für Bevölkerungswissenschaft, Vol. 11, S. 57 74.

Klerman, Jacob Alex, Leibowitz, Arleen (1990), „Child Care and Women's Return To Work After Childbirth", American Economic Review, Papers and Proceedings, Vol. 80, S. 284 - 288.

Kremer, Michael (1997), „How Much Does Sorting Increase Inequality?”, Quarterly Journal of Economics, Vol. 112, S. 115 - 139.

Laitner, John, Juster, F. Thomas (1996), „New Evidence on Altruism: A Study of TIAA-CREF Retirees", American Economic Review, Vol. 86, S. 893 - 908.

Lampert, Heinz (1993), „Probleme, ungelöste und neue Aufgaben der Familienpolitik", in: Albrecht Iwersen, Egon Tuchtfeldt (Hrsg.): Sozialpolitik vor neuen Aufgaben, Bern, S. 347 - 363. 
Lapan, Harvey E., Enders, Walter (1990), „Endogenous Fertility, Ricardian Equivalence, and Debt Management Policy", Journal of Public Economics, Vol. 41, S. 227 - 248.

Lindbeck, Assar (1997), „The Swedish Experiment”, Journal of Economic Literature, Vol. 35, S. 1273 - 1319.

Lüdeke, Reinar (1995), „Kinderkosten, umlagefinanzierte Rentenversicherung, Staatsverschuldung und intergenerative Einkommensverteilung", in: Gerhard Kleinhenz (Hrsg.), Soziale Ausgestaltung der Marktwirtschaft, Festschrift zum 65. Geburtstag für Prof. Dr. Heinz Lampert, Sozialpolitische Schriften, Heft 65, Berlin, S. 150 - 183.

Macunovich, Diane J. (1996), "A Review of Recent Developments in the Economics of Fertility", in: Paul L. Menchik (Hrsg.), Household and Family Economics, Boston u.a.O., S. 91 - 150.

Michael, Robert T. (1974), „Education and the Derived Demand for Children“, in: Theodore W. Schultz (Hrsg.), Economics of the Family - Marriage, Children, and Human Capital, Chicago, London, 1974, S. 120 - 156; abgedruckt auch in: Journal of Political Economy, Vol. 81 (1973), S. S128 - S164.

Mincer, Jacob, Polachek, Solomon (1974), „Family Investments in Human Capital: Earnings of Women", Journal of Political Economy, Vol. 82, S. S76 - S110; abgedruckt auch in: Theodore W. Schultz (Hrsg.), Economics of the Family Marriage, Children, and Human Capital, Chicago, London, 1974, S. 397 - 429.

Mood, Alexander M., Graybill, Franklin A., Boes, Duane C. (1974), Introduction to the Theory of Statistics, Third Edition, 19. Auflage, Singapore u.a.O. (1988).

Molt, Walter (1977), „Geburtenrückgang als Konsequenz des Wertwandels”, in: Bundesinstitut für Bevölkerungsforschung (Hrsg.), Materialien zur Bevölkerungswissenschaft, Heft 9, Ursachen des Bevölkerungsrückgangs und seine Konsequenzen für die deutsche Wirtschaft, Tagung 7. - 10.12.1977, ohne Ort, ohne Jahr, S. $133-147$.

Mroz, Thomas A. (1987), „The Sensitivity of an Empirical Model of Married Women's Hours of Work to Economic and Statistical Assumptions", Econometrica, Vol. 55, S. 765 - 799. 
Muellbauer, John (1974), „Household Composition, Engel Curves and Welfare Comparisons between Households", European Economic Review, Vol. 5, S. 103 122.

Muellbauer, John (1977), „Testing the Barten Model of Household Composition Effects and the Cost of Children", Economic Journal, Vol. 87, S. 460 - 487.

Nebenführ, Eva (1995), „Determinanten für die Geburt eines zweiten Kindes”, Zeitschrift für Bevölkerungswissenschaft, Vol. 20, S. 207 - 214.

Nell-Breuning, Oswald v. (1981), „Drei Generationen in Solidarität - Rückbesinnung auf den echten Schreiber-Plan", in: Oswald von Nell-Breuning, Cornelius G. Fetsch (Hrsg.), Drei Generationen in Solidarität, Beiträge zur Gesellschaftspolitik 18, Köln, S. 27 - 42.

Nerlove, Marc, Razin, Assaf, Sadka, Efraim (1984), „Bequests and the Size of Population When Population is Endogenous", Journal of Political Economy, Vol. 92, S. 527 - 531.

Nerlove, Marc, Razin, Assaf, Sadka, Efraim (1985), „Population Size: Individual Choice and Social Optima", Quarterly Journal of Economics, Vol. 100, S. 321 334.

Nerlove, Marc, Razin, Assaf, Sadka, Efraim (1987), Household and economy, Boston u.a.O.

Neumann, Manfred (1986), „Entlastung der Gesetzlichen Rentenversicherung durch kapitalbildende Maßnahmen", in: Bernhard Felderer (Hrsg.), Kapitaldeckungsverfahren versus Umlageverfahren, Schriften des Vereins für Socialpolitik, Neue Folge, Band 163, Berlin, 1986, S. 27 - 53.

Nipperdey, Thomas (1990), Deutsche Geschichte 1866 - 1918, Erster Band: Arbeitswelt und Bürgergeist, München.

Oeter, Ferdinand (1989a), „Familienlastenausgleich und Generationenvertrag”, Sozialer Fortschritt, Vol. 38, S. 241 - 244.

Oeter, Ferdinand (1989b), „Ausverkauf der Familie in der Bundesrepublik Deutschland?", Zeitschrift für Sozialreform, Vol. 35, S. 44 - 52. 
Oeter, Ferdinand (1997), „Die vier Säulen sozialer Gerechtigkeit und Sicherheit”, Zeitschrift für Sozialreform, Vol. 43, S. 397 - 406.

Perozek, Maria G. (1998), „A Reexamination of the Strategic Bequest Motive”, Journal of Political Economy, Vol. 106, S. 423 - 445.

Peters, Wolfgang (1995), „Public Pensions, family allowances and endogenous demographic change", Journal of Pupulation Economics, Vol. 8, S. 161 - 183.

Petersen, Jorn Henrik (1984), „The Political Economy of Financing Old-Age Pensions", Zeitschrift für die gesamte Staatswissenschaft, Vol. 140, S. 430 - 447.

Philipson, Thomas J., Becker, Gary S. (1998), „Old-Age Longevity and MortalityContingent Claims", Journal of Political Economy, Vol. 106, S. 551 - 573.

Pohl, Katharina (1975), „Ergebnisse der Longitudinaluntersuchung Generatives Verhalten in der Bundesrepublik Deutschland", Zeitschrift für Bevölkerungswissenschaft, Vol. 1, S. 53 - 65.

Pohl, Katharina (1985), „Wende - oder Einstellungswandel?”, Zeitschrift für Bevölkerungswissenschaft, Vol. 11, S. 89 - 110.

Pohl, Katharina (1995), „Kinderwunsch und Familienplanung in Ost- und Westdeutschland", Zeitschrift für Bevölkerungswissenschaft, Vol. 20 (1995), S. 67 100 .

Pollak, Robert A. (1985), „A Transaction Cost Approach to Families and Households", Journal of Economic Literature, Vol. 23, S. 581 - 608.

Prinz, Aloys, Klanberg, Frank (1985), „Vexierbilder der neuen Familienpolitik”, Wirtschaftsdienst, Vol. 65, S. 456 - 463.

Proebsting, Helmut (1983), „Kinderzahl ausgewählter Bevölkerungsgruppen”, Wirtschaft und Statistik, Heft 11 / 1983, S. 858 - 868.

Raffelhüschen, Bernd (1998), Interne Renditen gemäß der Generationenbilanz, in: Deutsches Institut für Altersvorsorge (Hrsg.), Renditen der gesetzlichen Rentenversicherung im Vergleich zu alternativen Anlageformen, Frankfurt / Main, S. 36 -49 . 
Robins, Philip K., Spiegelman, Robert G. (1978), „An Econometric Model of the Demand for Child Care", Economic Inquiry, Vol. 16, S. 83 - 94.

Rosati, Furio C. (1996), „Social Security in a non-altruistic model with uncertainty and endogenous fertility", Journal of Public Economics, Vol. 60, S. 283 294.

Rosen, Sherwin (1996), „Public Employment and the Welfare State in Sweden”, Journal of Economic Literature, Vol. 34, S. 729 - 740.

Rosenstiel, Lutz v. (1977), "Zur Motivation des generativen Verhaltens - theoretische Konzepte und Untersuchungsansätze", in: Bundesinstitut für Bevölkerungsforschung (Hrsg.), Materialien zur Bevölkerungswissenschaft, Heft 9, Ursachen des Bevölkerungsrückgangs und seine Konsequenzen für die deutsche Wirtschaft, Tagung 7. - 10.12.1977, ohne Ort, ohne Jahr, S. 91 - 104.

Rothschild, Michael, Stiglitz, Joseph E. (1976), „Equilibrium in competitive insurance markets: an essay in the economics of incomplete information", Quarterly Journal of Economics, Vol. 90, S. 624 - 649.

Rückert, Gerd-Rüdiger (1976), „Die Kinderzahl der Ehen in der Bundesrepublik Deutschland im Intergenerationenvergleich", Zeitschrift für Bevölkerungswissenschaft, Vol. 2, S. 36 - 52.

Samuelson, Paul A. (1958), „An Exact Consumption-Loan Model of Interest with or without the Social Contrivance of Money", Journal of Political Economy, Vol. 66, S. 467 - 482.

Schmähl, Winfried (1997), „Alterssicherung - Quo Vadis?”, Jahrbücher für Nationalökonomie und Statistik, Vol. 216, S. 413 - 435.

Schlenger, Michael (1998), Versicherungsfremde Leistungen in der Gesetzlichen Rentenversicherung, Karl-Bräuer-Institut des Bundes der Steuerzahler e.V., Wiesbaden.

Schmid, Josef (1988), „On contemporary demographic structures in Europe: The explanatory power of economic and sociological frameworks", Zeitschrift für Bevölkerungswissenschaft, Vol. 14, S. 115 - 132. 
Schmidt, Henning, Frank, Udo, Müller-Rohr, Iris (1985), „Kritische Bemerkungen zum System des Kinderlastenausgleichs - zugleich ein Vorschlag zur Neugestaltung der gesetzlichen Rentenversicherung", Finanzarchiv, Vol. 43, S. 28 66.

Schmidt, Michael, Gasch, Thomas (1993), „Rentenversicherung im Spagat zwischen Familien- und Arbeitsmarktpolitik", Sozialer Fortschritt, Vol. 42, S. 268 276.

Schmidt-Kaler, Thomas (1978), „Rentengesetzgebung als Instrument zur rationalen Steuerung und Rückkopplung des Bevölkerungsprozesses", Zeitschrift für Bevölkerungswissenschaft, Vol. 4, S. 75 - 88.

Schnabel, Reinhold (1998a), Rates of Return of the German Pay-As-You-Go Pension System, Working Paper, Sonderforschungsbereich 504 an der Universität Mannheim, Mannheim.

Schnabel, Reinhold (1998b), Interne Renditen in der gesetzlichen Rentenversicherung, in: Deutsches Institut für Altersvorsorge (Hrsg.), Renditen der gesetzlichen Rentenversicherung im Vergleich zu alternativen Anlageformen, Frankfurt / Main, S. 15 - 35.

Schneewind, Klaus A. (1977), „Eltern-Kind-Beziehungen als Determinanten des generativen Verhaltens", in: Bundesinstitut für Bevölkerungsforschung (Hrsg.), Materialien zur Bevölkerungswissenschaft, Heft 9, Ursachen des Bevölkerungsrückgangs und seine Konsequenzen für die deutsche Wirtschaft, Tagung 7. 10.12.1977, ohne Ort, ohne Jahr, S. 119 - 132.

Schreiber, Wilfried (1955), Existenzsicherheit in der industriellen Gesellschaft, wiederabgedruckt (in Auszügen) in: Oswald von Nell-Breuning, Cornelius G. Fetsch (Hrsg.), Drei Generationen in Solidarität, Beiträge zur Gesellschaftspolitik 18, Köln, 1981, S. $81-100$.

Schwarz, Karl (1977), „Umfang und demographische Konsequenzen des Geburtenrückgangs in der Bundesrepublik Deutschland", in: Bundesinstitut für Bevölkerungsforschung (Hrsg.), Materialien zur Bevölkerungswissenschaft, Heft 9, Ursachen des Bevölkerungsrückgangs und seine Konsequenzen für die deutsche Wirtschaft, Tagung 7. - 10.12.1977, ohne Ort, ohne Jahr, S. 7 - 18. 
Schwarz, Karl (1982), „Kinderzahl der Ehen nach Bevölkerungsgruppen im Jahre 1981", Zeitschrift für Bevölkerungswissenschaft, Vol. 8, S. 575 - 587.

Schwarz, Karl (1985), „Umfang der Frauenerwerbstätigkeit nach dem Zweiten Weltkrieg", Zeitschrift für Bevölkerungswissenschaft, Vol. 11, S. 241 - 260.

Schwarz, Karl (1988), „Demographische und ökonomische Überlegungen zur kinderlosen Ehe", Zeitschrift für Bevölkerungswissenschaft, Vol. 14, S. 101 - 103.

Schwarz, Karl (1990a), „Die Bildungsabschlüsse der Frauen und ihre Bedeutung für den Arbeitsmarkt, die Eheschließung und die Familienbildung", Zeitschrift für Bevölkerungswissenschaft, Vol. 16, S. 361 - 382.

Schwarz, Karl (1990b), „Wieviele Kinder haben die Familien?”, Zeitschrift für Bevölkerungswissenschaft, Vol. 16, S. 435 - 445.

Schwarz, Karl (1991), „Kinderzahl der Frauen der Geburtsjahrgänge 1865 1955”, Zeitschrift für Bevölkerungswissenschaft, Vol. 17, S. 149 - 157.

Schwarz, Karl (1992a), „Differenzielle Kinderzahlen in Westdeutschland im Jahr 1990", Zeitschrift für Bevölkerungswissenschaft, Vol. 18, S. 143 - 147.

Schwarz, Karl (1992b), „Bevölkerungspolitische Wirkungen familienpolitischer Maßnahmen", Zeitschrift für Bevölkerungswissenschaft, Vol. 18, S. 197 - 208.

Schwarz, Karl (1993a), „Kinder und Jugendliche in den Familien Alleinstehender in Deutschland im Jahr 1991", Zeitschrift für Bevölkerungswissenschaft, Vol. 19 (1993 / 94), S. 71 - 91.

Schwarz, Karl (1993b), „Frauenerwerbstätigkeit im Lebenslauf gestern und heute", Zeitschrift für Bevölkerungswissenschaft, Vol. 19 (1993/94), S. 541 - 575.

Schwarz, Karl (1984), „Eltern und Kinder in unvollständigen Familien”, in: Zeitschrift für Bevölkerungswissenschaft, Vol. 10, S. 3 - 36.

Schwarz, Karl (1995), „In welchen Familien wachsen die Kinder und Jugendlichen in Deutschland auf?”, Zeitschrift für Bevölkerungswissenschaft, Vol. 20, S. $271-292$. 
Schwarz, Karl (1998), „Überlegungen zum Erreichen einer quasi stationären Bevölkerung”, Zeitschrift für Bevölkerungswissenschaft, Vol. 23, S. 211 - 214.

Seneca, Joseph, Taussig, Michael K. (1971), „Family Equivalence Scales and Personal Income Tax Exemptions for Children", Review of Economics and Statistics, Vol. 53, S. $253-262$.

Sinn, Hans-Werner (1997), „The Value of Children and Immigrants in an Pay-asyou-go Pension System: A Proposal for a Partial Transition to a Funded System", CES Working Paper Series, WP No. 141, München.

Sinn, Hans-Werner (1998), „The Pay-as-you-go Pension System as a Fertility Insurance and Enforcement Device", CES Working Paper Series, WP No. 154, München.

Sinn, Hans-Werner, Thum, Michael (1999), „Gesetzliche Rentenversicherung: Prognosen im Vergleich", CES-ifo Paper, München.

Smith, Alasdair (1982), „Intergenerational Transfers as Social Insurance”, Journal of Public Economics, Vol. 19, S. 97 - 106.

Spieß, Erika et al. (1984), „Wertwandel und generatives Verhalten”, Zeitschrift für Bevölkerungswissenschaft, Vol. 10, S. 153 - 168.

Spremann, Klaus (1984), „Intergenerational Contracts and Their Decomposition”, Zeitschrift für Nationalökonomie / Journal of Economics, Vol. 44, S. 237 - 253.

Spremann, Klaus (1986), „Generationenverträge bei insttionärer Wirtschaftsentwicklung", in: Bernhard Felderer (Hrsg.), Kapitaldeckungsverfahren versus Umlageverfahren, Schriften des Vereins für Socialpolitik, Neue Folge, Band 163, Berlin, S. 15 - 25.

Statistisches Bundesamt (Hrsg.) (1995), Im Blickpunkt: Familien heute, MetzlerPoeschel, Stuttgart, 1995.

Störtzbach, Bernd (1993), „Deutschland nach der Vereinigung - Meinungen und Einstellungen zu Familie, Kindern und zur Familienpolitik in Ost und West", Zeitschrift für Bevölkerungswissenschaft, Vol. 19 (1993/94), S. 151 - 187. 
Strawczynski, Michel (1994), „Government intervention as a bequest substitute”, Journal of Public Economics, Vol. 53, S. 477 - 495.

Suhr, Dieter (1990), „Transferrechtliche Ausbeutung und verfassungsrechtlicher Schutz von Familien, Müttern und Kindern", Der Staat, Vol. 29, S. 69 - 87.

Urdze, Andrejs (1977), „Bestimmungsfaktoren eingeschränkten Kinderwunsches untersucht an Ein-Kind-Familien in Bayern", in: Bundesinstitut für Bevölkerungsforschung (Hrsg.), Materialien zur Bevölkerungswissenschaft, Heft 9, Ursachen des Bevölkerungsrückgangs und seine Konsequenzen für die deutsche Wirtschaft, Tagung 7. - 10.12.1977, ohne Ort, ohne Jahr, S. 151 - 161.

Verband Deutscher Rentenversicherungsträger (Hrsg.) (1997), Versicherungsfremde Leistungen - sachgerecht finanzieren!, Reihe: Fakten und Argumente, Heft Nr. 5, Frankfurt / Main.

Wagner, Gert (1984), Umverteilung in der Gesetzlichen Rentenversicherung, Frankfurt / Main.

Weber, Axel (1987), „Zur These künftiger ,Unfinanzierbarkeit' der Sozialversicherung", Sozialer Fortschritt, Vol. 36, S. 49 - 53.

Weil, David N. (1999), „Population Growth, Dependency, and Consumption“, American Economic Review, Papers and Proceedings, Vol. 89, S. 251 - 255.

Werding, Martin (1998), Zur Rekonstruktion des Generationenvertrages, Beiträge zur Finanzwissenschaft 3, Tübingen.

Wilhelm, Mark O. (1996), „Bequest Behavior and the Effect of Heirs' Earnings: Testing the Altruistic Model of Bequests", American Economic Review, Vol. 86, S. $874-892$.

Willis, Robert J. (1974), „Economic Theory of Fertility Behavior“, in: Theodore W. Schultz (Hrsg.), Economics of the Family - Marriage, Children, and Human Capital, Chicago, London, 1974, S. 25 - 80; abgedruckt (unter dem Titel „A New Approach to the Economic Theory of Fertility Behavior") auch in: Journal of Political Economy, Vol. 81 (1973), S. S14 - S64. 
Winegarden, C.R. (1984), „Women's Fertility, Market Work and Marital Status: A Test of the New Household Economics with International Data", Economica, Vol. 51, S. 447 - 456.

Wissenschaftlicher Beirat beim Bundesministerium der Finanzen (1974), Entschließungen, Stellungnahmen und Gutachten 1949 - 1973, (Hrsg. vom Bundesministerium der Finanzen), Tübingen.

Wissenschaftlicher Beirat beim Bundesministerium für Wirtschaft (1998), Grundlegende Reform der gesetzlichen Rentenversicherung, Gutachten, Bonn.

Zimmermann, Klaus F. (1982), „Humankapital, Kinderwunsch und Familiengröße", Zeitschrift für Bevölkerungswissenschaft, Vol. 8, S. 547 - 558.

Zimmermann, Klaus F. (1984), „Grenzen einer Bevölkerungspolitik durch Familienpolitik", Wirtschaftsdienst, Vol. 64, S. 180 - 185.

Zimmermann, Klaus F. (1985), Familienökonomie, Berlin u.a.O.

Entscheidungen des Bundesverfassungsgerichts

- vom 07.07.1992 (87, 1, I, Nr. 1)

- vom 12.03.1996 $(94,241$, I, Nr. 6$)$

\section{Zeitungen, Zeitschriften und Periodika}

Bundesrats-Drucksache 552 / ‘97 vom 24.07.1997.

Deutscher Bundestag Drucksache 14 / 45 vom 17.11.1998.

Bundesverfassungsgericht, Entscheidungen (BVerfGE), div. Ausgaben, div. Jahrgänge (Anmerkung: Offizielle Herausgeber sind die jeweiligen Richter am Bundesverfassungsgericht.)

Wirtschaftswoche Nr. 31 / 1998 vom 23.07.1998. 


\section{Gesetzestexte}

Einkommensteuergesetz (EStG)

Mutterschutzgesetz (MuSchG)

Sozialgesetzbuch VI (SGB VI)

\section{Statistische Werke}

Statistisches Bundesamt (Hrsg.), Statistisches Jahrbuch für Deutschland, div. Jahrgänge, Stuttgart.

Statistisches Bundesamt (Hrsg.), Fachserie 1 (Bevölkerung und Erwerbstätigkeit) Reihe 1 (Gebiet und Bevölkerung), Stuttgart, div. Jgg.

Statistisches Bundesamt (Hrsg.), Fachserie 1 (Bevölkerung und Erwerbstätigkeit) Reihe 4.1.1 (Stand und Entwicklung der Erwerbstätigkeit), Stuttgart, div. Jgg.

Verband Deutscher Rentenversicherungsträger (Hrsg.) (1999), Rentenversicherung in Zeitreihen, Ausgabe 1999, Frankfurt / Main. 


\section{SCHRIFTEN ZUR WIRTSCHAFTSTHEORIE UND WIRTSCHAFTSPOLITIK}

Herausgegeben von Rolf Hasse, Jörn Kruse, Wolf Schäfer, Thomas Straubhaar, Klaus W. Zimmermann

Band 1 Lars Būnning: Die Konvergenzkriterien des Maastricht-Vertrages unter besonderer Berücksichtigung ihrer Konsistenz. 1997.

Band 2 Andreas Henning: Beveridge-Kurve, Lohnsetzung und Langzeitarbeitslosigkeit. Eine theoretische Untersuchung unter Berücksichtigung des Insider-Outsider-Ansatzes und der Entwertung des Humankapitals. 1997.

Band 3 Iris Henning: Die Reputation einer Zentralbank. Eine theoretische Untersuchung unter besonderer Berücksichtigung der Europäischen Zentralbank. 1997.

Band 4 Rüdiger Hermann: Ein gemeinsamer Markt für Elektrizität in Europa. Optionen einer Wettbewerbsordnung zwischen Anspruch und Wirklichkeit. 1997.

Band 5 Alexander Tiedtke: Japan und der Vorwurf des Trittbrettfahrerverhaltens in der US-amerikanisch-japanischen Allianz. 1997.

Band 6 Wolfgang Grimme: Ordnungspolitisches Konzept der Regionalpolitik. Darstellung der Defizite und des Reformbedarfs der Regionalpolitik am Beispiel Meckenburg-Vorpommems. 1997.

Band 7 Christian Ricken: Determinanten der Effektivität der Umweltpolitik. Der nationale Politikstil im Spannungsfeld von Ōkonomie, Politik und Kultur. 1997.

Band 8 Christian Schmidt: Real Convergence in the European Union. An Empirical Analysis. 1997.

Band 9 Silvia Marengo: Exchange Rate Policy for MERCOSUR: Lessons from the European Union. 1998.

Band 10 Jens Kleinemeyer: Standardisierung zwischen Kooperation und Wettbewerb. Eine spieltheoretische Betrachtung. 1998.

Band 11 Stefan M. Golder: Migration und Arbeitsmarkt. Eine empirische Analyse der Performance von Ausländern in der Schweiz. 1999.

Band 12 Stefan Kramer: Die Wirkung einer Internationalisierung des Yen auf die japanischen Finanzmärkte, die japanische Geldpolitik und die Usancen der Fakturierung. 1999.

Band 13 Antje Marielle Gerhold: Wirtschaftliche Integration und Kooperation im asiatisch-pazifischen Raum. Die APEC. 1999.

Band 14 Tamim Achim Dawar: Deutsche Direktinvestitionen in Australien. Eine Evaluation der theoretischen Erklärungsansätze und der Standortattraktivităt des Produktions- und Investitionsstandortes Australien. 1999.

Band 15 Hans-Markus Johannsen: Die ordnungspolitische Haltung Frankreichs im Prozeß der europäischen Einigung. 1999.

Band 16 Annette Schönherr. Vereinigungsbedingte Dimensionen regionaler Arbeitsmobilität. Wirkungen unter analytisch einfachen Bedingungen und potentielle individuelle Migrationsgewinne in Deutschland nach der Vereinigung. 1999.

Band 17 Henrik Müller. Wechselkurspolitik des Eurolandes. Konfliktstoff für die neue währungspolitische Ära. 1999.

Band 18 Lars H. Wengorz: Die Bedeutung von Unternehmertum und Eigentum für die Existenz von Unternehmen. Eine methodenkritische Analyse der Transformation des Wirtschaftssystems in Russland. 2000.

Band 19 Eckart Bauer. Konzeptionelle Grundfragen eines Kinderleistungsausgleichs im Rahmen einer umlagefinanzierten zwangsweisen Rentenversicherung. 2000. 
Eckart Bauer - 978-3-631-74995-1

Downloaded from PubFactory at 01/11/2019 09:28:58AM

via free access 


\section{Zwischen Arbeitslosigkeit und Überstunden}

\section{Personalwirtschaftliche Überlegungen zur Verteilung von Arbeitsvolumina}

Frankfurt/M., Berlin, Bern, Bruxelles, New York, Wien, 2000. $231 \mathrm{~S}$. ISBN 3-631-35685-4 • br. DM 58.-*

Die Autorinnen des Sammelbandes untersuchen die Verteilung von Arbeitsvolumina aus personalwirtschaftlicher Perspektive. Warum werden Überstunden geleistet, obwohl Arbeitslosigkeit herrscht? Wann werden Arbeitsvolumina unter Vertragsformen wie Telearbeit, Zeitarbeit oder geringfügiger Beschäftigung abgeleistet? Zur Beantwortung dieser Fragen werden empirische Befunde mit theoretischen Überlegungen zu den personalwirtschaftlichen Einflußfaktoren auf die Verteilung von Arbeitsvolumina kontrastiert. Insbesondere wird untersucht, ob aktuelle arbeitsrechtliche Maßnahmen zu dem vielfach postulierten Ziel einer breiteren personellen Verteilung von Arbeitsvolumina führen. Ein gemeinsames Ergebnis der Einzelbeiträge ist, daß aufgrund der vielfältigen Mechanismen hinter der Verteilung von Arbeitsvolumina isolierte Maßnahmen kaum zu einem Abbau der Arbeitslosigkeit führen werden.

Aus dem Inhalt: Verteilung von Arbeitsvolumina - Überstundenabbau . Geringfügige Beschäftigung · Telearbeit · Arbeitnehmerüberlassung · Kündigungsschutz in Kleinbetrieben - Normalarbeitsverhältnisse

Frankfurt/M - Berlin - Bern · New York · Paris - Wien

Auslieferung: Verlag Peter Lang AG

Jupiterstr. 15, $\mathrm{CH}-3000$ Bern 15

Telefax (004131) 9402131

*inklusive Mehrwertsteuer

Preisänderungen vorbehalten 
Eckart Bauer - 978-3-631-74995-1

Downloaded from PubFactory at 01/11/2019 09:28:58AM

via free access 